\title{
TOXICOLOGICAL PROFILE FOR
} PHENOL

\author{
U.S. DEPARTMENT OF HEALTH AND HUMAN SERVICES \\ Public Health Service \\ Agency for Toxic Substances and Disease Registry
}

September 2008 


\section{DISCLAIMER}

The use of company or product name(s) is for identification only and does not imply endorsement by the Agency for Toxic Substances and Disease Registry. 


\section{UPDATE STATEMENT}

A Toxicological Profile for Phenol, Draft for Public Comment was released in October 2006. This edition supersedes any previously released draft or final profile.

Toxicological profiles are revised and republished as necessary. For information regarding the update status of previously released profiles, contact ATSDR at:

Agency for Toxic Substances and Disease Registry

Division of Toxicology and Environmental Medicine/Applied Toxicology Branch

1600 Clifton Road NE

Mailstop F-32

Atlanta, Georgia 30333 
This page is intentionally blank. 


\section{FOREWORD}

This toxicological profile is prepared in accordance with guidelines developed by the Agency for Toxic Substances and Disease Registry (ATSDR) and the Environmental Protection Agency (EPA). The original guidelines were published in the Federal Register on April 17, 1987. Each profile will be revised and republished as necessary.

The ATSDR toxicological profile succinctly characterizes the toxicologic and adverse health effects information for the hazardous substance described therein. Each peer-reviewed profile identifies and reviews the key literature that describes a hazardous substance's toxicologic properties. Other pertinent literature is also presented, but is described in less detail than the key studies. The profile is not intended to be an exhaustive document; however, more comprehensive sources of specialty information are referenced.

The focus of the profiles is on health and toxicologic information; therefore, each toxicological profile begins with a public health statement that describes, in nontechnical language, a substance's relevant toxicological properties. Following the public health statement is information concerning levels of significant human exposure and, where known, significant health effects. The adequacy of information to determine a substance's health effects is described in a health effects summary. Data needs that are of significance to protection of public health are identified by ATSDR and EPA.

Each profile includes the following:

(A) The examination, summary, and interpretation of available toxicologic information and epidemiologic evaluations on a hazardous substance to ascertain the levels of significant human exposure for the substance and the associated acute, subacute, and chronic health effects;

(B) A determination of whether adequate information on the health effects of each substance is available or in the process of development to determine levels of exposure that present a significant risk to human health of acute, subacute, and chronic health effects; and

(C) Where appropriate, identification of toxicologic testing needed to identify the types or levels of exposure that may present significant risk of adverse health effects in humans.

The principal audiences for the toxicological profiles are health professionals at the Federal, State, and local levels; interested private sector organizations and groups; and members of the public.

This profile reflects ATSDR's assessment of all relevant toxicologic testing and information that has been peer-reviewed. Staff of the Centers for Disease Control and Prevention and other Federal scientists have also reviewed the profile. In addition, this profile has been peer-reviewed by a nongovernmental panel 
and was made available for public review. Final responsibility for the contents and views expressed in this toxicological profile resides with ATSDR.

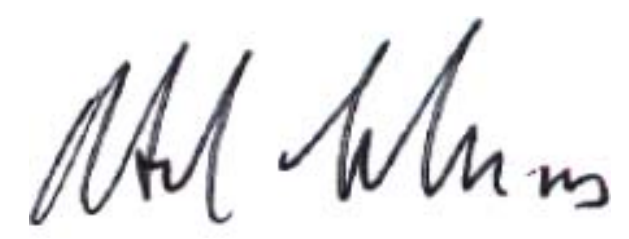

Howard Frumkin M.D., Dr.P.H.

Director

National Center for Environmental Health/

Agency for Toxic Substances and

Disease Registry

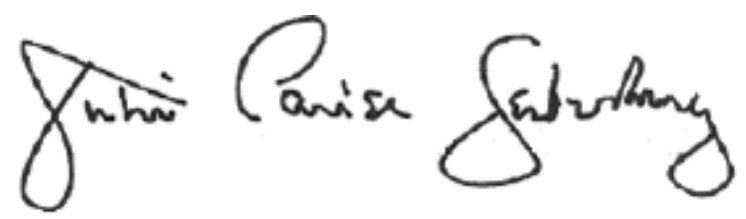

Julie Louise Gerberding, M.D., M.P.H.

Administrator

Agency for Toxic Substances and

Disease Registry

\section{* Legislative Background}

The toxicological profiles are developed in response to the Superfund Amendments and Reauthorization Act (SARA) of 1986 (Public Law 99 499) which amended the Comprehensive Environmental Response, Compensation, and Liability Act of 1980 (CERCLA or Superfund). This public law directed ATSDR to prepare toxicological profiles for hazardous substances most commonly found at facilities on the CERCLA National Priorities List and that pose the most significant potential threat to human health, as determined by ATSDR and the EPA. The availability of the revised priority list of 275 hazardous substances was announced in the Federal Register on December 7, 2005 (70 FR 72840). For prior versions of the list of substances, see Federal Register notices dated April 17, 1987 (52 FR 12866); October 20, 1988 (53 FR 41280); October 26, 1989 (54 FR 43619); October 17,1990 (55 FR 42067); October 17, 1991 (56 FR 52166); October 28, 1992 (57 FR 48801); February 28, 1994 (59 FR 9486); April 29, 1996 (61 FR 18744); November 17, 1997 (62 FR 61332); October 21, 1999(64 FR 56792); October 25, 2001 (66 FR 54014) and November 7, 2003 (68 FR 63098). Section 104(i)(3) of CERCLA, as amended, directs the Administrator of ATSDR to prepare a toxicological profile for each substance on the list. 


\section{QUICK REFERENCE FOR HEALTH CARE PROVIDERS}

Toxicological Profiles are a unique compilation of toxicological information on a given hazardous substance. Each profile reflects a comprehensive and extensive evaluation, summary, and interpretation of available toxicologic and epidemiologic information on a substance. Health care providers treating patients potentially exposed to hazardous substances will find the following information helpful for fast answers to often-asked questions.

\section{Primary Chapters/Sections of Interest}

Chapter 1: Public Health Statement: The Public Health Statement can be a useful tool for educating patients about possible exposure to a hazardous substance. It explains a substance's relevant toxicologic properties in a nontechnical, question-and-answer format, and it includes a review of the general health effects observed following exposure.

Chapter 2: Relevance to Public Health: The Relevance to Public Health Section evaluates, interprets, and assesses the significance of toxicity data to human health.

Chapter 3: Health Effects: Specific health effects of a given hazardous compound are reported by type of health effect (death, systemic, immunologic, reproductive), by route of exposure, and by length of exposure (acute, intermediate, and chronic). In addition, both human and animal studies are reported in this section.

NOTE: Not all health effects reported in this section are necessarily observed in the clinical setting. Please refer to the Public Health Statement to identify general health effects observed following exposure.

Pediatrics: Four new sections have been added to each Toxicological Profile to address child health issues:
Section 1.6
How Can (Chemical X) Affect Children?
Section 1.7
Section 3.7
How Can Families Reduce the Risk of Exposure to (Chemical X)?
Section 6.6
Children's Susceptibility
Exposures of Children

Other Sections of Interest:

Section $3.8 \quad$ Biomarkers of Exposure and Effect

Section 3.11 Methods for Reducing Toxic Effects

\section{ATSDR Information Center \\ Phone: 1-800-CDC-INFO (800-232-4636) or 1-888-232-6348 (TTY) Fax: (770) 488-4178 \\ E-mail: cdcinfo@cdc.gov Internet: http://www.atsdr.cdc.gov}

The following additional material can be ordered through the ATSDR Information Center:

Case Studies in Environmental Medicine: Taking an Exposure History-The importance of taking an exposure history and how to conduct one are described, and an example of a thorough exposure history is provided. Other case studies of interest include Reproductive and Developmental Hazards; Skin Lesions and Environmental Exposures; Cholinesterase-Inhibiting Pesticide Toxicity; and numerous chemical-specific case studies. 
Managing Hazardous Materials Incidents is a three-volume set of recommendations for on-scene (prehospital) and hospital medical management of patients exposed during a hazardous materials incident. Volumes I and II are planning guides to assist first responders and hospital emergency department personnel in planning for incidents that involve hazardous materials. Volume IIIMedical Management Guidelines for Acute Chemical Exposures - is a guide for health care professionals treating patients exposed to hazardous materials.

Fact Sheets (ToxFAQs) provide answers to frequently asked questions about toxic substances.

\section{Other Agencies and Organizations}

The National Center for Environmental Health (NCEH) focuses on preventing or controlling disease, injury, and disability related to the interactions between people and their environment outside the workplace. Contact: NCEH, Mailstop F-29, 4770 Buford Highway, NE, Atlanta, GA 30341-3724 • Phone: 770-488-7000 • FAX: 770-488-7015.

The National Institute for Occupational Safety and Health (NIOSH) conducts research on occupational diseases and injuries, responds to requests for assistance by investigating problems of health and safety in the workplace, recommends standards to the Occupational Safety and Health Administration (OSHA) and the Mine Safety and Health Administration (MSHA), and trains professionals in occupational safety and health. Contact: NIOSH, 200 Independence Avenue, SW, Washington, DC 20201 • Phone: 800-356-4674 or NIOSH Technical Information Branch, Robert A. Taft Laboratory, Mailstop C-19, 4676 Columbia Parkway, Cincinnati, OH 45226-1998 - Phone: 800-35-NIOSH.

The National Institute of Environmental Health Sciences (NIEHS) is the principal federal agency for biomedical research on the effects of chemical, physical, and biologic environmental agents on human health and well-being. Contact: NIEHS, PO Box 12233, 104 T.W. Alexander Drive, Research Triangle Park, NC 27709 • Phone: 919-541-3212.

\section{Referrals}

The Association of Occupational and Environmental Clinics (AOEC) has developed a network of clinics in the United States to provide expertise in occupational and environmental issues. Contact: AOEC, 1010 Vermont Avenue, NW, \#513, Washington, DC 20005 • Phone: 202-347-4976 •FAX: 202-347-4950•e-mail: AOEC@AOEC.ORG•Web Page: http://www.aoec.org/.

The American College of Occupational and Environmental Medicine (ACOEM) is an association of physicians and other health care providers specializing in the field of occupational and environmental medicine. Contact: ACOEM, 25 Northwest Point Boulevard, Suite 700, Elk Grove Village, IL 60007-1030 • Phone: 847-818-1800 • FAX: 847-818-9266. 


\section{CONTRIBUTORS}

\section{CHEMICAL MANAGER(S)/AUTHOR(S):}

Jewel Crawford, M.D.

Obaid Faroon, DVM, Ph.D.

Jewell Wilson, Ph.D.

ATSDR, Division of Toxicology and Environmental Medicine, Atlanta, Georgia

Fernando T. Llados, Ph.D.

Kristina Garber, M.S.

Sari J. Paikoff, Ph.D.

Michael H. Lumpkin, Ph.D.

Syracuse Research Corporation, North Syracuse, NY

Syracuse Research Corporation, Arlington, VA

\section{THE PROFILE HAS UNDERGONE THE FOLLOWING ATSDR INTERNAL REVIEWS:}

1. Health Effects Review. The Health Effects Review Committee examines the health effects chapter of each profile for consistency and accuracy in interpreting health effects and classifying end points.

2. Minimal Risk Level Review. The Minimal Risk Level Workgroup considers issues relevant to substance-specific Minimal Risk Levels (MRLs), reviews the health effects database of each profile, and makes recommendations for derivation of MRLs.

3. Data Needs Review. The Applied Toxicology Branch reviews data needs sections to assure consistency across profiles and adherence to instructions in the Guidance.

4. Green Border Review. Green Border review assures the consistency with ATSDR policy. 
This page is intentionally blank. 


\section{PEER REVIEW}

A peer review panel was assembled for phenol. The panel consisted of the following members:

1. Marlissa Campbell, Ph.D., Private Consultant, Pacifica, California;

2. F. Peter Guengerich, Ph.D., Director, Center in Molecular Toxicology, Professor of

Biochemistry, Vanderbilt University, School of Medicine, Nashville, Tennessee; and

3. Thomas Zoeller, Ph.D., Professor, Department of Biology, University of Massachusetts at Amherst, Amherst, Massachusetts;

These experts collectively have knowledge of phenol's physical and chemical properties, toxicokinetics, key health end points, mechanisms of action, human and animal exposure, and quantification of risk to humans. All reviewers were selected in conformity with the conditions for peer review specified in Section 104(I)(13) of the Comprehensive Environmental Response, Compensation, and Liability Act, as amended.

Scientists from the Agency for Toxic Substances and Disease Registry (ATSDR) have reviewed the peer reviewers' comments and determined which comments will be included in the profile. A listing of the peer reviewers' comments not incorporated in the profile, with a brief explanation of the rationale for their exclusion, exists as part of the administrative record for this compound.

The citation of the peer review panel should not be understood to imply its approval of the profile's final content. The responsibility for the content of this profile lies with the ATSDR. 
This page is intentionally blank. 


\section{CONTENTS}

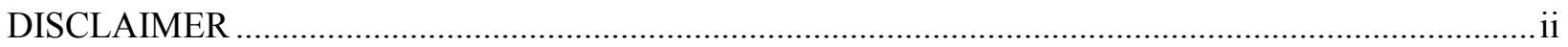

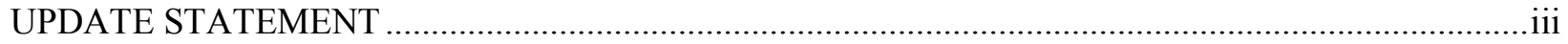

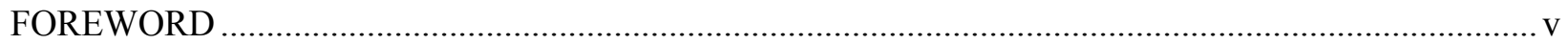

QUICK REFERENCE FOR HEALTH CARE PROVIDERS ...............................................................

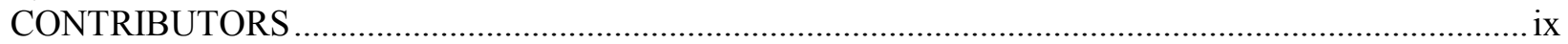

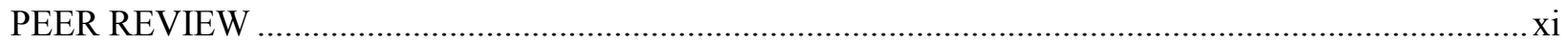

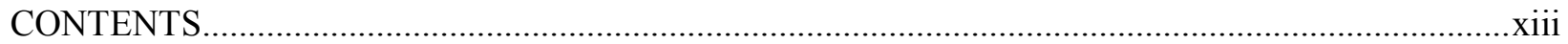

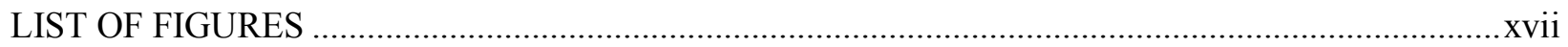

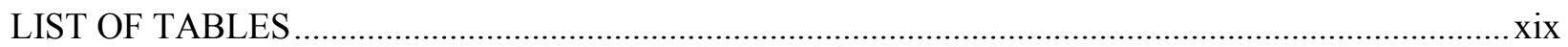

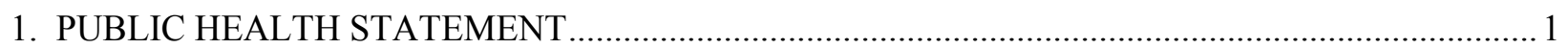

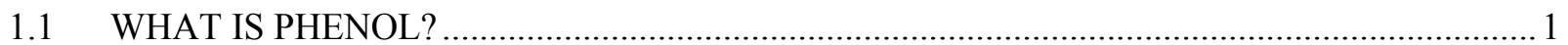

1.2 WHAT HAPPENS TO PHENOL WHEN IT ENTERS THE ENVIRONMENT? .......................... 2

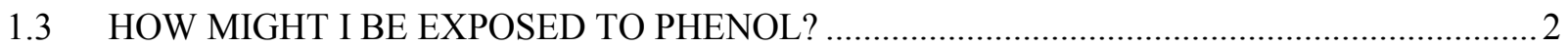

1.4 HOW CAN PHENOL ENTER AND LEAVE MY BODY? ............................................... 3

1.5 HOW CAN PHENOL AFFECT MY HEALTH? ....................................................... 4

1.6 HOW CAN PHENOL AFFECT CHILDREN? ........................................................................ 5

1.7 HOW CAN FAMILIES REDUCE THE RISK OF EXPOSURE TO PHENOL? ....................... 5

1.8 IS THERE A MEDICAL TEST TO DETERMINE WHETHER I HAVE BEEN EXPOSED

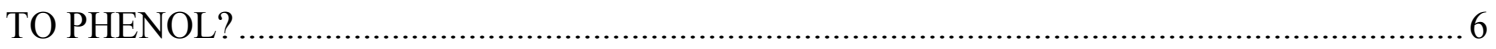

1.9 WHAT RECOMMENDATIONS HAS THE FEDERAL GOVERNMENT MADE TO

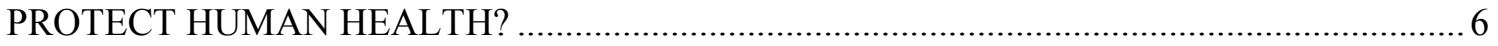

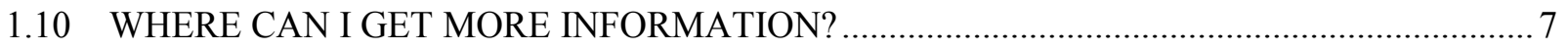

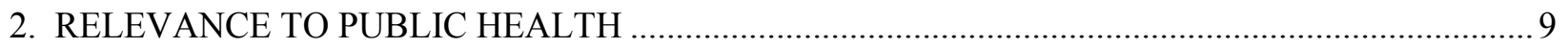

2.1 BACKGROUND AND ENVIRONMENTAL EXPOSURES TO PHENOL IN THE

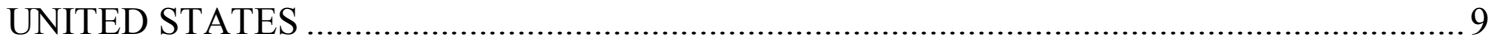

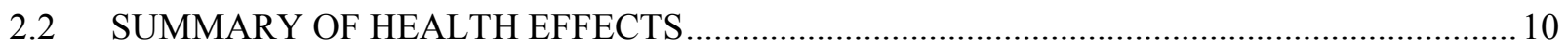

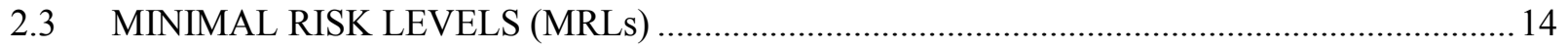

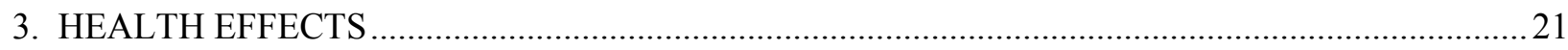

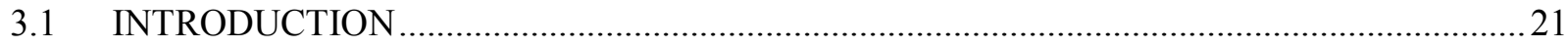

3.2 DISCUSSION OF HEALTH EFFECTS BY ROUTE OF EXPOSURE …............................. 21

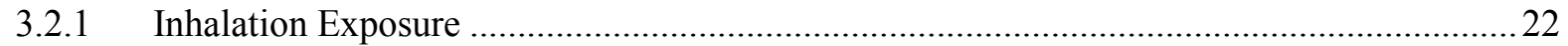

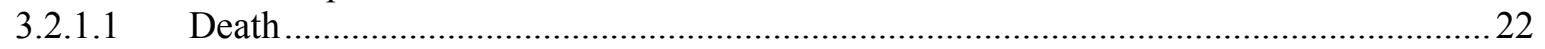

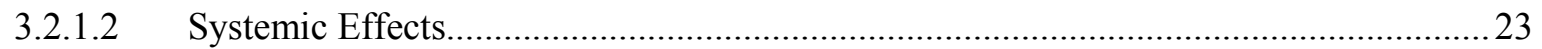

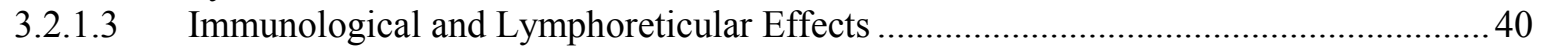

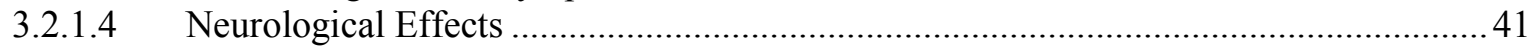

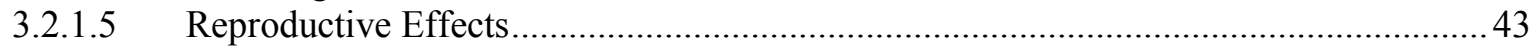

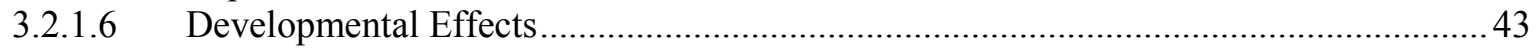

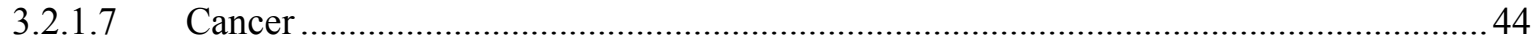

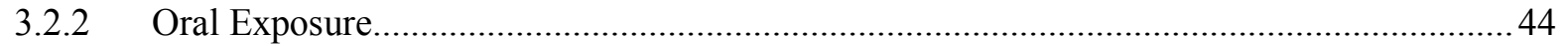

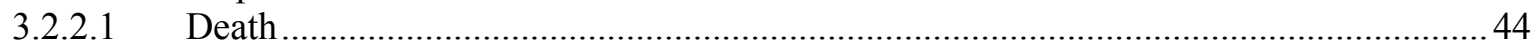

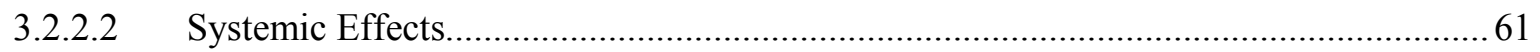

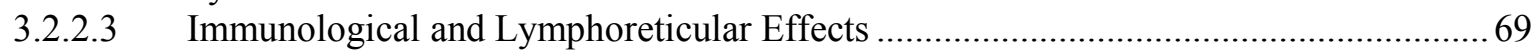

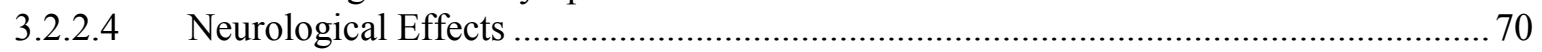

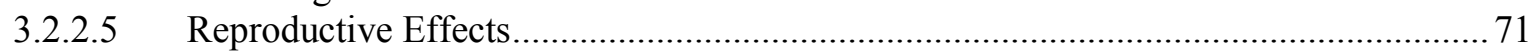

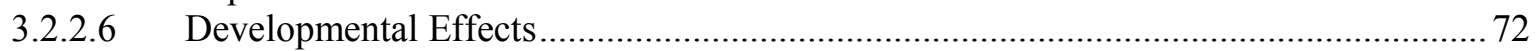

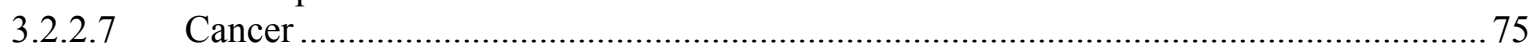




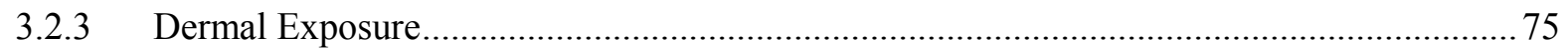

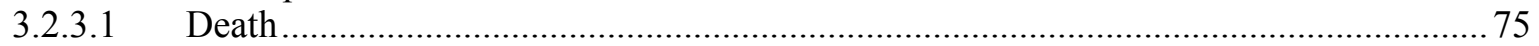

3.2.3.2 Systemic Effects.............................................................................................. 77

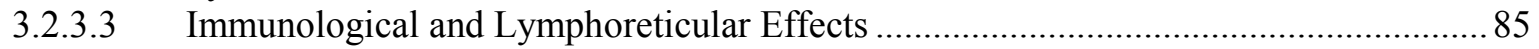

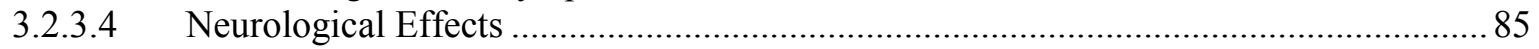

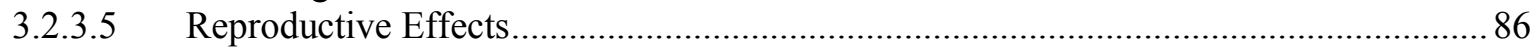

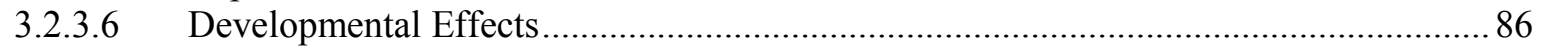

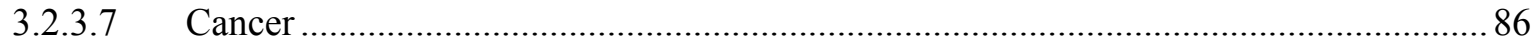

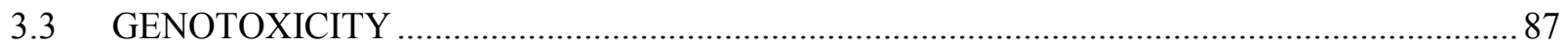

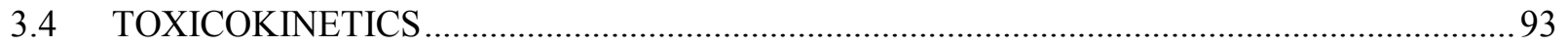

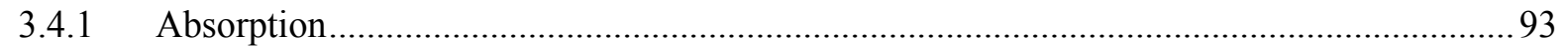

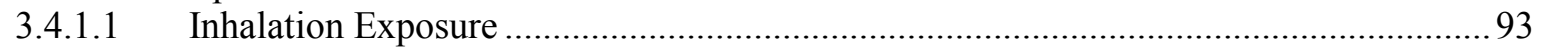

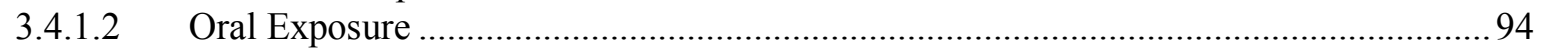

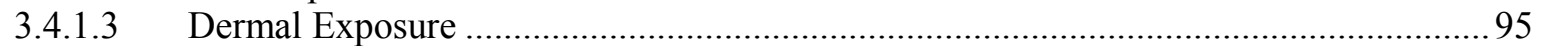

3.4.1.4 Other Routes of Exposure _................................................................................97

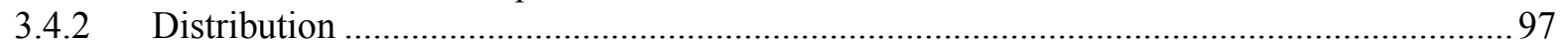

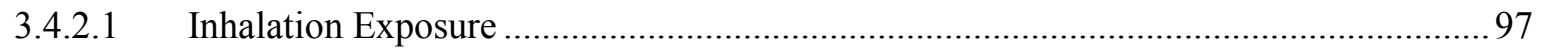

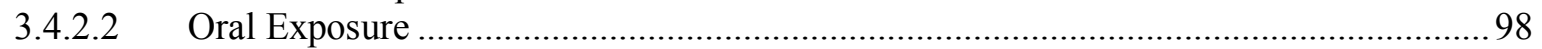

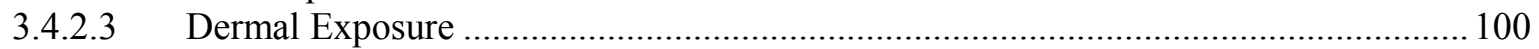

3.4.2.4 Other Routes of Exposure ................................................................................ 100

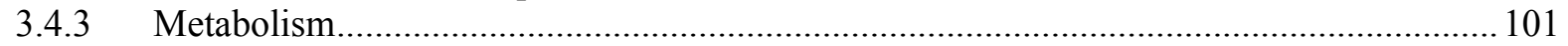

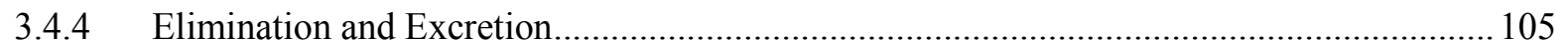

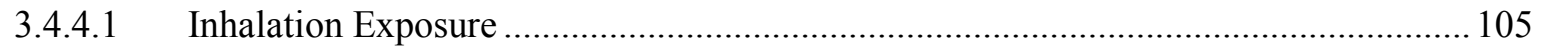

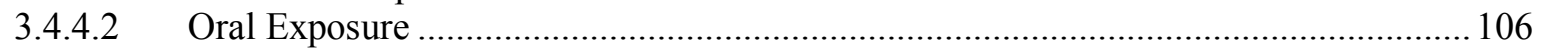

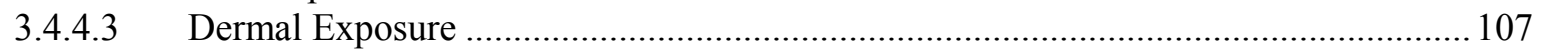

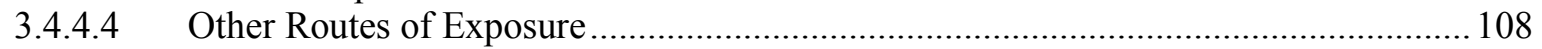

3.4.5 Physiologically Based Pharmacokinetic (PBPK)/Pharmacodynamic (PD) Models ............ 108

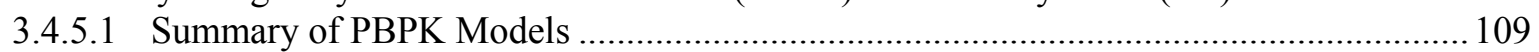

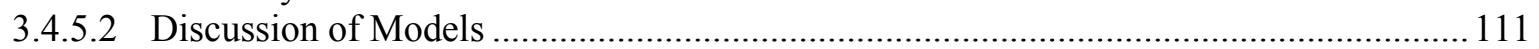

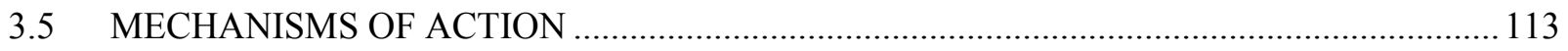

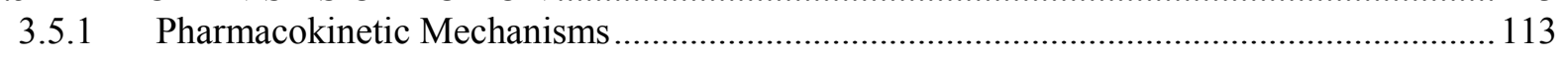

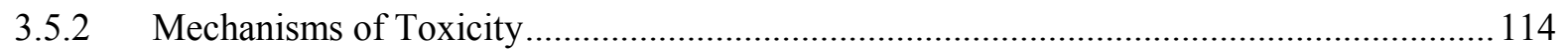

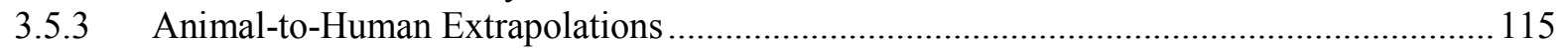

3.6 TOXICITIES MEDIATED THROUGH THE NEUROENDOCRINE AXIS ………………........115

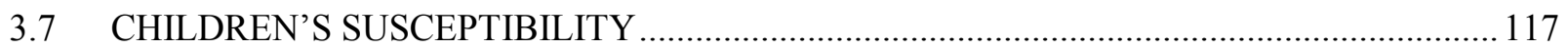

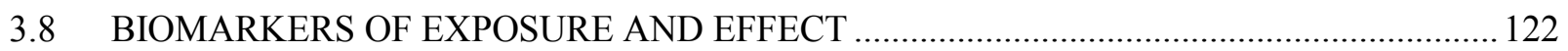

3.8.1 Biomarkers Used to Identify or Quantify Exposure to Phenol ...........................................1 123

3.8.2 Biomarkers Used to Characterize Effects Caused by Phenol............................................... 123

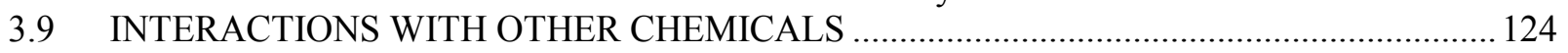

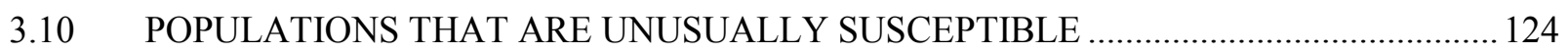

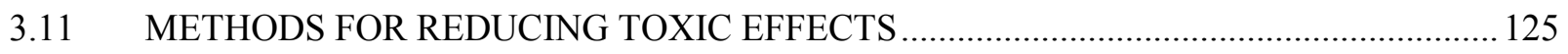

3.11.1 Reducing Peak Absorption Following Exposure.......................................................... 125

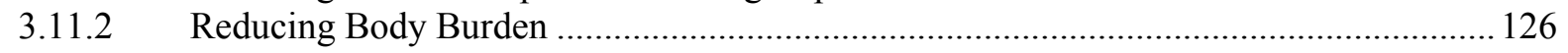

3.11.3 Interfering with the Mechanism of Action for Toxic Effects .......................................... 126

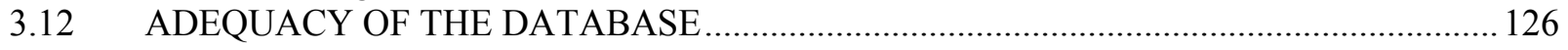

3.12.1 Existing Information on Health Effects of Phenol ......................................................12 127

3.12.2 Identification of Data Needs .................................................................................... 129

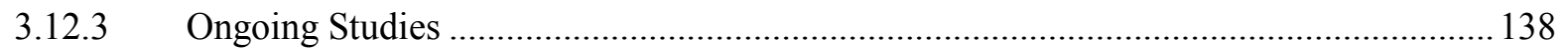




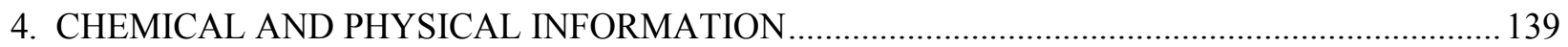

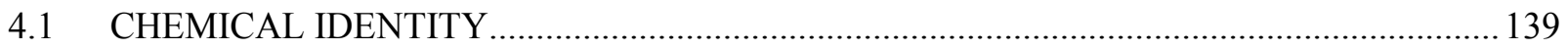

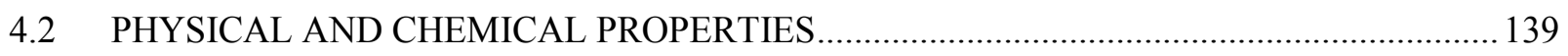

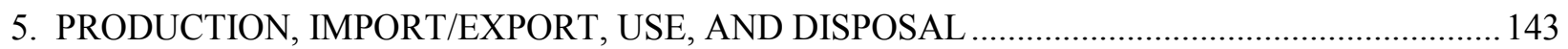

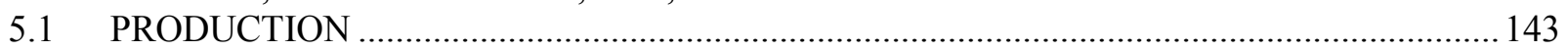

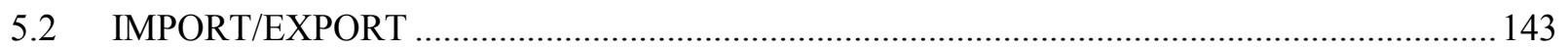

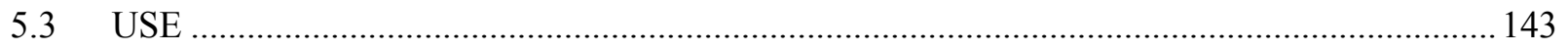

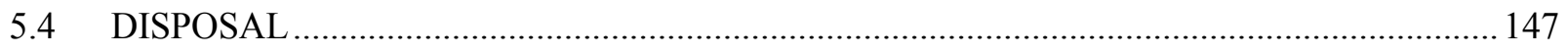

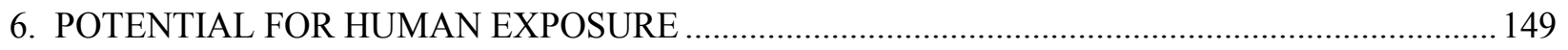

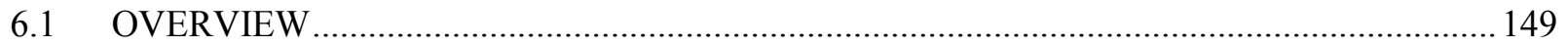

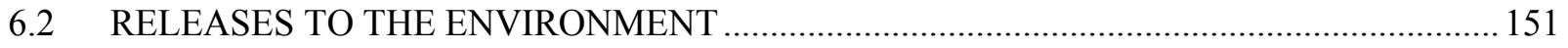

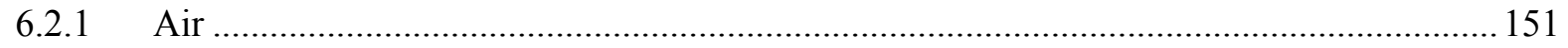

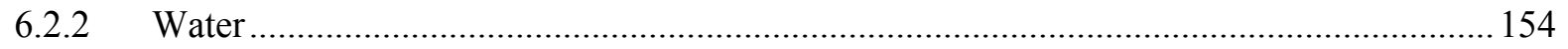

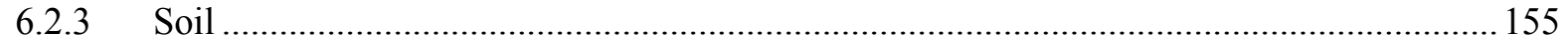

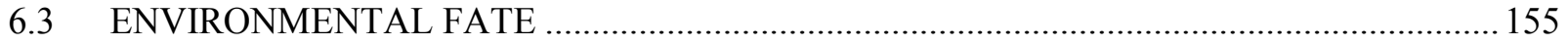

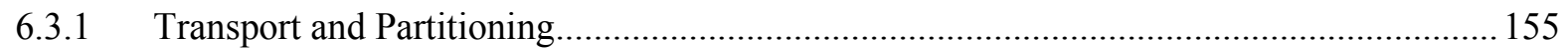

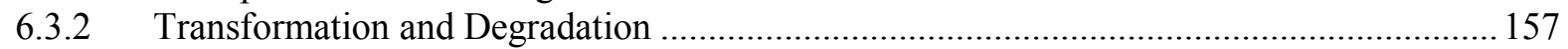

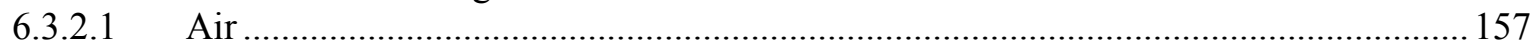

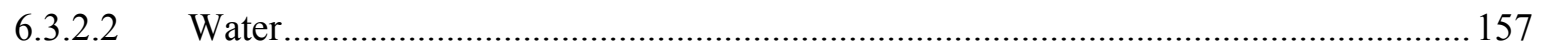

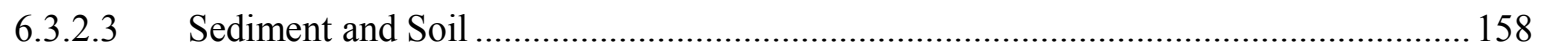

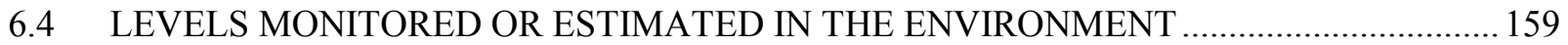

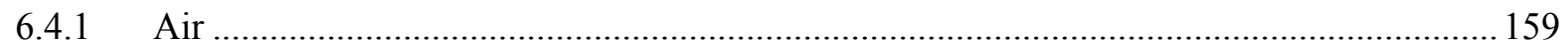

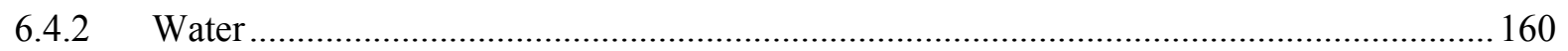

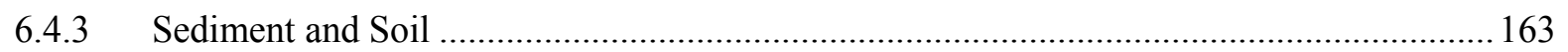

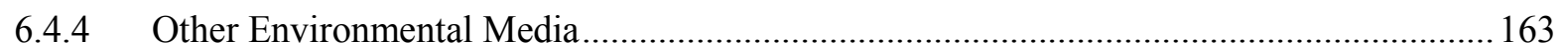

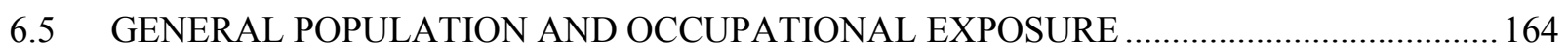

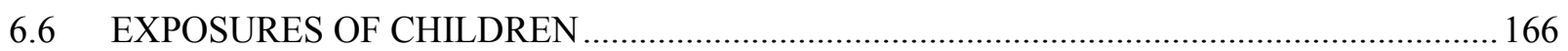

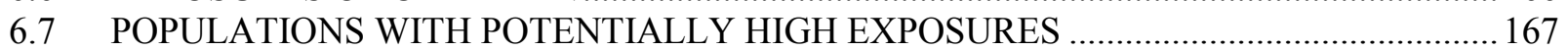

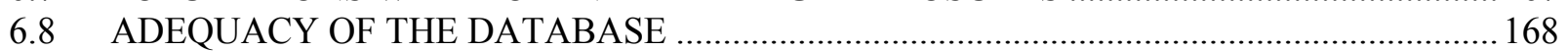

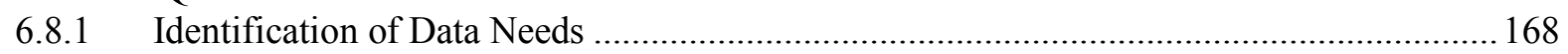

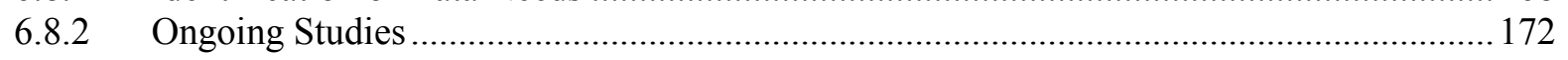

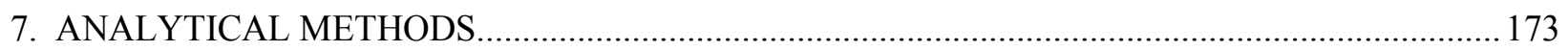

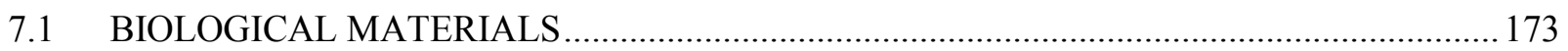

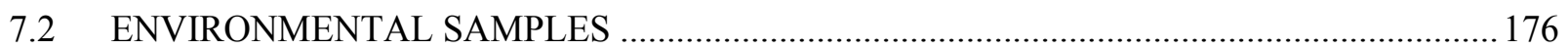

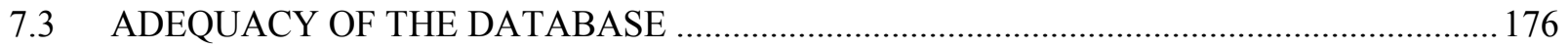

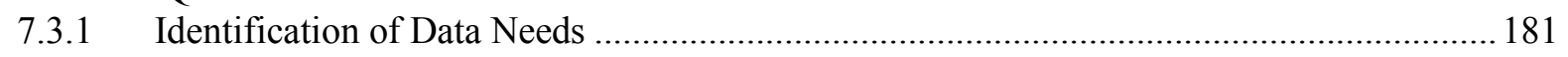

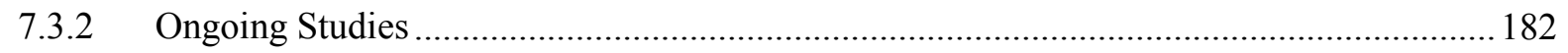

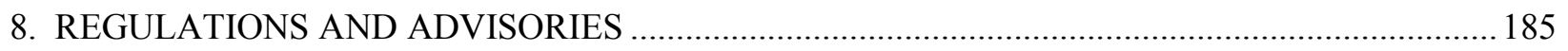

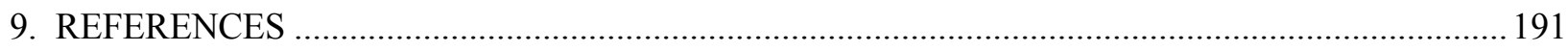

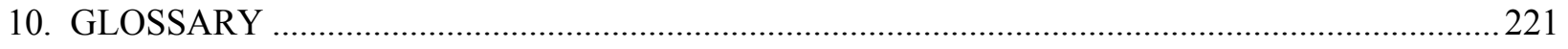




\section{APPENDICES}

A. ATSDR MINIMAL RISK LEVELS AND WORKSHEETS …..................................................... A-1

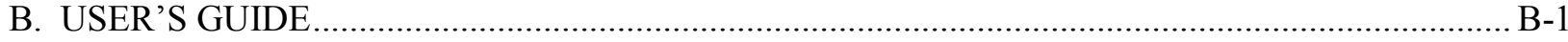

C. ACRONYMS, ABBREVIATIONS, AND SYMBOLS …….........................................................

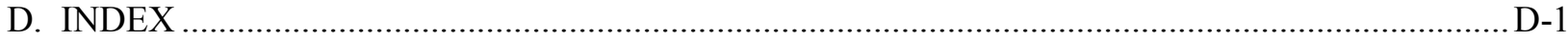




\section{LIST OF FIGURES}

3-1. Levels of Significant Exposure to Phenol - Inhalation ............................................................ 30

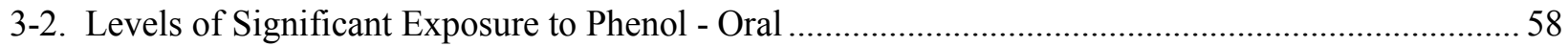

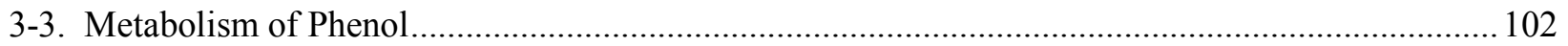

3-4. Conceptual Representation of a Physiologically Based Pharmacokinetic (PBPK) Model for a Hypothetical Chemical Substance.

3-5. Existing Information on Health Effects of Phenol....................................................................... 128

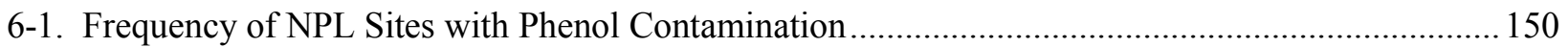


This page is intentionally blank. 


\section{LIST OF TABLES}

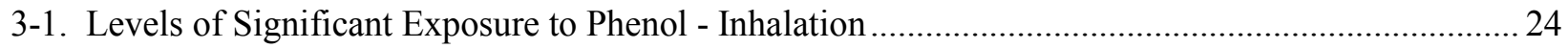

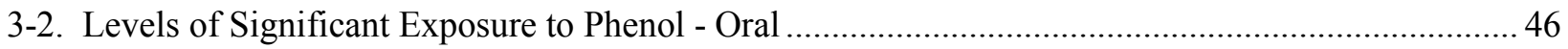

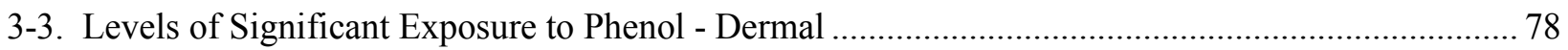

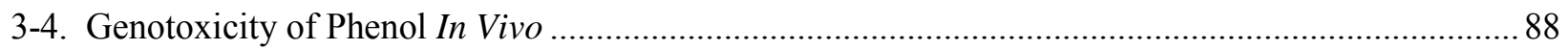

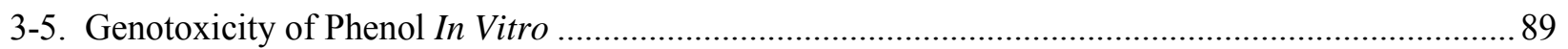

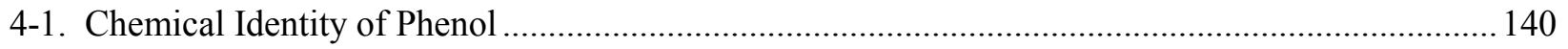

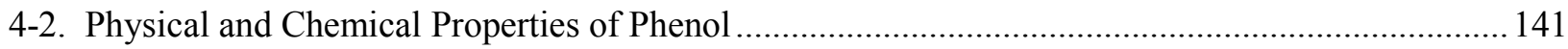

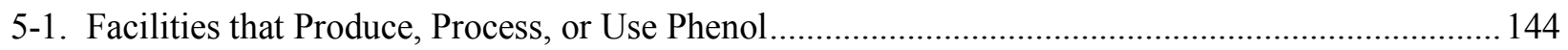

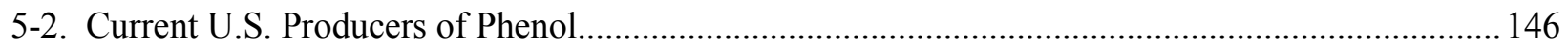

6-1. Releases to the Environment from Facilities that Produce, Process, or Use Phenol ....................... 152

6-2. Estimated Exposure To Phenol At Various Industry Sites ......................................................... 169

7-1. Analytical Methods for Determining Phenol in Biological Samples.......................................... 174

7-2. Analytical Methods for Determining Phenol in Environmental Samples ....................................... 177

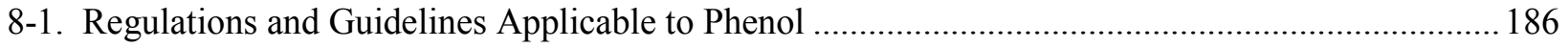


This page is intentionally blank. 


\section{PUBLIC HEALTH STATEMENT}

This public health statement tells you about phenol and the effects of exposure to it.

The Environmental Protection Agency (EPA) identifies the most serious hazardous waste sites in the nation. These sites are then placed on the National Priorities List (NPL) and are targeted for long-term federal clean-up activities. Phenol has been found in at least 595 of the 1,678 current or former NPL sites. Although the total number of NPL sites evaluated for this substance is not known, the possibility exists that the number of sites at which phenol is found may increase in the future as more sites are evaluated. This information is important because these sites may be sources of exposure, and exposure to this substance may be harmful.

When a substance is released either from a large area, such as an industrial plant, or from a container, such as a drum or bottle, it enters the environment. Such a release does not always lead to exposure. You can be exposed to a substance only when you come in contact with it. You may be exposed by breathing, eating, or drinking the substance, or by skin contact.

If you are exposed to phenol, many factors will determine whether you will be harmed. These factors include the dose (how much), the duration (how long), and how you come in contact with it. You must also consider any other chemicals you are exposed to and your age, sex, diet, family traits, lifestyle, and state of health.

\subsection{WHAT IS PHENOL?}

\begin{tabular}{|l|l|}
\hline Description & $\begin{array}{l}\text { Phenol is a colorless-to-white solid when pure. Commercial phenol is a } \\
\text { liquid that evaporates more slowly than water. } \\
\text { Phenol has a distinct odor that is sickeningly sweet and tarry. }\end{array}$ \\
\hline $\begin{array}{l}\text { Uses } \\
\text { - Manufacturing }\end{array}$ & $\begin{array}{l}\text { Phenol is both a manufactured chemical and produced naturally. Large } \\
\text { amounts of phenol are produced in the United States. }\end{array}$ \\
$\begin{array}{l}\text { - Consumer } \\
\text { products }\end{array}$ & $\begin{array}{l}\text { Phenol is used to make plastics. Phenol is also used as a disinfectant in } \\
\text { household cleaning products and in consumer products such as: } \\
\text { - mouthwashes } \\
\text { - gargles } \\
\text { throat sprays }\end{array}$ \\
\hline
\end{tabular}


For more information on the physical and chemical properties of phenol, and its production, disposal and use, see Chapters 4 and 5.

\subsection{WHAT HAPPENS TO PHENOL WHEN IT ENTERS THE ENVIRONMENT?}

\begin{tabular}{|l|l|}
\hline Sources & $\begin{array}{l}\text { Phenol can be found in air and water after release from the manufacture, use, } \\
\text { and disposal of products containing phenol. Phenol in soil is likely to move to } \\
\text { groundwater. }\end{array}$ \\
$\begin{array}{l}\text { Break down } \\
\text { - Air }\end{array}$ & $\begin{array}{l}\text { Phenol is quickly broken down in the air, usually within 1-2 days. } \\
\text { - Water }\end{array}$ \\
$\begin{array}{l}\text { - Soil } \\
\text { Phenol may persist in water for a week or more. }\end{array}$ \\
$\begin{array}{l}\text { Phenol that remains in soil may be broken down by bacteria or other } \\
\text { microorganisms. }\end{array}$
\end{tabular}

For more information on phenol in the environment, see Chapter 6.

\subsection{HOW MIGHT I BE EXPOSED TO PHENOL?}

\begin{tabular}{|l|l|}
\hline Air & $\begin{array}{l}\text { The primary way you can be exposed to phenol is by breathing air } \\
\text { containing it. Releases of phenol into the air occur from: } \\
\text { • industries using or manufacturing phenol } \\
\text { - automobile exhaust } \\
\text { - cigarette smoke, and } \\
\text { - wood burning } \\
\text { Recent data on levels of phenol in air are lacking. }\end{array}$ \\
\hline Water and soil & $\begin{array}{l}\text { Phenol has been detected in surface waters, rainwater, sediments, drinking } \\
\text { water, groundwater, industrial and urban runoff, and at hazardous waste } \\
\text { sites. Phenol in soil is likely to move to groundwater. }\end{array}$ \\
\hline Workplace & $\begin{array}{l}\text { Workers in the following industries may be exposed to phenol: } \\
\text { - petroleum industry } \\
\text { - manufacture of nylon, epoxy resins and polycarbonates, herbicides, wood } \\
\text { preservatives, hydraulic fluids, heavy-duty surfactants, lube-oil additives, } \\
\text { tank linings and coatings, and intermediates for plasticizers and other } \\
\text { specialty chemicals } \\
\text { Exposure occurs through breathing and dermal contact with contaminated } \\
\text { air or by skin contact with products containing phenol. }\end{array}$ \\
\hline
\end{tabular}




\begin{tabular}{|l|l|}
\hline Food & $\begin{array}{l}\text { Low levels of phenol have been found in foods such as smoked summer } \\
\text { sausage, smoked pork belly, mountain cheese, fried bacon, fried chicken, } \\
\text { and black fermented tea. }\end{array}$ \\
\hline $\begin{array}{l}\text { Consumer } \\
\text { products }\end{array}$ & $\begin{array}{l}\text { Dermal contact can occur through the use of general disinfectants and } \\
\text { ointments containing phenol. } \\
\text { Ingestion can occur through the use of products such as throat lozenges or } \\
\text { sore throat sprays that contain phenol. }\end{array}$ \\
\hline
\end{tabular}

For more information on human exposure to phenol, see Chapter 6.

\subsection{HOW CAN PHENOL ENTER AND LEAVE MY BODY?}

\begin{tabular}{|c|c|}
\hline Enter your body & \\
\hline - Inhalation & $\begin{array}{l}\text { When you breathe air containing phenol, most of the phenol will rapidly } \\
\text { enter your body through your lungs. }\end{array}$ \\
\hline - Ingestion & $\begin{array}{l}\text { Phenol in food or water may also rapidly enter your body through the } \\
\text { digestive tract. }\end{array}$ \\
\hline - Dermal contact & $\begin{array}{l}\text { A significant amount may enter through your skin when you come into } \\
\text { contact with phenol vapor, liquid phenol or liquids containing phenol. }\end{array}$ \\
\hline Leave your body & $\begin{array}{l}\text { Once in your body, phenol is transformed into other chemicals called } \\
\text { metabolites. Most of these other chemicals leave your body in the urine } \\
\text { within few days. }\end{array}$ \\
\hline
\end{tabular}

For more information on how phenol enters and leaves the body, see Chapter 3. 


\subsection{HOW CAN PHENOL AFFECT MY HEALTH?}

This section looks at studies concerning potential health effects in animal and human studies.

\begin{tabular}{|c|c|}
\hline $\begin{array}{l}\text { Workers } \\
\text { - Inhalationl } \\
\text { dermal }\end{array}$ & $\begin{array}{l}\text { Long-term exposure to phenol at work has been associated with } \\
\text { cardiovascular disease, but the workers were also exposed to other } \\
\text { chemicals at the same time. }\end{array}$ \\
\hline $\begin{array}{l}\text { General } \\
\text { population } \\
\text { - Oral }\end{array}$ & $\begin{array}{l}\text { Ingestion of liquid products containing concentrated phenol can cause } \\
\text { serious gastrointestinal damage and even death. }\end{array}$ \\
\hline $\begin{array}{l}\text { General } \\
\text { population } \\
\text { - Dermal }\end{array}$ & $\begin{array}{l}\text { Application of concentrated phenol to the skin can cause severe skin } \\
\text { damage. }\end{array}$ \\
\hline $\begin{array}{l}\text { Laboratory } \\
\text { animals } \\
\bullet \text { Inhalation }\end{array}$ & $\begin{array}{l}\text { Short-term exposure to high levels of phenol has caused irritation of the } \\
\text { respiratory tract and muscle twitching in animals. } \\
\text { Longer-term exposure to high levels of phenol caused damaged to the } \\
\text { heart, kidneys, liver, and lungs in animals. }\end{array}$ \\
\hline $\begin{array}{l}\text { Laboratory } \\
\text { animals } \\
\text { - Oral }\end{array}$ & $\begin{array}{l}\text { Drinking water with extremely high concentrations of phenol has caused } \\
\text { muscle tremors, difficulty walking, and death in animals. }\end{array}$ \\
\hline $\begin{array}{l}\text { Laboratory } \\
\text { animals } \\
\text { - Dermal }\end{array}$ & $\begin{array}{l}\text { Short-term application of phenol to the skin has produced blisters and burns } \\
\text { in animals. }\end{array}$ \\
\hline Cancer & $\begin{array}{l}\text { There is no evidence that phenol causes cancer in humans. } \\
\text { The International Agency for Research on Cancer (IARC) and the EPA } \\
\text { determined that phenol is not classifiable as to human carcinogenicity. }\end{array}$ \\
\hline
\end{tabular}

Further information on the health effects of phenol in humans and animals can be found in Chapters 2 and 3. 


\subsection{HOW CAN PHENOL AFFECT CHILDREN?}

This section discusses potential health effects in humans from exposures during the period from conception to maturity at 18 years of age.

\begin{tabular}{|l|l|}
\hline Effects in children & $\begin{array}{l}\text { Vomiting and lethargy were the main symptoms observed in children } \\
\text { following accidental ingestion of a disinfectant containing phenol. We do not } \\
\text { know whether children would be more sensitive than adults to the effects of } \\
\text { phenol. }\end{array}$ \\
\hline Birth defects & $\begin{array}{l}\text { Two studies of women exposed to phenol and other chemicals during } \\
\text { pregnancy did not provide evidence of birth defects. } \\
\text { Some birth defects have been observed in animals born to females exposed } \\
\text { to phenol during pregnancy. This generally occurred at exposure levels that } \\
\text { were also toxic to the mothers. }\end{array}$ \\
\hline Breast milk & There is no information on levels of phenol in human breast milk. \\
\hline
\end{tabular}

\subsection{HOW CAN FAMILIES REDUCE THE RISK OF EXPOSURE TO PHENOL?}

\begin{tabular}{|l|l|}
\hline Tobacco smoke & $\begin{array}{l}\text { Phenol is a component of tobacco smoke. Avoid smoking in enclosed } \\
\text { spaces like inside the home or car in order to limit exposure to children and } \\
\text { other family members. }\end{array}$ \\
\hline $\begin{array}{l}\text { Consumer } \\
\text { products }\end{array}$ & $\begin{array}{l}\text { Household products and over-the-counter medications containing phenol } \\
\text { should be stored out of the reach of young children to prevent accidental } \\
\text { poisonings and skin burns. }\end{array}$ \\
\hline
\end{tabular}




\subsection{IS THERE A MEDICAL TEST TO DETERMINE WHETHER I HAVE BEEN EXPOSED TO PHENOL?}

\begin{tabular}{|l|l|}
\hline $\begin{array}{l}\text { Detecting } \\
\text { exposure }\end{array}$ & $\begin{array}{l}\text { Phenol can be measured in blood and urine. Phenol is a normal constituent } \\
\text { of human urine. }\end{array}$ \\
\hline $\begin{array}{l}\text { Measuring } \\
\text { exposure }\end{array}$ & $\begin{array}{l}\text { A higher-than-normal concentration of phenol in the urine may suggest } \\
\text { recent exposure to phenol or to substances that are converted to phenol in } \\
\text { the body. }\end{array}$ \\
& $\begin{array}{l}\text { The detection of phenol and/or its metabolites in your urine cannot be used } \\
\text { to predict the kind of health effects that might develop from that exposure. }\end{array}$
\end{tabular}

Information about tests for detecting phenol in the body is given in Chapters 3 and 7.

\subsection{WHAT RECOMMENDATIONS HAS THE FEDERAL GOVERNMENT MADE TO PROTECT HUMAN HEALTH?}

The federal government develops regulations and recommendations to protect public health. Regulations can be enforced by law. The EPA, the Occupational Safety and Health Administration (OSHA), and the Food and Drug Administration (FDA) are some federal agencies that develop regulations for toxic substances. Recommendations provide valuable guidelines to protect public health, but cannot be enforced by law. The Agency for Toxic Substances and Disease Registry (ATSDR) and the National Institute for Occupational Safety and Health (NIOSH) are two federal organizations that develop recommendations for toxic substances.

Regulations and recommendations can be expressed as "not-to-exceed" levels. These are levels of a toxic substance in air, water, soil, or food that do not exceed a critical value. This critical value is usually based on levels that affect animals; they are then adjusted to levels that will help protect humans. Sometimes these not-to-exceed levels differ among federal organizations because they used different exposure times (an 8-hour workday or a 24-hour day), different animal studies, or other factors.

Recommendations and regulations are also updated periodically as more information becomes available. For the most current information, check with the federal agency or organization that provides it. 
Some regulations and recommendations for phenol include the following:

\begin{tabular}{|l|l|}
\hline Drinking water & $\begin{array}{l}\text { The EPA has determined that exposure to phenol in drinking water at a } \\
\text { concentration of } 6 \text { milligrams per liter (mg/L) for up to } 10 \text { days is not } \\
\text { expected to cause any adverse effects in a child. }\end{array}$ \\
& $\begin{array}{l}\text { The EPA has determined that lifetime exposure to } 2 \mathrm{mg} / \mathrm{L} \text { phenol in drinking } \\
\text { water is not expected to cause any adverse effects. }\end{array}$ \\
\hline Bottled water & $\begin{array}{l}\text { The FDA has determined that the phenol concentration in bottled drinking } \\
\text { water should not exceed } 0.001 \mathrm{mg} / \mathrm{L} .\end{array}$ \\
\hline Workplace air & $\begin{array}{l}\text { OSHA set a legal limit of } 5 \text { parts per million }(\mathrm{ppm}) \text { phenol in air averaged } \\
\text { over an 8-hour work day. }\end{array}$ \\
\hline
\end{tabular}

For more information on regulations and advisories, see Chapter 8.

\subsection{WHERE CAN I GET MORE INFORMATION?}

If you have any more questions or concerns, please contact your community or state health or environmental quality department, or contact ATSDR at the address and phone number below.

ATSDR can also tell you the location of occupational and environmental health clinics. These clinics specialize in recognizing, evaluating, and treating illnesses that result from exposure to hazardous substances.

Toxicological profiles are also available on-line at www.atsdr.cdc.gov and on CD-ROM. You may request a copy of the ATSDR ToxProfiles ${ }^{\mathrm{TM}} \mathrm{CD}$-ROM by calling the toll-free information and technical assistance number at 1-800-CDCINFO (1-800-232-4636), by e-mail at cdcinfo@cdc.gov, or by writing to:

Agency for Toxic Substances and Disease Registry Division of Toxicology and Environmental Medicine 1600 Clifton Road NE Mailstop F-32

Atlanta, GA 30333

Fax: $\quad 1-770-488-4178$ 
Organizations for-profit may request copies of final Toxicological Profiles from the following:

\author{
National Technical Information Service (NTIS) \\ 5285 Port Royal Road \\ Springfield, VA 22161 \\ Phone: $\quad 1-800-553-6847$ or $1-703-605-6000$ \\ Web site: http://www.ntis.gov/
}




\section{RELEVANCE TO PUBLIC HEALTH}

\subsection{BACKGROUND AND ENVIRONMENTAL EXPOSURES TO PHENOL IN THE UNITED STATES}

Phenol is a naturally occurring and manufactured chemical that is widely distributed in the environment. It is found in various consumer products including throat lozenges, mouthwashes, and antiseptic lotions. The most likely route of exposure to phenol is through dermal contact either in the work environment or at home using ointments and other household products containing phenol.

Phenol is a product of combustion of coal wood and municipal solid waste; therefore, residents near coal and petroleum fueled facilities as well as residents near municipal waste incinerators may have increased exposure to phenol. Phenol is also a product of auto exhaust, and therefore, areas of high traffic likely contain increased levels of phenol. Recent data on concentrations of phenol in air are lacking; it was found at a median concentration of 30 parts per trillion (ppt) in 7 samples from one U.S. urban/suburban site in 1974 and at a median concentration of 5,000 ppt in 83 samples from seven sites between 1974 and 1978. The individual medians of the seven source sites ranged from 520 to 44,000 ppt. Higher phenol concentrations may occur when there is smog or in highly contaminated air.

Phenol has been detected in surface waters, rainwater, sediments, drinking water, groundwater, industrial effluents, urban runoff, and at hazardous waste sites. Levels of up to 1 ppb have been detected in unpolluted groundwater and concentrations ranging from 0.01 to $1 \mathrm{ppb}$ were detected in unpolluted rivers. Phenol has been detected in Lake Huron water at 3-24 ppb and industrial rivers in the United States at 0$5 \mathrm{ppb}$.

Phenol generally does not adhere very strongly to soils and tends to filter rapidly through soil, which may account for the lack of monitoring data, since any phenol released to soils is likely to move to groundwater. In addition, phenol is readily biodegraded under both aerobic and anaerobic conditions, which is expected to attenuate its levels in soil.

Phenol is degraded rapidly in air (half-life of approximately 15 hours), but may persist in water for a somewhat longer period. In soil, phenol will biodegrade rapidly; the half-life in soil is generally $<5$ days.

Although low levels of phenol have been detected in certain foods and tap water, these levels do not constitute major sources of exposure for most people. Phenol has been reported at concentrations of 
7 and $28.6 \mathrm{ppm}$ in smoked summer sausage and smoked pork belly, respectively, and was identified but not quantified in mountain cheese, fried bacon, fried chicken, and black fermented tea.

Since plants can metabolize phenol readily, exposure through eating food derived from plants grown in phenol-containing soil is probably minimal. Due to rapid biodegradation in water and soil, this contamination should be limited. People with contaminated tap water can be exposed from drinking the water or eating foods prepared with it. In addition, inhalation can occur during showering, bathing, and cooking with contaminated water. People can also be exposed to phenol through dermal contact due to bathing or showering with contaminated water.

There are no known unique sources of exposure to children. No reports of phenol in breast milk or baby foods were found. Children are likely to be exposed to phenol through inhalation of contaminated air from wood, coal, and waste incineration as well as from second-hand smoke. Nonsmokers who live with smokers may be exposed to $6-14 \mu \mathrm{g} /$ day of phenol.

\subsection{SUMMARY OF HEALTH EFFECTS}

Information about the health effects of phenol in humans is derived from studies of workers and members of the general population following inhalation, oral, and dermal exposure. These studies indicate that phenol is an irritating and corrosive substance, making the skin and mucosal membranes targets of toxicity, but other effects have also been reported. However, the data for humans exposed to phenol by inhalation or ingestion are inadequate to establish concentration-response relationships, which are needed to identify adverse effects levels. Fatalities due to ingestion or contact with a significant area of the skin have been reported. A minimal lethal oral dose of approximately $70 \mathrm{mg} / \mathrm{kg}$ has been estimated in adults. Other estimates indicate that an oral dose as low as 1,000 $\mathrm{mg}$ could be fatal in humans, but patients occasionally survived doses as high as $65,000 \mathrm{mg}$. Postmortem examination typically showed serious mucosal alterations in the gastrointestinal tract. Other than the skin and mucosal membranes, the liver and cardiovascular system might by considered targets for phenol toxicity. In an epidemiological study of workers from the rubber industry exposed to multiple chemicals (phenol among them), phenol showed the strongest association with mortality due to ischemic heart disease. Electrocardiographic alterations have been reported following acute oral and dermal exposure to phenol, as well as vomiting and lethargy. Studies of populations whose drinking water was contaminated with phenol found increased incidences of nausea and diarrhea, but exposure to chlorophenols may have also occurred. Also, liver effects, as judged by increased serum activities of alanine aminotransferase (ALT) and aspartate amino transferase (AST), 
were reported in a case of prolonged inhalation exposure to phenol and in workers in an oil-refining plant, but exposure to other solvents could not be ruled out in the latter case. An increased incidence of headaches was reported among people who used drinking water contaminated with phenol and probably chlorophenols also. There is no evidence that phenol is a reproductive or developmental toxicant in humans. The Development and Reproductive Toxicant Identification Committee of the California EPA's Office of Environmental Health Hazard Assessment examined the weight of evidence on the reproductive toxicity of phenol and concluded that phenol had not been clearly shown to cause reproductive toxicity.

There is only one modern study of inhalation exposure of animals to phenol. The rest of the inhalation database for phenol is outdated and not useful for risk assessment, although it serves to identify some targets for phenol toxicity. However, no single especially sensitive target emerged from these studies. Short-term (5 minutes) exposure of mice to phenol caused respiratory irritation, as judged by the animals' reflex reduction in respiratory rate; a lowest-observed-effect level (LOEL) was not defined; but the exposure concentration that reduced the respiratory rate by $50 \%$ was $166 \mathrm{ppm}$. In rats exposed nose-only intermittently to concentrations up to $25 \mathrm{ppm}$ for 2 weeks, phenol caused no gross or microscopic alterations in major tissues and organs, including the nasal cavity, but some rats showed an increased incidence in a red nasal discharge possibly due to the irritating properties of phenol. Phenol caused pneumonia, necrosis of the myocardium, centrilobular degeneration, and necrosis of the liver and renal lesions in rabbits and guinea pigs, but not in rats, exposed whole-body intermittently to $26 \mathrm{ppm}$ phenol for intermediate durations. In yet another study in rats, continuous whole-body exposure to $26 \mathrm{ppm}$ phenol for 15 days caused signs of neurological impairment including muscle tremors, twitching, and gait disturbances during the first 3-5 days of exposure. At termination, serum transaminases were elevated suggesting liver damage, but no histological examination was conducted. Neurological effects, including loss of coordination and tremors, were also observed in rats exposed to $234 \mathrm{ppm}$ phenol for 8 hours. In summary, inhaled phenol can affect several organs and tissues and produce neurological effects, but few generalizations can be made from the available studies due to the different exposure protocols used (i.e., nose-only vs. whole-body; intermittent vs. continuous) and incomplete reporting. Toxicokinetics information indicates that phenol is readily absorbed through the skin of humans and animals, so that whole-body exposure may result in considerably more absorbed phenol than in nose-only exposures.

Application of phenol to the skin of animals has caused edema, erythema, necrosis, and death; the cause of death was not provided in the studies available. The effects of phenol on the skin are due to its property to impair the stratum corneum and produce coagulation necrosis by denaturing and precipitating proteins. Lethality is influenced by the surface area exposed as well as the concentration of the applied 
solution. Systemic effects also have been described in animals following dermal exposure to phenol. Rabbits that received a dose of phenol of $24 \mathrm{mg} / \mathrm{cm}^{2} / \mathrm{kg}$ suffered cardiac arrhythmia. Tremors leading to convulsions were reported in rats following application of $107 \mathrm{mg} / \mathrm{kg}$ of phenol to an unspecified surface area.

In contrast to the limited inhalation database, there is an extensive database of oral studies in animals; yet, it is not easy to characterize the toxicity of orally administered phenol. A key factor contributing to the inability to do so is that phenol administered by oral gavage is much more toxic than when it is administered in the drinking water, a phenomenon that is related to the toxicokinetics of phenol. Studies have shown that the toxicity of phenol is correlated with peak blood concentration rather than with total dose, such as the area under the blood concentration curve (AUC). Thus, end points that appear sensitive to phenol administered by oral gavage are not affected by the same total daily dose given via the drinking water.

Results from a 28-day drinking water study in mice provided the lowest effect levels in the oral database for phenol and suggested that hematological, neurochemical, and immunological end points may be particularly sensitive to phenol toxicity. However, since the effects reported in that study occurred at dose levels much lower than in any other study available, these findings should be interpreted with caution until supporting results are available. In one study, phenol induced a significant decrease in red blood cell counts in mice at $\geq 1.8 \mathrm{mg} / \mathrm{kg} / \mathrm{day}$. While this finding could have been due to macrocytosis, the study did not provide enough information to evaluate this possibility. Only two additional studies provided information on hematological parameters after phenol exposure. In pregnant mice, a single gavage dose of $265 \mathrm{mg} / \mathrm{kg}$ of phenol (only dose tested) on gestation day (GD) 13 induced a reduction in the ratio of poly/normochromatic erythrocytes in the bone marrow, whereas phenol administered to rats in doses of up to $320 \mathrm{mg} / \mathrm{kg} /$ day in the drinking water for 10 weeks did not significantly affect a comprehensive number of hematological parameters monitored. Other long-term drinking water studies in rats and mice did not evaluate hematological parameters.

The neurochemical effects caused by phenol in a study also occurred at $\geq 1.8 \mathrm{mg} / \mathrm{kg} /$ day and consisted of alterations in the levels of neurotransmitters in various brain areas. This is difficult to interpret in light of the absence of clinical signs and lack of supporting evidence from other studies. Phenol, however, did induce neurological effects in other studies. Short-term oral gavage administration of doses $\geq 120 \mathrm{mg} / \mathrm{kg} /$ day of phenol caused muscle twitching and tremors in rats and mice, but no effects were observed at $<40 \mathrm{mg} / \mathrm{kg} /$ day. Decreased motor activity was reported in female rats dosed with 
$360 \mathrm{mg} / \mathrm{kg} /$ day of phenol in the drinking water for 13 weeks, but no effects were seen at $107 \mathrm{mg} / \mathrm{kg} / \mathrm{day}$. No neurochemical evaluations were conducted in these studies. Other long-term drinking water studies in rats and mice that used much higher doses of phenol did not observe significant changes in gross or microscopic appearance of the brain of the animals, but no neurological tests or neurochemical evaluations were conducted in these studies.

Phenol caused a significant decrease in antibody response to immunization with sheep red blood cells (SRBC) in mice at $\geq 6.2 \mathrm{mg} / \mathrm{kg} /$ day in a drinking water study. Lymphoproliferative responses to $\mathrm{T}$ and B cell mitogens were also significantly suppressed at $33.6 \mathrm{mg} / \mathrm{kg} /$ day. A study in which the plaqueforming cell assay to SRBC (but not the antibody titer) and lymphoreticular organs of rats exposed to up to $321 \mathrm{mg} / \mathrm{kg} /$ day of phenol in the drinking water for 10 weeks were evaluated found no significant alterations. In another study, a single dose of $224 \mathrm{mg} / \mathrm{kg}$ of phenol administered to rats by oral gavage caused necrosis or atrophy of the spleen or thymus, but no other immunological end point was evaluated. Long-term drinking water studies in rats and mice did not report any significant gross and histological alteration in lymphoreticular organs and tissues at phenol doses $>1,000 \mathrm{mg} / \mathrm{kg} / \mathrm{day}$.

Other effects of phenol observed in oral studies include renal tubular necrosis in rats treated with a single gavage dose of $224 \mathrm{mg} / \mathrm{kg}$ or with $40 \mathrm{mg} / \mathrm{kg} /$ day for 14 days. However, long-term drinking water studies in rats and mice that received much higher doses of phenol do not suggest that the kidney is a particularly sensitive target for phenol. Phenol also induced decreases in body weight in rats and mice in 13-week and 2-year drinking water studies that were associated with significant reductions in water consumption due probably to poor palability. Phenol reduced body weight gain in pregnant mice treated by oral gavage with $280 \mathrm{mg} / \mathrm{kg} / \mathrm{day}$, a dose level that also caused frank neurotoxicity. Doses of $120 \mathrm{mg} / \mathrm{kg} / \mathrm{day}$ of phenol administered to pregnant rats during GDs 6-15 using a divided dosing protocol to minimize the adverse effects of a bolus dose caused a significant reduction in weight gain in the dams; the no-observedadverse-effect level (NOAEL) was $60 \mathrm{mg} / \mathrm{kg} /$ day. The latter findings suggest that weight gain during pregnancy is a sensitive end point for phenol toxicity and the dose of $120 \mathrm{mg} / \mathrm{kg} /$ day was the lowest LOAEL in acute-duration oral studies in which no overt signs of toxicity (other than reduced maternal weight gain) were observed following administration of phenol.

Phenol has induced developmental effects in rodents, but, with one exception, it appears that this occurs at dose levels that also affect the mothers. In one study, doses of $120 \mathrm{mg} / \mathrm{kg} / \mathrm{day}$ of phenol on GDs 6-15 produced a $7 \%$ decrease in average fetal body weight in the absence of maternal effects. In the study that used the divided dosing protocol mentioned above, there were no developmental effects at 
$120 \mathrm{mg} / \mathrm{kg} /$ day, but decreased ossification sites were seen at the highest dose level, $360 \mathrm{mg} / \mathrm{kg} / \mathrm{day}$. In a two-generation reproductive study in which the parental generation received doses of up to 301$321 \mathrm{mg} / \mathrm{kg} /$ day of phenol via the drinking water, decreased pup weight and percent live pups on postnatal day 4 was reported at a dose level that also significantly decreased maternal water consumption, including during gestation and lactation. In pregnant mice, doses of $280 \mathrm{mg} / \mathrm{kg} /$ day on GDs 6-15 produced a significant decrease in fetal weight and also caused tremors and ataxia in the dams.

A study of phenol-exposed wood industry workers reported a small, nonsignificant excess of respiratory cancers and a study of phenol production workers reported a small, non-significant excess of Hodgkin's disease and of lung, esophageal, rectal, and kidney cancers. However, the interpretation of these findings is complicated due to lack of dose-response and potential for confounding. Phenol has been tested for carcinogenicity in long-term drinking water bioassays in rats and mice. Statistically significant increased incidences of pheochromocytomas of the adrenal gland and leukemia or lymphomas were observed in male rats exposed to the low dose of phenol, but not to the high dose of phenol. No significant effects were seen in female rats or in mice. Phenol has consistently been found to be a promoter in initiationpromotion studies in mouse skin. Based on inadequate evidence in humans and in animals, EPA assigned phenol to Group D, not classifiable as to human carcinogenicity. Under updated guidelines, the data regarding carcinogenicity of phenol are: "inadequate for an assessment of human carcinogenic potential."

\subsection{MINIMAL RISK LEVELS (MRLS)}

Estimates of exposure levels posing minimal risk to humans (MRLs) have been made for phenol. An MRL is defined as an estimate of daily human exposure to a substance that is likely to be without an appreciable risk of adverse effects (noncarcinogenic) over a specified duration of exposure. MRLs are derived when reliable and sufficient data exist to identify the target organ(s) of effect or the most sensitive health effect(s) for a specific duration within a given route of exposure. MRLs are based on noncancerous health effects only and do not consider carcinogenic effects. MRLs can be derived for acute, intermediate, and chronic duration exposures for inhalation and oral routes. Appropriate methodology does not exist to develop MRLs for dermal exposure.

Although methods have been established to derive these levels (Barnes and Dourson 1988; EPA 1990), uncertainties are associated with these techniques. Furthermore, ATSDR acknowledges additional uncertainties inherent in the application of the procedures to derive less than lifetime MRLs. As an example, acute inhalation MRLs may not be protective for health effects that are delayed in development 
or are acquired following repeated acute insults, such as hypersensitivity reactions, asthma, or chronic bronchitis. As these kinds of health effects data become available and methods to assess levels of significant human exposure improve, these MRLs will be revised.

\section{Inhalation MRLs}

An acute-duration inhalation MRL was not derived for phenol due to inadequacies of the limited database available. The database includes a few animal studies of limited scope (Aranyi et al. 1986; De Ceaurriz et al. 1981; Flickinger 1976) and a well-conducted study that used modern methodology to evaluate a number of relevant end points (Hoffman et al. 2001). No relevant human studies were located. In the animal studies, a target for phenol toxicity was not clearly defined; however, for an irritant substance such as phenol, it is reasonable to assume that portals of entry, such as the respiratory tract, could be potential targets. Of the studies mentioned above, only Hoffman et al. (2001) conducted a careful evaluation of the respiratory tract. Hoffman et al. (2001) exposed rats to various exposure levels for 2 weeks and evaluated a number of end points including histopathology, hematology, and clinical chemistry and reported no adverse effects. De Ceaurriz et al. (1981) exposed mice to various concentrations of phenol in air for 5 minutes and determined an $\mathrm{RD}_{50}$ (concentration that reduced the respiratory rate by $50 \%$, a protective reflex response in rodents) of $166 \mathrm{ppm}$. Aranyi et al. (1986) also exposed mice to $5 \mathrm{ppm}$ phenol 3 hours/day for 5 days and reported no significant changes in susceptibility to airborne bacterial agents relative to mice exposed to filtered air. Flickinger (1976) observed loss of coordination and tremors in rats exposed to $234 \mathrm{ppm}$ phenol for 8 hours; a 1-hour exposure was without effect. No other exposure concentration was tested and no control group was used. Fourteen days later, the rats were sacrificed and subjected to gross necropsy. Flickinger (1976) indicated that no gross lesions were observed, but the scope of the examination was not specified. Of all the studies available, the one conducted by Hoffman et al. (2001) is the most complete, better-reported, and used modern methodology, but, as indicated above, because no adverse effects were reported, it is not a suitable basis for an MRL.

An intermediate-duration inhalation MRL was not derived for phenol due to lack of adequate data. No relevant human data were located and the available animal studies had numerous limitations including poor control of exposure levels, unclear scope of the evaluations, and limited reporting. The intermediate-duration database consists of only three studies (Dalin and Kristoffersson 1974; Deichmann et al. 1944; U.S. Air Force 1961). Dalin and Kristoffersson (1974) exposed a small number of rats to 0 or 26 ppm phenol continuously for 15 days and reported mild motor disorders (impaired balance, abnormal gait, muscle twitching) during the first few days of exposure. At termination, the activities of serum 
transaminases were significantly increased indicating liver damage, but no histopathology examination was conducted. Because the exposure chamber was not of modern design, there is some uncertainty as to the actual exposure levels. Deichmann et al. (1944) exposed guinea pigs, rats, and rabbits intermittently for 6-12 weeks to a concentration of phenol in air that apparently could not be controlled with any precision, but could have ranged from 26 to $52 \mathrm{ppm}$. No controls were used and no actual data were presented; the paper contains only a narrative of the results. Exposure to phenol caused serious histological alterations in the lungs, heart, liver, and kidneys in rabbits and guinea pigs, but no significant changes were reported in rats. U.S. Air Force (1961) exposed monkeys, rats, and mice continuously to 0 or 5 ppm phenol for 90 days. No information was provided regarding the frequency of monitoring the test atmosphere, but the concentration of phenol was reported to remain in the range of 4.5-5.5 ppm after the first few days of the experiment. Although the report indicates that there were no significant histological alterations in organs and tissues, incomplete reporting of the results suggests that there may have been some lung, liver, and kidney pathology. In addition, no data were presented to support the assertion that there were no effects on hematology (three species), blood chemistry (monkeys only), urinalysis (three species), and kidney function tests (monkeys and rats).

A chronic-duration inhalation MRL for phenol was not derived due to lack of data for this duration. Occupational studies in humans are limited by lack of exposure data and simultaneous exposure to multiple chemicals. No chronic inhalation study in animals was located.

\section{Oral MRLs}

No reliable human data were located for derivation of oral MRLs for phenol. As mentioned in Section 2.2, effects of phenol administered to animals by oral gavage are different than those observed in drinking water studies. Administration of phenol by oral gavage, as was done in almost all acute-duration oral studies, results in adverse effect levels that are much lower than those identified in drinking water studies. For example, tremors were reported in rats administered a single gavage dose of $120 \mathrm{mg} / \mathrm{kg}$ (Berman et al. 1995) and in pregnant mice administered $140 \mathrm{mg} / \mathrm{kg} /$ day during gestation (NTP 1983b), but no adverse neurological signs were reported in rats administered $360 \mathrm{mg} / \mathrm{kg} /$ day in the drinking water for 13 weeks (Beyrouty 1998) or in rats or mice administered phenol in the drinking water in doses exceeding $700 \mathrm{mg} / \mathrm{kg} /$ day for up to 103 weeks (NCI 1980). This differential toxicity is related to the toxicokinetics of phenol. A study by Hiser et al. (1994) showed that the toxicity of phenol is correlated with peak blood concentration rather than with total dose, such as the AUC. Hiser et al. (1994) observed that rats given phenol by oral gavage developed a cluster of behaviors that the investigators termed 
"phenol twitching behavior" consisting of tremors, sudden jerks, hyper-reactivity to stimulus, and excessive blinking, none of which occurred in groups dosed via the drinking water. Hiser et al. (1994) also noticed that the twitching behavior developed almost immediately after gavage dosing, a time that also coincided with peak blood levels of phenol, and disappeared by 37 minutes after dosing. Also, for a given daily dose, peak levels of phenol in blood were much higher following gavage dosing than following continuous administration in the drinking water. Additional information that supports the idea of toxicity being associated with peak blood levels of phenol was provided by experiments done by NTP (1983a). These investigators treated pregnant rats with phenol by gavage in different volumes during GDs 6-15. In a group dosed with $125 \mathrm{mg} / \mathrm{kg} /$ day in a volume of $1 \mathrm{~mL} / \mathrm{kg}, 7 \mathrm{of} 10$ rats died. Deaths were preceded by dose-related signs of toxicity, including tremors, convulsions, and respiratory distress, and necropsy revealed mottled liver and congested lungs. However, in a group treated with $160 \mathrm{mg} / \mathrm{kg} / \mathrm{day}$ in a volume of $5 \mathrm{~mL} / \mathrm{kg}$ only one of six rats died, as a larger dosing volume would be expected to decrease the absorption rate. Based on the information discussed in this paragraph, only drinking water studies and divided dose gavage studies were considered appropriate for MRL derivation.

- An MRL of $1 \mathrm{mg} / \mathrm{kg} /$ day has been derived for acute-duration oral exposure (1-14 days) to phenol.

All of the acute-duration oral studies available administered phenol to the animals by gavage. As indicated in the preceding paragraph, dosing volume in oral gavage studies is important in the manifestation of phenol toxicity. Acute-duration studies that used a relatively low dosing volume of $1 \mathrm{~mL} / \mathrm{kg}$ are those by Berman et al. (1995), Moser et al. (1995), and Narotsky and Kavlock (1995). These studies were not considered for MRL derivation even though they identified adverse effects at dose levels lower than studies that used divided gavage dosing or drinking water studies. The remaining database is essentially limited to two developmental studies, which were considered for MRL derivation. In one of these studies, rats were gavaged with phenol in doses of up to $120 \mathrm{mg} / \mathrm{kg} /$ day in a dosing volume of $5 \mathrm{~mL} / \mathrm{kg}$ during GDs 6-15 (NTP 1983a). There was no maternal toxicity, but mean fetal body weight at this dose level was approximately 7\% lower than controls. However, since historical control data showed that the concurrent control fetal weight for the CD rat was much higher (22\%) than the historical control weight and a larger litter size in the high-dose group may have contributed to the smaller fetal weight in the high-dose group, the dose of $120 \mathrm{mg} / \mathrm{kg} /$ day can be considered an equivocal LOAEL for developmental effects; the NOAEL was $60 \mathrm{mg} / \mathrm{kg} / \mathrm{day}$. In the other developmental study, which used a divided dosing protocol and a dosing volume of $10 \mathrm{~mL} / \mathrm{kg}$, there was a dose-related decrease in maternal body weight gain during treatment days and beyond, which achieved statistical significance at $120 \mathrm{mg} / \mathrm{kg} /$ day (York 1997). Maternal body weight was also reduced, but differences with control 
achieved statistical significance only at $360 \mathrm{mg} / \mathrm{kg} / \mathrm{day}$ (see details below). No fetal toxicity was seen at $120 \mathrm{mg} / \mathrm{kg} /$ day. The decrease in maternal body weight gain during gestation was the most sensitive end point and the dose level of $120 \mathrm{mg} / \mathrm{kg} /$ day is considered a LOAEL; the NOAEL is $60 \mathrm{mg} / \mathrm{kg} / \mathrm{day}$. Since the York (1997) study identified the most sensitive end point and utilized a dosing protocol that resembles more closely a potential environmental exposure scenario to phenol, it was selected as the principal study for the derivation of an acute-duration oral MRL for phenol.

In the York (1997) study, groups of pregnant Sprague-Dawley rats (25/dose group) were dosed 3 times daily with $0,20,40$, or $120 \mathrm{mg}$ phenol $/ \mathrm{kg}$ in water (total daily doses of $0,60,120$, or $360 \mathrm{mg} / \mathrm{kg}$ ) by gavage on GDs 6-15; the dosing volume was $10 \mathrm{~mL} / \mathrm{kg}$. Maternal end points evaluated included clinical signs, body weight, and food consumption. Dams were also observed for abortions and premature deliveries. Dams were sacrificed on GD 20 and a gross necropsy was conducted. The uterus was examined for pregnancy, number and distribution of implantations, live and dead fetuses, and early and late resorptions. Fetuses were weighed and examined for sex and gross external alterations. Half of the fetuses were examined for soft tissue alterations and the remaining fetuses were examined for skeletal alterations. One dam in the $360 \mathrm{mg} / \mathrm{kg} /$ day group died on GD 11 and the death was attributed to phenol treatment. Clinical signs considered treatment-related included excess salivation and tachypnea in rats exposed to $360 \mathrm{mg} / \mathrm{kg} /$ day. Gross necropsy of the dams did not reveal any treatment-related alterations. In the $120 \mathrm{mg} / \mathrm{kg}$ /day group, maternal body weight gain was significantly reduced for GDs 6-16 (11\%) and for GDs 12-16 (19\%), whereas in the $360 \mathrm{mg} / \mathrm{kg} /$ day group, body weight gain was reduced 38\% for GDs 6-16. Maternal final body weight in the $360 \mathrm{mg} / \mathrm{kg} /$ day group was reduced, but $<10 \%$ relative to controls. Food consumption was reduced in the $360 \mathrm{mg} / \mathrm{kg} /$ day group by $16 \%$ for GDs $6-20$ and by $15 \%$ for GDs $0-20$; in the $120 \mathrm{mg} / \mathrm{kg}$ /day group, food consumption for GDs 6-16 was reduced $11 \%$. Fetal body weight at the $360 \mathrm{mg} / \mathrm{kg} /$ day level was reduced 5-7\% relative to controls. There was a significant decrease in ossification sites on the hindlimb metatarsals in the $360 \mathrm{mg} / \mathrm{kg} /$ day group, which was considered of minimal biological significance. At the 120 and $360 \mathrm{mg} / \mathrm{kg} / \mathrm{day}$ dose levels, there were increases in litters with fetuses with "any alteration" and with "any variation", but neither reached statistical significance and there were no clear dose-response relationships. There were no significant effects on corpora lutea, implantations, litter sizes, live fetuses, early and late resorptions, or percent resorbed conceptuses. Based on decreased fetal body weight and delayed ossification, the dose of $360 \mathrm{mg} / \mathrm{kg} /$ day is a LOAEL for developmental effects; the NOAEL is $120 \mathrm{mg} / \mathrm{kg} / \mathrm{day}$. Based on decreased weight gain during gestation, the dose of $120 \mathrm{mg} / \mathrm{kg} /$ day is a LOAEL for decreased maternal body weight gain; the NOAEL is $60 \mathrm{mg} / \mathrm{kg} / \mathrm{day}$. 
Data from York (1997) were analyzed using the BMD approach for MRL derivation. BMD models in the EPA Benchmark Dose Software (BMDS version 2.0) (linear, polynomial, power, and Hill models) were fit to the maternal body weight gain data to determine potential points of departure for the MRL (details of the modeling are presented in Appendix A). The linear model with homogeneous variance (which was identical to the power model), was selected because it was the simplest model and provided the best fit. In the absence of a clear criteria as to what level of change in weight gain during pregnancy should be considered adverse, the BMR was defined as a change in mean body weight gain equal to one standard deviation from the control mean (EPA 2000c). The corresponding BMD was $152 \mathrm{mg} / \mathrm{kg} / \mathrm{day}$; the corresponding benchmark dose limit (BMDL) was $125 \mathrm{mg} / \mathrm{kg} /$ day. Applying an uncertainty factor of 100 (10 for animal to human extrapolation and 10 for human variability) to the BMDL results in an acuteduration oral MRL of $1 \mathrm{mg} / \mathrm{kg} /$ day.

An intermediate-duration oral MRL for phenol was not derived. Several studies are available that provide information on the effects of phenol following intermediate-duration exposure and all of them used drinking water to administer the test material. With the exception of one study (Hsieh et al. 1992, see below), doses tested in intermediate-duration oral studies were higher than doses tested in acute-duration oral studies. A 13-week drinking water study in rats and mice evaluated clinical signs and gross and microscopic appearance of a number of organs and tissues and found little evidence of toxicity (NCI 1980). Reduction in body weight gain was observed in both rats and mice at the highest dose levels tested $(1,556 \mathrm{mg} / \mathrm{kg} /$ day in rats, $2,468 \mathrm{mg} / \mathrm{kg} /$ day in mice $)$, which was most likely due to significant decreases in water consumption. Also available is a two-generation reproduction study that found no evidence of reproductive effects in male and female rats (301 and $321 \mathrm{mg} / \mathrm{kg} /$ day, respectively), but reported decreased pup weight and reduced viability at 301/321 mg/kg/day (Ryan et al. 2001). Significantly reduced water consumption was also reported in the Ryan et al. (2001) study, particularly in the $301 / 321 \mathrm{mg} / \mathrm{kg} /$ day males and females. A specialized 13-week neurotoxicity study in rats reported decreased motor activity in females dosed with $360 \mathrm{mg} / \mathrm{kg} /$ day, but not with $107 \mathrm{mg} / \mathrm{kg} /$ day (Beyrouty 1998). However, the most significant findings among the intermediate-duration database were reported in a 28-day study in mice (Hsieh et al. 1992). These investigators found hematological and neurochemical effects in mice at $1.8 \mathrm{mg} / \mathrm{kg} /$ day and immunological effects at $\geq 6.2 \mathrm{mg} / \mathrm{kg} /$ day. Hsieh et al. (1992) dosed CD-1 mice (five per dose group) with phenol in the drinking water for 28 days. At termination, there was a dose-related decrease in red cell counts, statistically significant at all dose levels. The hematocrit was decreased only at the highest dose level. In the absence of a change in hematocrit, the decrease in red blood cells may have been due to macrocytosis, but the study did not provide sufficient information to evaluate this possibility. Ryan et al. (2001), in a two-generation study, conducted a comprehensive 
evaluation of hematological parameters in rats exposed to up to $321 \mathrm{mg} / \mathrm{kg} /$ day for 10 weeks and found no significant alterations. Similarly, in the inhalation experiments of U.S. Air Force (1961) in mice exposed continuously for 90 days, no hematological alterations were observed. Hsieh et al. (1992) also reported significant dose-related alterations in various neurotransmitters in the brain (i.e., dopamine, norepinephrine). In the absence of clinical effects, this is difficult to interpret. Beyrouty (1998) exposed rats to doses of $308 \mathrm{mg} / \mathrm{kg} /$ day of phenol for 13 weeks and found no significant neurological alterations, although neurochemical evaluations were not conducted. Hsieh et al. (1992) also found a significant decrease in antibody response to immunization with SRBCs at $\geq 6.2 \mathrm{mg} / \mathrm{kg} / \mathrm{day}$, detected by two different assays (plaque-forming cell assay and antibody titer). At the highest dose level tested, $33.6 \mathrm{mg} / \mathrm{kg} / \mathrm{day}$, lymphoproliferative responses to $\mathrm{T}$ and $\mathrm{B}$ cell mitogens were also significantly suppressed. Ryan et al. (2001) also conducted the plaque-forming cell assay to SRBC (but not the antibody titer) and evaluated lymphoreticular organs of rats in the two-generation study and found no significant alterations, although it is not uncommon to find differences in immune responses between rats and mice. The Hsieh et al. (1992) study was not used for derivation of an intermediate-duration oral MRL largely due to the unconfirmed nature of findings observed at relatively very low doses and because only five mice comprised each dose group. Hsieh's findings need to be replicated before the data can be used for risk assessment.

A chronic-duration oral MRL for phenol was not derived. The only chronic-duration animal studies are the NCI (1980) 103-week studies in rats and mice. NCI (1980) evaluated clinical signs, organ weights, and gross and microscopic appearance of organs and tissues. The lowest doses tested were $322 \mathrm{mg} / \mathrm{kg} /$ day in rats and $590 \mathrm{mg} / \mathrm{kg} /$ day in mice. Under the conditions of the study, phenol showed essentially no systemic toxicity, but neither hematology nor clinical chemistry tests were conducted. The only reported effect was a significant decrease in body weight in male $(\geq 322 \mathrm{mg} / \mathrm{kg} /$ day $)$ and female $(\geq 721 \mathrm{mg} / \mathrm{kg} /$ day) rats associated with significant decreases in water intake; food consumption was comparable among all groups. It would not be appropriate to use the LOAEL of $322 \mathrm{mg} / \mathrm{kg} / \mathrm{day}$ as the basis for an MRL since the effect (reduced final body weight) was likely due to decreased water intake. An additional reason for not deriving a chronic-duration oral MRL for phenol is the intermediate data from Hsieh et al. (1992) suggesting that immunosuppression may be the most sensitive effect, which leaves open the possibility that it could do the same in longer-term studies. Tests of immunocompetence were not conducted in the standard 2-year bioassays available. 


\section{HEALTH EFFECTS}

\subsection{INTRODUCTION}

The primary purpose of this chapter is to provide public health officials, physicians, toxicologists, and other interested individuals and groups with an overall perspective on the toxicology of phenol. It contains descriptions and evaluations of toxicological studies and epidemiological investigations and provides conclusions, where possible, on the relevance of toxicity and toxicokinetic data to public health.

It should be noted that phenol is the simplest form, or parent compound, of the class of chemicals commonly referred to as phenols or phenolics, many of which are natural substances widely distributed throughout the environment. There is some confusion in the literature as to the use of the term 'phenol'; in some cases, it has been used to refer to a particular phenolic compound that is more highly substituted than the parent compound (Doan et al. 1979), whereas in other cases, it has been used to refer to the class of phenolic compounds (Beveridge 1997). This chapter, however, addresses only those health effects that can be directly attributable to the parent compound, monohydroxybenzene, or phenol. As Deichmann and Keplinger (1981) note: "It cannot be overemphasized that the structure-activity relationships of phenol and phenol derivatives vary widely, and that to accept the properties of individual phenolic compounds as being those of phenol is a misconception and leads to error and confusion."

A glossary and list of acronyms, abbreviations, and symbols can be found in Appendix C at the end of this profile.

\subsection{DISCUSSION OF HEALTH EFFECTS BY ROUTE OF EXPOSURE}

To help public health professionals and others address the needs of persons living or working near hazardous waste sites, the information in this section is organized first by route of exposure (inhalation, oral, and dermal) and then by health effect (death, systemic, immunological, neurological, reproductive, developmental, genotoxic, and carcinogenic effects). These data are discussed in terms of three exposure periods: acute (14 days or less), intermediate (15-364 days), and chronic (365 days or more).

Levels of significant exposure for each route and duration are presented in tables and illustrated in figures. The points in the figures showing no-observed-adverse-effect levels (NOAELs) or lowestobserved-adverse-effect levels (LOAELs) reflect the actual doses (levels of exposure) used in the studies. LOAELs have been classified into "less serious" or "serious" effects. "Serious" effects are those that 
evoke failure in a biological system and can lead to morbidity or mortality (e.g., acute respiratory distress or death). "Less serious" effects are those that are not expected to cause significant dysfunction or death, or those whose significance to the organism is not entirely clear. ATSDR acknowledges that a considerable amount of judgment may be required in establishing whether an end point should be classified as a NOAEL, "less serious" LOAEL, or "serious" LOAEL, and that in some cases, there will be insufficient data to decide whether the effect is indicative of significant dysfunction. However, the Agency has established guidelines and policies that are used to classify these end points. ATSDR believes that there is sufficient merit in this approach to warrant an attempt at distinguishing between "less serious" and "serious" effects. The distinction between "less serious" effects and "serious" effects is considered to be important because it helps the users of the profiles to identify levels of exposure at which major health effects start to appear. LOAELs or NOAELs should also help in determining whether or not the effects vary with dose and/or duration, and place into perspective the possible significance of these effects to human health.

The significance of the exposure levels shown in the Levels of Significant Exposure (LSE) tables and figures may differ depending on the user's perspective. Public health officials and others concerned with appropriate actions to take at hazardous waste sites may want information on levels of exposure associated with more subtle effects in humans or animals (LOAELs) or exposure levels below which no adverse effects (NOAELs) have been observed. Estimates of levels posing minimal risk to humans (Minimal Risk Levels or MRLs) may be of interest to health professionals and citizens alike.

A User's Guide has been provided at the end of this profile (see Appendix B). This guide should aid in the interpretation of the tables and figures for Levels of Significant Exposure and the MRLs.

\subsubsection{Inhalation Exposure}

\subsubsection{Death}

A cohort mortality study of workers in five formaldehyde-resin manufacturing facilities was conducted to evaluate whether excess mortality could be attributed to occupational exposure to phenol (Dosemeci et al. 1991). Workers (exposed and non-exposed) had a mortality rate, from all causes, similar to that of the general U.S. population. Compared to either the general population or unexposed workers, exposed workers had small statistically non-significant excesses in mortality due to Hodgkin's disease (standardized mortality ratio [SMR], 1.7; 95\% confidence interval [CI], 0.8-3.1) and esophageal (SMR, 1.6; 95\% CI, 0.9-2.6), renal (SMR, 1.3; 95\% CI, 0.7-2.1), and rectal (SMR, 1.4; 95\% CI, 0.8-2.2) 
cancers. They also had small reductions in mortality due to: cancer of the stomach, testes, pancreas, buccal cavity/pharynx and brain; lymphosarcoma; liver cirrhosis; emphysema; diseases of the cardiovascular, circulatory, and digestive systems; motor vehicle accidents; and all accidents. The ambiguity of these data, as well as the fact that dose-related trends occurred only for those diseases showing reductions in mortality, makes it difficult to assess the impact on mortality of long-term occupational exposure to phenol.

Deichmann et al. (1944) exposed guinea pigs, rabbits, and rats to phenol vapor at levels ranging from 26 to 52 ppm for 28-88 days. After 28 days of exposure, 5 of 12 guinea pigs died, but no deaths occurred in rabbits or rats. Since only a range was given for the exposure level, the exact level of phenol in air that resulted in death of guinea pigs was not established and may be as low as $26 \mathrm{ppm}$ or as high as $52 \mathrm{ppm}$. Interpretation of this study is further complicated by an apparent lack of controls. However, since the effects observed in guinea pigs and rabbits (described in subsequent sections in Chapter 3) were so severe, it is difficult to ascribe the mortality to any source other than the phenol exposure. The lower limit of the exposure range, 26 ppm, is recorded as a serious LOAEL in Table 3-1 and plotted in Figure 3-1. No deaths were reported in Rhesus monkeys, rats, or mice exposed to $5 \mathrm{ppm}$ phenol continuously for 90 days (U.S. Air Force 1961).

\subsubsection{Systemic Effects}

The highest NOAEL values and all LOAEL values from each reliable study for systemic effects in each species and duration category are recorded in Table 3-1 and plotted in Figure 3-1.

Respiratory Effects. Slight increases in mortality associated with respiratory cancers were seen in two epidemiological studies of workers exposed to phenol (Dosemeci et al. 1991; Kauppinen et al. 1986). However, after adjusting for smoking-related behavior, these increases became nonsignificant in the Kauppinen et al. (1986) study, and neither study showed a dose-related trend; thus, the relevance of these findings to respiratory disease per se is somewhat uncertain. Indeed in the latter study, there were slight, yet nonsignificant reductions in mortality associated with emphysema among exposed workers, leading the investigators to suggest that exposure to phenol could have a protective effect for diseases involving free radical damage.

A case-control study of office workers was conducted by Baj et al. (1994) to evaluate the risks of chronic exposures to "inhaled formaldehyde, phenol, and isomers of organic chlorohydrocarbons from Ksylamit ${ }^{\mathrm{TM}}$..." which is a widely used liquid wood preservative. It should be noted that in the report, 
Table 3-1 Levels of Significant Exposure to Phenol - Inhalation

\begin{tabular}{|c|c|c|c|c|}
\hline $\begin{array}{l}\text { Key to } \\
\text { Figure }\end{array}$ & $\begin{array}{l}\text { Species } \\
\text { (Strain) }\end{array}$ & $\begin{array}{c}\text { Exposurel } \\
\text { Duration/ } \\
\text { Frequency } \\
\text { (Route) }\end{array}$ & System & $\begin{array}{r}\text { NOAE } \\
(p p n\end{array}$ \\
\hline \multicolumn{5}{|c|}{$\begin{array}{l}\text { ACUTE EXPOSURE } \\
\text { Systemic }\end{array}$} \\
\hline \multirow[t]{5}{*}{1} & $\begin{array}{l}\text { Rat } \\
\text { (Fischer- }\end{array}$ & 44) $\begin{array}{l}2 \mathrm{wk} \\
5 \mathrm{~d} / \mathrm{wk} \\
6 \mathrm{hr} / \mathrm{d}\end{array}$ & Resp & 25 \\
\hline & & & Hemato & 25 \\
\hline & & & Hepatic & 25 \\
\hline & & & Renal & 25 \\
\hline & & & $\mathrm{Bd} \mathrm{Wt}$ & 25 \\
\hline
\end{tabular}

$2 \quad$ Mouse $5 \mathrm{~min}$

(Swiss OF1)

Immunol Lymphoret

3 Rat $2 \mathrm{wk}$

(Fischer- 344) $5 \mathrm{~d} / \mathrm{wk}$

$4 \quad$ Mouse $5 \mathrm{~d}$

(CD-1) $\quad 3 \mathrm{hr} / \mathrm{d}$

\section{Neurological}

5 Rat

Harlan-

Wistar
$234 \mathrm{~F}$
De Ceaurriz et al. 1981

respiration rate)

Hoffman et al. 2001

Aranyi et al. 1986

Flickinger 1976
NOAELs are for organ weights and histopathology.

NOAEL is for

histopathology of the spleen.

NOAEL is for no change in susceptibility to infectious agents.

NOAEL is for no adverse neurological signs. 
Table 3-1 Levels of Significant Exposure to Phenol - Inhalation

(continued)

\begin{tabular}{llr}
\hline & & $\begin{array}{r}\text { Exposu } \\
\text { Duration }\end{array}$ \\
$\begin{array}{l}\text { Key to } \\
\text { Figure }\end{array}$ & $\begin{array}{r}\text { Species } \\
\text { (Strain) } \\
\text { Frequen } \\
\text { (Route) }\end{array}$ \\
\hline 6 & $\begin{array}{l}\text { Rat } \\
\text { (Harlan- } \\
\text { Wistar) }\end{array}$ & \\
& $8 \mathrm{hr}$ \\
\hline
\end{tabular}

INTERMEDIATE EXPOSURE

Death

$\begin{array}{lll}7 & \text { Gn Pig } & 6 \mathrm{wk} \\ & \text { (NS) } & 5 \mathrm{~d} / \mathrm{wk}\end{array}$$$
7 \mathrm{hr} / \mathrm{d}
$$

Systemic

$8 \quad$ Monkey 90

(Rhesus) $\quad 24 \mathrm{hr} / \mathrm{d}$

$\begin{array}{ll}\text { Resp } & 5 \mathrm{M} \\ \text { Hemato } & 5 \mathrm{M} \\ \text { Hepatic } & 5 \mathrm{M} \\ \text { Renal } & 5 \mathrm{M} \\ \text { Bd Wt } & 5 \mathrm{M}\end{array}$

9 Rat

$\begin{array}{ll}\text { Rat } & 15 \mathrm{~d} \\ \text { (White) } & 24 \mathrm{hr} / \mathrm{d}\end{array}$

$24 \mathrm{hr} / \mathrm{d}$

Hemato

26

Hepatic

$\mathrm{Bd} \mathrm{Wt}$
$234 \mathrm{~F}$ (loss of coordination and Flickinger 1976 tremors)

(5/12 deaths) Deichmann et al. 1944

U.S. Air Force 1961

NOAELs are for organ histopathology.

26 (serum activities of ALT,

AST, LDH, and GLDH

increased 2-6-fold;

increased serum

magnesium) 
Table 3-1 Levels of Significant Exposure to Phenol - Inhalation

(continued)

\begin{tabular}{|c|c|c|c|c|}
\hline $\begin{array}{l}\text { Key to } \\
\text { Figure }\end{array}$ & $\begin{array}{l}\text { Species } \\
\text { (Strain) }\end{array}$ & $\begin{array}{c}\text { Exposurel } \\
\text { Duration/ } \\
\text { Frequency } \\
\text { (Route) }\end{array}$ & System & $\begin{array}{r}\text { NOAE } \\
\text { (ppn }\end{array}$ \\
\hline \multirow[t]{4}{*}{10} & $\begin{array}{l}\text { Rat } \\
\text { (NS) }\end{array}$ & $\begin{array}{l}10 \mathrm{wk} \\
5 \mathrm{~d} / \mathrm{wk} \\
7 \mathrm{hr} / \mathrm{d}\end{array}$ & Resp & 26 \\
\hline & & & Cardio & 26 \\
\hline & & & Hepatic & 26 \\
\hline & & & Renal & 26 \\
\hline
\end{tabular}

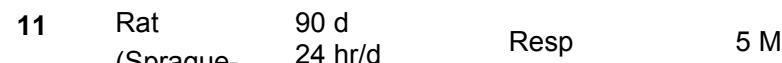

(Sprague- $24 \mathrm{hr} / \mathrm{d}$
Dawley)

LOAEL

OAEL

Reference

(ppm)
(perious

Chemical Form

Comments

$\begin{array}{ll}\text { Hemato } & 5 \mathrm{M} \\ \text { Hepatic } & 5 \mathrm{M} \\ \text { Renal } & 5 \mathrm{M} \\ \text { Bd Wt } & 5 \mathrm{M}\end{array}$

Mouse $\quad 90 \mathrm{~d} \quad$ Resp

(NS) $\quad 24 \mathrm{hr} / \mathrm{d}$

Resp

$5 \mathrm{M}$

Deichmann et al. 1944

NOAELs are for

histopathology of

tissues.

$\begin{array}{ll} & \\ \text { Hemato } & 5 \mathrm{M} \\ \text { Hepatic } & 5 \mathrm{M} \\ \text { Renal } & 5 \mathrm{M} \\ \text { Bd Wt } & 5 \mathrm{M}\end{array}$

U.S. Air Force 1961

U.S. Air Force 1961
NOAELs are for histopathology of tissues. 
Table 3-1 Levels of Significant Exposure to Phenol - Inhalation

\begin{tabular}{|c|c|c|}
\hline $\begin{array}{l}\text { Key to } \\
\text { Figure }\end{array}$ & $\begin{array}{l}\text { Species } \\
\text { (Strain) }\end{array}$ & $\begin{array}{l}\text { Exposurel } \\
\text { Duration/ } \\
\text { Frequency } \\
\text { (Route) }\end{array}$ \\
\hline 13 & $\begin{array}{l}\text { Gn Pig } \\
\text { (NS) }\end{array}$ & $\begin{array}{l}6 \mathrm{wk} \\
5 \mathrm{~d} / \mathrm{wk} \\
7 \mathrm{hr} / \mathrm{d}\end{array}$ \\
\hline
\end{tabular}

LOAEL

\begin{tabular}{cccccc} 
& NOAEL & Less Serious & Serious & Reference \\
System & $(\mathrm{ppm})$ & $(\mathrm{ppm})$ & $(\mathrm{ppm})$ & Chemical Form & Comments \\
\hline
\end{tabular}

Cardio

Hepatic

Renal
26 (acute lobular pneumonia Deichmann et al. 1944 with occasional

abscesses and vascular

damage)

26 (necrosis of the

myocardium, extensive

reactive inflammation)

26 (fatty changes,

centrolobular

degeneration and

necrosis)

26 (edema of the convoluted

tubules, slightly

advanced focal cortical

lesions, glomerular

degeneration) 
Table 3-1 Levels of Significant Exposure to Phenol - Inhalation

\begin{tabular}{|c|c|c|}
\hline $\begin{array}{l}\text { Key to } \\
\text { Figure }\end{array}$ & $\begin{array}{c}\text { Species } \\
\text { (Strain) }\end{array}$ & $\begin{array}{l}\text { Exposurel } \\
\text { Duration/ } \\
\text { Frequency } \\
\text { (Route) }\end{array}$ \\
\hline 14 & $\begin{array}{l}\text { Rabbit } \\
\text { (NS) }\end{array}$ & $\begin{array}{l}12 \mathrm{wk} \\
5 \mathrm{~d} / \mathrm{wk} \\
7 \mathrm{hr} / \mathrm{d}\end{array}$ \\
\hline
\end{tabular}

LOAEL

\begin{tabular}{|c|c|c|c|c|c|}
\hline \multirow[b]{2}{*}{ ystem } & \multicolumn{5}{|c|}{ LOAEL } \\
\hline & $\begin{array}{r}\text { NOAEL } \\
(p p m)\end{array}$ & $\begin{array}{c}\text { Less Serious } \\
\text { (ppm) }\end{array}$ & & $\begin{array}{l}\text { ious } \\
\text { ppm) }\end{array}$ & $\begin{array}{l}\text { Reference } \\
\text { Chemical Form }\end{array}$ \\
\hline sp & & & 26 & $\begin{array}{l}\text { (confluent lobular } \\
\text { pneumonia, chronic } \\
\text { purulent bronchitis, } \\
\text { hyperplastic peribronchial } \\
\text { tissue, degenerative } \\
\text { changes in pulmonary } \\
\text { vessels) }\end{array}$ & Deichmann et al. 1944 \\
\hline rdio & & & 26 & $\begin{array}{l}\text { (myocardial } \\
\text { degeneration, necrosis of } \\
\text { muscle bundles, } \\
\text { interstitial fibrosis, } \\
\text { lymphocytic infiltration) }\end{array}$ & \\
\hline patic & & & 26 & $\begin{array}{l}\text { (centrilobular } \\
\text { degeneration and } \\
\text { necrosis) }\end{array}$ & \\
\hline nal & & & 26 & $\begin{array}{l}\text { (edema of the convoluted } \\
\text { tubules, focal cortical } \\
\text { lesions, glomerular } \\
\text { degeneration) }\end{array}$ & \\
\hline
\end{tabular}

Neurological

15 Monkey $\quad 90 \mathrm{~d}$

(Rhesus) $\quad 24 \mathrm{hr} / \mathrm{d}$

16

$\begin{array}{ll}\text { Rat } & 15 \mathrm{~d} \\ \text { (White) } & 24 \mathrm{hr} / \mathrm{d}\end{array}$

26 (mild motor disorders during the first 4 days of exposure, 4.4 degrees decrease in sliding angle)
U.S. Air Force 1961

Dalin and Kristoffersson 1974 
Table 3-1 Levels of Significant Exposure to Phenol - Inhalation

(continued)

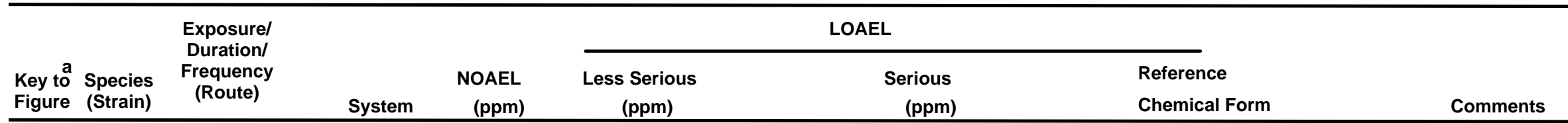

17 Rat $90 \mathrm{~d}$

(Sprague- $24 \mathrm{hr} / \mathrm{d}$

Dawley)

18

$\begin{array}{ll}\text { Mouse } & 90 \mathrm{~d} \\ \text { (NS) } & 24 \mathrm{hr} / \mathrm{d}\end{array}$

19

$\begin{array}{ll}\text { Gn Pig } & 6 \mathrm{wk} \\ \text { (NS) } & 5 \mathrm{~d} / \mathrm{wk} \\ & 7 \mathrm{hr} / \mathrm{d}\end{array}$

$5 \mathrm{M}$

$5 \mathrm{M}$
U.S. Air Force 1961

U.S. Air Force 1961

Deichmann et al. 1944

NOAEL is for brain histopathology and results of a swimming test.

NOAEL is for brain histopathology and results of a swimming

test.

a The number corresponds to entries in Figure 3-1.

$\mathrm{ALT}=$ alanine aminotransferase; $\mathrm{AST}=$ aspartate aminotransferase; $\mathrm{Bd} \mathrm{Wt}=$ body weight $;$ Cardio $=$ cardiovascular $; \mathrm{d}=$ day $(\mathrm{s}) ;$ Endocr $=$ endocrine; $\mathrm{F}=\mathrm{Female} ; \mathrm{Gastro}=$

gastrointestinal; GLDH = glutamate dehydrogenase; $\mathrm{Gn}$ pig = guinea pig; Hemato = hematological; hr = hour(s); Immuno = immunological; LDH = lactate dehydrogenase; LOAEL =

owest-observed-adverse-effect level; $\mathrm{M}=$ male; $\mathrm{min}=$ minute(s); NOAEL = no-observed-adverse-effect level; NS = not specified; Resp = respiratory; wk = week(s); yr = year(s) 
Figure 3-1 Levels of Significant Exposure to Phenol - Inhalation

Acute $(\leq 14$ days)

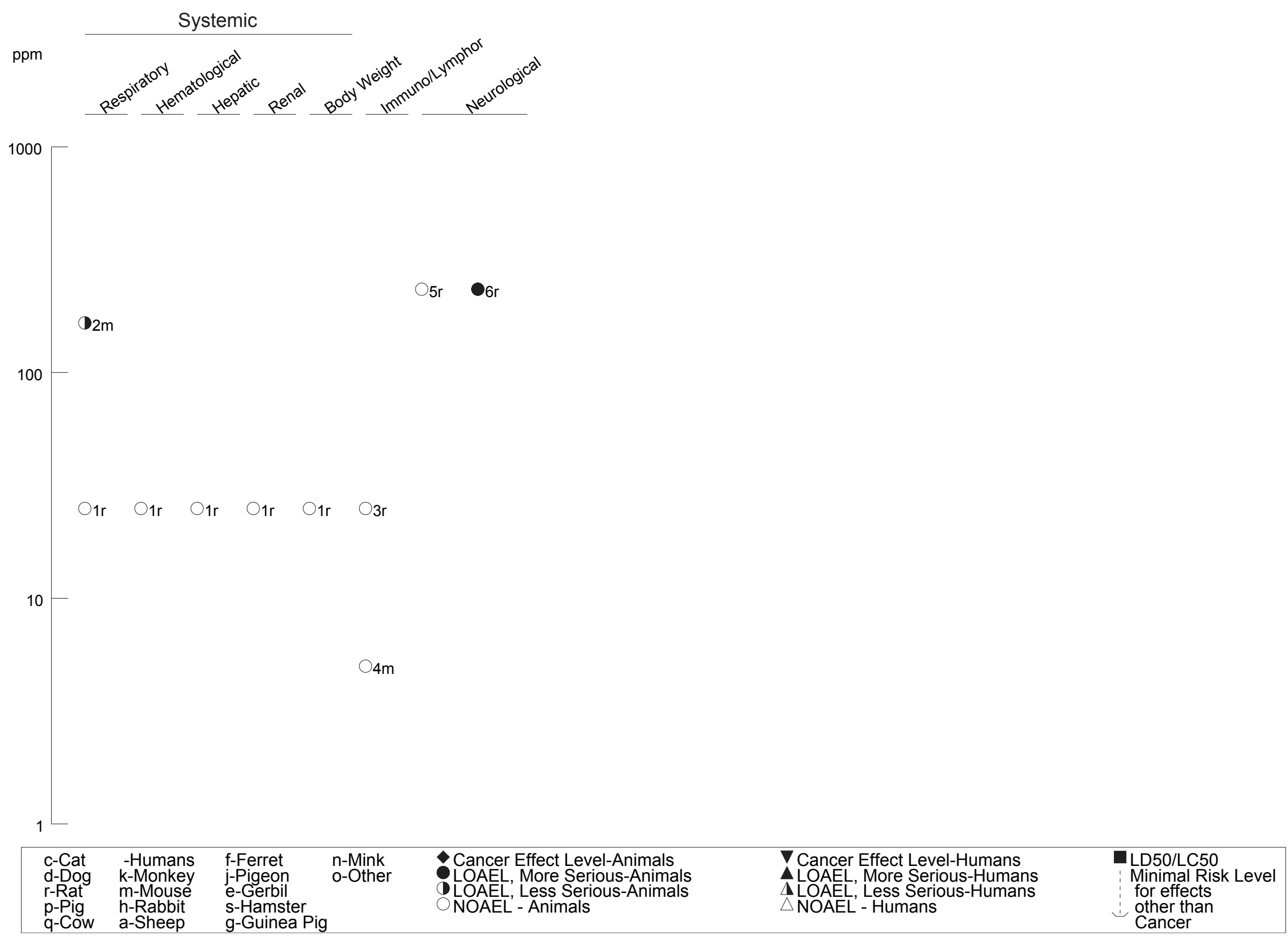


Figure 3-1 Levels of Significant Exposure to Phenol - Inhalation (Continued)

Intermediate (15-364 days)

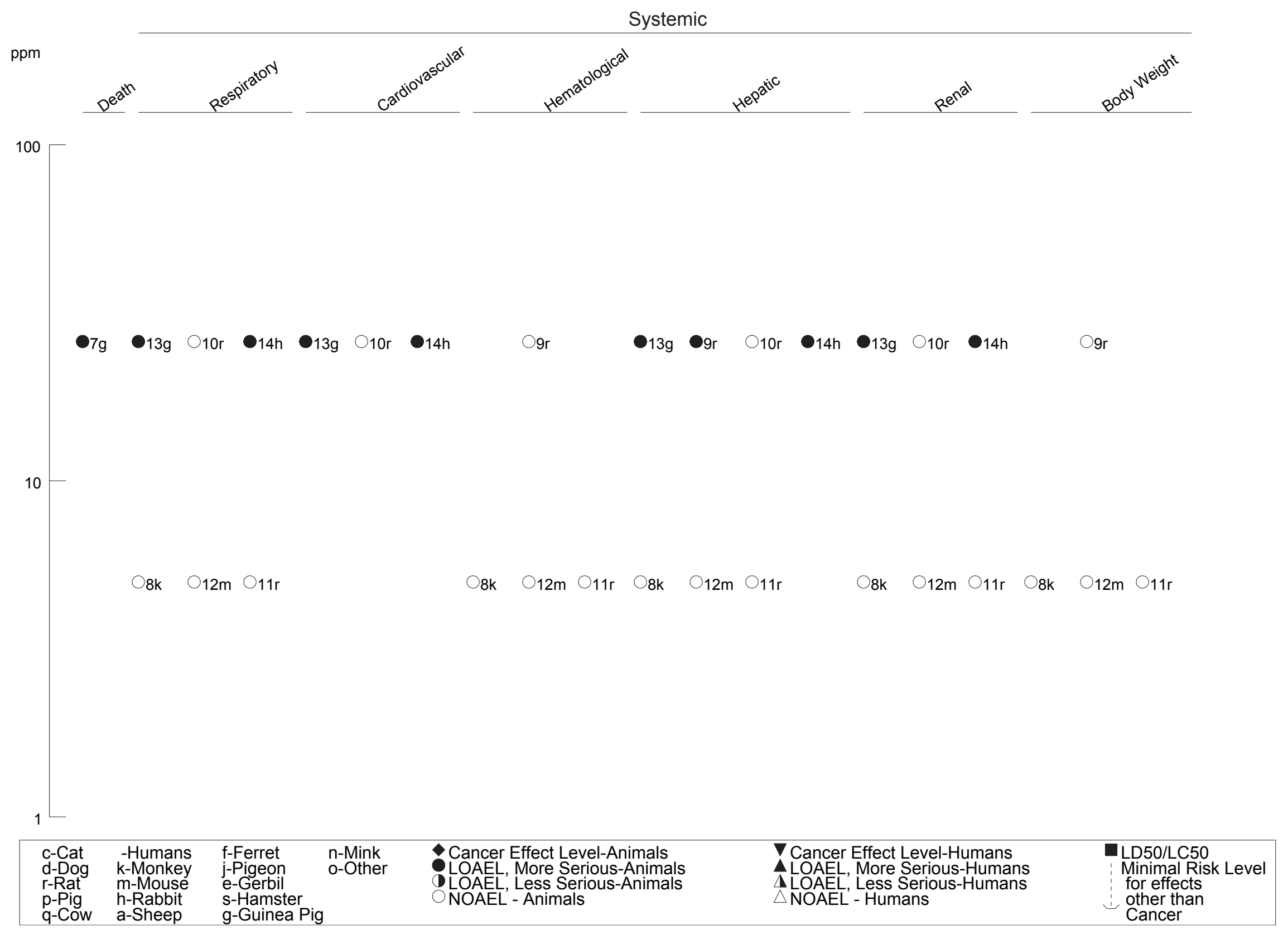


Figure 3-1 Levels of Significant Exposure to Phenol - Inhalation (Continued)

Intermediate (15-364 days)

ppm

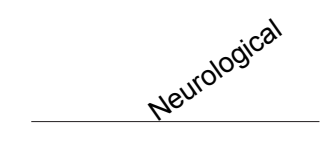

100

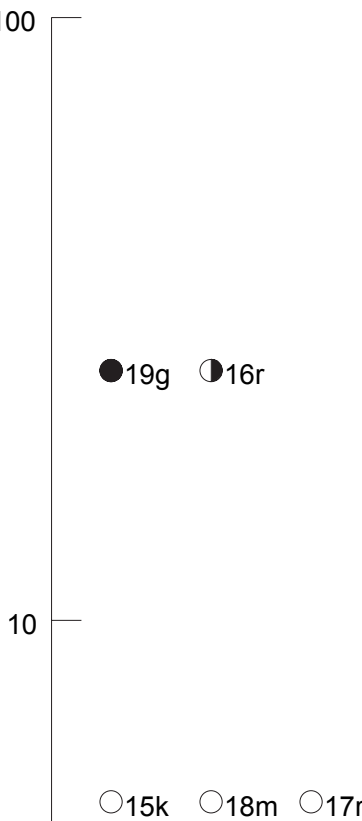

$1 \longleftarrow$

\begin{tabular}{|c|c|c|c|c|c|c|}
\hline $\begin{array}{l}\text { c-Cat } \\
\text { d-Dog } \\
\text { r-Rat } \\
\text { p-Pig } \\
\text { q-Cow }\end{array}$ & $\begin{array}{l}\text {-Humans } \\
\text { k-Monkey } \\
\text { m-Mouse } \\
\text { h-Rabbit } \\
\text { a-Sheep }\end{array}$ & $\begin{array}{l}\text { f-Ferret } \\
\text { j-Pigeon } \\
\text { e-Gerbil } \\
\text { s-Hamster } \\
\text { g-Guinea Pig }\end{array}$ & $\begin{array}{l}\text { n-Mink } \\
\text { o-Other }\end{array}$ & $\begin{array}{l}\text { Cancer Effect Level-Animals } \\
\text { LOAEL, More Serious-Animals } \\
\text { LOAEL, Less Serious-Animals } \\
\text { NOAEL - Animals }\end{array}$ & $\begin{array}{l}\nabla \text { Cancer Effect Level-Humans } \\
\Delta \text { LOAEL, More Serious-Humans } \\
\Lambda \text { LOAEL, Less Serious-Humans } \\
\triangle \text { NOAEL - Humans }\end{array}$ & $\begin{array}{l}\text { LD50/LC50 } \\
\text { Minimal Risk Level } \\
\text { for effects } \\
\text { other than } \\
\text { Cancer }\end{array}$ \\
\hline
\end{tabular}
g-Guinea Pig

Cancer 
Ksylamit ${ }^{\mathrm{TM}}$ is indicated to consist of “.... mixture of chlorinated benzenes, pentachlorophenol, alpha-chloronaphthalene, chloroparaffin and kerosene...", and that the authors provide no discussion of how phenol and formaldehyde are produced through the use of such a mixture. Twenty-two workers (18 women and 4 men) exposed for at least 6 months were the cases, and 29 non-exposed, nonsmoking volunteers matched for age, sex, and place of residence were the controls. The investigators indicate that all exposed workers developed chronic complaints, among them cough and sore throat, but that no remarkable increase in morbidity was found during the 6 months of exposure to Ksylamit ${ }^{\mathrm{TM}}$ nor during the 3-year follow-up study (details of which were not provided). The investigators attribute these symptoms to the irritant effect of the inhaled Ksylamit ${ }^{\mathrm{TM}}$ probably (based on the references provided) due to the formaldehyde vapor they assert emanates from the wood-preserving liquid.

In laboratory animals, phenol is a respiratory irritant. De Ceaurriz et al. (1981) reported a dose-response function for reflex apnea, an index of respiratory irritation, in mice exposed to phenol vapor. From the $\log$ dose-response function for decreased breathing rate, the $\mathrm{RD}_{50}(\mathrm{RD}$ designates respiratory depression), or level of phenol in air that resulted in a $50 \%$ decrease in breathing rate during a 5 -minute head-only exposure, was established as $166 \mathrm{ppm}$. Based on the $\mathrm{RD}_{50}$, the study authors estimated that a concentration of $17 \mathrm{ppm}\left(0.1 \times \mathrm{RD}_{50}\right)$ would be a LOAEL for respiratory irritation in humans, and a concentration of $2 \mathrm{ppm}\left(0.01 \times \mathrm{RD}_{50}\right)$ would be a NOAEL.

In a study in which female Harlan Wistar rats were exposed for 1 hour to a phenol aerosol at a concentration of $234 \mathrm{ppm}$, then held for 2 weeks postexposure, Flickinger (1976) observed signs of nasal irritation during exposure. However, all animals exhibited normal behavior by postexposure day 1, and no abnormal lesions were observed upon gross autopsy. No histopathology was performed; thus, this study is not presented as a LOAEL for rats. A more recent study in which rats were exposed intermittently nose-only to up to $25 \mathrm{ppm}$ phenol for 2 weeks found no significant gross or microscopic alterations in the respiratory tract, including the nasal turbinates (Hoffman et al. 2001).

Inflammation, cellular infiltration, pneumonia, bronchitis, endothelial hyperplasia, and capillary thrombosis occurred in guinea pigs exposed by inhalation to 26-52 ppm phenol for 41 days (Deichmann et al. 1944). Rabbits exhibited qualitatively similar but less severe effects after 88 days of similar exposure. Rats exposed similarly showed no gross or microscopic alterations in the respiratory tract. Since only a range was given for the exposure level (26-52 ppm), the exact level of phenol in air that resulted in respiratory effects was not established and may be as low as $26 \mathrm{ppm}$ or as high as $52 \mathrm{ppm}$. Interpretation of this study is further complicated by an apparent lack of controls. However, the lung 
pathology was so severe, particularly in the guinea pigs, that it is difficult to ascribe the effects to any source other than the phenol exposure. The lower limit of the exposure range, $26 \mathrm{ppm}$, can be considered a LOAEL for respiratory effects in guinea pigs and rabbits and a NOAEL for rats.

No significant histological abnormalities of the lungs were detected in Rhesus monkeys, rats, or mice exposed to 5 ppm phenol continuously for 90 days (U.S. Air Force 1961).

Cardiovascular Effects. In a cohort mortality study of workers in a large rubber and tire manufacturing plant, Wilcosky and Tyroler (1983) found a significant increase in mortality from ischemic heart disease in phenol exposed workers. Of the 25 solvents used in the plant, phenol exposure showed the strongest association with mortality from heart disease, greater even than that observed for exposure to carbon disulfide, the only known occupational cause of atherosclerosis.

In a cohort-mortality study of workers from five phenol-formaldehyde resin plants, Dosemeci et al. (1991) found a slight reduction in mortality due to heart disease. These investigators hypothesized a protective effect of phenol exposures; however, these results clearly conflict with those of Wilcosky and Tyroler (1983). As a consequence, without more definitive studies, it is difficult to assess the cardiovascular risk to humans, if any, posed by occupational exposure to phenol.

Myocardial injury was reported in guinea pigs exposed to 26-52 ppm for 41 days, rabbits exhibited qualitatively similar but less severe effects after 88 days of similar exposure, and rats showed no significant alterations after 54 exposures (Deichmann et al. 1944). The injury was characterized by myocardial inflammation, degeneration, and necrosis, interstitial fibrosis, and lymphocyte infiltration. Since only a range was given for the exposure level (26-52 ppm), the exact level of phenol in air that resulted in myocardial injury was not established and may be as low as $26 \mathrm{ppm}$ or as high as $52 \mathrm{ppm}$. Interpretation of this study is further complicated by an apparent lack of controls. However, the heart pathology was so severe that it is difficult to ascribe the effects to any source other than the phenol exposure. The lower limit of the exposure range, $26 \mathrm{ppm}$, can be considered a LOAEL for myocardial injury in guinea pigs and rabbits and a NOAEL for rats.

Gastrointestinal Effects. Historical information in a case report (Merliss 1972) indicates that 'carbol marasmus' was a common occupational disorder of physicians and their assistants during the mid19th Century when carbolic acid sprays (1:40 phenol in water) were commonly used for antisepsis in operating rooms. Among the characteristics of this disorder was anorexia leading to progressive weight 
loss and excess production of saliva. Similar gastrointestinal effects were observed in one of the author's patients who was involved in the daily distillation of phenol over a 13.5-year period. Exposed both via inhalation of the vapors and dermally from frequent spills, the patient's symptoms included both loss of appetite and weight loss.

A cohort mortality study of workers in five phenol-formaldehyde resin manufacturing plants found that exposed workers showed a slight reduction in death rate due to cancers of the digestive system as compared to both non-exposed workers and the general population (Dosemeci et al. 1991).

In a study of rats exposed continuously for 15 days to $26 \mathrm{ppm}$ phenol vapor, Dalin and Kristoffersson (1974) noted the absence of alterations in the digestive system and attributed this to the relatively low exposure levels (as compared to studies using oral dosing), but no further discussion was provided.

Hematological Effects. A case-control study of office workers was conducted by Baj et al. (1994) to evaluate the risks of chronic exposures to "inhaled formaldehyde, phenol and isomers of organic chlorohydrocarbons from Ksylamit ${ }^{\mathrm{TM}}$ " which is a widely used liquid wood preservative. It should be noted that in the report, Ksylamit" is indicated to consist of "a mixture of chlorinated benzenes, pentachlorophenol, alpha-chloronaphthalene, chloroparaffin and kerosene," and that the authors provide no discussion of how phenol and formaldehyde are produced through the use of such a mixture. Twenty-two workers (18 women and 4 men) exposed for at least 6 months were the cases, and 29 non-exposed, nonsmoking volunteers matched for age, sex, and place of residence were the controls. Using blood and urine samples drawn after 6 months of exposure, cases and controls were compared on a variety of biochemical, hematological, and immunological parameters. The exposed group showed no differences in any of the blood chemistry parameters examined, serum bilirubin, alanine, and aspartate aminotransferase activity, but had about a $30 \%$ increase in eosinophils, a $25 \%$ increase in monocytes, and a $70 \%$ decrease in erythrocytes. Measurement of the office air at the end of the 6-month period revealed a level of phenol of $0.34 \mathrm{ppm}$. Although the authors contend that their observations support the concern that chronic exposure to phenol could adversely affect the hematopoietic system, it is important to consider not only that other volatile chemicals, chlorinated organics, were present in the wood-preserving liquid, but also that the chemical composition provided for Ksylamit ${ }^{\mathrm{TM}}$ opens up the possibility that the effects being evaluated result from exposure to pentachlorophenol rather than to phenol. This is particularly true since it was not possible to determine from the information presented if the analytical methods used would differentiate between phenol and pentachlorophenol. 
Workers $(n=20)$ at an oil-refining plant in Egypt exposed to a time-weighted mean concentration of $5.4 \mathrm{ppm}$ of phenol in air for a mean exposure period of 13.15 years showed small but significant increases in hemoglobin, hematocrit, mean corpuscular hemoglobin, and mean corpuscular volume, but red blood cell counts were not significantly altered relative to a group of 30 unexposed controls (Shamy et al. 1994). Other small, but significant changes relative to controls included increased basophils and neutrophils, decreased monocytes, and increased clotting time.

Hematocrit and hemoglobin concentrations were not affected in rats exposed to $26 \mathrm{ppm}$ phenol in air continuously for 15 days (Dalin and Kristoffersson 1974). Detailed hematological evaluations including red and white blood cell, reticulocyte, and platelet counts; white cell differential; hemoglobin and sulfhemoglobin, and red cell fragility tests, as well as corpuscular volume, corpuscular hemoglobin, and corpuscular hemoglobin concentrations, did not reveal any effects in Rhesus monkeys, rats, or mice exposed continuously to $5 \mathrm{ppm}$ phenol in air for 90 days (U.S. Air Force 1961). Comprehensive hematology testing of rats exposed nose-only to up to $25 \mathrm{ppm}$ of phenol 6 hours/day, 5 days/week for 2 weeks showed no significant exposure-related deviations from control values (Hoffman et al. 2001).

Musculoskeletal Effects. A case of muscle pain and weakness was described in an individual after intermittent chronic inhalation and dermal exposure to vapors and solutions of phenol, cresol, and xylenol for $>10$ years (Merliss 1972). The symptoms lessened when the subject was removed from exposure. Although the exposure concentrations were not reported, the study author stated that the patient often detected heavy odors, and that phenol was often spilled on his clothes resulting in skin irritation. Since phenol is absorbed readily from the skin, dermal absorption of phenol may have contributed to the systemic effects that were observed. The above symptoms may represent neurological effects rather than injury to the muscle tissue.

Hepatic Effects. Enlarged liver and elevated serum levels of hepatic enzymes indicative of liver injury (lactate dehydrogenase, 2 times above normal; aspartate aminotransferase (AST), 21 times above normal; alanine aminotransferase (ALT), 100 times above normal) were observed in an individual following chronic daily exposure to vapors and spills of phenol for $>10$ years (Merliss 1972). The symptoms lessened when the individual was removed from the site of exposure. Although the exposure concentrations were not reported, the study author stated that the patient often detected heavy odors and that phenol was often spilled on his clothes resulting in skin irritation. Since phenol is absorbed readily from the skin, dermal absorption may have contributed to the systemic effects that were observed. A study of 20 workers at an oil-refining plant in Egypt exposed to a time-weighted average concentration of 
phenol of $5.4 \mathrm{ppm}$ for a mean exposure duration of 13.15 years found small, but significant increases in ALT and AST activities (approximately 65 and 54\%, respectively) in serum collected at the end of the shift of the last working day of the week relative to 30 unexposed controls (Shamy et al. 1994).

No effects on activities of liver enzymes (ALT, AST, $\gamma$-glutamyltranspeptidase, alkaline phosphatase) in the serum or changes in serum bilirubin or ceruloplasmin were noted in 22 workers exposed for 6 months to vapors from a wood-treatment liquid containing phenol, formaldehyde, and organic chlorohydrocarbons (Baj et al. 1994). Although the study authors considered a significant increase in serum iron to reflect an adverse effect on the liver that they attributed to phenol exposure, it is important to consider not only that other volatile chemicals, chlorinated organics, were present in the wood-preserving liquid, but also that the chemical composition provided for Ksylamit ${ }^{\mathrm{TM}}$ opens up the possibility that the effects being evaluated result from exposure to pentachlorophenol rather than phenol. Dosemeci et al. (1991) saw a dose-related decrease in mortality from liver cirrhosis in a cohort of workers occupationally exposed to phenol during their employment at five phenol-formaldehyde resin plants. These findings are complicated by the fact that workers were also exposed to other chemicals; however, the authors hypothesize that exposure to phenol could have a protective effect for diseases involving free radical damage.

Centrilobular degeneration and necrosis of the liver were reported in guinea pigs exposed intermittently by inhalation to 26-52 ppm phenol for 41 days, rabbits exhibited qualitatively similar but less severe effects after 88 days of similar exposure, whereas rats showed no significant alterations after 54 exposures (Deichmann et al. 1944). Since only a range was given for the exposure level (26-52 ppm), the exact level of phenol in air that resulted in hepatic injury was not established and may be as low as $26 \mathrm{ppm}$ or as high as $52 \mathrm{ppm}$. Interpretation of this study is further complicated by an apparent lack of controls. However, the liver pathology was so severe, particularly in the guinea pigs, that it is difficult to ascribe the effects to any source other than the phenol exposure.

Elevated activities of liver enzymes (lactate dehydrogenase, AST, ALT, glutamate dehydrogenase) were found in the serum of rats exposed continuously to $26 \mathrm{ppm}$ phenol vapor for 15 days (Dalin and Kristoffersson 1974). Increased concentration of these enzymes in serum is often associated with liver injury, but is not conclusive evidence for the type or severity of injury. Therefore, $26 \mathrm{ppm}$ can be considered a less serious LOAEL in rats. Serum levels of magnesium were also increased in these rats, an effect the study authors suggested may also be a sign of liver injury. In a more recent study, rats exposed nose-only to up to $25 \mathrm{ppm}$ of phenol 6 hours/day, 5 days/week for 2 weeks showed no evidence of liver 
effects as monitored by clinical chemistry tests and histopathological examination of the liver (Hoffman et al. 2001). No significant histological abnormalities were detected in the livers of Rhesus monkeys, rats, or mice exposed continuously to 5 ppm phenol in air for 90 days (U.S. Air Force 1961).

Renal Effects. In a case of chronic phenol poisoning, dark urine and glucose in the urine were noted in a man following intermittent exposure to vapors and solutions of phenol (Merliss 1972). The urine tested negative for protein and urobilinogen. The urine cleared 2-3 months after removal from exposure. Although the exposure concentrations were not reported, the study author stated that heavy odors were often detectable, and that phenol was often spilled on the patient's clothes resulting in skin irritation. Since phenol is absorbed readily from the skin, dermal absorption may have contributed to the systemic effects that were observed.

Renal proximal tubule and glomerular injury was reported in guinea pigs exposed intermittently by inhalation to 26-52 ppm phenol for 41 days, and rabbits exhibited qualitatively similar but less severe effects after 88 days of similar exposure; rats exposed similarly for 54 days showed no significant effects (Deichmann et al. 1944). Since only a range was given for the exposure level (26-52 ppm), the exact level of phenol in air that resulted in renal injury was not established and may be as low as 26 ppm or as high as $52 \mathrm{ppm}$. Interpretation of this study is further complicated by an apparent lack of controls. However, the kidney pathology was so severe, particularly in the guinea pigs, that it is difficult to ascribe the effects to any source other than the phenol exposure. The lower limit of the exposure range, $26 \mathrm{ppm}$, can be considered a LOAEL for renal injury in guinea pigs and rabbits. No significant histological abnormalities were detected in the kidneys of Rhesus monkeys, rats, or mice exposed continuously to 5 ppm phenol in air for 90 days (U.S. Air Force 1961). No kidney pathology was reported in a study in rats exposed intermittently nose-only to up to 25 ppm of phenol for 2 weeks (Hoffman et al. 2001); specific end points monitored included kidney histopathology, blood urea nitrogen (BUN), and serum creatinine and electrolytes.

Dermal Effects. Historical information in a case report (Merliss 1972) indicates that 'carbol marasmus' was a common occupational disorder of physicians and their assistants during the mid-19th Century. Among the characteristics of this disorder was an odd form of pigmentation, which commonly occurred in the urine, but also occasionally colored the sclera of the eyes, the skin over the nose, and the cheek bones. NIOSH (1983) conducted a survey in an Oregon hospital in response to concerns about respiratory problems and contact dermatitis in housekeeping staff members who were exposed frequently to germicidal solutions containing phenol and other solvents (formaldehyde, cellosolve, ethanolamine). 
According to the survey, the housekeeping staff reported significantly more symptoms of cough, itching, sinus problems, and dermatitis than other employees. Air concentrations of phenol in the work areas were below the limit of detection $(<0.01 \mathrm{ppm})$. Urinary phenol levels in housekeeping staff were not significantly different from those of other employees. Thus, while it is likely that the employees came into contact with irritants, the cause of the reported symptoms could not be assigned to phenol or any other specific substance in the work environment.

No studies were located regarding dermal effects in animals following inhalation exposure to phenol.

Ocular Effects. A case-control study of office workers was conducted by Baj et al. (1994) to evaluate the risks of chronic exposures to "inhaled formaldehyde, phenol and isomers of organic chlorohydrocarbons from Ksylamit ${ }^{\mathrm{TM}}$ " which is a widely used liquid wood preservative reported to consist of "a mixture of chlorinated benzenes, pentachlorophenol, alpha-chloronaphthalene, chloroparaffin, and kerosene." Twenty-two workers (18 women and 4 men) exposed for at least 6 months were the cases, and 29 non-exposed, nonsmoking volunteers matched for age, sex, and place of residence were the controls. The authors indicate that all of the exposed workers developed chronic complaints, among them burning eyes, but that no remarkable increase in morbidity was found during the 6 months of exposure to Ksylamit ${ }^{\mathrm{TM}}$, nor during the 3-year follow-up study (details of which were not provided). The authors attribute these symptoms to the irritant effect of the inhaled Ksylamit ${ }^{\mathrm{TM}}$ probably (based on the references provided) due to the formaldehyde vapor they assert emanates from the wood-preserving liquid.

In a study in which female Harlan Wistar rats were exposed for 1 hour to a phenol aerosol at a concentration of $234 \mathrm{ppm}$, then held for 2 weeks postexposure, Flickinger (1976) observed signs of ocular irritation during exposure. However, all animals were normal by postexposure day 1, and no abnormal lesions were observed upon gross autopsy.

Body Weight Effects. Historical information in a case report (Merliss 1972) indicates that 'carbol marasmus' was a common occupational disorder of physicians and their assistants during the mid-19th Century. Among the characteristics of this disorder were anorexia accompanied by progressive weight loss. The author reported that his patient, a 44-year-old man involved in the daily distillation of phenol, showed many of the symptoms of this condition, including lack of appetite and severe weight loss, probably due to his daily workplace exposures to phenol vapors. Although the exposure concentrations were not reported, the report indicated that the patient often detected heavy odors, and that phenol was 
often spilled on his clothes resulting in skin irritation. Since phenol is absorbed readily from the skin, dermal absorption may have contributed to the systemic effects that were observed.

Body weight effects were not observed in adult female Harlan Wistar rats exposed to an aerosol containing $234 \mathrm{ppm}$ phenol for 8 hours (Flickinger 1976), in rats exposed continuously to $26 \mathrm{ppm}$ phenol in air for 15 days (Dalin and Kristoffersson 1974), in Rhesus monkeys, rats, or mice exposed continuously to $5 \mathrm{ppm}$ phenol in air for 90 days (U.S. Air Force 1961), or in rats exposed intermittently nose-only for 2 weeks to up to 25 ppm of phenol vapor (Hoffman et al. 2001).

Metabolic Effects. Dalin and Kristoffersson (1974) reported elevated serum concentrations of potassium and magnesium in rats exposed to $26 \mathrm{ppm}$ phenol vapor continuously for 15 days. While not necessarily adverse, this effect may be related to the muscle tremors and neurological effects observed following inhalation exposure to phenol (see Section 3.2.1.4). No further information was located regarding metabolic effects of inhaled phenol in animals.

\subsubsection{Immunological and Lymphoreticular Effects}

A case-control study of office workers was conducted by Baj et al. (1994) to evaluate the risks of chronic exposures to "inhaled formaldehyde, phenol and isomers of organic chlorohydrocarbons from Ksylamit" ${ }^{\text {TM" }}$ which is a widely used liquid wood preservative. Twenty-two workers (18 women and 4 men) exposed for at least 6 months were the cases, and 29 non-exposed, nonsmoking volunteers matched for age, sex, and place of residence were the controls. Using blood samples drawn after 6 months of exposure, cases and controls were compared on a variety of immunological parameters. The exposed group showed significantly decreased $(\mathrm{p}<0.05)$ levels of the CD3+, CD4+, and CD8+ subsets of T-lymphocytes, a significant decrease $(\mathrm{p}<0.001)$ in lymphocyte responsiveness to the mitogen phytohemagglutinin (PHA), a significant decrease $(\mathrm{p}<0.05)$ in natural killer $(\mathrm{NK})$ cell cytotoxicity, and a significant decrease $(\mathrm{p}<0.0001)$ in the mixed lymphocyte response assay. Measurement of the office air at the end of the 6-month period revealed a level of phenol of $0.34 \mathrm{ppm}$. Although the authors contend that their observations support the concern that chronic exposure to phenol could adversely affect the immune system, it is important to consider not only that other volatile chemicals, chlorinated organics, were present in the wood-preserving liquid, but also that the chemical composition provided for Ksylamit $^{\mathrm{TM}}$ opens up the possibility that the effects being evaluated resulted from exposure to pentachlorophenol rather than phenol. This is particularly true since it was not possible to determine from 
the information presented if the analytical methods used would differentiate between phenol and pentachlorophenol.

An increased susceptibility to Streptococcus zooepidemicus aerosol was not observed in mice exposed to $5 \mathrm{ppm}$ phenol for 3 hours, or for 5 daily 3-hour periods (Aranyi et al. 1986). Neither did the phenol exposures affect pulmonary bactericidal activity towards Klebsiella pneumonia. Although tests for vulnerability to infectious agents do not represent a comprehensive evaluation of immunological competence, the 5-ppm level can be considered a NOAEL for this specific immunological effect, and is recorded in Table 3-1 and plotted in Figure 3-1. Gross and microscopic examination of the spleen of rats exposed nose-only to up to $25 \mathrm{ppm}$ phenol intermittently for 2 weeks did not reveal any significant exposure-related alterations (Hoffman et al. 2001). This exposure concentration is listed as a NOAEL for lymphoreticular effects in Table 3-1.

\subsubsection{Neurological Effects}

Historical information in a case report (Merliss 1972) indicates that 'carbol marasmus' was a common occupational disorder of physicians and their assistants during the mid-19th Century. Among the characteristics of this disorder were anorexia, headache, and vertigo. The author reported that his patient, the subject of the case report, showed many of the symptoms of this condition, although his chief complaints were weakness and muscle pain in his arms and legs, progressive weight loss, and excess production of saliva. The symptoms lessened when the subject was removed from the site of exposure. Although it is possible that these symptoms resulted from injury to the muscle, it is more likely that they represent a neurological effect. No information on exposure concentrations was presented; however, the report indicated that the patient often detected heavy odors and that phenol was often spilled on his clothes resulting in skin irritation. Since phenol is absorbed readily from the skin, dermal absorption may have contributed to the systemic effects that were observed.

A case-control study of office workers was conducted by Baj et al. (1994) to evaluate the risks of chronic exposures to "inhaled formaldehyde, phenol and isomers of organic chlorohydrocarbons from Ksylamit ${ }^{\mathrm{TM}}$ " which is a widely used liquid wood preservative. Twenty-two workers (18 women and 4 men) exposed for at least 6 months were the cases, and 29 non-exposed, nonsmoking volunteers matched for age, sex, and place of residence were the controls. The workers complained of a variety of chronic symptoms, among them headache and fatigue. Measurement of the office air at the end of the 6 month period revealed a level of phenol of $0.34 \mathrm{ppm}$. Although these symptoms could be a sign that 
chronic inhalation exposure to phenol could adversely affect the neurological system, it is important to consider not only that other volatile chemicals, chlorinated organics, were present in the wood-preserving liquid, but also that the chemical composition provided for Ksylamit ${ }^{\mathrm{TM}}$ opens up the possibility that the effects being evaluated resulted from exposure to pentachlorophenol rather than phenol. This is particularly true since it was not possible to determine from the information presented if the analytical methods used would differentiate between phenol and pentachlorophenol.

Female Harlan Wistar rats exposed for 1 or 8 hours to $234 \mathrm{ppm}$ phenol delivered in an aerosol demonstrated no neurological effects at 1 hour, a slight loss of coordination with spasm of the muscle groups at 4 hours, and frank tremors leading to a severe loss of coordination by 8 hours (Flickinger 1976). All animals were normal by postexposure day 1, and no abnormal lesions were observed upon gross autopsy performed at the end of a 14-day observation period. These exposure levels are recorded in Table 3-1 and are plotted in Figure 3-1 as a NOAEL, a less serious LOAEL, and a serious LOAEL.

Rats exposed continuously to 26 ppm showed numerous symptoms and signs of neurological impairment, including muscle tremors, twitching, and disturbances in walking rhythm and posture during the first 35 days of exposure, and impaired performance ( $4.4^{\circ}$ decrease in sliding angle) on a tilting plane test after 15 days of exposure (Dalin and Kristoffersson 1974). These effects are indicative of neurological impairment. Because the tremors did not last during the whole exposure period, the effects were not considered severe.

Hindlimb paralysis was reported in guinea pigs exposed to 26-52 ppm phenol for 41 days (Deichmann et al. 1944). Rabbits and rats exhibited no overt neurological effects after 88 and 74 days of similar exposure, respectively. Since only a range was given for the exposure level (26-52 ppm), the exact level of phenol in air that resulted in hindlimb paralysis was not established and may be as low as 26 ppm or as high as $52 \mathrm{ppm}$. Interpretation of this study is further complicated by an apparent lack of controls. However, the neurological effect was so severe in the guinea pigs that it is difficult to ascribe the effects to any source other than the phenol exposure. The lower limit of the exposure range, $26 \mathrm{ppm}$, is recorded in Table 3-1 and plotted in Figure 3-1 as a LOAEL for serious neurological effects in guinea pigs. Since the presence or absence of overt neurological effects such as paralysis is not a sensitive end point for detecting neurological effects, $26-52 \mathrm{ppm}$ is not considered a reliable NOAEL for neurological effects in rats and rabbits. 
There are several differences in the experimental designs of the Dalin and Kristoffersson (1974) and Deichmann et al. (1944) studies that may account for the different results regarding neurological effects in rats. Dalin and Kristoffersson (1974) reported subtle effects that may have been overlooked in the Deichmann et al. (1944) study. Furthermore, Dalin and Kristoffersson (1974) subjected the rats to a specific test for neurological impairment, the tilting plane test. Although exposure concentrations were the same in both studies, Dalin and Kristoffersson (1974) exposed rats continuously, while Deichmann et al. (1944) exposed rats intermittently. Because phenol is metabolized quite rapidly (see Section 3.4.3), rats exposed intermittently may not develop neurological effects.

Histopathological changes in the brain were not observed in Rhesus monkeys, rats, or mice exposed continuously to $5 \mathrm{ppm}$ phenol in air for 90 days (U.S. Air Force 1961).

The highest NOAEL values and all LOAEL values from each reliable study for neurological effects in each species for acute and intermediate-duration exposure are recorded in Table 3-1 and plotted in Figure 3-1.

\subsubsection{Reproductive Effects}

The only relevant information located is that from a retrospective study of pregnancy outcome among university laboratory employees in Sweden (Axelsson et al. 1984). No significant increase in the rate of miscarriage was found in a group of 576 women exposed to organic solvent relative to 576 unexposed pregnancies. Specific mention of phenol was reported in only five cases, all of which were normal deliveries. No relevant information was found in animal inhalation studies.

\subsubsection{Developmental Effects}

Neither perinatal death rates nor prevalence of malformations were significantly increased in the study of laboratory workers conducted by Axelsson et al. (1984) mentioned above. An abstract by Hernberg et al. (1983) on data from personal interviews of 1,047 Finnish mothers exposed to disinfectants (including phenol) during early pregnancy did not indicate significant associations between exposure to disinfectants and the occurrence of congenital defects. No further relevant information in humans was located. No developmental inhalation studies in animals were located. 


\subsubsection{Cancer}

In a nested case-control study of cancers associated with chemical exposures in the wood industry, Kauppinen et al. (1986) found a significantly increased risk of respiratory system cancer associated with exposure to phenol and phenol in wood dust. As is often the case in occupational settings, these exposures were confounded by smoking and exposures to other materials like pesticides; in addition, information on direct phenol measurements was not provided. The increased risk observed for exposure to phenol was almost 5-fold (odds ratio of 4.94), but showed no dose-related increase. This risk dropped to 4-fold with adjustments for smoking history, and $<3$-fold (and non-significant) when workers exposed to both phenols and pesticides were excluded from the analysis.

Similar to the findings of Kauppinen et al. (1986), a large $(14,861)$ cohort mortality study of workers in the phenol-formaldehyde resin manufacturing industry found nondose-related increases in the risk of several respiratory system cancers in workers exposed to phenol (Dosemeci et al. 1991). The authors develop a semiquantitative exposure assessment by assigning exposure levels (none, low, medium, and high) to each job category. The increased risks were small; for instance, for cancer of the larynx or lung, standard mortality ratios (SMRs) of 1.1 were less than those found for non-exposed workers. For a number of other cancers, including those of the esophagus, rectum, bladder, kidney, and Hodgkin's disease, the SMRs found for phenol-exposed workers were slightly elevated, but none of the increases were statistically significant when compared with those in the general population. Furthermore, none of these increases had dose-response relationships with cumulative exposure to phenol.

No studies were located regarding cancer in animals following inhalation exposure to phenol.

\subsubsection{Oral Exposure}

\subsubsection{Death}

There have been numerous reports of suicide or suicide attempts involving ingestion of large amounts of phenol. However, the lack of accurate documentation of dose levels in these cases makes it difficult to identify a minimal dose at which lethality occurs. Deichmann and Keplinger (1981), in summarizing the literature, indicated that an oral dose as low as $1 \mathrm{~g}$ could be fatal in humans, but that occasionally patients had survived doses as high as $65 \mathrm{~g}$. Assuming that these patients were male with an average weight of $70 \mathrm{~kg}$, the lower limit on the dose for death would be $14 \mathrm{mg} / \mathrm{kg}$ and the upper limit would be 
approximately $930 \mathrm{mg} / \mathrm{kg}$. In a review of the toxicology of phenol, Bruce et al. (1987) summarized human oral lethality data from numerous case reports and estimated $140 \mathrm{mg} / \mathrm{kg}$ to be the minimal dose at which death occurs. Stajduhar-Caric (1968) reported a case in which a woman ingested $\approx 10-20 \mathrm{~g}$ of phenol and died within hours. The lower limit of the ingested dose was converted to $172 \mathrm{mg} / \mathrm{kg}$, assuming a $58 \mathrm{~kg}$ body weight, to derive a dose for death, which is recorded in Table 3-2 and plotted in Figure 3-2. Boatto et al. (2004) described the case of a male who ingested a solution containing phenol and cresol and died approximately 30 minutes after ingestion. Toxicology tests revealed that the stomach content, blood, and urine of the individual contained $115.0,58.3$, and $3.3 \mu \mathrm{g} / \mathrm{mL}$ of phenol, respectively. Similar blood concentrations $(56-130 \mu \mathrm{g} / \mathrm{mL})$ were measured in fatal cases reported by Tanaka et al. (1998), Soares and Tift (1982), and Lo Dico et al. (1989).

The oral $\mathrm{LD}_{50}$ has been determined in rats treated by gavage with phenol in water; the $\mathrm{LD}_{50}$ was found to decrease with increasing concentration of phenol in the gavage fluid. The reported $\mathrm{LD}_{50}$ values were $340 \mathrm{mg} / \mathrm{kg}$ in rats gavaged with a solution of $200,000 \mathrm{ppm}$ phenol and $530 \mathrm{mg} / \mathrm{kg}$ in rats gavaged with a solution of 20,000 ppm phenol (Deichmann and Witherup 1944). After rats were treated by gavage with $600 \mathrm{mg} / \mathrm{kg}$ in a 5\% solution, 9 of 305 -week-old rats, 18 of 20 10-day-old rats, and 12 of 20 adult rats died indicating that the 10-day-old rat is more sensitive to phenol than rats in the other age groups tested (Deichmann and Witherup 1944). In pregnant rats treated on gestation days (GDs) 6-15, 7 of 10 rats died at a dose of $125 \mathrm{mg} / \mathrm{kg} /$ day when treated with a volume of $1 \mathrm{~mL} / \mathrm{kg}$, while 1 of 6 rats died at a dose of $160 \mathrm{mg} / \mathrm{kg} /$ day when treated with a volume of $5 \mathrm{~mL} / \mathrm{kg}$ (NTP 1983a). In a 1-day dosing regimen study, female rats were given $0,12,40,120$, or $224 \mathrm{mg} / \mathrm{kg}$ in order to determine a single-dose oral $\mathrm{LD}_{50}$ of $400 \mathrm{mg} / \mathrm{kg}$ (Berman et al. 1995). Mortality was observed only at the highest dose where two of eight rats treated died. All female rats treated for 14 days with a dose of $120 \mathrm{mg} / \mathrm{kg} / \mathrm{day}$ died (Berman et al. 1995; Moser et al. 1995). In a 14-day dosing regimen with the same doses (except the $224 \mathrm{mg} / \mathrm{kg}$ ), all animals died at the dose of $120 \mathrm{mg} / \mathrm{kg}$.

The oral $\mathrm{LD}_{50}$ of phenol has been estimated as $300 \mathrm{mg} / \mathrm{kg}$ in mice (von Oettingen and Sharpless 1946). Five of 10 rabbits treated with an oral dose of $420 \mathrm{mg} / \mathrm{kg}$ died (Deichmann and Witherup 1944). In pregnant mice treated on GDs 6-15, four of 35 mice died at a dose of $280 \mathrm{mg} / \mathrm{kg} /$ day (NTP 1983b).

Flickinger (1976) gave male Harlan-Wistar albino rats single doses of 0, 200, 398, 795, and 1,580 mg/kg phenol by gavage and held the animals for 14 days postdosing in order to determine an oral $\mathrm{LD}_{50}$. No animals died following the 0,200 , or $398 \mathrm{mg} / \mathrm{kg}$ doses; four of five animals died the first day following 
Table 3-2 Levels of Significant Exposure to Phenol - Oral

\begin{tabular}{|c|c|c|c|c|c|c|c|c|}
\hline \multirow[b]{2}{*}{$\begin{array}{l}\text { Key to } \\
\text { Figure }\end{array}$} & \multirow[b]{2}{*}{$\begin{array}{l}\text { Species } \\
\text { (Strain) }\end{array}$} & \multirow{2}{*}{$\begin{array}{l}\text { Exposurel } \\
\text { Durationl } \\
\text { Frequency } \\
\text { (Route) }\end{array}$} & \multirow[b]{2}{*}{ System } & \multirow[b]{2}{*}{$\begin{array}{l}\text { NOAEL } \\
\text { (mg/kg/day) }\end{array}$} & \multicolumn{2}{|c|}{ LOAEL } & & \multirow[b]{2}{*}{ Comments } \\
\hline & & & & & $\begin{array}{r}\text { Less Serious } \\
\text { (mg/kg/day) }\end{array}$ & $\begin{array}{l}\text { Serious } \\
\text { (mg/kg/day) }\end{array}$ & $\begin{array}{l}\text { Reference } \\
\text { Chemical Form }\end{array}$ & \\
\hline
\end{tabular}

\section{ACUTE EXPOSURE}

Death

1 Human once

$172 \mathrm{~F}$ (death)

Stajduhar-Caric 1968

2 Rat once

(Fischer- 344) (GW)

$$
\begin{array}{ll}
\begin{array}{ll}
\text { Rat } & 14 \mathrm{~d} \\
\text { (Fischer- 344) } & 1 \mathrm{x} / \mathrm{d} \\
& (\mathrm{GW})
\end{array}
\end{array}
$$

$4 \quad$ Rat

Rat once

(Wistar) (GW)

5 Rat $10 d$

(CD) $\quad \begin{aligned} & \text { Gd 6-15 } \\ & 1 \mathrm{x} / \mathrm{d}\end{aligned}$

$1 \mathrm{x} / \mathrm{d}$

6 Mouse $10 d$

(CD-1) Gd 6-15

$\mathrm{x} / \mathrm{d}$

$(\mathrm{GW})$

7 Mouse once

(NS) (GO)

Rabbit once

(White) (GW)

$400 \mathrm{~F}(\mathrm{LD} 50)$

$120 \mathrm{~F}(8 / 8 \mathrm{died})$

$340 \quad($ LD50)

$125 \mathrm{~F}(7 / 10$ maternal deaths $)$

420 (5/10 deaths)
NTP 1983a

Berman et al. 1995

Berman et al. 1995

Dosing volume was 1 $\mathrm{mL} / \mathrm{kg}$.

Deichmann and Witherup 1944

Dosing volume was 1 $\mathrm{mL} / \mathrm{kg}$.

Dosing volume was 10 $\mathrm{mL} / \mathrm{kg}$.
NTP 1983b

$300 \mathrm{M}$ (5/10 deaths)

$280 \mathrm{~F}$ (4/35 maternal deaths)

Deichmann and Witherup 1944 
Table 3-2 Levels of Significant Exposure to Phenol - Oral

(continued)

\begin{tabular}{|c|c|c|c|c|c|c|c|c|}
\hline \multirow[b]{2}{*}{$\begin{array}{l}\text { Key to } \\
\text { Figure }\end{array}$} & \multirow[b]{2}{*}{$\begin{array}{l}\text { Species } \\
\text { (Strain) }\end{array}$} & \multirow{2}{*}{$\begin{array}{l}\text { Exposurel } \\
\text { Durationl } \\
\text { Frequency } \\
\text { (Route) }\end{array}$} & \multirow[b]{2}{*}{ System } & \multirow[b]{2}{*}{$\begin{array}{l}\text { NOAEL } \\
\text { (mg/kg/day) }\end{array}$} & \multicolumn{3}{|c|}{ LOAEL } & \multirow[b]{2}{*}{ Comments } \\
\hline & & & & & $\begin{array}{r}\text { Less Serious } \\
(\mathrm{mg} / \mathrm{kg} / \mathrm{day})\end{array}$ & $\begin{array}{l}\text { Serious } \\
\text { (mg/kg/day) }\end{array}$ & $\begin{array}{l}\text { Reference } \\
\text { Chemical Form }\end{array}$ & \\
\hline
\end{tabular}

\section{Systemic}

9 Rat

Rat once

(Fischer- 344) (GW)

Hepatic $224 \mathrm{~F}$

Renal $\quad 120 \mathrm{~F}$

Endocr $\quad 120 \mathrm{~F} \quad 224 \mathrm{~F}$ (unspecified changes in the adrenal gland)

Bd Wt $\quad 224 \mathrm{~F}$

10 Rat

Rat $14 \mathrm{~d} \quad$ Hepatic $\quad 40 \mathrm{~F}$

(Fischer- 344) $1 \mathrm{x} / \mathrm{d}$

(GW)

Renal

Endocr

$40 \mathrm{~F}$

Bd Wt

$40 \mathrm{~F}$

11

$\begin{array}{ll}\begin{array}{ll}\text { Rat } & 14 d \\ \text { (Fischer- 344) } & \text { Gd 6-19 } \\ & (G W)\end{array} & \text { Resp }\end{array}$

Bd Wt
Berman et al. 1995

Dosing volume was 1 $\mathrm{mL} / \mathrm{kg}$.

(mg/kg/day)

$224 \mathrm{~F}$ (renal tubular necrosis protein casts, papillary hemorrhage)

Berman et al. 1995

Dosing volume was 1 $\mathrm{mL} / \mathrm{kg}$.

$40 \mathrm{~F}$ (renal tubular necrosis, protein casts, papillary hemorrhage in $3 / 8$ )
Narotsky and Kavlock 1995 $\mathrm{mL} / \mathrm{kg}$
$40 \mathrm{~F}(20 \%$ decrease in maternal body weight gain) 
Table 3-2 Levels of Significant Exposure to Phenol - Oral

(continued)

\begin{tabular}{|c|c|c|c|c|c|c|c|c|}
\hline \multirow[b]{2}{*}{$\begin{array}{l}\text { Key to } \\
\text { Figure }\end{array}$} & \multirow[b]{2}{*}{$\begin{array}{l}\text { Species } \\
\text { (Strain) }\end{array}$} & \multirow{2}{*}{$\begin{array}{l}\text { Exposurel } \\
\text { Duration/ } \\
\text { Frequency } \\
\text { (Route) }\end{array}$} & \multirow[b]{2}{*}{ System } & \multirow[b]{2}{*}{$\begin{array}{l}\text { NOAEL } \\
\text { (mg/kg/day) }\end{array}$} & \multicolumn{2}{|c|}{ LOAEL } & \multirow[b]{2}{*}{$\begin{array}{l}\text { Reference } \\
\text { Chemical Form }\end{array}$} & \multirow[b]{2}{*}{ Comments } \\
\hline & & & & & $\begin{array}{l}\text { Less Serious } \\
\text { (mg/kg/day) }\end{array}$ & $\begin{array}{l}\text { Serious } \\
\text { (mg/kg/day) }\end{array}$ & & \\
\hline
\end{tabular}

12 Rat $10 d$

$\begin{array}{llll}\text { Rat } & 10 \mathrm{~d} & \text { Hepatic } & 120 \mathrm{~F} \\ (\mathrm{CD}) & \text { Gd } 6-15 & & \\ & 1 \mathrm{x} / \mathrm{d} & & \\ & (\mathrm{GW}) & & \\ & & \end{array}$

13

$\begin{array}{lll}\text { Rat } & 10 \mathrm{~d} & \mathrm{Bd} \mathrm{Wt} \\ \text { (Sprague- } & \text { Gd 6-15 } & \\ \text { Dawley) } & 3 \times / d & \\ & (\mathrm{GW}) & \end{array}$

14

$\begin{array}{lll}\text { Mouse } & \text { once } & \text { Hemato } \\ \text { (Swiss CD-1) } & \text { Gd 13 } & \end{array}$

(GW)

$\begin{array}{llll}\text { Mouse } & 10 \mathrm{~d} & \text { Hepatic } & 280 \mathrm{~F} \\ \text { (CD-1) } & \text { Gd 6-15 } & & \\ & 1 \times / d & & \\ & (\mathrm{GW}) & & \\ & & \text { Bd Wt } & 140 \mathrm{~F}\end{array}$

Immunol Lymphoret

16 Rat $\quad$ once
$120 \mathrm{~F}$ (11\% reduced maternal body gain on Gd 6-16)
$265 \mathrm{~F}(30-60 \%$ reduction in the ratio of poly/normo
chromatic erythrocytes in
the bone marrow of
pregnant dams)

NTP 1983a

Dosing volume was 5 $\mathrm{mL} / \mathrm{kg}$.

York 1997

osing volume was 10 $\mathrm{mL} / \mathrm{kg}$.

Ciranni et al. 1988

Dosing volume was 10 $\mathrm{mL} / \mathrm{kg}$.

$280 \mathrm{~F}(67 \%$ decrease in

absolute maternal body

weight gain)

Berman et al. 1995

Dosing volume was 1 $\mathrm{mL} / \mathrm{kg}$. 
Table 3-2 Levels of Significant Exposure to Phenol - Oral

(continued)

\begin{tabular}{lll}
\hline & & $\begin{array}{r}\text { Exposu } \\
\text { Duration }\end{array}$ \\
$\begin{array}{l}\text { Key to } \\
\text { Figure }\end{array}$ & $\begin{array}{l}\text { Species } \\
\text { (Strain) }\end{array}$ & $\begin{array}{r}\text { Freque } \\
\text { (Rout })\end{array}$ \\
\hline & & \\
17 & $\begin{array}{l}\text { Rat } \\
\text { (Fischer- 344) }\end{array}$ & $14 \mathrm{~d} / \mathrm{d}$ \\
& &
\end{tabular}

$$
\text { (GW) }
$$

Neurological

18 Rat

$\begin{array}{ll}\text { Rat } & \text { once } \\ \text { (Sprague- } & (\mathrm{G})\end{array}$

Dawley)

19 Rat

once

(Fischer-344) (GW)

20

$\begin{array}{ll}\text { Rat } & 14 \mathrm{~d} \\ \text { (Fischer- 344) } & 1 \mathrm{x} / \mathrm{d}\end{array}$

(GW)

21

$\begin{array}{ll}\text { Mouse } & 10 \mathrm{~d} \\ \text { (CD-1) } & \text { Gd 6-15 } \\ & 1 \mathrm{x} / \mathrm{d} \\ & (\mathrm{GW})\end{array}$

\section{Developmental}

22 Rat
(Fischer- 344) Gd 6-19
(GW)

LOAEL

Reference

Chemical Form

Comments

System (mg/kg/day) (mg/kg/day)

(mg/kg/day)

Berman et al. 1995

$207 \mathrm{M}$ (muscle twitching, convulsions, coma)

$120 \mathrm{~F}$ (mild-to-severe whole-body tremors, decreased motor activity)

Moser et al. 1995

Dosing volume was 1 $\mathrm{mL} / \mathrm{kg}$.

Dosing volume was 1 $\mathrm{mL} / \mathrm{kg}$.

NOAEL is for weight and histopathology of the spleen.

Dosing volume not reported.

$40 \mathrm{~F}$ (increased rearing)

$70 \mathrm{~F}$ $140 \mathrm{~F}$ (mild tremors on the first
3 days of dosing)

$280 \mathrm{~F}$ (tremors, ataxia in pregnant dams)

NTP 1983b

53.3 $\mathrm{F}$ (significant decrease in the number of live-born

pups, associated with

severe respiratory effects

in the dams)
Dosing volume was 1 $\mathrm{mL} / \mathrm{kg}$.
Narotsky and Kavlock 1995 
Table 3-2 Levels of Significant Exposure to Phenol - Oral

(continued)

\begin{tabular}{|c|c|c|c|c|c|c|c|c|}
\hline \multirow[b]{2}{*}{$\begin{array}{l}\text { Key to } \\
\text { Figure }\end{array}$} & \multirow[b]{2}{*}{$\begin{array}{l}\text { Species } \\
\text { (Strain) }\end{array}$} & \multirow{2}{*}{$\begin{array}{l}\text { Exposurel } \\
\text { Durationl } \\
\text { Frequency } \\
\text { (Route) }\end{array}$} & \multirow[b]{2}{*}{ System } & \multirow[b]{2}{*}{$\begin{array}{l}\text { NOAEL } \\
\text { (mg/kg/day) }\end{array}$} & \multicolumn{2}{|c|}{ LOAEL } & \multirow[b]{2}{*}{$\begin{array}{l}\text { Reference } \\
\text { Chemical Form }\end{array}$} & \multirow[b]{2}{*}{ Comments } \\
\hline & & & & & $\begin{array}{r}\text { Less Serious } \\
(\mathrm{mg} / \mathrm{kg} / \mathrm{day})\end{array}$ & $\begin{array}{l}\text { Serious } \\
\text { (mg/kg/day) }\end{array}$ & & \\
\hline 23 & $\begin{array}{l}\text { Rat } \\
(C D)\end{array}$ & $\begin{array}{l}10 \mathrm{~d} \\
\mathrm{Gd} 6-15 \\
1 \times / d \\
(\mathrm{GW})\end{array}$ & & $60 \mathrm{~F}$ & $\begin{array}{c}120 \mathrm{~F} \text { ( } 7 \% \text { decrease in average } \\
\text { fetal body weight) }\end{array}$ & & NTP 1983a & $\begin{array}{l}\text { Dosing volume was } 5 \\
\mathrm{~mL} / \mathrm{kg} \text {. }\end{array}$ \\
\hline 24 & $\begin{array}{l}\text { Rat } \\
\text { (Sprague- } \\
\text { Dawley) }\end{array}$ & $\begin{array}{l}10 \mathrm{~d} \\
\mathrm{Gd} 6-15 \\
3 \times / d \\
(\mathrm{GW})\end{array}$ & & 120 & $\begin{array}{l}360 \text { (decreased fetal weight } \\
\text { and decrease ossification } \\
\text { sites) }\end{array}$ & & York 1997 & $\begin{array}{l}\text { Dosing volume was } 10 \\
\mathrm{~mL} / \mathrm{kg} \text {. }\end{array}$ \\
\hline 25 & $\begin{array}{l}\text { Mouse } \\
(\mathrm{CD}-1)\end{array}$ & $\begin{array}{l}10 \mathrm{~d} \\
\mathrm{Gd} 6-15 \\
1 \mathrm{x} / \mathrm{d} \\
(\mathrm{GW})\end{array}$ & & $140 \mathrm{~F}$ & & $\begin{array}{l}280 \mathrm{~F} \text { ( } 18 \% \text { decreased fetal } \\
\text { body weight, cleft palate } \\
8 / 214)\end{array}$ & NTP 1983b & $\begin{array}{l}\text { Dosing volume was } 10 \\
\mathrm{~mL} / \mathrm{kg} \text {. }\end{array}$ \\
\hline
\end{tabular}

$(G W)$ 
Table 3-2 Levels of Significant Exposure to Phenol - Oral

(continued)

\begin{tabular}{|c|c|c|c|c|c|c|c|c|}
\hline \multirow[b]{2}{*}{$\begin{array}{l}\text { Key to } \\
\text { Figure } \\
\end{array}$} & \multirow[b]{2}{*}{$\begin{array}{l}\text { Species } \\
\text { (Strain) }\end{array}$} & \multirow{2}{*}{$\begin{array}{l}\text { Exposurel } \\
\text { Durationl } \\
\text { Frequency } \\
\text { (Route) }\end{array}$} & \multirow[b]{2}{*}{ System } & \multirow[b]{2}{*}{$\begin{array}{l}\text { NOAEL } \\
\text { (mg/kg/day) }\end{array}$} & \multicolumn{2}{|c|}{ LOAEL } & \multirow[b]{2}{*}{$\begin{array}{l}\text { Reference } \\
\text { Chemical Form }\end{array}$} & \multirow[b]{2}{*}{ Comments } \\
\hline & & & & & $\begin{array}{r}\text { Less Serious } \\
(\mathrm{mg} / \mathrm{kg} / \text { day })\end{array}$ & $\begin{array}{l}\text { Serious } \\
\text { (mg/kg/day) }\end{array}$ & & \\
\hline \multicolumn{9}{|c|}{$\begin{array}{l}\text { INTERMEDIATE EXPOSURE } \\
\text { Systemic }\end{array}$} \\
\hline \multirow[t]{9}{*}{26} & $\begin{array}{l}\text { Rat } \\
\text { (Fischer- } 344\end{array}$ & $\begin{array}{l}13 \mathrm{wk} \\
\text { 4) } \mathrm{ad} \mathrm{lib} \\
(\mathrm{W})\end{array}$ & Resp & $1694 \mathrm{~F}$ & & & $\mathrm{NCl} 1980$ & $\begin{array}{l}\text { NOAELs are for organ } \\
\text { weight and } \\
\text { histopathology. }\end{array}$ \\
\hline & & & Cardio & $1694 \mathrm{~F}$ & & & & \\
\hline & & & Gastro & $1694 \mathrm{~F}$ & & & & \\
\hline & & & Musc/skel & $1694 \mathrm{~F}$ & & & & \\
\hline & & & Hepatic & $1694 \mathrm{~F}$ & & & & \\
\hline & & & Renal & $1694 \mathrm{~F}$ & & & & \\
\hline & & & Endocr & $1694 \mathrm{~F}$ & & & & \\
\hline & & & Dermal & $1694 \mathrm{~F}$ & & & & \\
\hline & & & $\mathrm{Bd} \mathrm{Wt}$ & $\begin{array}{l}467^{\mathrm{C}} \mathrm{M} \\
508 \mathrm{~F}\end{array}$ & $\begin{aligned} & 1556 \text { M }(16 \% \text { decrease in body } \\
& \text { weight gain associated } \\
& \text { with decreased water } \\
& \text { intake) }\end{aligned}$ & $\begin{aligned} & 1694 \mathrm{~F} \text { (26\% decrease in body } \\
& \text { weight gain associated } \\
& \text { with decreased water } \\
& \text { intake) }\end{aligned}$ & & \\
\hline \multirow[t]{3}{*}{27} & $\begin{array}{l}\text { Rat } \\
\text { (Sprague- } \\
\text { Dawley) }\end{array}$ & $\begin{array}{l}10 \text { wk } \\
2-\text { gen } \\
\text { ad lib } \\
(\mathrm{W})\end{array}$ & Hemato & $321 \mathrm{~F}$ & & & Ryan et al. 2001 & $\begin{array}{l}\text { NOAELs are for } \\
\text { histopathology of liver } \\
\text { and kidney. }\end{array}$ \\
\hline & & & Hepatic & $321 \mathrm{~F}$ & & & & \\
\hline & & & Renal & $321 \mathrm{~F}$ & & & & \\
\hline
\end{tabular}


Table 3-2 Levels of Significant Exposure to Phenol - Oral

(continued)

\begin{tabular}{|c|c|c|c|c|c|c|c|c|}
\hline \multirow[b]{2}{*}{$\begin{array}{l}\text { Key to } \\
\text { Figure } \\
\end{array}$} & \multirow[b]{2}{*}{$\begin{array}{l}\text { Species } \\
\text { (Strain) }\end{array}$} & \multirow{2}{*}{$\begin{array}{l}\text { Exposurel } \\
\text { Durationl } \\
\text { Frequency } \\
\text { (Route) }\end{array}$} & \multirow[b]{2}{*}{ System } & \multirow[b]{2}{*}{$\begin{array}{l}\text { NOAEL } \\
\text { (mg/kg/day) }\end{array}$} & \multicolumn{3}{|c|}{ LOAEL } & \multirow[b]{2}{*}{ Comments } \\
\hline & & & & & $\begin{array}{r}\text { Less Serious } \\
(\mathrm{mg} / \mathrm{kg} / \text { day) }\end{array}$ & $\begin{array}{l}\text { Serious } \\
\text { (mg/kg/day) }\end{array}$ & $\begin{array}{l}\text { Reference } \\
\text { Chemical Form }\end{array}$ & \\
\hline \multirow[t]{6}{*}{28} & $\begin{array}{l}\text { Mouse } \\
(\mathrm{CD}-1)\end{array}$ & $\begin{array}{l}28 d \\
\text { ad lib } \\
(W)\end{array}$ & Resp & $33.6 \mathrm{M}$ & & & Hsieh et al. 1992 & $\begin{array}{l}\text { NOAELs are for } \\
\text { histopathology. }\end{array}$ \\
\hline & & & Cardio & $33.6 \mathrm{M}$ & & & & \\
\hline & & & Hemato & \multicolumn{5}{|c|}{$\begin{array}{c}1.8 \mathrm{M}(32 \% \text { decrease in } \mathrm{RBC} \\
\text { count })\end{array}$} \\
\hline & & & Hepatic & $33.6 \mathrm{M}$ & & & & \\
\hline & & & Renal & $33.6 \mathrm{M}$ & & & & \\
\hline & & & $\mathrm{Bd} \mathrm{Wt}$ & $33.6 \mathrm{M}$ & & & & \\
\hline
\end{tabular}


Table 3-2 Levels of Significant Exposure to Phenol - Oral

(continued)

\begin{tabular}{llllr}
\hline Key to Species & $\begin{array}{c}\text { Exposurel } \\
\text { Durationl } \\
\text { Frequency } \\
\text { (Route) }\end{array}$ & System & $\begin{array}{c}\text { NOAEL } \\
\text { (mg/kg/day) }\end{array}$ \\
\hline Figure & (Strain) & Resp & \\
\hline & $\begin{array}{l}\text { Mouse } \\
\text { (B6C3F1) }\end{array}$ & $\begin{array}{l}13 \mathrm{wk} \\
\text { ad lib } \\
\text { (W) }\end{array}$ & 2642 F & \\
& & Cardio & $2642 \mathrm{~F}$ \\
& & Gastro & $2642 \mathrm{~F}$ \\
& & Musc/skel & $2642 \mathrm{~F}$ \\
& & Hepatic & $2642 \mathrm{~F}$ \\
& & Renal & $2642 \mathrm{~F}$ \\
& & Endocr & $2642 \mathrm{~F}$ \\
& & Dermal & $2642 \mathrm{~F}$ \\
& & Bd Wt & $741 \mathrm{M}$
\end{tabular}

LOAEL

Reference

Chemical Form

Comments

(mg/kg/day)

NOAELs are for organ

weight and

histopathology.

2468 M $(80 \%$ decrease in body weight gain associated with decreased water

intake)

NCI 1980

Immunol Lymphoret

\section{Rat $10 w k$ \\ (Sprague- ad lib \\ Dawley) (W)}

$31 \quad \begin{array}{ll}\text { Mouse } & 28 \mathrm{~d} \\ (\mathrm{CD}-1) & \text { ad lib }\end{array}$

(W)
$321 \mathrm{~F}$

Ryan et al. 2001
$1.8 \mathrm{M} \quad 6.2 \mathrm{M}$ (decreased antibody production response to SRBC)

NOAEL is for spleen and thymus histology and antibody

production against

immunization with

SRBC. 
Table 3-2 Levels of Significant Exposure to Phenol - Oral

(continued)

\begin{tabular}{|c|c|c|c|c|c|c|c|c|}
\hline \multirow[b]{2}{*}{$\begin{array}{l}\text { Key to } \\
\text { Figure }\end{array}$} & \multirow[b]{2}{*}{$\begin{array}{l}\text { Species } \\
\text { (Strain) }\end{array}$} & \multirow{2}{*}{$\begin{array}{l}\text { Exposurel } \\
\text { Duration/ } \\
\text { Frequency } \\
\text { (Route) }\end{array}$} & \multirow[b]{2}{*}{ System } & \multirow[b]{2}{*}{$\begin{array}{l}\text { NOAEL } \\
\text { (mg/kg/day) }\end{array}$} & \multicolumn{2}{|c|}{ LOAEL } & \multirow[b]{2}{*}{$\begin{array}{l}\text { Reference } \\
\text { Chemical Form }\end{array}$} & \multirow[b]{2}{*}{ Comments } \\
\hline & & & & & $\begin{array}{r}\text { Less Serious } \\
\text { (mg/kg/day) }\end{array}$ & $\begin{array}{l}\text { Serious } \\
\text { (mg/kg/day) }\end{array}$ & & \\
\hline
\end{tabular}

Neurological

32 Rat $\quad 13 \mathrm{wk}$

Dawley) (W)

33 Mouse $28 d$

(CD-1) ad lib

(W)

Reproductive

$34 \quad$ Rat $13 w$

(Fischer- 344) ad lib

$1556^{\mathrm{C}} \mathrm{M}$

$1694 \mathrm{~F}$

35 Rat

(Sprague- 2-gen

Dawley) ad lib

(W)

$36 \quad$ Mouse $13 \mathrm{wk}$

(B6C3F1) ad lib

(W)

\section{Developmental}

$37 \quad$ Rat

(Sprague- 2-gen

Dawley) (W)

$301 \mathrm{M}$

$321 \mathrm{~F}$

$2468 \mathrm{M}$

$2642 \mathrm{~F}$ on week 4)

1.8 $\mathrm{M}$ (decreased levels of

dopamine in the corpus

striatum)
Beyrouty 1998

Hsieh et al. 1992

histopathology of

reproductive organs.

Ryan et al. 2001

NCI 1980

NOAEL is for $P$ males sperm parameters and F1 reproductive organ histology.

NOAEL is for

reproductive organ histopathology. 
Table 3-2 Levels of Significant Exposure to Phenol - Oral

(continued)

\begin{tabular}{|c|c|c|c|c|c|c|c|c|}
\hline \multirow[b]{2}{*}{$\begin{array}{l}\text { Key to } \\
\text { Figure } \\
\end{array}$} & \multirow[b]{2}{*}{$\begin{array}{l}\text { Species } \\
\text { (Strain) }\end{array}$} & \multirow{2}{*}{$\begin{array}{l}\text { Exposurel } \\
\text { Duration/ } \\
\text { Frequency } \\
\text { (Route) }\end{array}$} & \multirow[b]{2}{*}{ System } & \multirow[b]{2}{*}{$\begin{array}{l}\text { NOAEL } \\
\text { (mg/kg/day) }\end{array}$} & \multicolumn{2}{|c|}{ LOAEL } & \multirow[b]{2}{*}{$\begin{array}{l}\text { Reference } \\
\text { Chemical Form }\end{array}$} & \multirow[b]{2}{*}{ Comments } \\
\hline & & & & & \multirow[t]{2}{*}{$\begin{array}{r}\text { Less Serious } \\
(\mathrm{mg} / \mathrm{kg} / \mathrm{day}) \\
\end{array}$} & \multirow[t]{2}{*}{$\begin{array}{l}\text { Serious } \\
\text { (mg/kg/day) }\end{array}$} & & \\
\hline \multicolumn{7}{|c|}{$\begin{array}{l}\text { CHRONIC EXPOSURE } \\
\text { Systemic }\end{array}$} & & \\
\hline \multirow[t]{10}{*}{38} & $\begin{array}{l}\text { Rat } \\
\text { (Fischer- }\end{array}$ & $\begin{array}{l}103 \mathrm{wk} \\
\text { 44) } \begin{array}{l}\text { ad lib } \\
\text { (W) }\end{array}\end{array}$ & Resp & $721 \mathrm{~F}$ & & & $\mathrm{NCl} 1980$ & $\begin{array}{l}\text { NOAELs are for organ } \\
\text { weight and } \\
\text { histopathology. }\end{array}$ \\
\hline & & & Cardio & $721 \mathrm{~F}$ & & & & \\
\hline & & & Gastro & $721 \mathrm{~F}$ & & & & \\
\hline & & & Musc/skel & $721 \mathrm{~F}$ & & & & \\
\hline & & & Hepatic & $721 \mathrm{~F}$ & & & & \\
\hline & & & Renal & $721 \mathrm{~F}$ & & & & \\
\hline & & & Endocr & $721 \mathrm{~F}$ & & & & \\
\hline & & & Dermal & $721 \mathrm{~F}$ & & & & \\
\hline & & & $\mathrm{Bd} \mathrm{Wt}$ & & $\begin{array}{r}322 \mathrm{M} \text { (about } 12 \\
\text { body wei } \\
\text { with a } 20 \\
\text { water int }\end{array}$ & & & \\
\hline & & & & & $\begin{array}{r}721 \mathrm{~F} \text { (about } 17 \\
\text { body wei } \\
\text { with a } 10 \\
\text { water int }\end{array}$ & & & \\
\hline
\end{tabular}


Table 3-2 Levels of Significant Exposure to Phenol - Oral

(continued)

\begin{tabular}{|c|c|c|c|c|}
\hline $\begin{array}{l}\text { Key to } \\
\text { Figure }\end{array}$ & $\begin{array}{r}\text { Species } \\
\text { (Strain) }\end{array}$ & $\begin{array}{c}\text { Exposurel } \\
\text { Durationl } \\
\text { Frequency } \\
\text { (Route) }\end{array}$ & System & $\begin{array}{c}\text { NOAEL } \\
(\mathrm{mg} / \mathrm{kg} / \mathrm{d}\end{array}$ \\
\hline \multirow[t]{9}{*}{39} & $\begin{array}{l}\text { Mouse } \\
\text { (B6C3F1) }\end{array}$ & $\begin{array}{l}103 \mathrm{wk} \\
\text { ad lib } \\
\text { (W) }\end{array}$ & Resp & $1204 \mathrm{~F}$ \\
\hline & & & Cardio & $1204 \mathrm{~F}$ \\
\hline & & & Gastro & $1204 \mathrm{~F}$ \\
\hline & & & Musc/ske & $1204 \mathrm{~F}$ \\
\hline & & & Hepatic & $1204 \mathrm{~F}$ \\
\hline & & & Renal & $1204 \mathrm{~F}$ \\
\hline & & & Endocr & $1204 \mathrm{~F}$ \\
\hline & & & Dermal & $1204 \mathrm{~F}$ \\
\hline & & & $\mathrm{Bd} \mathrm{Wt}$ & $1204 \mathrm{~F}$ \\
\hline
\end{tabular}

Immunol Lymphoret

40 Rat $103 w k$

(Fischer- 344) ad lib

(W)

LOAEL

Reference

Chemical Form

Comments

(mg/kg/day)

NCI 1980

NOAELs are for organ

weight and

histopathology.

NOAEL is for weight and histopathology of lymphoreticular organs and tissues.

\section{$41 \quad$ Mouse $103 \mathrm{wk}$ (B6C3F1) \\ (W)}

\section{Neurological}

$42 \quad \begin{array}{ll}\text { Rat } 103 ~ w k \\ \text { (Fischer- 344) ad lib }\end{array}$ (W)
$1204 \mathrm{~F}$

$\mathrm{NCl} 1980$

NOAEL is for weight and histopathology of lymphoreticular organs. Immuno competence was not evaluated.

NOAEL is for weight and histopathology of the brain. 
Table 3-2 Levels of Significant Exposure to Phenol - Oral

(continued)

\begin{tabular}{|c|c|c|c|c|c|c|c|c|}
\hline \multirow[b]{2}{*}{$\begin{array}{l}\text { Key to } \\
\text { Figure }\end{array}$} & \multirow{2}{*}{$\begin{array}{l}\text { Species } \\
\text { (Strain) }\end{array}$} & \multirow{2}{*}{$\begin{array}{l}\text { Exposurel } \\
\text { Durationl } \\
\text { Frequency } \\
\text { (Route) }\end{array}$} & \multirow[b]{2}{*}{ System } & \multirow[b]{2}{*}{$\begin{array}{l}\text { NOAEL } \\
\text { (mg/kg/day) }\end{array}$} & \multicolumn{2}{|c|}{ LOAEL } & \multirow[b]{2}{*}{$\begin{array}{l}\text { Reference } \\
\text { Chemical Form }\end{array}$} & \multirow[b]{2}{*}{ Comments } \\
\hline & & & & & $\begin{array}{r}\text { Less Serious } \\
\text { (mg/kg/day) }\end{array}$ & $\begin{array}{l}\text { Serious } \\
\text { (mg/kg/day) }\end{array}$ & & \\
\hline 43 & $\begin{array}{l}\text { Mouse } \\
\text { (B6C3F1) }\end{array}$ & $\begin{array}{l}103 \mathrm{wk} \\
\text { ad lib } \\
\text { (W) }\end{array}$ & & $1204 \mathrm{~F}$ & & & $\mathrm{NCl} 1980$ & $\begin{array}{l}\text { NOAEL is for weight } \\
\text { and histopathology of } \\
\text { the brain. }\end{array}$ \\
\hline \multicolumn{9}{|c|}{ Reproductive } \\
\hline 44 & $\begin{array}{l}\text { Rat } \\
\text { (Fischer- 344) }\end{array}$ & $\begin{array}{l}103 \text { wk } \\
\text { ad lib } \\
(W)\end{array}$ & & $721 \mathrm{~F}$ & & & NCl 1980 & $\begin{array}{l}\text { NOAEL is for weight } \\
\text { and histopathology of } \\
\text { reproductive organs of } \\
\text { males and females. } \\
\text { Fertility was not } \\
\text { assessed. }\end{array}$ \\
\hline 45 & $\begin{array}{l}\text { Mouse } \\
\text { (B6C3F1) }\end{array}$ & $\begin{array}{l}103 \mathrm{wk} \\
\text { ad lib } \\
(\mathrm{W})\end{array}$ & & $1204 \mathrm{~F}$ & & & $\mathrm{NCl} 1980$ & $\begin{array}{l}\text { NOAEL is for weight } \\
\text { and histopathology of } \\
\text { reproductive organs of } \\
\text { males and females. } \\
\text { Fertility was not } \\
\text { assessed. }\end{array}$ \\
\hline
\end{tabular}

assessed.

a The number corresponds to entries in Figure 3-2.

b Used to derive an acute-duration oral MRL of $1 \mathrm{mg} / \mathrm{kg} / \mathrm{day}$; the MRL was derived by dividing the BMDL of $125 \mathrm{mg} / \mathrm{kg} / \mathrm{day}$ by an uncertainty factor of 100 (10 for animal to human extrapolation and 10 for human variability).

c Differences in levels of health effects and cancer effects between male and females are not indicated in Figure 3-2. Where such differences exist, only the levels of effect for the most sensitive gender are presented.

ad lib = ad libitum; Bd Wt = body weight; Cardio = cardiovascular; $d=$ day $(\mathrm{s}) ;$ Endocr = endocrine; $\mathrm{F}=$ Female; $(\mathrm{G})=$ gavage; Gastro = gastrointestinal; gd = gestational day; gen = generation; Gn pig = guinea pig; $(\mathrm{GO})$ = gavage in oil; $(\mathrm{GW})=$ gavage in water; Hemato = hematological; hr = hour(s); Immuno = immunological; LD50 = lethal dose, $50 \%$ kill; LOAEL $=$ lowest-observed-adverse-effect level; $\mathrm{M}=$ male; $\mathrm{min}=$ minute(s); Musc/skel = musculoskeletal; NOAEL = no-observed-adverse-effect level; NS = not specified; RBC = red blood cell; Resp = respiratory; SRBC = sheep red blood cells; $x=$ time(s); (W) = drinking water; wk = week(s); yr = year(s) 
Figure 3-2 Levels of Significant Exposure to Phenol - Oral

Acute ( $\leq 14$ days)

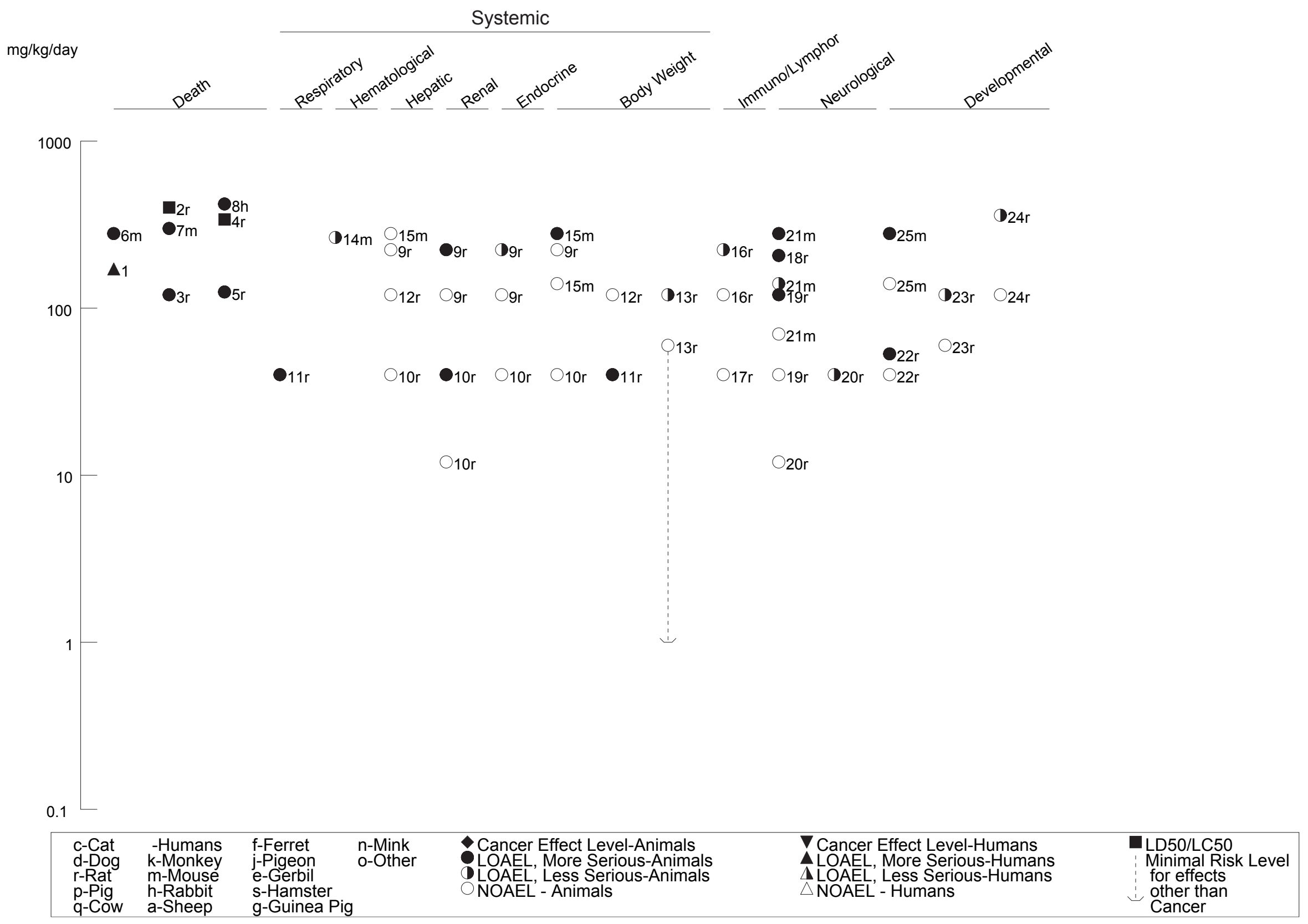

\begin{tabular}{|c|c|c|c|c|c|c|}
\hline $\begin{array}{l}\text { c-Cat } \\
\text { d-Dog } \\
\text { r-Rat } \\
\text { p-Pig } \\
\text { q-Cow }\end{array}$ & $\begin{array}{l}\text {-Humans } \\
\text { k-Monkey } \\
\text { m-Mouse } \\
\text { h-Rabbit } \\
\text { a-Sheep }\end{array}$ & $\begin{array}{l}\text { f-Ferret } \\
\text { j-Pigeon } \\
\text { e-Gerbil } \\
\text { s-Hamster } \\
\text { g-Guinea Pig }\end{array}$ & $\begin{array}{l}\text { n-Mink } \\
\text { o-Other }\end{array}$ & $\begin{array}{l}\text { Cancer Effect Level-Animals } \\
\text { LOAEL, More Serious-Animals } \\
\text { LOAEL, Less Serious-Animals } \\
\text { NOAEL - Animals }\end{array}$ & $\begin{array}{l}\nabla \text { Cancer Effect Level-Humans } \\
\Delta \text { LOAEL, More Serious-Humans } \\
\Delta \text { LOAEL, Less Serious-Humans } \\
\triangle \text { NOAEL - Humans }\end{array}$ & $\begin{array}{l}\text { LD50/LC50 } \\
\text { Minimal Risk Level } \\
\text { for effects } \\
\text { other than } \\
\text { Cancer }\end{array}$ \\
\hline
\end{tabular}


Figure 3-2 Levels of Significant Exposure to Phenol - Oral (Continued) Intermediate (15-364 days)

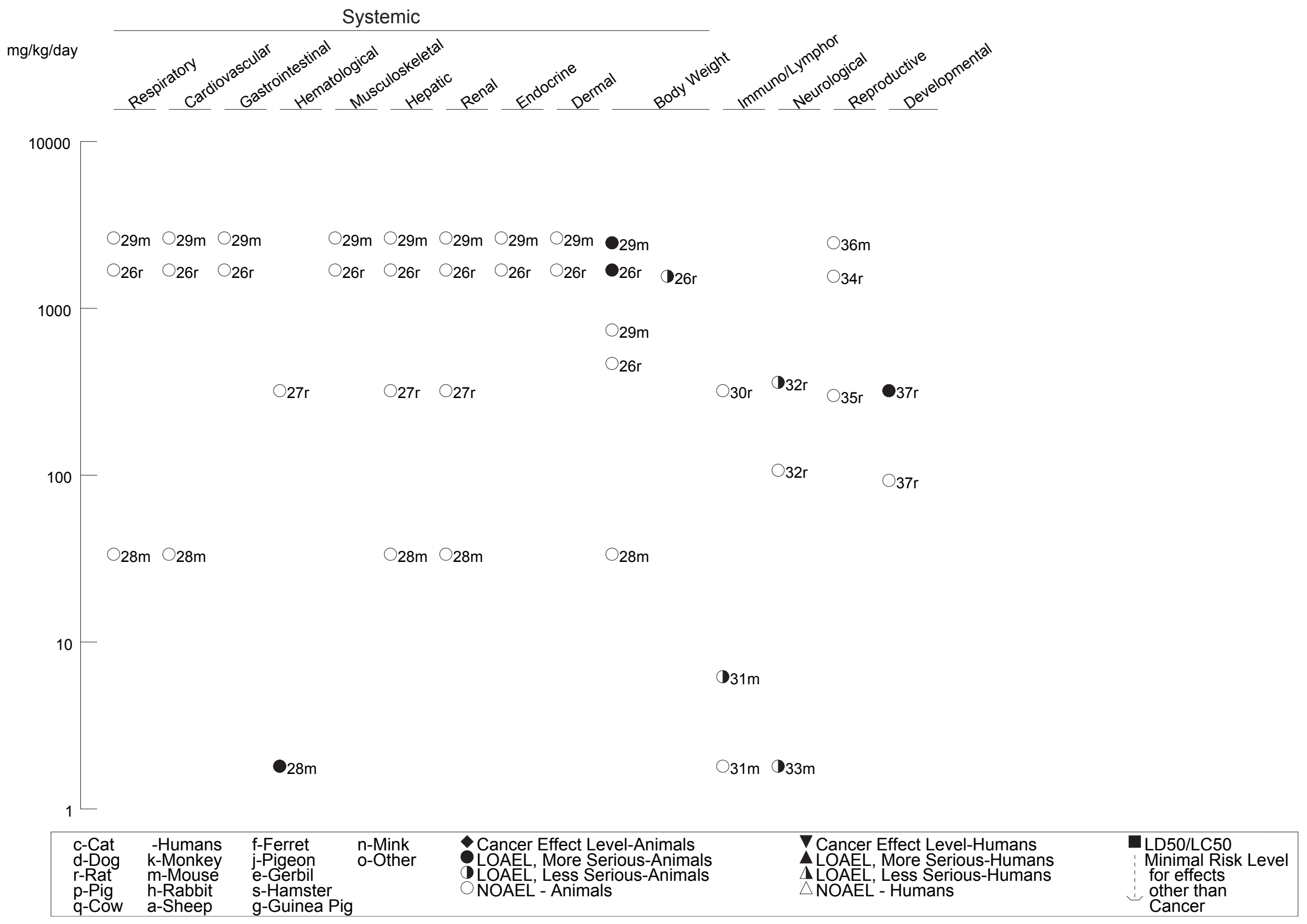


Figure 3-2 Levels of Significant Exposure to Phenol - Oral (Continued) Chronic ( $\geq 365$ days)

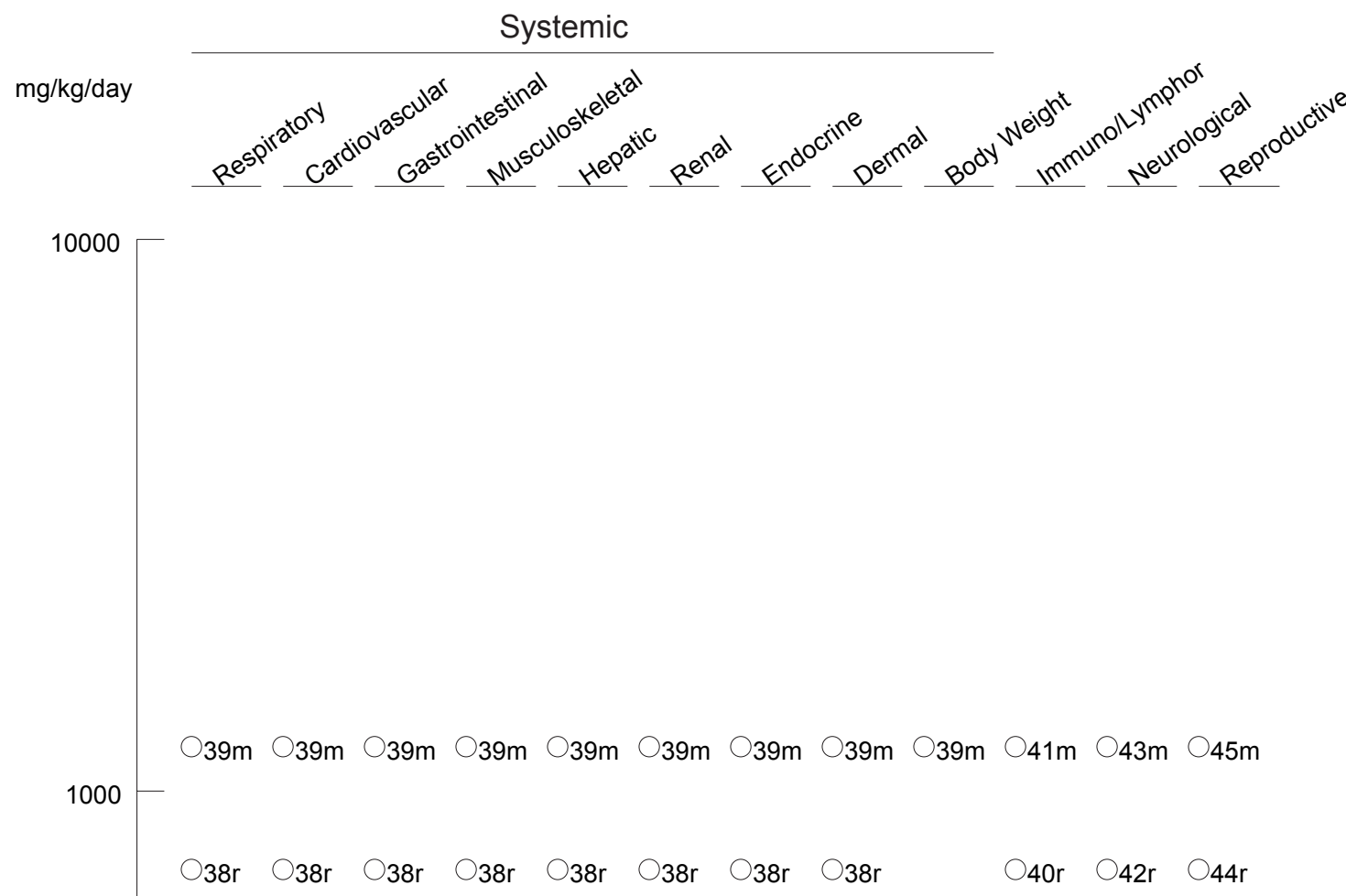

$38 \mathrm{r}$

$100 \longleftarrow$

\begin{tabular}{|c|c|c|c|c|c|c|}
\hline $\begin{array}{l}\text { c-Cat } \\
\text { d-Dog } \\
\text { r-Rat } \\
\text { p-Pig } \\
\text { q-Cow }\end{array}$ & $\begin{array}{l}\text {-Humans } \\
\text { k-Monkey } \\
\text { m-Mouse } \\
\text { h-Rabbit } \\
\text { a-Sheep }\end{array}$ & $\begin{array}{l}\text { f-Ferret } \\
\text { j-Pigeon } \\
\text { e-Gerbil } \\
\text { s-Hamster } \\
\text { g-Guinea Pig }\end{array}$ & $\begin{array}{l}\text { n-Mink } \\
\text { o-Other }\end{array}$ & $\begin{array}{l}\text { Cancer Effect Level-Animals } \\
\text { LOAEL, More Serious-Animals } \\
\text { LOAEL, Less Serious-Animals } \\
\text { NOAEL - Animals }\end{array}$ & $\begin{array}{l}\nabla \text { Cancer Effect Level-Humans } \\
\Delta \text { LOAEL, More Serious-Humans } \\
\Delta \text { LOAEL, Less Serious-Humans } \\
\triangle \text { NOAEL - Humans }\end{array}$ & $\begin{array}{l}\text { LD50/LC50 } \\
\text { Minimal Risk Level } \\
\text { for effects } \\
\text { other than } \\
\text { Cancer }\end{array}$ \\
\hline
\end{tabular}


the $795 \mathrm{mg} / \mathrm{kg}$ dose, and five of five animals died within 2 hours following 1,580 mg/kg dose. From these data, the investigators estimated an oral $\mathrm{LD}_{50}$ of $650 \mathrm{mg} / \mathrm{kg}$.

No effect on survival was observed in mice treated with phenol in the drinking water at doses up to $33.6 \mathrm{mg} / \mathrm{kg} /$ day for 28 days (Hsieh et al. 1992). Survival was not affected in rats and mice treated with phenol in drinking water for 13 or 103 weeks (NCI 1980). Both species were treated with drinking water concentrations up to $10,000 \mathrm{mg} / \mathrm{L}$ in the 13-week study (maximum doses in $\mathrm{mg} / \mathrm{kg} / \mathrm{day}: 1,694$ for female rats, 1,556 for male rats; 2,643 for female mice, 2,468 for male mice), and up to 5,000 $\mathrm{mg} / \mathrm{L}$ in the 103-week study (maximum doses in $\mathrm{mg} / \mathrm{kg} /$ day: 721 for female rats, 645 for male rats; 1,204 for female mice, 1,180 for female mice).

In most studies, a specific cause of death was not reported, but common signs preceding death included convulsions, coma, and respiratory arrest. The $\mathrm{LD}_{50}$ values and doses resulting in death from each reliable study in each species in the acute-duration category are recorded in Table 3-2 and plotted in Figure 3-2.

\subsubsection{Systemic Effects}

The highest NOAEL values and all LOAEL values from each reliable study for systemic effects in each species and duration category are recorded in Table 3-2 and plotted in Figure 3-2.

Respiratory Effects. Stajduhar-Caric (1968) reported on a case in which a woman who ingested approximately 10-20 g of phenol, became comatose and died within a matter of hours. During the course of the poisoning and treatment, initially an increase in respiration was observed, then irregularities in breathing, and finally cessation of respiration. An autopsy revealed marked hyperemia of the tracheal and bronchial mucous membranes, as well as pulmonary edema. According to Deichmann and Keplinger (1981), the progression of impacts on the respiratory system summarized above are typical of oral poisonings in humans, although often the intermediate stages are characterized by a decrease in respiration rate and magnitude. According to these authors, in acute intoxication, death usually results from respiratory failure. Pulmonary congestion and edema were reported in a man who died following phenol poisoning (Soares and Tift 1982). In another case of fatal ingestion of phenol, autopsy reported white froth in the nostrils, upper airway, trachea, and bronchi as well as edema and fluid in the lungs (Lo Dico et al. 1989). Inflammatory changes in the lungs also were observed in a more recent fatal case of ingestion of phenol (Tanaka et al. 1998). 
Dyspnea and rales were observed in pregnant rats treated by gavage with phenol in water on GDs 6-19 (Narotsky and Kavlock 1995). The respiratory effects were observed at both 40 and $53.3 \mathrm{mg} / \mathrm{kg} / \mathrm{day}$. Gross pathological examinations did not reveal any adverse changes in the lungs of mice treated with phenol in drinking water at doses of 1.8, 6.2, or $33.6 \mathrm{mg} / \mathrm{kg} /$ day for 28 days (Hsieh et al. 1992).

In a study reported by the National Cancer Institute (NCI 1980), rats exposed to $16-1,694 \mathrm{mg} / \mathrm{kg} / \mathrm{day}$ $(100-10,000 \mathrm{mg} / \mathrm{L})$ and mice exposed to $25-2,642 \mathrm{mg} / \mathrm{kg} /$ day $(100-10,000 \mathrm{mg} / \mathrm{L})$ phenol in drinking water exhibited no indication of histopathological effects on the respiratory system after 13 weeks of exposure. No histological abnormalities of the respiratory tract were observed in rats or mice exposed to 2,500 or 5,000 ppm phenol in drinking water for 103 weeks $(\mathrm{mg} / \mathrm{kg} /$ day doses: 322 or 645 for male rats; 360 or 721 for female rats; 590 or 1,180 for male mice; 602 or 1,204 for female mice) (NCI 1980).

Cardiovascular Effects. In a recent report on the clinical treatment of phenol poisoning, Langford et al. (1998) provide a summary of a case report in which a woman accidentally consumed an ounce of $89 \%$ phenol that had mistakenly been given to her in preparation for an in-office procedure. Her immediate reaction upon consuming the phenol was to clutch her throat and collapse, and within 30 minutes, she was comatose and had gone into respiratory arrest. Treatment was initiated with an endotracheal intubation. Ventilation with a bag and mask led to the detection of a lamp oil odor. Within an hour, she developed ventricular tachycardia, which responded to cardioversion; however, she subsequently developed (in the first 24 hours) supraventricular and ventricular dysrhythmias, metabolic acidosis, and experienced a grand mal seizure. After a 15-day hospital stay, she was completely recovered with no evidence of impaired motility or compromised gastrointestinal or cardiovascular systems.

Gross pathological examinations did not reveal any adverse changes in the hearts of mice treated with phenol in drinking water at doses of 1.8, 6.2, or $33.6 \mathrm{mg} / \mathrm{kg}$ /day for 28 days (Hsieh et al. 1992). In a study reported by the NCI (1980), rats exposed to 16-1,694 mg/kg/day (100-10,000 ppm) and mice exposed to $25-2,642 \mathrm{mg} / \mathrm{kg} /$ day $(100-10,000 \mathrm{ppm})$ phenol in drinking water exhibited no indication of histopathological effects on the heart after 13 weeks of exposure. Histological abnormalities of the heart were not evident in rats after 103 weeks of exposure to 322 or $645 \mathrm{mg} / \mathrm{kg} /$ day for males or 360 or $721 \mathrm{mg} / \mathrm{kg} /$ day for females $(2,500$ or $5,000 \mathrm{ppm})$ or in mice after exposure to 590 or $1,180 \mathrm{mg} / \mathrm{kg} / \mathrm{day}$ for males or 602 or $1,204 \mathrm{mg} / \mathrm{kg} /$ day for females $(2,500$ or 5,000 ppm). Cardiovascular function was not evaluated in these studies. 
Gastrointestinal Effects. In a study on the clinical treatment of phenol poisoning, Langford et al. (1998) provide a summary of a case report in which a woman accidentally consumed an ounce of $89 \%$ phenol that had mistakenly been given to her in preparation for an in-office procedure. Her immediate reaction upon consuming the phenol was to clutch her throat and collapse, and within 30 minutes, she was comatose and had gone into respiratory arrest. Treatment was initiated with an endotracheal intubation, which revealed her mouth and hypopharynx to be white. Esophagitis and upper gastrointestinal bleeding occurred in the first week, and an examination of the esophagus on day 8 revealed hyperkeratosis, erythema, and a friable mucosa. After a 15-day hospital stay, she was completely recovered with no evidence of impaired motility or compromised gastrointestinal system. A male who ingested a solution containing phenol and cresol and eventually died did not show macroscopic lesions of the esophagus and stomach, but histological examination revealed exfoliation of the mucosa of the esophagus and coagulative necrosis of the gastric mucosa (Boatto et al. 2004). Coagulation of the gastric mucosa was also reported in a fatal case of poisoning with phenol (Soares and Tift 1982). Erosive duodenal gastritis was also observed in a woman who ingested about $70 \mathrm{~mL}$ of a $42-52 \%$ phenol solution (Kamijo et al. 1999). Other fatal cases have described mucous changes in the digestive organs (Tanaka et al. 1998) and crusted corrugated appearance of the stomach (Lo Dico et al. 1989).

In a retrospective study of 158 persons exposed to phenol in drinking water for several weeks following an accidental spill of phenol, significantly $(\mathrm{p}<0.01)$ increased gastrointestinal symptoms (mouth sores, nausea, diarrhea) were reported by 17 of the 39 most highly-exposed individuals (Baker et al. 1978). Exposure concentrations for the most highly-exposed group were $>0.1 \mathrm{mg} / \mathrm{L}$, and the study authors estimated phenol intake during this period as $10-240 \mathrm{mg} /$ person/day $(0.14-3.4 \mathrm{mg} / \mathrm{kg} /$ day assuming a $70-\mathrm{kg}$ body weight). Symptom rates were not increased among 61 persons exposed to concentrations of $\leq 0.1 \mathrm{mg} / \mathrm{L}(0.003 \mathrm{mg} / \mathrm{kg} /$ day assuming $2 \mathrm{~L}$ water per day and a $70-\mathrm{kg}$ body weight). Dermal exposure was not considered in these estimates of dose. A similar study was conducted by Jarvis et al. (1985) among 344 English households whose drinking water contaminated with phenol. An unexposed group (250 households) served as control. The day of the contamination incident, the concentration of phenol in a high-exposure area (250 households) was estimated to be $10 \mu \mathrm{g} / \mathrm{L}$, whereas in a low-exposure area (94 households), the concentration was $4.7 \mu \mathrm{g} / \mathrm{L}$. The day after the incident, the phenol concentration in the water from both areas was $4.7 \mu \mathrm{g} / \mathrm{L}$, and 2 days after the incident, it was $0.9 \mu \mathrm{g} / \mathrm{L}$. Chlorophenols, which formed upon chlorination of the water, were detected at a higher concentration of phenol and followed a similar pattern as phenol. Since the concentration of phenol and chlorophenols were similar in the two exposed areas, the two exposed areas were combined in the analysis. Exposed individuals had 
significantly higher incidences of gastrointestinal illness (i.e., diarrhea, nausea, vomiting, and abdominal pain) than the unexposed group, and the onset of symptoms coincided with the period of elevated concentrations of phenol in the drinking water. Moreover, stronger associations were observed among those who reported drinking the water in the exposed areas than among those who reported not drinking the water in the same areas. The specific contribution of phenol to the adverse signs and symptoms reported is difficult to determine.

A case control study of 6,913 individuals living near a Korean river contaminated with 30 tons of $100 \%$ phenol found nausea, vomiting, diarrhea, and abdominal pain among 1,824 exposed subjects compared to 1,064 unexposed subjects (Kim et al. 1994). The level of phenol measured in the two reservoirs that served the community was $0.05 \mathrm{mg} / \mathrm{L}$ after the spill, while that in the chlorinated tap water was $0.0084 \mathrm{mg} / \mathrm{L}$.

In a study reported by the NCI (1980), rats exposed up to $1,694 \mathrm{mg} / \mathrm{kg} / \mathrm{day}$ and mice exposed up to $2,642 \mathrm{mg} / \mathrm{kg} /$ day phenol in drinking water exhibited no indication of histopathological effects on the gastrointestinal system after 13 weeks of exposure. No histological abnormalities of the gastrointestinal tract were observed in rats or mice exposed to phenol in drinking water that provided doses of approximately 720 and 1,200 mg/kg/day, respectively, for 103 weeks (NCI 1980).

Hematological Effects. No studies were located regarding hematological effects in humans following oral exposure to phenol.

A $30-60 \%$ decrease in the ratio of polychromatic to normochromatic erythrocytes was observed in the bone marrow of pregnant mice treated by gavage with a single dose of $265 \mathrm{mg} / \mathrm{kg}$ phenol in water on GD 13 (Ciranni et al. 1988). Dose-related and significant decreases in red blood cell counts were observed in mice treated with phenol in the drinking water at doses of $1.8,6.2$, or $33.6 \mathrm{mg} / \mathrm{kg} /$ day for 28 days (Hsieh et al. 1992). Red blood cell counts in cells $\times 10^{6} / \mathrm{mm}^{3}$ were 7.17 in controls, 4.9 at the low dose, 4.64 at the middle dose, and 3.23 at the high dose. A significant decrease in hematocrit was only observed at the high dose ( $48 \%$ control, $44.1 \%$ high dose), and no changes were observed in leucocyte numbers or leucocyte differentials.

No significant alterations in hematology parameters were observed in male or female rats dosed with up to 301 or $321 \mathrm{mg}$ phenol/kg/day in the drinking water, respectively, for 10 weeks (Ryan et al. 2001). 
Musculoskeletal Effects. No studies were located regarding musculoskeletal effects in humans following oral exposure to phenol.

Rats exposed up to $1,694 \mathrm{mg} / \mathrm{kg} /$ day and mice exposed up to $2,642 \mathrm{mg} / \mathrm{kg} /$ day phenol in drinking water exhibited no histological abnormalities of the bone after 13 weeks of exposure (NCI 1980). No histological abnormalities of the bone were observed in rats or mice up to $721 \mathrm{or} 1,204 \mathrm{mg} / \mathrm{kg} / \mathrm{day}$, respectively, of phenol in drinking water for 103 weeks. (NCI 1980).

Hepatic Effects. Serum markers of liver effects, bilirubin, glucose, cholesterol, and AST activity were not affected in 39 persons exposed to phenol in the drinking water at an estimated dose of 0.14$3.4 \mathrm{mg} / \mathrm{kg} /$ day for several weeks (Baker et al. 1978). Because these examinations were completed 7 months after the spill, this study does not provide conclusive evidence that there was no reversible liver damage. Autopsy of a fatal case of ingestion of phenol revealed substantial toxic changes in the liver including extension of sinusoid lumens and an increase in centrilobular increase of cytoplasmic eosinophility (Tanaka et al. 1998).

Serum markers of liver effects (lactic dehydrogenase, alkaline phosphatase, ALT, bilirubin) and histopathological changes in the liver were observed in rats given single gavage doses of $224 \mathrm{mg} / \mathrm{kg}$ or 14 daily gavage doses of $40 \mathrm{mg}$ phenol/kg in water (Berman et al. 1995). Changes in liver weight were not observed in pregnant rats treated by gavage with $120 \mathrm{mg} / \mathrm{kg} /$ day of phenol in water on GDs 6-15 (NTP 1983a), or in pregnant mice treated by gavage with $280 \mathrm{mg} / \mathrm{kg} /$ day phenol in water on GDs 6-15 (NTP 1983b).

Gross pathological examinations did not reveal any lesions in mice treated with phenol in the drinking water at a dose of $33.6 \mathrm{mg} / \mathrm{kg} /$ day for 28 days (Hsieh et al. 1992).

In a study sponsored by the National Cancer Institute (NCI 1980), rats exposed up to 1,694 mg/kg/day and mice exposed up to $2,642 \mathrm{mg} / \mathrm{kg} /$ day phenol in drinking water exhibited no histological abnormalities of the liver after 13 weeks of exposure. Similar observations were made in rats dosed with up to 301$321 \mathrm{mg}$ phenol/kg/day in the drinking water for 10 weeks (Ryan et al. 2001). No histological abnormalities of the liver were observed in rats or mice exposed up to 721 or $1,204 \mathrm{mg} / \mathrm{kg} / \mathrm{day}$ respectively, of phenol in drinking water for 103 weeks (NCI 1980). 
Renal Effects. Although not adverse, dark urine (as a result of oxidation products of phenol or a result of hemoglobin or its breakdown products in the urine) is a common symptom observed in humans exposed to phenol. In persons exposed to about $0.14-3.4 \mathrm{mg} / \mathrm{kg} / \mathrm{day}$ phenol in drinking water for several weeks after an accidental spill, dark urine was reported by $17.9 \%$ of the most highly-exposed individuals, while only $3.4 \%$ of the controls reported the effect (Baker et al. 1978). This difference was not statistically significant. A 3.3-fold increase in the prevalence of dark urine was reported by persons exposed to unspecified doses of phenol after an accidental spill in Korea (Kim et al. 1994). Autopsy of an individual who ingested phenol showed interstitial edema and renal tubular hemorrhage (Tanaka et al. 1998). Extensive renal autolysis was also observed in a fatal case of poisoning with phenol (Soares and Tift 1982).

Renal tubular necrosis, protein casts, and papillary hemorrhage were not observed in rats treated with a single gavage dose of $120 \mathrm{mg} / \mathrm{kg}$ phenol in water, but were seen in $60 \%$ of animals examined at the next highest dose of $224 \mathrm{mg} / \mathrm{kg}$ (Berman et al. 1995). No histopathological changes in the kidney were observed after 14 daily doses of $12 \mathrm{mg} / \mathrm{kg} /$ day, but were observed in 3 of 8 animals given 14 daily doses of $40 \mathrm{mg} / \mathrm{kg} /$ day (Berman et al. 1995).

Gross pathological examinations did not reveal any adverse changes in the kidneys of mice treated with phenol in drinking water at doses of 1.8, 6.2, or $33.6 \mathrm{mg} / \mathrm{kg} /$ day for 28 days (Hsieh et al. 1992).

Rats exposed up to $1,694 \mathrm{mg} / \mathrm{kg} /$ day and mice exposed up to $2,642 \mathrm{mg} / \mathrm{kg} /$ day of phenol in drinking water exhibited no indication of histopathological effects on the kidney after 13 weeks of exposure (NCI 1980). Rats exposed to up to 301-321 mg phenol/kg/day in the drinking water for 10 weeks showed no significant histopathological effects in the kidneys (Ryan et al. 2001).

No compound-related histological changes in the kidneys were observed in rats or mice exposed up to 721 or $1,204 \mathrm{mg} / \mathrm{kg} /$ day, respectively, of phenol in drinking water for 103 weeks (NCI 1980). A higher incidence of inflammation of the kidney was reported in male rats exposed to $624 \mathrm{mg} / \mathrm{kg} / \mathrm{day}(96 \%)$ than in controls (74\%); however, because of the high incidence of inflammation in the controls, it is impossible to ascertain whether this was related to the exposure to phenol (NCI 1980). A high age-related incidence of inflammation is expected in male rats of the Fischer-344 strain used in this study. Kidney function, including glomerular filtration rate and glomerular sieving, however, was not evaluated in this study. Furthermore, histological examination was limited to standard light microscopic examinations which 
would not have detected functionally significant glomerular abnormalities like disruption of the glomerular basement membrane or immune complex deposition.

Endocrine Effects. Autopsy of a subject who died following ingestion of a solution containing phenol showed interstitial hemorrhage in the pancreas and adrenal glands (Tanaka et al. 1998). No further information regarding effects of phenol on endocrine-related end points was located.

Unspecified microscopic changes were observed in the adrenal glands of rats given a single gavage dose of $224 \mathrm{mg} / \mathrm{kg}$ phenol in water, but no changes were described in rats treated similarly with doses $\leq 120 \mathrm{mg} / \mathrm{kg} /$ day (Berman et al. 1995). No adrenal gland effects were observed in rats 14 daily gavage doses of 4, 12, or $40 \mathrm{mg} / \mathrm{kg}$ (Berman et al. 1995).

Rats exposed up to $1,694 \mathrm{mg} / \mathrm{kg} / \mathrm{day}$ and mice exposed up to $2,642 \mathrm{mg} / \mathrm{kg} / \mathrm{day}$ phenol in drinking water exhibited no histopathological changes in the pancreas, pituitary, adrenal glands, thyroid, or parathyroid after 13 weeks of exposure (NCI 1980). Exposure-related histopathological changes in the pancreas, pituitary, adrenal glands, thyroid, or parathyroid were also not observed in rats and mice exposed to 2,500 or 5,000 ppm phenol in drinking water for 103 weeks (NCI 1980). Estimated $\mathrm{mg} / \mathrm{kg} /$ day doses were 322 or 645 for male rats, 360 or 721 for female rats, 590 or 1,180 for male mice, and 602 or 1,204 for female mice.

Dermal Effects. Skin rash and mouth sores were reported in persons living near a site with contaminated well water resulting from an overturned tanker car carrying 37,900 L of 100\% phenol (Baker et al. 1978). The level of phenol in the drinking water of this cohort was $>0.1 \mathrm{mg} / \mathrm{L}$, and while substantial oral exposure probably occurred, dermal exposure cannot be ruled out. Increases in the prevalence of skin rashes and sore throats were reported by persons drinking water from a river contaminated by an accidental spill of phenol (Kim et al. 1994). Because the water was chlorinated before use, the effect may also have been a result of exposure to chlorophenol. Perioral chemical burns from phenol were observed in a woman who ingested approximately $70 \mathrm{~mL}$ of a $42-52 \%$ phenol solution (Kamijo et al. 1999). Spiller et al. (1993) also reported oral and esophageal burns in 17 out of 52 patients following ingestion of a disinfectant containing $26 \%$ phenol.

Rats exposed up to $1,694 \mathrm{mg} / \mathrm{kg}$ /day and mice exposed up to $2,642 \mathrm{mg} / \mathrm{kg} /$ day phenol in drinking water exhibited no histopathological changes in the skin after 13 weeks of exposure (NCI 1980). Exposure- 
related histopathological changes in the skin were also not observed in rats and mice exposed up to 721 or $1,204 \mathrm{mg} / \mathrm{kg} /$ day, respectively, phenol in drinking water for 103 weeks (NCI 1980).

Ocular Effects. No studies were located regarding ocular effects in humans or animals following oral exposure to phenol.

Body Weight Effects. No effects on body weight were observed in rats treated with a single gavage dose of $224 \mathrm{mg} / \mathrm{kg}$ phenol in water or 14 daily gavage doses of $40 \mathrm{mg} / \mathrm{kg}$ (Berman et al. 1995; Moser et al. 1995). Maternal body weight gain was approximately $20 \%$ lower in rats treated by gavage with 40 or $53.3 \mathrm{mg} / \mathrm{kg} /$ day phenol in water on GDs 6-19 (Narotsky and Kavlock 1995). Maternal body weight gain was $67 \%$ lower than controls in mice treated by gavage with $280 \mathrm{mg} / \mathrm{kg} /$ day phenol in water on GDs 615, with no effects on body weight gain observed at $140 \mathrm{mg} / \mathrm{kg} /$ day (NTP 1983b). Body weight gain was not affected in pregnant rats treated by gavage with $120 \mathrm{mg} / \mathrm{kg} /$ day phenol in water on GDs 6-15 (NTP 1983a). Body weight gain was significantly reduced in pregnant rats dosed with $120 \mathrm{mg}$ phenol/kg/day on GDs 6-15 (11\% for GDs 6-16 and 19\% for GDs 12-16) (York 1997). Doses of $360 \mathrm{mg} / \mathrm{kg} / \mathrm{day}$ caused a reduction in body weight gain of 38\% for GDs 6-16; the NOAEL for maternal weight gain in this study was $60 \mathrm{mg} / \mathrm{kg} /$ day. The reduction in maternal body weight gain in rats during pregnancy reported in the York (1997) study was used as the basis for derivation of an acute-duration oral MRL for phenol.

Body weight was not affected in mice treated with phenol in drinking water at a dose of $33.6 \mathrm{mg} / \mathrm{kg} / \mathrm{day}$ for 28 days (Hsieh et al. 1992). During 13-week studies in rats and mice treated with phenol in drinking water, decreased body weight gain was associated with dose-related decreases in water intake, presumably due to taste aversion (NCI 1980). In rats provided with the highest concentration (10,000 ppm), body weight gain was decreased by $26 \%$ in females at $1,694 \mathrm{mg} / \mathrm{kg} /$ day, and by $16 \%$ in males at $1,556 \mathrm{mg} / \mathrm{kg} /$ day. An effect on body weight gain was not observed in rats at 3,000 ppm (467 mg/kg/day for males, $508 \mathrm{mg} / \mathrm{kg} /$ day for females). In mice provided with the highest concentration (10,000 ppm), body weight gain was decreased by $33 \%$ in females at $2,642 \mathrm{mg} / \mathrm{kg} /$ day, and by $80 \%$ in males at $2,468 \mathrm{mg} / \mathrm{kg} /$ day. An effect on body weight gain was not observed in mice at 3,000 ppm $(741 \mathrm{mg} / \mathrm{kg} /$ day for males, $793 \mathrm{mg} / \mathrm{kg} /$ day for females). A significant decrease in body weight gain associated with a significant decrease in water consumption was also observed in male and female rats dosed with 301-321 mg/kg/day phenol in the drinking water for 10 weeks (Ryan et al. 2001). 
Decreased mean body weight associated with decreased water intake was also observed in rats in a 103-week study (NCI 1980). At the high concentration (5,000 ppm), body weight was $19 \%$ lower than controls in males $(645 \mathrm{mg} / \mathrm{kg} / \mathrm{day})$ and $17 \%$ lower than controls in females $(721 \mathrm{mg} / \mathrm{kg} / \mathrm{day})$. At the low concentration (2,500 ppm), body weight was $12 \%$ lower than controls in males (322 $\mathrm{mg} / \mathrm{kg} / \mathrm{day})$ and within $10 \%$ of controls in females $(360 \mathrm{mg} / \mathrm{kg} /$ day). Body weight was not affected in mice treated with phenol in drinking water for 103 weeks at doses up to 1,180 for males and up to $1,204 \mathrm{mg} / \mathrm{kg} / \mathrm{day}$ for females (NCI 1980).

\subsubsection{Immunological and Lymphoreticular Effects}

No studies were located regarding immunological or lymphoreticular effects in humans following oral exposure to phenol.

Necrosis or atrophy of the spleen or thymus was observed in four of six rats given a single gavage dose of $224 \mathrm{mg} / \mathrm{kg}$ of phenol in water, and one of seven rats given $120 \mathrm{mg} / \mathrm{kg} /$ day (Berman et al. 1995). Based on this effect, which was not further described, the study authors considered $224 \mathrm{mg} / \mathrm{kg}$ to be a LOAEL for immunological effects. One of eight animals given $12 \mathrm{mg} / \mathrm{kg} / \mathrm{day}$, and two of eight given $40 \mathrm{mg} / \mathrm{kg} / \mathrm{day}$ for 14 days showed these same effects.

Hsieh et al. (1992) conducted a 28-day study of the immunotoxicologic impact of phenol in which CD-1 mice were given drinking water that provided doses of phenol of approximately $0,1.8,6.2$, or $33.6 \mathrm{mg} / \mathrm{kg} /$ day. When challenged with sheep red blood cells (SRBC), a significant decrease was observed in the splenic concentration of anti-erythrocyte antibody-forming cells and in the antierythrocyte antibody titer at the two highest doses, while a significant decrease in the absolute number of anti-erythrocyte antibody-forming cells present in the spleen was observed only at the top dose. In contrast to the results of Hsieh et al. (1992), Ryan et al. (2001) reported no immunologic alterations in male rats in a drinking water study. Rats were dosed for 10 weeks with up to $301 \mathrm{mg}$ phenol/kg/day and then immunized intravenously with sheep red blood cells. Eighteen hours later, splenocytes were prepared in cell culture medium for enumeration of plaque-forming cells. Treatment with phenol had no significant effect on antibody-forming cells, and there were no significant effects on spleen or thymus weight, spleen cellularity, or spleen and thymus histology.

Rats exposed up to $1,694 \mathrm{mg} / \mathrm{kg}$ /day and mice exposed up to $2,642 \mathrm{mg} / \mathrm{kg} /$ day phenol in drinking water exhibited no histopathological changes in the bone marrow, spleen, or lymph nodes after 13 weeks of 
exposure (NCI 1980). Exposure-related histopathological changes in the bone marrow, spleen, or lymph nodes were also not observed in rats or mice exposed to estimated doses of up to 721 or $1,204 \mathrm{mg} / \mathrm{kg} / \mathrm{day}$, respectively, for 103 weeks (NCI 1980).

The highest NOAEL values and all LOAEL values from each reliable study for immunological and lymphoreticular effects in each species and duration category are recorded in Table 3-2 and plotted in Figure 3-2.

\subsubsection{Neurological Effects}

Headaches were reported 6 times more frequently by persons using drinking water contaminated with phenol than by controls (Kim et al. 1994). The water was used after chlorination; therefore, chlorophenol may have contributed to the observed effects. Fine, rapid, rhythmic, perioral movements, as well as signs of Parkinson's syndrome were observed in a woman who ingested approximately $70 \mathrm{~mL}$ of a $42-52 \%$ solution of phenol (Kamijo et al. 1999). Central nervous system depression was described in 11 out of 52 patients who ingested amounts ranging from 2 to $90 \mathrm{~mL}$ of a disinfectant solution containing $26 \%$ phenol (Spiller et al. 1993).

Acute oral phenol poisoning in rabbits and rats was characterized by muscular tremors in the head region, which eventually spread to other regions of the body, with the lower extremities being the last affected. Loss of coordination and convulsions preceded death at doses of 300-940 mg/kg (Deichmann and Witherup 1944). Liao and Oehme (1981) described tremors of the muscles around the eyes, followed by convulsions and coma, in rats after a sublethal oral dose of $207 \mathrm{mg} / \mathrm{kg}$ phenol. Mild-to-severe wholebody tremors and decreased motor activity were reported in rats given a single gavage dose of $120 \mathrm{mg} / \mathrm{kg}$ phenol in water (Moser et al. 1995). A dose of $40 \mathrm{mg} / \mathrm{kg}$ resulted in no neurological effects following a single dose, while increased rearing was reported following 14 daily doses (Moser et al. 1995). Pregnant mice treated by gavage with phenol in water on GDs 6-15 exhibited tremors and ataxia at $280 \mathrm{mg} / \mathrm{kg} / \mathrm{day}$, mild tremors on the first 3 days of dosing at $140 \mathrm{mg} / \mathrm{kg} /$ day, and no adverse neurological effects at $70 \mathrm{mg} / \mathrm{kg} /$ day (NTP 1983b).

In contrast with results from oral gavage studies, phenol administered in the drinking water was much less toxic. For example, male rats exposed to up to $309 \mathrm{mg}$ phenol $/ \mathrm{kg} /$ day in the drinking water for 13 weeks showed no significant alterations in tests of motor activity or a functional observation battery conducted throughout the exposure period (Beyrouty 1998). However, females dosed with $360 \mathrm{mg}$ phenol/kg/day 
showed a significant reduction in motor activity on week 4 of the study; no significant alterations were seen in females at $107 \mathrm{mg} / \mathrm{kg} / \mathrm{day}$. Gross and microscopic evaluation of the brain, spinal cord, and peripheral nerves was unremarkable (Beyrouty 1998).

Mice exposed for 28 days to phenol in drinking water exhibited a significant reduction in dopamine level in the corpus striatum at the $1.8 \mathrm{mg} / \mathrm{kg} /$ day dose, and significantly decreased levels of norepinephrine, serotonin, and 5-hydroxyindoleacetic acid in the hypothalamus at the $6.2 \mathrm{mg} / \mathrm{kg} / \mathrm{day}$ dose (Hsieh et al. 1992). Levels of neurotransmitters in other brain regions were also significantly altered at higher doses of phenol.

Rats exposed to $16-1,694 \mathrm{mg} / \mathrm{kg} /$ day and mice exposed to $25-2,642 \mathrm{mg} / \mathrm{kg} /$ day phenol in drinking water exhibited no abnormal histology of the brain after 13 weeks of exposure (NCI 1980). Histopathological changes in the brain were not evident after 103 weeks of exposure to 322 or $645 \mathrm{mg} / \mathrm{kg} /$ day in male rats, 360 or $721 \mathrm{mg} / \mathrm{kg} /$ day in female rats, 590 or $1,180 \mathrm{mg} / \mathrm{kg} /$ day in male mice, and 602 or 1,204 mg/kg/day in female mice (NCI 1980). However, this study did not include tests for neurological impairment or histopathological examinations of tissues in the nervous system other than the brain.

The highest NOAEL values and all LOAEL values from each reliable study for neurological effects in each species and duration category are recorded in Table 3-2 and plotted in Figure 3-2.

\subsubsection{Reproductive Effects}

No studies were located regarding reproductive effects in humans following oral exposure to phenol.

No evidence of impaired reproduction was found in rats exposed to phenol in drinking water at $<5,000 \mathrm{ppm}$ (estimated $571 \mathrm{mg} / \mathrm{kg} / \mathrm{day}$ ) for three generations or at $<1,000 \mathrm{ppm}$ (estimated $114 \mathrm{mg} / \mathrm{kg} / \mathrm{day}$ ) for five generations (Heller and Pursell 1938). Data regarding breeding habits, controls, and the methods used to evaluate the rats for reproductive impairment were not reported in sufficient detail to establish reliable NOAELs or LOAELs for presentation in Table 3-2 and Figure 3-2.

Rats exposed up to $1,694 \mathrm{mg} / \mathrm{kg} /$ day and mice exposed up to $2,642 \mathrm{mg} / \mathrm{kg} /$ day of phenol in drinking water exhibited no histopathological changes in the prostate, testes, uterus, or ovaries after 13 weeks of exposure (NCI 1980). Exposure-related histopathological changes in the prostate, testes, uterus, or 
ovaries were also not observed in rats or mice exposed up to 721 or $1,204 \mathrm{mg} / \mathrm{kg} /$ day, respectively, phenol in drinking water for 103 weeks (NCI 1980).

In a two-generation study in which rats were administered phenol in the drinking water (up to 301$321 \mathrm{mg} / \mathrm{kg} /$ day), there was a significant decrease in absolute seminal vesicle weight in parental males at $301 \mathrm{mg} / \mathrm{kg} /$ day and in absolute ovaries weight in parental females at $321 \mathrm{mg} / \mathrm{kg} / \mathrm{day}$, but were no significant alterations in gross or microscopic appearance of the reproductive organs of males and females from the parental and $F_{1}$ generations (Ryan et al. 2001). In addition, there were no significant effects on estrus frequency, testicular sperm count, or sperm motility or morphology. Significant reductions in prostate and uterine weights in all $F_{1}$ treated groups were not considered adverse effects of phenol by Ryan et al. (2001) on the basis of the absence of histological alterations and functional reproductive effects, and based on the fact that only a few animals had organ weights outside the range of concurrent control values.

Information on the effects of exposure to phenol on the genetic material of germinal cells is presented in Section 3.3, Genotoxicity.

The highest NOAEL values from each reliable study for reproductive effects in each species and duration category are recorded in Table 3-2 and plotted in Figure 3-2.

\subsubsection{Developmental Effects}

No studies were located regarding developmental effects in humans following oral exposure to phenol.

In a multi-generational study of the effect of various levels of phenol administered orally in water, Heller and Pursell (1938) saw no effect on growth, reproduction, and normal rearing of young over 5 generations of rats given concentrations of $\approx 1,000 \mathrm{mg} / \mathrm{L}$ phenol in drinking water (estimated dose of $114 \mathrm{mg} / \mathrm{kg} / \mathrm{day}$ ) nor over three generations of rats given concentrations of $\approx 5,000 \mathrm{ppm}$ (estimated dose $571 \mathrm{mg} / \mathrm{kg} / \mathrm{day}$ ).

Phenol in water was administered to pregnant rats by gavage $(5 \mathrm{~mL} / \mathrm{kg})$ at dose levels of $0,30,60$, or $120 \mathrm{mg} / \mathrm{kg} /$ day on GDs 6-15 (NTP 1983a). A dose-related decrease in fetal body weight with increasing dose was observed, with $60 \mathrm{mg} / \mathrm{kg} /$ day established as the NOAEL and $120 \mathrm{mg} / \mathrm{kg} / \mathrm{day}$ as the LOAEL. The mean reduction in fetal body weight relative to controls was approximately $7 \%$. Concurrent controls provide the most appropriate comparison for experimental data; however, it is worth noting that the 
weights of control fetuses in this study were $22 \%$ higher than those of historical controls. As litter size is known to influence fetal weight, it is possible that a larger litter size in the high-dose group may have contributed to the smaller fetal weights in that group. The data were not specifically analyzed for that potential effect. Teratogenic effects were not observed and no signs of maternal toxicity were observed at any dose level. In a preliminary range-finding study conducted by NTP (1983a), a decrease in maternal weight gain and an increased incidence of maternal mortality were observed at $>160 \mathrm{mg} / \mathrm{kg}$. Tremors, a typical symptom of phenol toxicity, were also observed.

In a study of the developmental toxicity of phenol and structurally-related chemicals, Kavlock (1990) examined the effects of $0,100,333,667$ and $1,000 \mathrm{mg} / \mathrm{kg}$ phenol given by gavage on day 11 of gestation. Phenol was administered in a 4:4:1:1 mixture of water, Tween 20, propylene glycol, and ethanol. Five variables were examined: maternal weight change (at 24 and 72 hours postdosing), litter size (postnatal day [PND] 1 and 6), perinatal loss, pup weight (on PND 1 and 6), and litter biomass (in g on PND 1 and 6).

Within these five parameters, a significant decrease in maternal weight gain was seen at the two highest doses. At these same doses, malformations involving the limbs and tail were seen. At a dose of $667 \mathrm{mg} / \mathrm{kg}$, pups in $21 \%$ of the litters were affected. At a dose of $1,000 \mathrm{mg} / \mathrm{kg}$, pups in $27 \%$ of the litters were affected. The effect on tails was one of shortening or crimping (i.e., 'kinky' tails). The hindlimb effect consisted of paralysis and/or palsy. In animals with palsy, the limb function would alternate between normal strides and several, second-long period of tetany. Because limb function matures postnatally, this effect was not evident in the newborn but required 7-10 days to become obvious.

In a subsequent study, Narotsky and Kavlock (1995) found a significant decrease in the number of liveborn pups associated with severe respiratory effects in pregnant rats treated by gavage with $53.3 \mathrm{mg} / \mathrm{kg} /$ day phenol in water on GDs 6-19. In addition, in one high-dose litter, two of four surviving pups had kinked tails; this finding was not analyzed for significance but was consistent with earlier observations (Kavlock 1990). Developmental effects were not significant at $40 \mathrm{mg} / \mathrm{kg} /$ day.

Phenol in water was administered to pregnant mice by gavage $(10 \mathrm{~mL} / \mathrm{kg})$ at dose levels of $0,70,140$, or $280 \mathrm{mg} / \mathrm{kg} /$ day on GDs 6-15 (NTP 1983b). Decreased maternal weight gain, tremors, and increased maternal mortality were observed at $280 \mathrm{mg} / \mathrm{kg} / \mathrm{day}$. In the fetuses, growth retardation, decreased prenatal viability, abnormal structural development, and an increased incidence of cleft palate were observed at $280 \mathrm{mg} / \mathrm{kg} /$ day. Developmental effects were not observed at $140 \mathrm{mg} / \mathrm{kg} /$ day. In pregnant 
mice that received $265 \mathrm{mg} / \mathrm{kg}$ phenol by gavage on GD 13, Ciranni et al. (1988) found no evidence of fetal cellular toxicity, as measured by a ratio polychromatic erythrocyte/normochromatic erythrocyte.

York (1997) conducted a study in which phenol was administered in three daily gavage doses in water to Sprague-Dawley pregnant rats in dosing volumes of $10 \mathrm{~mL} / \mathrm{kg}$ on GDs 6-15. The total daily doses were $0,60,120$, or $360 \mathrm{mg}$ phenol $/ \mathrm{kg} /$ day. Maternal end points evaluated included clinical signs, body weight, and food consumption. Dams were also observed for abortions and premature deliveries. Dams were sacrificed on GD 20 and a gross necropsy was conducted. The uterus was examined for pregnancy, number and distribution of implantations, live and dead fetuses, and early and late resorptions. Fetuses were weighed and examined for sex and gross external alterations. Half of the fetuses were examined for soft tissue alterations and the remaining fetuses were examined for skeletal alterations. In the mid-dose group, maternal body weight gain was reduced $11 \%$ for GDs $6-16$ and 19\% for GDs $12-16$, whereas in the high-dose group, body weight gain was reduced 38\% for GDs 6-16. Maternal final body weight in the high-dose group was reduced, but $<10 \%$ relative to controls. Dose-related decreases in food consumption were also observed during the dosing period. Mean fetal weight in the high-dose group was reduced $5-7 \%$ relative to controls. In addition, there was a significant decrease in ossification sites on the hindlimb metatarsals in the high-dose group, which the investigators considered of minimal biological significance. At the mid- and high-dose levels, there were increases in litters with fetuses with "any alteration" and with "any variation", but neither reached statistical significance and there were no clear dose-response relationships. No significant effects were seen regarding corpora lutea, implantations, litter sizes, live fetuses, early and late resorptions, and percent resorbed conceptuses. Based on the reduced fetal weight and delayed ossification in the high-dose group, the dose of $360 \mathrm{mg} / \mathrm{kg} /$ day is a developmental LOAEL; the developmental NOAEL is $120 \mathrm{mg} / \mathrm{kg} /$ day. Based on the reduction in body weight gain, the maternal LOAEL is $120 \mathrm{mg} / \mathrm{kg} /$ day and the NOAEL is $60 \mathrm{mg} / \mathrm{kg} /$ day.

In a two-generation reproduction study, administration of phenol in the drinking water to the parental generation (301-321 mg/kg/day) resulted in a significant reduction in $F_{1}$ pup weight (30\% by PND 21 relative to controls) and $\mathrm{F}_{2}$ pup weight (37\% by PND 21 relative to controls) (Ryan et al. 2001). There was also a decrease in percent live pups on day 4 in both generations and for days 7-21 in the $\mathrm{F}_{2}$ generation at the high-dose level. In addition, preputial separation and vaginal patency were significantly delayed at the high dose in $\mathrm{F}_{1}$ males and females, respectively. In this study, water consumption was significantly reduced at the high dose, including in females during gestation and lactation, and this was accompanied by reduced food consumption and body weight gain. A LOAEL of $321 \mathrm{mg} / \mathrm{kg} / \mathrm{day}$ can be 
defined in this study for developmental toxicity; the NOAEL for developmental toxicity was $93 \mathrm{mg} / \mathrm{kg} /$ day.

The highest NOAEL values and all LOAEL values from each reliable study for developmental effects in each species in the acute-duration category are recorded in Table 3-2 and plotted in Figure 3-2.

\subsubsection{Cancer}

No studies were located regarding carcinogenicity in humans following oral exposure to phenol.

The carcinogenicity of orally administered phenol was examined in rats and mice in a study reported by the NCI (1980). Rats and mice received 0, 2,500, or 5,000 ppm in drinking water for 103 weeks. Calculated intakes for rats were 322 and $645 \mathrm{mg} / \mathrm{kg} /$ day for males and 360 and $721 \mathrm{mg} / \mathrm{kg} /$ day for females. Calculated intakes for mice were 590 and $1,180 \mathrm{mg} / \mathrm{kg} /$ day for males and 602 and $1,204 \mathrm{mg} / \mathrm{kg} /$ day for females. Statistically significant increased incidences of pheochromocytomas of the adrenal gland and leukemia or lymphomas were observed in male rats exposed to $322 \mathrm{mg} / \mathrm{kg} /$ day $(2,500 \mathrm{ppm})$, but not in male rats exposed to $645 \mathrm{mg} / \mathrm{kg} /$ day $(5,000 \mathrm{ppm})$. No significant effects were seen in female rats or mice of either sex exposed to either exposure level. Since cancer occurred only in males of one of the two species tested and a positive dose-response relationship could not be established, these results are inconclusive regarding the carcinogenic potential of orally administered phenol.

\subsubsection{Dermal Exposure}

\subsubsection{Death}

Application of phenol to the skin can be lethal. Death occurred within 10 minutes after $\approx 25 \%$ of an individual's body surface was exposed to liquid phenol (Griffiths 1973). The cause of death was reported to be respiratory depression and cardiac arrest. In another report, an individual died after being painted with a brush that had been soaked in a solution of phenol and thoroughly washed before use (Lewin and Cleary 1982). In neither case was the dose known with sufficient accuracy to establish a lethal dose.

A 10-year-old boy who was hospitalized with serious burns was treated dermally with 7.5 L of an antiseptic solution containing $2 \%$ phenol for 2.5 days. During this period his urine became dark and his respiration became labored, fell into a coma, and died. Postmortem analysis of urine showed the presence of $200 \mathrm{mg} / \mathrm{L}$ of conjugated phenol (Cronin and Brauer 1949). Soares and Tift (1982) described two fatal 
cases attributed to absorption of phenol through intact skin. One was a 17-year-old male who died within 30 minutes of splattering a solution containing 30\% phenol over portions of his face, neck, and right trunk. The other case was a 4-week-old female who was mistakenly treated with undiluted Castellani's paint (a mixture of phenol, basic fuchsin, resorcinol, acetone, ethanol, and water) to treat seborrheic dermatitis and died 5 hours later.

Lethality associated with dermal exposure to phenol is greatly influenced by the surface area exposed as well as the concentration of the applied solution. Mortality can vary depending on concentration; a dose of $100 \%$ phenol may be less toxic than the same dose of phenol given as a diluted solution. When an undiluted dose of $0.5 \mathrm{~mL} / \mathrm{kg}$ was applied to the shaved backs of groups of five rats; one rat died, a $1 / 3$ dilution killed three rats, a 1/2 dilution killed four rats, and a 2/3 dilution killed all five rats (Conning and Hayes 1970). Conning and Hayes (1970) speculated that an undiluted solution may produce a coagulative necrosis, which would slow further penetration of phenol resulting in a smaller number of deaths than with more diluted solutions. In rats treated with $3,000 \mathrm{mg} / \mathrm{kg}$ phenol in a $6 \%$ solution over $1 / 6$ of the total body surface, all 22 treated animals died (Deichmann and Witherup 1944). Increased lethality with decreased concentration has also been observed in rabbits treated dermally with $2,000 \mathrm{mg} / \mathrm{kg} ; 95 \%$ phenol resulted in the death of $53 \%$ of treated rabbits, while $10 \%$ phenol in water resulted in the death of $100 \%$ of treated rabbits (Deichmann and Witherup 1944). The cause of death was not stated in these studies.

In rats given a single treatment of $5 \%$ phenol in water to achieve a dose of 3,000 mg/kg, 10 -day-old rats were more sensitive than 5-week-old rats or adult rats (Deichmann and Witherup 1944). Within $2-$ 14 hours after dosing, 13 of 20 10-day-old rats died; 5 of 20 -week-old rats died 2-3 hours after dosing, and 9 of 20 adult rats died $30-180$ minutes after dosing.

The dermal $\mathrm{LD}_{50}$ of undiluted phenol in rats was reported to be $669 \mathrm{mg} / \mathrm{kg}$ (Conning and Hayes 1970). The $\mathrm{LD}_{50}$ of an unspecified concentration of phenol in rabbits was reported to be $1,400 \mathrm{mg} / \mathrm{kg}$ (Vernot et al. 1977). Flickinger (1976) determined a dermal $\mathrm{LD}_{50}$ by exposing male albino rabbits to $0,252,500$, 1,000 , or $2,000 \mathrm{mg} / \mathrm{kg}$ phenol which was placed "in contact" with "abraded and intact skin for a maximum period of 24 hours." No animals died in the 0,252 , or $500 \mathrm{mg} / \mathrm{kg}$ groups whereas three of four in the $1,000 \mathrm{mg} / \mathrm{kg}$ group and all in the $2,000 \mathrm{mg} / \mathrm{kg}$ group died the first day following dosing. From these data, the authors estimated a "single dose skin penetration $\mathrm{LD}_{50}$ " of $850 \mathrm{mg} / \mathrm{kg}$. 
Among pigs treated with a single dose of $500 \mathrm{mg} / \mathrm{kg}$ of undiluted phenol on $35-40 \%$ of the total body surface (about $1,136 \mathrm{~cm}^{2} ; 0.44 \mathrm{mg} / \mathrm{cm}^{2} / \mathrm{kg}$ ), two of three died (Pullin et al. 1978). The study authors reported that a general state of lethargy, cyanosis, convulsions, and coma were observed 5-7 minutes before death.

No effects on survival were observed in mice treated dermally with an unspecified volume of 5\% phenol ( 3 times/week) or 10\% phenol (2 times/week) in acetone for 12 months (Wynder and Hoffmann 1961). Pretreatment with a single dose of 7,12-dimethylbenz[a]anthracene (7,12-DMBA) followed by phenol resulted in increased skin tumors and decreased survival.

All LOAEL and $\mathrm{LD}_{50}$ values from each reliable study are recorded in Table 3-3.

\subsubsection{Systemic Effects}

The highest NOAEL values and all LOAEL values from each reliable study for systemic effects in each species and duration category are recorded in Table 3-3.

Respiratory Effects. Pulmonary edema was observed in two fatal cases of dermal poisoning with phenol (Soares and Tift 1982). A 79-year-old man who mistakenly instilled into his nose $0.5-10 \mathrm{~mL}$ of a $89 \%$ phenol solution (approximately $0.4-9 \mathrm{~g}$ ) showed erythema and sloughing of the nasal mucosa 3 days after the accident (Durback-Morris and Scharman 1999). No further relevant information was located regarding respiratory effects in humans following dermal exposure to phenol.

Dyspnea was reported in pigs treated with a single dose of $500 \mathrm{mg} / \mathrm{kg}$ of undiluted phenol over $35-40 \%$ of the total body surface area $\left(0.44 \mathrm{mg} / \mathrm{cm}^{2} / \mathrm{kg}\right.$ ) (Pullin et al. 1978). This treatment resulted in the death of two of the three treated pigs.

Cardiovascular Effects. There have been several reports of cardiac arrhythmias associated with application of phenol solutions to the skin in connection with the surgical procedure of skin peeling (Gross 1984; Truppman and Ellenby 1979; Warner and Harper 1985). In this procedure, a mixture of phenol $(\approx 50 \% \mathrm{w} / \mathrm{v})$, hexachlorophene, and croton oil is applied to the skin while the patient is under anesthesia. In a series of 54 patients in which the whole face was peeled in 1 day, cardiac arrhythmias were reported in $39 \%$, while in a series of patients in which half the face was treated on 1 day, and the second half was treated 24 hours later, cardiac arrhythmias were reported in 22\% (Gross 1984). The 
Table 3-3 Levels of Significant Exposure to Phenol - Dermal

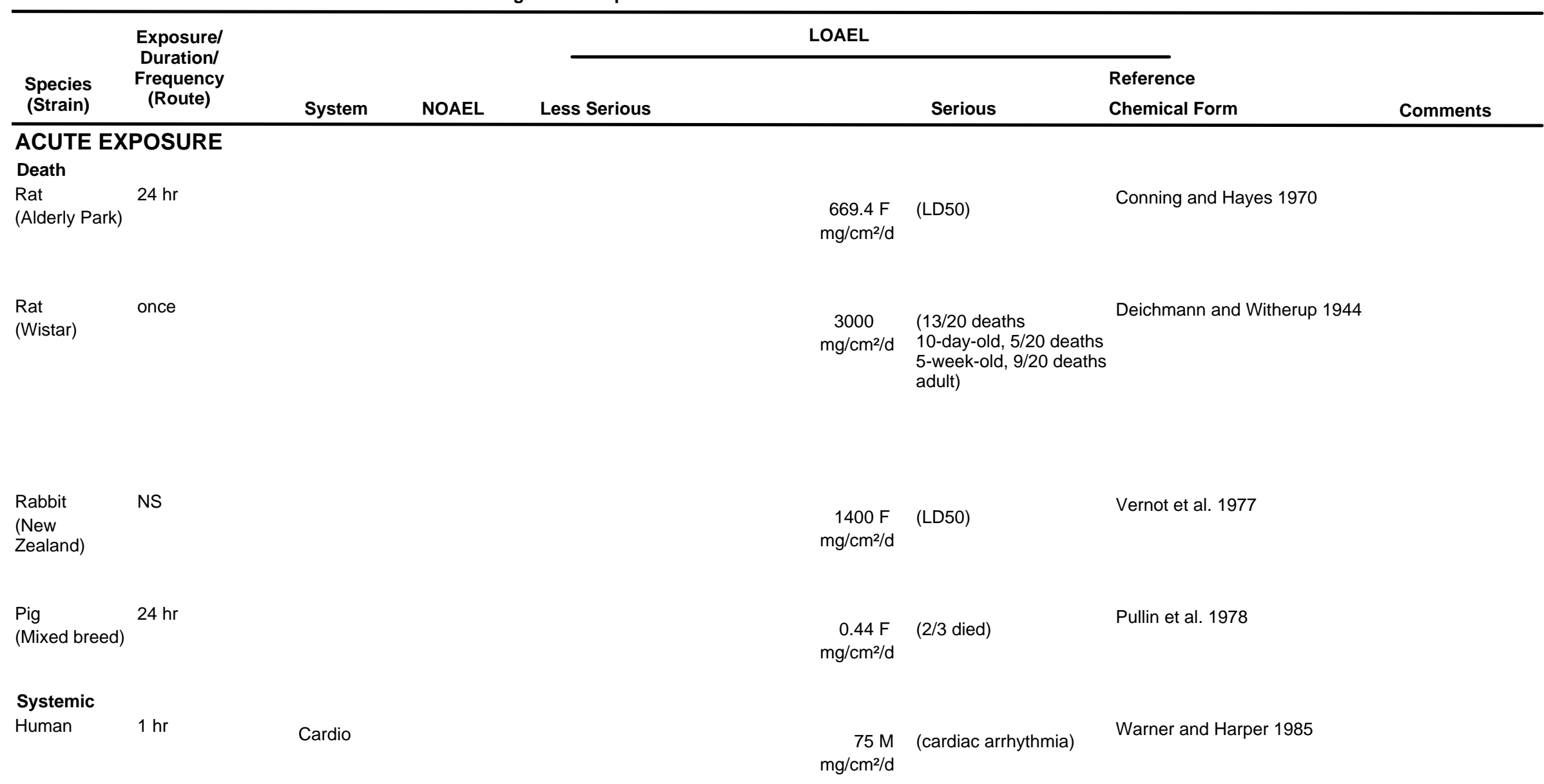


Table 3-3 Levels of Significant Exposure to Phenol - Dermal

(continued)

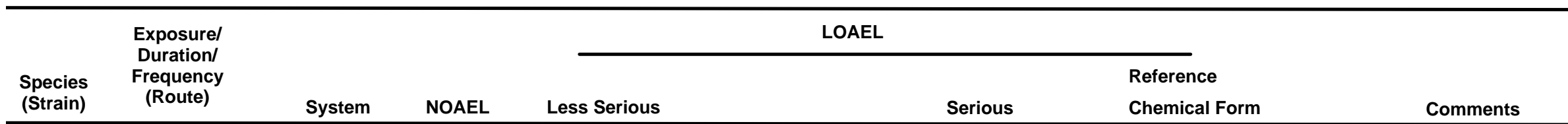

$\begin{array}{lll}\begin{array}{l}\text { Rat } \\ \text { (Alderly Park) }\end{array} & 24 \mathrm{hr} & \text { Renal }\end{array}$

(Alderly Park)

enal

Mouse once

(B6C3F1) (NS)

Mouse once

(ICR)

Rabbit

(NS)
Cardio
Dermal

Dermal

$12 \mathrm{~F}$ $\mathrm{mg} / \mathrm{cm}^{2} / \mathrm{d}$ $\mathrm{mg} / \mathrm{cm}^{2}$
$329 \mathrm{~F}$

$$
\mathrm{mg} / \mathrm{kg}
$$

(skin inflammation)

$107.1 \mathrm{~F} \quad$ (severe edema erythema) $\mathrm{mg} / \mathrm{cm}^{2} / \mathrm{d}$ coagulative necrosis,

$107.1 \mathrm{~F}$ (severe hemoglobinuria, Conning and Hayes 1970

$\mathrm{mg} / \mathrm{cm}^{2} / \mathrm{d}$ hematin casts in the

tubules) 
Table 3-3 Levels of Significant Exposure to Phenol - Dermal

(continued)

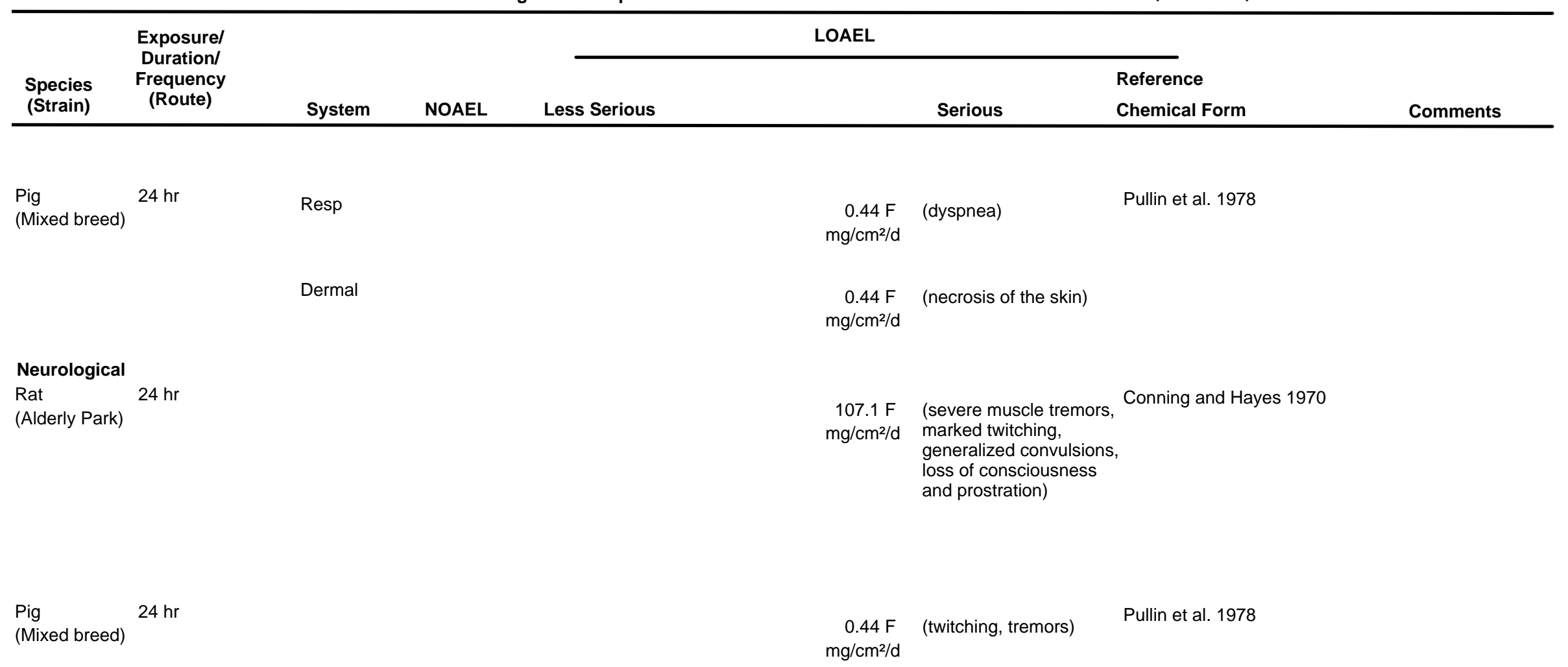

$\mathrm{mg} / \mathrm{cm}^{2} / \mathrm{d}$

Cardio = cardiovascular; $d$ = day(s); F = Female; $h r=$ hour(s); LD50 = lethal dose, 50\% kill; LOAEL = lowest-observed-adverse-effect level; $\mathrm{M}=$ male; NOAEL =

no-observed-adverse-effect level; NS = not specified; Resp = respiratory 
study author also indicated that the arrhythmias were less severe in the patients treated over a longer period of time.

Cardiac arrhythmia and bradycardia were reported in a man that splashed an unspecified concentration of a phenol-water solution over his face, chest wall, hand, and both arms (Horch et al. 1994). The cardiac effects were noted during the first 6 hours after exposure. The serum levels of phenol in $\mu \mathrm{g} / \mathrm{L}$ were 11,400 after 1 hour, 17,400 after 4 hours, and 6,000 after 8 hours. Premature heartbeats and arterial fibrillation were reported in a male who experienced a 4.5 -hour occlusive exposure to $90 \%$ phenol (Bentur et al. 1998); the concentration of phenol in serum reached a peak of $21.6 \mathrm{mg} / \mathrm{L}$.

Cardiac arrhythmia has also been noted in rabbits treated with $2 \mathrm{~mL}$ of a $50 \%$ phenol solution on a $15-\mathrm{cm}^{2}$ area $\left(23.8 \mathrm{mg} / \mathrm{cm}^{2} / \mathrm{kg}\right.$ ) (Wexler et al. 1984). Reducing plasma concentrations of phenol by forced diuresis or a longer application time reduced the cardiac effects.

Gastrointestinal Effects. During the first few days after a man splashed a phenol-water solution (concentration not stated) on his face, chest wall, hand, and both arms, he complained of nausea and vomited twice (Horch et al. 1994). A worker who was partially immersed for only a few seconds in a shallow vat containing a mixture of $40 \%$ phenol in dichloromethane, collapsed after showering and was taken to a hospital where he was found to have burns over $50 \%$ of his body. Initial observations were stable; however, after drinking fluids, he developed nausea and vomiting (Foxall et al. 1989).

No studies were located regarding gastrointestinal effects in animals following dermal exposure to phenol.

Hematological Effects. No studies were located regarding hematological effects in humans following dermal exposure to phenol.

Hemoglobinuria and hematin casts were reported in the renal tubules of rats treated dermally with $108 \mathrm{mg} / \mathrm{kg}$ phenol (Conning and Hayes 1970). These observations are indicative of red blood cell hemolysis; however, this was not confirmed with hematological examinations.

Musculoskeletal Effects. Muscle pain in the arms and legs was reported in a case of chronic phenol poisoning (Merliss 1972). The man worked in a laboratory for 13.5 years where he distilled phenol 
several times a day. During the process, heavy odors were detectable, phenol was often spilled on his clothes, and he noted skin irritation. The man recovered after 2-3 months away from the exposure.

No studies were located regarding musculoskeletal effects in animals following dermal exposure to phenol.

Hepatic Effects. Two days after a man was splashed with a phenol-water solution over his face, chest wall, hand, and both arms, serum bilirubin increased 2-fold (Horch et al. 1994). After 5 days, serum bilirubin returned to normal. An enlarged and tender liver and increased liver transaminase activity in the serum were reported in a case of chronic phenol poisoning (Merliss 1972). Lactate dehydrogenase was about 2-fold greater than normal, AST was about 21-fold greater than normal, and ALT was about 100 -fold greater than normal. The man worked in a laboratory for 13.5 years where he distilled phenol several times a day. During the process, heavy odors were detectable, phenol was often spilled on his clothes, and he noted skin irritation.

No studies were located regarding hepatic effects in animals following dermal exposure to phenol.

Renal Effects. Renal tubule cell vacuolization was described in a fatal case of dermal poisoning with phenol (Soares and Tift 1982). A case of acute renal failure was reported by Foxall et al. (1989) in a worker who accidentally fell into a shallow vat containing a mixture of phenol (40\%) in dichloromethane. The worker was partially immersed for only a few seconds and avoided ingesting any of the solution. He showered immediately, subsequently collapsed and was admitted to the hospital with surface burns over $50 \%$ of his body (involving the face, chest, genitals, and both legs). Following admission he became anuric and plasma creatinine levels rose. He was transferred to the regional renal unit where he was diagnosed with phenol- induced burns, acute tubular necrosis, and fluid overload. For the first 2 weeks, the patient demonstrated amino aciduria, glycosuria, and lactic aciduria consistent with renal cortical necrosis. This was followed by a period of polyuria revealing a biochemical pattern consistent with renal papillary damage. Treatment consisted of administration of a diuretic intravenously and hemodialysis daily for a week followed by an additional 18 days of hemodialysis at gradually increasing intervals. The patient was discharged 42 days after admission once renal clinical chemistry values had return to normal, although nuclear magnetic resonance (NMR) spectroscopic analysis still revealed abnormalities consistent with renal papillary damage. One year after the incident, the patient was still polyuric. 
Dark urine was reported in a case of chronic phenol poisoning (Merliss 1972). The man worked in a laboratory for 13.5 years where he distilled phenol several times a day. During the process, heavy odors were detectable, phenol was often spilled on his clothes, and he noted skin irritation. The study authors indicated that the urine was so dark that it suggested hemoglobinuria. Glucose was present in the urine, although the urine was negative for homogentistic acid (a substance whose presence can cause urine to darken upon standing) and urobilinogen. The urine cleared 2-3 months after the subject was removed from phenol exposure. Dark urine was also observed in a man who spilled $90 \%$ phenol over an occluded area of the skin and kept the area unattended for 4.5 hours (Bentur et al. 1998).

Hemoglobinuria and hematin casts in the distal convoluted tubules and tubular lumens located in the medulla and papilla were reported in rats after a single dermal exposure to $107 \mathrm{mg} / \mathrm{kg}$ liquid phenol (Conning and Hayes 1970). These phenomena are probably related to red blood cell lysis and increased glomerular filtration of hemoglobin. Hemoglobinuria is characteristic of lethal or near-lethal exposures by the dermal route.

Dermal Effects. Application of phenol to the skin of humans results in dermal inflammation and necrosis (Horch et al. 1994; Merliss 1972; Truppman and Ellenby 1979). Data concerning minimal effective exposure levels in humans were not found. NIOSH (1983) conducted a survey in an Oregon hospital in response to concerns about respiratory problems and contact dermatitis in housekeeping staff members who were exposed frequently to germicidal solutions containing phenol and other solvents (formaldehyde, cellosolve, ethanolamine). The housekeeping staff reported significantly more symptoms of cough, itching, sinus problems, and dermatitis than did other employees. Air concentrations of phenol in the work areas were below the limit of detection $(<0.01 \mathrm{ppm})$. Urinary phenol levels in the housekeeping staff members were not significantly different from those of the other employees. Thus, while it is likely that the employees came into contact with irritants, the cause of the reported symptoms could not be attributed to phenol or any other specific substance in the work environment. Therefore, this study is not recorded in Table 3-3.

Application of $0.1 \mathrm{~mL}$ of molten phenol $/ \mathrm{kg}(\approx 100 \mathrm{mg} / \mathrm{kg}$ ) (Brown et al. 1975) or $107 \mathrm{mg} / \mathrm{kg}$ (Conning and Hayes 1970) to the skin of rats for 24 hours (surface area not reported) produced severe edema, erythema, and necrosis. In pigs, application of $500 \mathrm{mg} / \mathrm{kg}$ molten phenol to $35-40 \%$ of the body surface $\left(0.44 \mathrm{mg} / \mathrm{cm}^{2} / \mathrm{kg}\right)$ resulted in skin discoloration after 20-30 minutes of exposure and severe necrosis after 8 hours of exposure. Two of three pigs died within 95 minutes after exposure (Pullin et al. 1978). Necrosis, hyperemia of superficial dermal vessels, and dense perivascular infiltration of lymphocytes and 
neutrophils were noted in the skin of pigs treated dermally with an unspecified amount of $89 \%$ phenol (Hunter et al. 1992). The dose-effect relationship and time course for skin irritation and inflammation have been studied in mice (Patrick et al. 1985). The end point examined was swelling (increased thickness) of the ear after dermal application to the ear pinna. Application of $12 \mathrm{mg} / \mathrm{cm}^{2} / \mathrm{kg}$ of phenol to the ear resulted in swelling in four of nine mice within 1 hour after application. Severity of skin irritation increased as the concentration of the applied phenol solution increased. Swelling persisted for 6 weeks after application of $18 \mathrm{mg} / \mathrm{cm}^{2} / \mathrm{kg}$. Swelling was observed in only one of eight mice treated with $12 \mathrm{mg} / \mathrm{cm}^{2} / \mathrm{kg}$ phenol. Application of $3.5 \mathrm{mM}$ phenol $/ \mathrm{kg}$ to a $3-\mathrm{cm}^{2}$ shaved dorsal area from mice resulted in inflammation, as evidenced by an increase in skin bi-fold thickness and recruitment of inflammatory cells (Murray et al. 2007). This was accompanied by oxidation of glutathione and protein thiols and decreased vitamin $\mathrm{E}$ and total antioxidant reserves in the skin. Application of an unspecified amount of a 1:6 or 1:9 phenol:water solution to the skin of guinea pigs for 1 minute resulted in erythema and increased skin vascular permeability indicated by dye permeability (Steele and Wilhelm 1966).

Skin crusts were reported on mice exposed repeatedly to $5 \mathrm{mg}$ phenol as a $5 \%(\mathrm{w} / \mathrm{v})$ solution for 32 weeks, whereas skin ulceration was observed in mice exposed to $5 \mathrm{mg}$ phenol as a $20 \%(\mathrm{w} / \mathrm{v})$ solution (Salaman and Glendenning 1957). The skin ulceration healed in 4 weeks after the end of the exposure. In a 52 -week study, mice were exposed 2 times each week to 42 or $83 \mathrm{mg} / \mathrm{kg}$ of phenol in a 5 or $10 \%$ solution in benzene (Boutwell and Bosch 1959). Severe skin damage was reported after 36 weeks in the mice exposed to $83 \mathrm{mg} / \mathrm{kg}$. Skin papillomas were reported in mice exposed at $42 \mathrm{mg} / \mathrm{kg}$. Because phenol was applied in benzene which is also a skin irritant, this study is not presented in Table 3-3.

Direct application of phenol to the inner ear of rats has resulted in external otitis, inner ear damage (Schmidt et al. 1990), and inflammation of the tympanic membrane (Schmidt and Hellström 1993). These studies were conducted because phenol has been used as a topical anesthetic in infected ears.

Ocular Effects. No studies were located regarding ocular effects in humans following dermal exposure to phenol.

A modified Draize test was used to assess ocular damage resulting from application of 5\% phenol to the center of the cornea in New Zealand rabbits (Murphy et al. 1982). The eyes of one group of rabbits were irrigated with water 30 seconds after exposure, while the eyes of another group were unirrigated. Conjunctivitis developed in all treated groups and lasted through the 7 days of observation. Corneal opacities became apparent in four of nine rabbits 24 hours after phenol application in unirrigated eyes, but 
only 1 hour after application in four of nine rabbits receiving irrigation. The opacities lasted through the 7-day observation period in the unirrigated eyes, but were cleared by day 7 in the irrigated eyes. Based on these observations, phenol was designated as a severe eye irritant in unirrigated eyes, and as a moderate eye irritant in irrigated eyes (Murphy et al. 1982).

Body Weight Effects. A man chronically exposed to phenol at a laboratory where he distilled it several times a day was 71.5 inches tall, weighed 135 pounds, and was described as emaciated (Merliss 1972). Loss of appetite and a slow weight loss were symptoms that the subject reported during the 13.5 years he worked at the laboratory. During the distillation process, heavy odors were detectable, phenol was often spilled on his clothes, and he noted skin irritation.

No studies were located regarding body weight effects in animals following dermal exposure to phenol.

\subsubsection{Immunological and Lymphoreticular Effects}

No studies were located regarding immunological or lymphoreticular effects in humans or animals following dermal exposure to phenol.

\subsubsection{Neurological Effects}

Fatal dermal exposure to an $80 \%$ phenol solution in a 24 -year-old man being treated for skin rash was characterized by severe convulsions prior to death (Lewin and Cleary 1982). A child who suffered accidental dermal poisoning with phenol became listless and developed seizures before death (Soares and Tift 1982). Seizures were also reported in a young adult who splattered a solution containing $30 \%$ of phenol over portions of his face, neck, and right trunk and later died (Soares and Tift 1982). A man who spilled 90\% phenol over his foot and shoe had hypalgesia and hypoesthesia of the affected area in addition to confusion, vertigo, and faintness (Bentur et al. 1998).

Muscle tremors and convulsions are characteristic effects of acute dermal phenol toxicity in laboratory animals. Tremors that developed into convulsions and prostration were reported in rats exposed to 107 mg/kg liquid phenol; application surface areas were not reported (Conning and Hayes 1970). In pigs, application of $500 \mathrm{mg} / \mathrm{kg}$ over $35-40 \%$ of the body surface $\left(0.44 \mathrm{mg} / \mathrm{cm}^{2} / \mathrm{kg}\right)$ resulted in muscular tremors in the head region within 3-5 minutes of exposure (Pullin et al. 1978). This was followed by dilation of the pupils, loss of coordination, and excess salivation and nasal discharge within 5 minutes of exposure. It was followed by convulsions, coma, and death 5-7 minutes after exposure in two of three 
pigs. Direct application of a dose of $38 \mathrm{mg} / \mathrm{kg}$ phenol to the inner ear resulted in a reduced threshold for auditory brainstem response (Schmidt et al. 1990).

No studies were located regarding the following health effects in humans or animals after dermal exposure to phenol:

\subsubsection{Reproductive Effects}

\subsubsection{Developmental Effects}

\subsubsection{Cancer}

No studies were located regarding cancer in humans following dermal exposure to phenol.

In a study of the promoting effects of phenol, mice were exposed to 9,10-dimethyl-1,2-benzanthracene (9,10-DMBA) (300 $\mu \mathrm{g})$ followed by weekly dermal exposure to $5 \mathrm{mg}$ phenol in either a 5 or $20 \%$ phenol solution in acetone for 32 weeks (Salaman and Glendenning 1957). Exposure to 9,10-DMBA followed by phenol ( 5 or 20\%) resulted in a significantly greater incidence of tumors, including carcinomas, than exposure to $20 \%$ phenol alone; tumors, but no carcinomas, resulted from exposure to $20 \%$ phenol, and no tumors resulted from exposure to 5\% phenol. Application of 5\% phenol alone resulted in skin "crusting" at the site of application, whereas $20 \%$ phenol resulted in skin ulceration. The study authors concluded that phenol was an effective tumor promoter after a single application of 9,10-DMBA. Although this study did not include a group of animals that had been exposed to 9,10-DMBA alone, the authors indicated that previous work done in their laboratory provided the data from such animals and that it was thus the comparison between such historical information and the information from this study which led to their conclusion about the promotional effects of phenol.

A similar promoting activity was observed when an unspecified volume of $10 \%$ phenol in acetone was placed on the backs of mice 2 times/week for 12 months and when 5\% phenol in acetone was placed on the backs of mice 3 times/week for 12 months (Wynder and Hoffmann 1961).

Additional studies indicate that phenol applied to the skin is a cancer promoter and possibly a complete carcinogen (i.e., promoter and initiator) in mice. Boutwell and Bosch (1959) examined the carcinogenic effects of phenol in several strains of mice. Mice were exposed to a single dermal application of 9,10-DMBA ( $75 \mathrm{mg}$ ) followed by repeated dermal applications of a 5 or $10 \%$ phenol solution in benzene 
(42 or $83 \mathrm{mg} / \mathrm{kg} /$ treatment), twice each week for 52 weeks. Two other experimental groups of mice were exposed to 9,10-DMBA alone or phenol alone. Severe skin damage, decreased body weight, and increased mortality were observed in phenol-treated animals. Sutter strain mice (inbred for three generations for susceptibility to the initiator 9,10-DMBA) treated with 9,10-DMBA followed by $10 \%$ phenol developed papillomas (95\% in 13 weeks) and carcinomas (43\% in 42 weeks) at a much higher incidence than mice treated with 9,10-DMBA alone (14\% with papillomas at 42 weeks; no carcinomas), or phenol alone (36\% with papillomas at 52 weeks; no carcinomas). One fibrosarcoma was observed after 52 weeks of exposure to phenol alone. An elevated incidence of papilloma was also observed in CAF1, C3H, and Holtzmann mice exposed to 9,10-DMBA followed by phenol, and in Holtzmann mice exposed to $10 \%$ phenol alone. The promoting effect of phenol was dose related; application of $5 \%$ phenol $(41.7 \mathrm{mg} / \mathrm{kg}$ ) following 9,10-DMBA treatment resulted in fewer tumors than a similar protocol using 9,10-DMBA followed by $10 \%$ phenol $(83.3 \mathrm{mg} / \mathrm{kg})$. Phenol elicits skin tumors in mice even without treatment with 9,10-DMBA. Ten out of 30 albino mice treated twice weekly for 12 weeks with a $20 \%$ phenol solution in dioxane developed papilloma of the skin; also, 8 out of 30 mice treated with $10 \%$ phenol solution in benzene for 15 weeks developed papilloma, and 3 developed carcinoma of the skin (Boutwell and Bosch 1959). Because the phenol was administered in benzene or dioxane, both of which are skin irritants and/or de-fatting agents, this study is not presented in Table 3-3.

The effect of phenol on benzo[a]pyrene (B[a]P) carcinogenicity has been examined (Van Duuren and Goldschmidt 1976; Van Duuren et al. 1971, 1973). Dermal application of $3 \mathrm{mg}$ phenol in acetone simultaneously with $5 \mu \mathrm{g}$ B[a]P resulted in significantly fewer tumors than application of $\mathrm{B}[\mathrm{a}] \mathrm{P}$ alone. Application surface areas were not reported and could not be estimated from the description of the application procedure. Mice treated dermally with B[a]P followed by dermal application of brewed tea on alternate days over a period of 55 days developed epithelial cell carcinoma or exhibited various stages of squamous cell tumors (Kaiser 1967). The brewed tea contained an unspecified level of phenol, the presumed cancer promoter in this experiment, as well as cresols and dimethylphenols.

\subsection{GENOTOXICITY}

Phenol has been evaluated for genotoxicity in both in vivo (Table 3-4) and in vitro (Table 3-5) test systems. End points evaluated in in vivo mammalian test systems include chromosomal aberrations, micronucleus, and deoxyribonucleic acid (DNA) synthesis. Several different cell types have been monitored, including bone marrow, liver, and renal cell. Both positive and negative results have been reported for in vivo genotoxicity tests. In vitro studies have been conducted in prokaryotic and eukaryotic 
Table 3-4. Genotoxicity of Phenol In Vivo

\begin{tabular}{|c|c|c|c|}
\hline Species (test system) & End point & Results & Reference \\
\hline \multicolumn{4}{|l|}{ Mammalian cells: } \\
\hline Mouse bone marrow & Chromosomal aberration & - & $\begin{array}{l}\text { Barale et al. 1990; Chen and } \\
\text { Eastmond 1995a; Pashin et al. } \\
1987\end{array}$ \\
\hline Mouse bone marrow & Chromosomal aberration & + & Shelby and Witt 1995 \\
\hline Mouse spermatocytes & Chromosomal aberration & + & Bulsiewicz 1977 \\
\hline $\begin{array}{l}\text { Bone marrow from pregnant } \\
\text { mice }\end{array}$ & Micronucleus & + & Ciranni et al. 1988 \\
\hline Mouse fetal liver cells & Micronucleus & - & Ciranni et al. 1988 \\
\hline Mouse bone marrow & Micronucleus & + & $\begin{array}{l}\text { Li et al. 2005; Shelby and Witt } \\
1995\end{array}$ \\
\hline Mouse bone marrow & Micronucleus & - & $\begin{array}{l}\text { Barale et al. 1990; Gocke et al. } \\
1981\end{array}$ \\
\hline $\begin{array}{l}\text { Mouse tubular renal and liver } \\
\text { epithelial }\end{array}$ & DNA synthesis & + & Amlacher and Rudolph 1981 \\
\hline Rat testes & DNA synthesis & - & Skare and Schrotel 1984 \\
\hline Rat liver & DNA synthesis & - & Miyagawa et al. 1995 \\
\hline \multicolumn{4}{|l|}{ Insects: } \\
\hline Drosophila & Micronucleus & - & $\begin{array}{l}\text { Gocke et al. 1981; Sturtevant } \\
1952\end{array}$ \\
\hline
\end{tabular}

$+=$ positive response $;-=$ negative response; DNA = deoxyribonucleic acid 


\section{Table 3-5. Genotoxicity of Phenol In Vitro}

\begin{tabular}{|c|c|c|c|c|}
\hline \multirow[b]{2}{*}{ Species (test system) } & \multirow[b]{2}{*}{ End point } & \multicolumn{2}{|c|}{ Results } & \multirow[b]{2}{*}{ Reference } \\
\hline & & $\begin{array}{l}\text { With } \\
\text { activation }\end{array}$ & $\begin{array}{l}\text { Without } \\
\text { activation }\end{array}$ & \\
\hline \multicolumn{5}{|l|}{ Prokaryotic organisms: } \\
\hline Salmonella typhimurium & Gene mutation & - & - & $\begin{array}{l}\text { Florin et al. 1980; } \\
\text { Haworth et al. 1983; } \\
\text { Kubo et al. 2002; Pool } \\
\text { and Lin } 1982\end{array}$ \\
\hline S. typhimurium & Gene mutation & + & - & Gocke et al. 1981 \\
\hline Escherichia coli & Gene mutation & - & - & Nagel et al. 1982 \\
\hline E. coli & Gene mutation & No data & + & Demerec et al. 1951 \\
\hline \multicolumn{5}{|l|}{ Eukaryotic organisms: } \\
\hline Aspergillus & $\begin{array}{l}\text { Chromosomal } \\
\text { aberration }\end{array}$ & No data & + & Crebelli et al. 1987 \\
\hline $\begin{array}{l}\text { V79 Chinese hamster } \\
\text { cells }\end{array}$ & Gene mutation & + & - & $\begin{array}{l}\text { Paschin and Bahitova } \\
1982\end{array}$ \\
\hline $\begin{array}{l}\text { Chinese hamster ovary } \\
\text { cells }\end{array}$ & Micronuclei & + & + & Miller et al. 1995 \\
\hline $\begin{array}{l}\text { Chinese hamster ovary } \\
\text { cells (DNA strand breaks) }\end{array}$ & $\begin{array}{l}\text { Chromosomal } \\
\text { aberration }\end{array}$ & No data & - & Sze et al. 1996 \\
\hline $\begin{array}{l}\text { Crucian (goldfish) } \\
\text { erythrocytes }\end{array}$ & DNA damage & No data & + & Li et al. 2005 \\
\hline $\begin{array}{l}\text { Mouse lymphoma (DNA } \\
\text { strand breaks) }\end{array}$ & $\begin{array}{l}\text { Chromosomal } \\
\text { aberration }\end{array}$ & No data & - & $\begin{array}{l}\text { Pellack-Walker and } \\
\text { Blumer } 1986\end{array}$ \\
\hline Mouse spermatocytes & DNA damage & No data & + & Li et al. 2005 \\
\hline Rat liver mitochondria & DNA synthesis & No data & - & Schwartz et al. 1985 \\
\hline $\begin{array}{l}\text { Syrian hamster embryo } \\
\text { cells }\end{array}$ & Gene mutation & No data & + & Tsutsui et al. 1997 \\
\hline $\begin{array}{l}\text { Syrian hamster embryo } \\
\text { cells }\end{array}$ & $\begin{array}{l}\text { Chromosomal } \\
\text { aberration }\end{array}$ & No data & + & Tsutsui et al. 1997 \\
\hline $\begin{array}{l}\text { Syrian hamster embryo } \\
\text { cells }\end{array}$ & $\begin{array}{l}\text { Sister chromatid } \\
\text { exchanges }\end{array}$ & No data & + & Tsutsui et al. 1997 \\
\hline $\begin{array}{l}\text { Syrian hamster embryo } \\
\text { cells }\end{array}$ & $\begin{array}{l}\text { Unscheduled DNA } \\
\text { synthesis }\end{array}$ & No data & + & Tsutsui et al. 1997 \\
\hline Human lymphocytes & $\begin{array}{l}\text { Sister chromatid } \\
\text { exchanges }\end{array}$ & + & + & $\begin{array}{l}\text { Morimoto and Wolff } \\
\text { 1980; Morimoto et al. } \\
1983\end{array}$ \\
\hline Human lymphocytes & $\begin{array}{l}\text { Sister chromatid } \\
\text { exchanges }\end{array}$ & No data & - & Jansson et al. 1986 \\
\hline Human lymphocytes & $\begin{array}{l}\text { Sister chromatid } \\
\text { exchanges }\end{array}$ & No data & + & Erexson et al. 1985 \\
\hline Human lymphocytes & DNA damage & No data & + & Li et al. 2005 \\
\hline
\end{tabular}


3. HEALTH EFFECTS

Table 3-5. Genotoxicity of Phenol In Vitro

\begin{tabular}{|c|c|c|c|c|}
\hline \multirow[b]{2}{*}{ Species (test system) } & \multirow[b]{2}{*}{ End point } & \multicolumn{2}{|c|}{ Results } & \multirow[b]{2}{*}{ Reference } \\
\hline & & $\begin{array}{l}\text { With } \\
\text { activation }\end{array}$ & $\begin{array}{l}\text { Without } \\
\text { activation }\end{array}$ & \\
\hline Human diploid fibroblasts & DNA synthesis & No data & + & Poirier et al. 1975 \\
\hline HeLa cells & DNA synthesis & + & No data & $\begin{array}{l}\text { Painter and Howard } \\
1982\end{array}$ \\
\hline
\end{tabular}

$+=$ positive response; $-=$ negative response; DNA = deoxyribonucleic acid 
test systems. Results for various end points (gene mutation, chromosomal aberration, micronuclei, DNA damage, sister chromatid exchanges, and unscheduled DNA synthesis) have been both positive and negative. The mixed results in both the in vivo and in vitro assays indicate that under certain conditions, especially at higher doses, phenol has the potential to be genotoxic. However, at the exposure levels likely to occur near hazardous waste sites, phenol is not anticipated to be genotoxic.

Positive and negative results have been reported for phenol in in vivo chromosomal aberration tests. Increases in chromosomal aberrations have been reported in bone marrow (Shelby and Witt 1995) and in spermatocytes (Bulsiewicz 1977) from mice treated with phenol. Chromosomal aberrations were reported in the bone marrow of male $\mathrm{B} 6 \mathrm{C}_{3} \mathrm{~F}_{1}$ mice exposed to phenol through intraperitoneal injection (Shelby and Witt 1995). Bulsiewicz (1977) also reported results of a five-generation study with Porton strain inbred mice. Chromosomal aberrations were monitored in spermatogonia and spermatocytes of gavage treated mice. Dose dependent increases in aberrations were observed with succeeding generations. The investigator attributed the observed chromosomal effects to interactions of phenol with the spindle apparatus. Other studies have not observed chromosomal aberrations in bone marrow from mice treated with phenol (Barale et al. 1990; Chen and Eastmond 1995a; Pashin et al. 1987). In tests of feeding and injection exposures of Drosophila to phenol, results were negative in sex-linked recessive lethal assays (Gocke et al. 1981; Sturtevant 1952).

Positive (Ciranni et al. 1988; Li et al. 2005; Shelby and Witt 1995) and negative (Barale et al. 1990; Gocke et al. 1981) results were reported for in vivo micronucleus assays in bone marrow isolated from mice treated with phenol. Bone marrow micronucleus tests were positive for male $\mathrm{B} 6 \mathrm{C} 3 \mathrm{~F}_{1}$ mice exposed to phenol through intraperitoneal injection (Shelby and Witt 1995). In a study of pregnant CD-1 mice receiving doses of phenol, maternal bone marrow micronuclei were studied. Pregnant mice were treated by gavage with a single dose of $265 \mathrm{mg} / \mathrm{kg}$ of phenol on GD 13. Observed effects included an increase in bone marrow micronuclei and cytotoxicity. Micronuclei were not observed in fetal liver tissue (Ciranni et al. 1988). Bone marrow of Kunming mice exposed to concentrations of 20, 40, or $80 \mathrm{mg} / \mathrm{kg}$ of phenol through intraperitoneal injection showed increased frequency of micronuclei at dose levels of 40 and $80 \mathrm{mg} / \mathrm{kg}$ ( $\mathrm{Li}$ et al. 2005). However, exposures of male CD-1 mice to maximum intraperitoneal injections of $160 \mathrm{mg} / \mathrm{kg}$ resulted in negative micronucleus tests (Barale et al. 1990). Results were also negative for micronucleus assays in male and female NMRI mice dosed twice by intraperitoneal injections of $188 \mathrm{mg} / \mathrm{kg}$ of phenol (Gocke et al. 1981). 
Phenol increased DNA synthesis in tubular renal and liver epithelial cells from mice (Amlacher and Rudolph 1981). Skare and Schrotel (1984) reported the results of experiments where Sprague-Dawley rats were dosed with single intraperitoneal injections of $79 \mathrm{mg} / \mathrm{kg}$ or five daily intraperitoneal injections of $39.5 \mathrm{mg} / \mathrm{kg} /$ day. Single strand breaks were not observed in testicular cells. A DNA synthesis test in male $\mathrm{B} 6 \mathrm{C} \mathrm{F}_{1}$ mice dosed by gavage with concentrations of 0,300 , and $600 \mathrm{mg} / \mathrm{kg}$ of phenol also was negative (Miyagawa et al. 1995).

In vitro tests with phenol for gene mutations in microorganisms have yielded both negative (Florin et al. 1980; Haworth et al. 1983; Kubo et al. 2002; Nagel et al. 1982; Pool and Lin 1982) and positive (Demerec et al. 1951; Gocke et al. 1981) results. Negative results have been reported in Salmonella typhimurium strains TA98, TA100, TA1535, TA1537, and TA1538 with and without S9 activation (Haworth et al. 1983; Kubo et al. 2002; Pool and Lin 1982). However, increased mutagenicity was observed in S. typhimurium TA98, with S9 activation (Gocke et al. 1981). Paschin and Bahitova (1982) also reported positive results at the HGPRT locus of V79 for mutagenicity studies involving exposures of Chinese hamster ovary (CHO) cells, with S9 activation. Studies of Syrian hamster embryos (SHE) were also positive for mutagenicity (Tsutsui et al. 1997).

In vitro studies regarding chromosomal aberrations in eukaryotic cells have been positive in Aspergillus (Crebelli et al. 1987), and in SHE cells (Tsutsui et al. 1997), and negative in mouse lymphoma cells (Pellack-Walker and Blumer 1986) and CHO cells (Sze et al. 1996). Tsutsui et al. (1997) reported dosedependent increases in the frequencies of chromosome aberrations in SHE cells exposed to phenol. Single strand breaks were not observed in mouse lymphoma L5178YS cells (Pellack-Walker and Blumer 1986).

The report of an in vitro micronucleus study with $\mathrm{CHO}$ cells was positive with and without S9 activation, with stronger results observed with S9 activation (Miller et al. 1995).

Results of in vitro sister chromatid exchange tests were reported as positive and negative. Phenol produced dose-related increases in sister chromatid exchanges in human lymphocytes at doses of $\geq 500 \mu \mathrm{M}$ (Erexson et al. 1985). In contrast, sister chromatid exchanges were not observed in human lymphocytes incubated with 0-2,000 $\mu \mathrm{M}$ phenol (Jansson et al. 1986). Incubation of human lymphocytes with $1,000,000 \mu \mathrm{M}$, but not $200,000 \mu \mathrm{M}$ phenol for 72 hours resulted in an increase in sister chromatid exchanges (Morimoto and Wolff 1980). Exposures of human lymphocytes to 3,000 $\mu \mathrm{M}$ phenol with S9 
activation also resulted in an increase in sister chromatid exchanges (Morimoto et al. 1983). Phenol induced sister chromatid exchanges in SHE cells at doses of 1,000 and 3,000 $\mu \mathrm{M}$ (Tsutsui et al. 1997).

In vitro assays for DNA synthesis have been negative in rat liver mitochondria (Schwartz et al. 1985), and positive in human fibroblasts (Poirier et al. 1975) and HeLa cells (Painter and Howard 1982). However, unscheduled DNA synthesis was induced to the same degree in SHE cells incubated with phenol in concentrations ranging from 1 to $30 \mu \mathrm{M}$ (Tsutsui et al. 1997).

An in vitro study of DNA damage using the comet assay indicated that phenol induced DNA damage in human lymphocytes, mouse spermatocytes, and crucian erythrocytes. In these tissues, amounts of DNA damage increased in conjunction with increasing doses. Observed DNA damage to mouse spermatocytes and crucian erythrocytes was more significant than DNA damage to human lymphocytes (Li et al. 2005).

\subsection{TOXICOKINETICS}

Phenol is readily absorbed and widely distributed following inhalation, oral, and dermal exposure. The distribution of phenol is thought to be dependent on blood flow. Conjugates with glucuronic acid and sulfate are the major metabolites of phenol, although small amounts of the hydroxylation products catechol and hydroquinone are also produced. Sulfotransferase and glucuronyltransferases are present in most tissues, although the major sites of phenol conjugation are the gastrointestinal tract, liver, lung, and kidney. Because of the large capacity of the intestines and liver to conjugate phenol, the fact that the first-pass effect occurs following oral exposure but not following dermal exposure may contribute to the greater potential for phenol to result in adverse effects following dermal exposure. Phenol and its conjugates are predominantly excreted in the urine.

\subsubsection{Absorption}

\subsubsection{Inhalation Exposure}

Phenol is absorbed readily after inhalation exposure. Eight subjects were exposed to phenol vapors (1.6$5.2 \mathrm{ppm}$ ) for 8 hours (Piotrowski 1971). Subjects were exposed through a face mask in order to eliminate the possibility of percutaneous absorption. The concentration of phenol in inhaled and exhaled air was determined, and urine was analyzed for total phenol (phenol and phenol conjugates). Steady-state appeared to be achieved within 3 hours after initiating exposure; steady-state retention was $60-88 \%$. 
Urinary recovery of phenol that had been retained in the lungs was $99 \pm 8 \%$ within 24 hours after initiating exposure.

Total urinary phenol was determined at about 7 hours into an 8-hour shift in Bakelite workers exposed to airborne phenol at 0.16-32 ppm (Ohtsuji and Ikeda 1972). Daily urinary excretion of total phenol was $99 \%$ of the estimated amount inhaled indicating that phenol is readily absorbed. However, lung retention was not measured, and the contribution of percutaneous absorption to urinary phenol could not be evaluated in this study.

Rats exposed by intratracheal instillation to $\left[{ }^{14} \mathrm{C}\right]$-labeled phenol also demonstrated rapid absorption kinetics, with most of the radioactivity being excreted within 72 hours (Hughes and Hall 1995). Rats exposed for 6 hours nose-only to $25 \mathrm{ppm}\left[{ }^{14} \mathrm{C}\right]$-labeled phenol demonstrated rapid absorption. Greater than $90 \%$ of phenol-derived radioactivity was measured in the urine 30 minutes after initiation of exposure (Hiser et al. 1994).

\subsubsection{Oral Exposure}

Based on the rapid excretion of phenol and its metabolites in urine, it has been concluded that phenol is readily absorbed by the oral route in humans (Capel et al. 1972) and a variety of mammalian species including monkeys (Capel et al. 1972), rodents (Capel et al. 1972; Edwards et al. 1986; Hughes and Hall 1995; Kao et al. 1979; Kenyon et al. 1995), dogs (Capel et al. 1972), rabbits (Capel et al. 1972), cats (Capel et al. 1972; French et al. 1974), and pigs (Capel et al. 1972; Kao et al. 1979).

In three men given a single oral dose of $0.01 \mathrm{mg} / \mathrm{kg}\left[{ }^{14} \mathrm{C}\right]$-labeled phenol in food or drink, about $90 \%$ (range 85-98\%) of the dose was excreted in the urine in 14 hours (Capel et al. 1972). In this same study, urinary recovery of orally administered $\left[{ }^{14} \mathrm{C}\right]$-labeled phenol was determined in 18 other mammalian species; mean 24-hour recoveries of ${ }^{14} \mathrm{C}$ ranged from $95 \%$ in the rat to $31 \%$ in the squirrel monkey. Rats exposed orally to radiolabeled phenol demonstrated rapid absorption and excretion, with most of the radioactivity being excreted within 72 hours (Hughes and Hall 1995). The gastrointestinal absorption of phenol has also been studied in rats with in situ preparations. The absorption kinetics of $\left[{ }^{14} \mathrm{C}\right]$-labeled phenol administered directly into the small intestines of rats were described as first-order, with a rate constant for intestinal absorption of $0.127 \pm 0.003$ minute $^{-1}$ (or half-life of $5.5 \pm 0.5$ minutes from $\mathrm{t}^{1} / 2=0.693 \mathrm{k}$ ) (Humphrey et al. 1980). Two hours after $\left[{ }^{14} \mathrm{C}\right]$-labeled phenol was injected into the small 
intestines of anesthetized rats, recoveries in the urine were $77.9 \pm 2 \%$ after a $12.5-\mathrm{mg} / \mathrm{kg}$ dose, and $76.9 \pm 5.8 \%$ after a $25-\mathrm{mg} / \mathrm{kg}$ dose (Kao et al. 1979).

Hiser et al. (1994) reported results of the kinetics of oral doses of phenol in rats. Exposures included single and multiple bolus and drinking water doses of different concentrations. Peak blood concentrations of free phenol of $0.02 \mu \mathrm{g} / \mathrm{g}$ blood in rats receiving $1.5 \mathrm{mg} / \mathrm{kg}$ bolus dose were reached $1-3$ minutes after receiving the dose. A terminal half-life of about 8 minutes was calculated for phenol following low doses. Peak blood concentrations of phenol of $46.4 \mu \mathrm{g} / \mathrm{g}$ blood in rats receiving $150 \mathrm{mg} / \mathrm{kg}$ bolus dose were reached 1 minute after receiving the dose. Since the doses were separated by only a factor of 100 and the blood peak concentration by a factor of 2,320 , these results suggest saturated absorption or saturated metabolism and excretion. After 24 hours, concentrations of administered $\left[{ }^{14} \mathrm{C}\right]$-radioactivity were $>90 \%$ in the urine, regardless of the dosing method or concentration.

\subsubsection{Dermal Exposure}

Phenol is readily absorbed through the skin, and the skin is considered the primary route of entry during occupational exposure (ACGIH 2005). Whole-body skin exposures studies were conducted in volunteers lightly clothed and unclothed (Piotrowski 1971). The subjects were exposed to phenol vapor (35\% humidity, $26^{\circ} \mathrm{C}$ ) at concentrations of $1.3,2.6$, or $6.5 \mathrm{ppm}$ for 6 hours. Fresh air was supplied to the subjects through a face mask in order to prevent absorption of phenol through the lungs. The total amount of phenol excreted in urine during and after exposure (minus baseline excretion) was used as a measure of absorption. Absorption increased proportionately with exposure level. Percutaneous clearance ( $\mathrm{mg}$ phenol absorbed through the skin per hour/mg phenol per $\mathrm{m}^{3}$ of air) was estimated to be $0.35 \mathrm{~m}^{3} /$ hour. Thus, an amount of phenol equivalent to that contained in $0.35 \mathrm{~m}^{3}$ of air was absorbed through the skin each hour.

The data reported by Piotrowski (1971) provide a basis for comparing the relative contributions of lung and percutaneous absorption during exposures to phenol vapor. Assuming a ventilation rate for the human of $0.8 \mathrm{~m}^{3} /$ hour (EPA 1986a) and a steady-state lung retention of inhaled phenol of $0.7 \mathrm{~m}^{3} /$ hour (Piotrowski 1971), clearance of airborne phenol through the lung is $\approx 0.6 \mathrm{~m}^{3} /$ hour. Thus, an amount of phenol equivalent to that contained in $0.6 \mathrm{~m}^{3}$ of air was absorbed through the lungs each hour. It can be concluded that at any given exposure level within the range of $5-25 \mathrm{mg} / \mathrm{m}^{3}(1.3-6.4 \mathrm{ppm})$, percutaneous absorption $\left(0.35 \mathrm{~m}^{3} /\right.$ hour $)$ will be about half that of absorption through the lungs $\left(0.6 \mathrm{~m}^{3} /\right.$ hour $)$. 
Percutaneous absorption of phenol applied in solution directly to the forearm $\left(15.6 \mathrm{~cm}^{2}\right)$ of volunteers has been measured (Baranowska-Dutkiewicz 1981). Absorption rate from a 2-mL reservoir of an aqueous phenol solution $(2.5,5.0$, or $10.0 \mathrm{~g} / \mathrm{L})$ was constant for 60 minutes $\left(0.08 \mathrm{mg} / \mathrm{cm}^{2} /\right.$ hour $)$ and increased proportionately with applied concentration. Approximately $13 \%$ of the applied dose was absorbed in 30 minutes, of which $80 \%$ (range 58-98\%) was recovered in the urine within 24 hours.

When human skin was treated in vitro with $0.0013-0.0027 \mathrm{mg} / \mathrm{cm}^{2}\left[{ }^{14} \mathrm{C}\right]-$ labeled phenol and left unoccluded, $20 \%$ of the radioactivity was absorbed when analyzed 72 hours later, while $7 \%$ remained on the skin surface (Hotchkiss et al. 1992). Covering the skin with a teflon cap resulted in the absorption of $47 \%$, with $3 \%$ recovered in the skin. When rat skin was subjected to the same exposure regime in this study, 72 hours later, $24 \%$ of the radioactivity was absorbed with $22 \%$ recovered in the skin when the skin was unoccluded, and $36 \%$ was absorbed with 3-4\% recovered in the skin when the skin was occluded.

In rats in which a $0.03-\mathrm{mg} / \mathrm{kg}$ dose of $\left[{ }^{14} \mathrm{C}\right]$-labeled phenol was placed on the skin, only $1-5 \%$ of the dose remained in the body 72 hours later (Hughes and Hall 1995). The dermal absorption of phenol was also studied in three pigs in which undiluted phenol was placed on the skin for 1 minute, and the peak plasma level was determined (Pullin et al. 1978). Plasma levels were not measurable in one pig treated with a dose of $90 \mathrm{mg} / \mathrm{kg}$ over a surface area of $91.6 \mathrm{~cm}^{2}$. In pigs treated with a dose of $500 \mathrm{mg} / \mathrm{kg}$ over surface areas of 91.6 and $1,135.5 \mathrm{~cm}^{2}$, peak plasma levels of 0.9 and $30.5 \mathrm{ppm}$ were reported, respectively.

Permeability coefficients for phenol in isolated skin patches from nude mice have been determined (Behl et al. 1983). The permeability coefficient increased as the concentration of the applied aqueous phenol solution increased; doubling the concentration from 20 to $40 \mathrm{~g} / \mathrm{L}$ resulted in a 12-fold increase in mean permeability coefficient $(0.007-0.085 \mathrm{~cm} /$ hour $)$. The value obtained for the permeability coefficient when $60 \mathrm{~g} / \mathrm{L}$ was applied to the skin patch $(0.169 \mathrm{~cm} /$ hour $)$ was similar to that obtained for skin patches in which the stratum corneum had been removed. It was concluded that phenol concentrations exceeding $20 \mathrm{~g} / \mathrm{L}$ may destroy a diffusion barrier normally provided by the intact stratum corneum, permitting increased percutaneous absorption.

Dermal absorption of phenol in the presence of various types of soil was measured in vitro using skin patches from pigs (Skowronski et al. 1994). Maximum phenol penetration occurred between 2 and 4 hours after treatment in all cases. Compared to samples with no soil present, the presence of sandy soil reduced the peak penetration by one-half, and the presence of clay soil reduced peak penetration by twothirds. 


\subsubsection{Other Routes of Exposure}

Nomoto et al. (1987) studied the absorption of phenol after injection into 20 patients as part of lumbar or thoracic sympathetic blockades. Patients were injected with 5-10 mL 7\% phenol. The concentrations of unconjugated and conjugated phenol (sulfate esters and glucuronic esters) were monitored in blood and urine samples. Unconjugated phenol reached a mean peak concentration in blood of $3.01 \pm 0.28 \mu \mathrm{g} / \mathrm{mL}$ $18.8 \pm 2.5$ minutes after the injection. The mean peak concentration of conjugated phenol in the blood was $4.15 \pm 0.25 \mu \mathrm{g} / \mathrm{mL} 54.9 \pm 4.5$ minutes after the injection. The authors concluded that uptake times indicated rapid absorption of phenol after injection. The lag time of unconjugated phenol was $5.3 \pm 1.6$ minutes, indicating that phenol remained at the injection site before being taken up into the blood. The lag time of conjugated phenol was $9.9 \pm 5.9$ minutes.

There is also indirect evidence of phenol being absorbed following phenol injection sclerotherapy for hemorrhoids. Suppiah and Perry (2005) reported the case of a 43-year-old man who developed jaundice after an unspecified number of injections of $2 \mathrm{~mL}$ of a $5 \%$ solution of phenol at hemorrhoidal tissue during several months. Liver function tests returned to normal after 6 months.

\subsubsection{Distribution}

\subsubsection{Inhalation Exposure}

No studies were found regarding tissue distribution of phenol in humans after inhalation exposure.

Rats exposed by intratracheal instillation to radiolabeled phenol were sacrificed 72 hours later and analyzed for tissue distribution of the radioactivity (Hughes and Hall 1995). Of the radioactivity remaining in the body $(1-5 \%)$, a majority was distributed in the lungs $(0.13 \%)$, skin $(0.13 \%)$, blood $(0.07 \%)$, muscle $(0.03 \%)$, fat $(0.02 \%)$, and liver $(0.02 \%)$.

No information was found on the placental transfer and distribution of phenol; however, Ghantous and Danielsson (1986) examined this question for benzene, the principal metabolite of which is phenol. Mice, at GDs 11, 14, and 17, were exposed by inhalation to $\left[{ }^{14} \mathrm{C}\right]$-benzene and the distribution of benzene and its volatile and nonvolatile metabolites was examined using whole-body autoradiography and assessment of tissue concentrations of ${ }^{14} \mathrm{C}$ (day 17 only). The authors indicated that the exposure regimen $(50 \mu \mathrm{Ci}$ of $\left[{ }^{14} \mathrm{C}\right]$-benzene in maize oil, volatilized by gentle heating) would theoretically produce $2,000 \mathrm{ppm}$ in the 
inhalation chamber. Measurements of the difference between the amount added to the chamber and the amount inhaled by the animals indicated an uptake of $90 \%$ (i.e., $45 \mu \mathrm{Ci}$ ). These authors did not specifically characterize the metabolites, but were able to show that the $\left[{ }^{14} \mathrm{C}\right]$-labeled volatile and nonvolatile activity crossed the placental barrier. There was no evidence of preferential accumulation. Indeed, the concentration of volatile and nonvolatile radioactivity in fetal tissues was much lower than that observed in the corresponding maternal tissues. As a metric of the relative accumulation, the authors noted that compared to maternal brain tissue, fetal uptake of benzene was only $8 \%$.

In a study conducted by Hiser et al. (1994), rats were exposed 6 hours nose-only to $25 \mathrm{ppm}\left[{ }^{14} \mathrm{C}\right]$-phenol for either 1 or 8 days. Mean percent of administered dose/g were reported for several tissues. Radioactivity was quantified in blood, bone, brain, fat, heart, kidney, liver, lung, muscle, skin, spleen, testes, ovaries, and carcass 24 hours after exposure. No single tissue seemed to preferentially accumulate phenol-derived radioactivity and, in all cases, concentrations were $<0.02 \%$ administered dose $/ \mathrm{g}$ tissue. No significant differences were seen between the 1- and 8-day exposure experiments.

\subsubsection{Oral Exposure}

Limited information in humans is available from cases of accidental or intentional ingestion of phenol that resulted in fatalities. One case involved ingestion of a mixture of phenol and cresol. The concentrations of phenol measured in the blood, urine, and stomach content were 58.3, 3.3, and $115 \mu \mathrm{g} / \mathrm{mL}$, respectively (Boatto et al. 2004). Another case involved ingestion of a mixture of phenol and chloroform. Phenol was measured at $60 \mu \mathrm{g} / \mathrm{mL}$ in the blood and $208 \mu \mathrm{g} / \mathrm{mL}$ in the urine. Concentrations in the brain, lungs, liver, and kidney were 106, 116, 166, and $874 \mu \mathrm{g} / \mathrm{g}$, respectively (Tanaka et al. 1998). The third case involved ingestion of phenol, which resulted in the following tissue concentrations of phenol: blood, $130 \mu \mathrm{g} / \mathrm{mL}$; urine, $47 \mu \mathrm{g} / \mathrm{mL}$; bile, $187 \mu \mathrm{g} / \mathrm{mL}$; brain, $486 \mu \mathrm{g} / \mathrm{g}$; kidney, $331 \mu \mathrm{g} / \mathrm{g}$; muscle, $204 \mu \mathrm{g} / \mathrm{g}$; liver, $228 \mu \mathrm{g} / \mathrm{g}$; and stomach content, $668 \mathrm{mg}$ (Lo Dico et al. 1989).

In animals, information is available for rabbits (Deichmann 1944) and rats (Hiser et al. 1994; Hughes and Hall 1995; Liao and Oehme 1981). In rabbits, distribution is rapid, with peak tissue concentrations achieved in most tissues within 1 hour after dosing. The highest peak concentrations and fraction of administered dose are found in the liver; $>90 \%$ of the administered dose is eliminated from tissues within 24 hours. 
The levels of phenol in various tissues of five rabbits given a lethal $\left(\mathrm{LD}_{50}\right)$ oral dose of phenol $(500 \mathrm{mg} / \mathrm{kg}$ ) were determined (Deichmann 1944). The rabbits were killed within 1-3 minutes after dosing when twitching, the first sign of systemic toxicity, appeared. The highest concentrations of total phenol (free plus conjugates) were found in the liver (20.9-30.4 mg/100 g tissue), lungs (5.1$17.1 \mathrm{mg} / 100 \mathrm{~g})$, blood (6.1-12.6 mg/100 g), brain and spinal cord (3.1-10.4 mg/100 g), and kidneys (2.3$7.1 \mathrm{mg} / 100 \mathrm{~g})$.

The kinetics of tissue distribution of $\left[{ }^{14} \mathrm{C}\right]$-labeled phenol in rats given $207 \mathrm{mg} / \mathrm{kg}$ of $\left[{ }^{14} \mathrm{C}\right]-$ labeled phenol, a sublethal $\left(\approx 0.5 \times \mathrm{XD}_{50}\right)$ oral dose, were studied (Liao and Oehme 1981). Although all rats survived for 16 hours, signs of systemic toxicity were observed including twitching of muscles around the eyes and ears, convulsions, and coma persisting for 15-30 minutes. Thirty minutes after dosing, $28.4 \%$ of administered ${ }^{14} \mathrm{C}$ was recovered in tissues (liver, kidney, adrenal, thyroid, spleen, blood, lung, thymus, brain, testes, heart, muscle, and fat). Sixteen hours after dosing, $0.3 \%$ of the administered dose was recovered in tissues. Concentrations of ${ }^{14} \mathrm{C}$ were highest in all tissues 30 minutes after dosing, with the exception of the thyroid gland, in which peak concentrations were achieved after 2 hours. The highest concentration and fraction of administered dose were found in the liver; $42 \%$ (range 29-56\%) of the administered dose was recovered in the liver 30 minutes after dosing. Approximately $67-85 \%$ of the ${ }^{14} \mathrm{C}$ in blood was present in the plasma fraction, of which $41-50 \%$ was bound to plasma proteins or other macromolecules. The elimination half-time for ${ }^{14} \mathrm{C}$ was $<4$ hours. Based on their results, the study authors suggested that blood flow determines the tissue uptake of the radiolabel from phenol.

Rats exposed orally to radiolabeled phenol were sacrificed 72 hours later and analyzed for tissue distribution of the radioactivity (Hughes and Hall 1995). Of the radioactivity remaining in the body, a majority was distributed in the muscle $(0.08 \%)$, skin $(0.07 \%)$, fat $(0.02 \%)$, liver $(0.02 \%)$, and blood $(0.02 \%)$.

No evidence of exposure-related DNA adduct formation in femur bone marrow, Zymbal gland, liver, or spleen was seen in rats treated orally with $75 \mathrm{mg} / \mathrm{kg} /$ day phenol for 4 days (Reddy et al. 1990). In this study, concurrent in vitro exposures of these tissues did produce adducts, suggesting that efficient detoxification and excretion mechanisms may be operating in vivo.

In a study conducted by Hiser et al. (1994), rats were exposed to $\left[{ }^{14} \mathrm{C}\right]$-phenol via single gavage doses of $1.5,15$, or $150 \mathrm{mg} / \mathrm{kg}$, multiple gavage doses of $1.5 \mathrm{mg} / \mathrm{kg} /$ day, $5,000 \mathrm{ppm}$ in drinking water for 1 day, or 5,000 ppm in drinking water for 8 days. In all cases, phenol-derived radioactivity was detected in blood, 
bone, brain, fat, heart, kidney, liver, lungs, skin, spleen, testes, ovaries, and carcass 24 hours after exposures. Regardless of the dosing method or dose level, no single tissue seemed to accumulate radioactivity, with all measured concentrations being $<0.02 \%$ of the administered dose $/ g$ tissue.

\subsubsection{Dermal Exposure}

Limited information is available in humans from a fatal case. Tissue samples (liver, blood, lung, urine, and stomach contents) from an individual who was painted with benzyl benzoate with a brush that had been soaked in $80 \%$ phenol were analyzed for phenol. The blood contained $4.7 \mu \mathrm{g} / \mathrm{mL}$ phenol and unhydrolyzed and hydrolyzed liver samples contained 3.3 and $7.1 \mu \mathrm{g} / \mathrm{g}$ phenol, respectively. Phenol was not detected in the lung, urine, or stomach contents (Lewin and Cleary 1982).

Rats exposed dermally to radiolabeled phenol were sacrificed 72 hours later and analyzed for tissue distribution of the radioactivity (Hughes and Hall 1995). Of the radioactivity remaining in the body (1$5 \%)$, a majority was distributed in the skin $(0.021 \%)$, muscle $(0.02 \%)$, fat $(0.03 \%)$, liver $(0.01 \%)$, and blood $(0.02 \%)$.

\subsubsection{Other Routes of Exposure}

No studies were located regarding distribution of phenol in humans after exposure by other routes.

Microdialysis sampling has been used in rats infused with phenol $(0.181 \mathrm{nmol} /$ minute for 90 minutes $)$ to study excretion into the bile (Scott and Lunte 1993). For all phenol metabolites, bile concentrations were higher than liver concentrations indicating that the metabolites are actively excreted in the bile.

The distribution of phenol in the liver has been studied in mice treated intravenously with $31.4 \mathrm{mg} / \mathrm{kg}$ phenol (Davies and Lunte 1996). Microdialysis probes used to monitor the distribution of phenol metabolites in three regions of the liver (anterior, median, posterior) indicated that phenol-glucuronide was the most prevalent metabolite in all three regions, but the level was significantly lower in the anterior region compared to the other regions. When phenol was delivered to the liver through microdialysis probes, no regional differences in the delivery of phenol or metabolite formation were observed, indicating that clearance of phenol from the liver is dominated by blood flow rather than metabolism. 


\subsubsection{Metabolism}

Figure 3-3 shows the general metabolic pathways that transform phenol prior to its excretion in the urine. Three different enzymes systems catalyze the reactions that transform phenol. Cytosolic phenol sulfotransferases catalyze the transfer of inorganic sulfur from the activated 3'-phosphoadenosine-5'phosphosulfate donor molecule to the hydroxyl group on phenol. Microsomal membrane-located uridine diphosphate (UDP) glucuronosyltransferases catalyze the transfer of an activated glucuronic acid molecule to the hydroxyl moiety of phenol to form an O-glucuronide conjugate. Cytochrome P4502E1, also microsomally located, catalyzes the hydroxylation of phenol to form hydroquinone (and to a much lesser extent, catechol), which is then acted upon by the phase II enzymes (Buxton 2006; Campbell et al. 1987; Gut et al. 1996; Koop et al. 1989; McFadden 1996; Powley and Carlson 2001; Snyder et al. 1993). Hydroquinone can, in turn, form conjugates, undergo peroxidation to form benzoquinone, or undergo further oxidation to form trihydroxybenzene. All three enzyme systems that metabolize phenol are found in multiple tissues and there is competition among them not only for phenol, but also for subsequent oxidative products, like hydroquinone. As a consequence, the relative amount of the products formed can vary based on species, dose and route of administration.

Cytochromes other than CYP2E1 also seem to be involved in the metabolism of phenol as demonstrated by Powley and Carlson (2001) in experiments utilizing chemical inhibitors of CYP2E1, CYP2B, CYP2F2, and CYP2E1 knockout mice. The investigators found that CYP2E1 was responsible for only approximately $50 \%$ of phenol metabolism in liver, suggesting the participation of other cytochromes. Experiments in pulmonary microsomes showed that both CYP2E1 and CYP2F2 played important roles in the metabolism of phenol.

Phenol can also undergo peroxidation to form 4,4'-biphenol and diphenoquinone. This has been demonstrated in studies that used in vitro cell preparations with high peroxidase activity (Eastmond et al. 1986; Post et al. 1986), purified peroxidase enzymes (Smart and Zannoni 1984; Subrahmanyam and O'Brien 1985), or cell lines that have high myeloperoxidase activity (Kolachana et al. 1993). Thus far, there is no direct evidence that these peroxidation reactions occur in vivo.

In vivo, the gastrointestinal tract, liver, lung, and kidney appear to be the major sites of phenol sulfate and glucuronide conjugation of simple phenols (Cassidy and Houston 1984; Powell et al. 1974; Quebbemann and Anders 1973; Tremaine et al. 1984). Experiments conducted by Cassidy and Houston (1984) in rats injected intra-arterially, intravenously, or intraduodenally (doses ranged from 0.5 to $15 \mathrm{mg} / \mathrm{kg}$ ) allowed 


\section{Figure 3-3. Metabolism of Phenol}

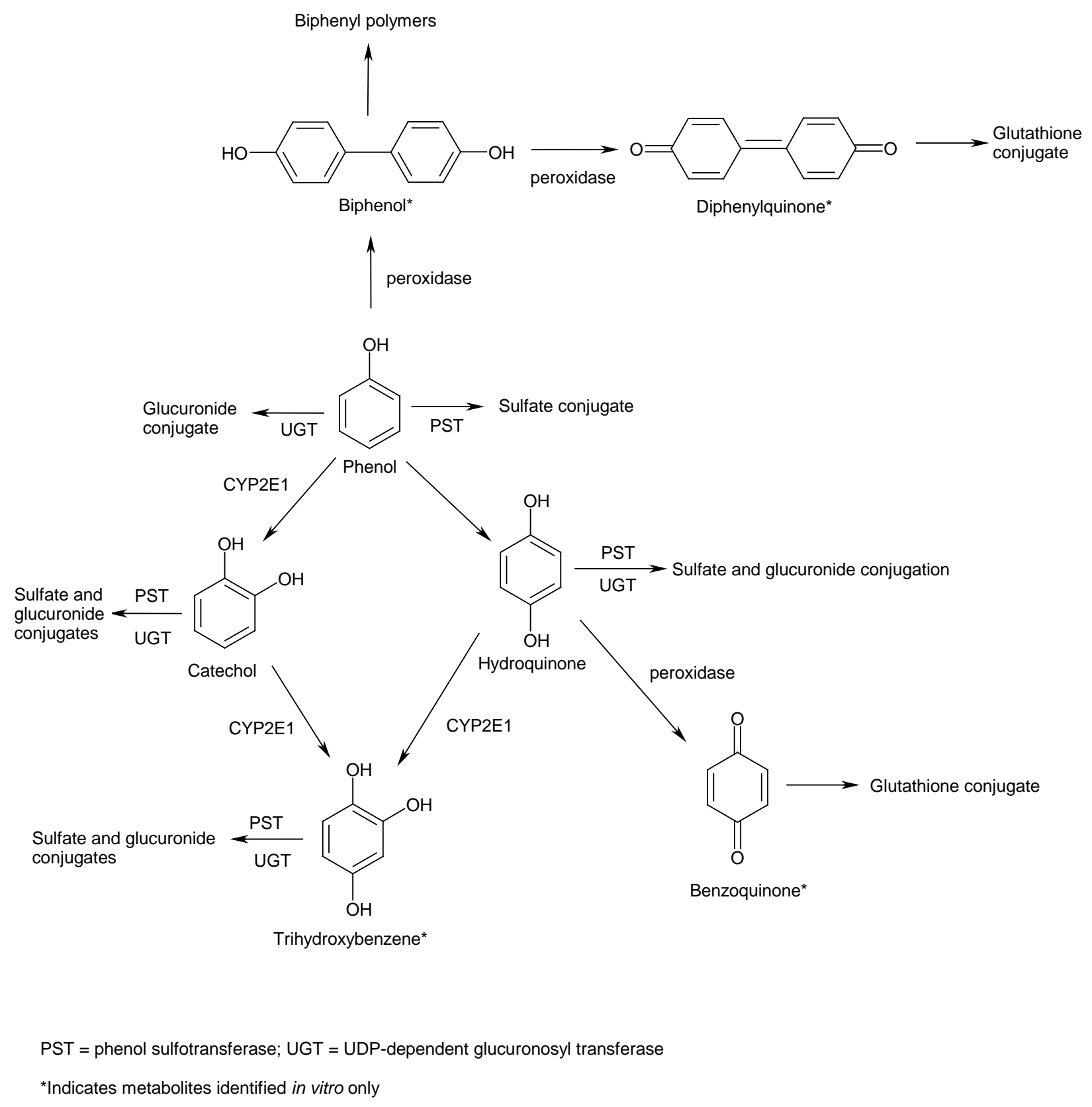

Source: EPA 2002 
them to evaluate the first-pass metabolism by different tissues. It was assumed that phenol that was systemically available had not been conjugated or metabolized and, therefore, the doses at which this occurred reflected the doses at which metabolic reactions were saturated. The investigators found that metabolism became nearly saturated in the liver at doses 10 times lower than in the endothelial lung, whereas metabolism in the gut was not saturated even at the highest dose tested. They also observed that the endothelial lung had a much lower affinity for phenol than the liver and gut. However, caution should be exercised when interpreting the results of the metabolic capacity of the lung because normal exposure results in exposure of the epithelial respiratory tract rather than the endothelial surface, as occurred in this study.

Four principal metabolites have been identified in mammals: two phenol and two hydroquinone conjugates (of sulfate and glucuronide) (Capel et al. 1972; Hoffmann et al. 1999; Kenyon et al. 1995; Wheldrake et al. 1978). In humans, rats, and mice given low doses of phenol orally, sulfate conjugates of phenol were found to predominate. However, in guinea pigs, pigs, and fruit bats, the glucuronide conjugates were dominant (Capel et al. 1972). In humans given an oral dose of $0.01 \mathrm{mg} / \mathrm{kg}, 77 \%$ of the urinary ${ }^{14} \mathrm{C}$ was identified as phenyl sulfate, $16 \%$ as phenyl glucuronide, and trace amounts $(<1 \%)$ as the sulfate and glucuronide conjugates of hydroquinone (Capel et al. 1972).

In mice, phenyl sulfate was the predominant urinary metabolite for low doses $(1-21 \mathrm{mg} / \mathrm{kg})$ of phenol administered either by gavage and intravenously; however, as the dose increased, a decrease in phenol sulfation and a concomitant increase in glucuronidation of both phenol and hydroquinone was seen suggesting saturation of the sulfation pathway (Kenyon et al. 1995). The degree of saturation appeared to be slightly greater following gavage administration, and intravenous administration resulted in higher proportions of the products of oxidative metabolism, with male mice being more prone than female mice. These latter observations suggest that the oxidative pathway become more prominent when phenol is introduced directly into the circulation, bypassing an initial intestinal sulfate conjugation process, and also suggests that the sulfate conjugation process saturates at a lower concentration in males than in females.

Similarly, in the rat, the ratio of phenyl sulfate/glucuronide conjugates in urine decreased from 2.6 to 0.7 when the intravenous dose level is increased from 1.2 to $25 \mathrm{mg} / \mathrm{kg}$ (Weitering et al. 1979). This phenomenon appears to be, at least in part, the result of differences in $\mathrm{K}_{\mathrm{m}}$ in the two pathways, in relation to their respective $\mathrm{V}_{\max }$ (Koster et al. 1981; Weitering et al. 1979). The range of substrate concentrations over which the reaction rate remains a linear function of concentration narrows as $V_{\max } / K_{m}$ decreases. A shift toward glucuronide formation as a function of dose would be expected in $V_{\max } / K_{m}$, for the sulfation 
pathway was less significant than the glucuronide pathway. Treatment of rats with an intraperitoneal dose of phenol (23-188 mg/kg) has also been shown to result in dose-dependent decreases in hepatic 3'-phosphoadeonsine 5'-phosphosulfate (PAPS), the co-substrate for the sulfate conjugation of phenol, as well as sulfate (Kim et al. 1995). The depletion of PAPS may also contribute to the saturation of sulfation at high doses of phenol. In another study of rats exposed to single and multiple bolus and drinking water doses of different concentrations, metabolites in the urine were primarily conjugates of phenol, showing dose-dependant concentrations (Hiser et al. 1994). For bolus doses, the ratios of glucuronide to sulfate phenol conjugates were 0.61 for 1.5 and $15 \mathrm{mg} / \mathrm{kg}$ doses and 1.16 for $150 \mathrm{mg} / \mathrm{kg}$ doses. Drinking water exposures resulted in a similar ratio of glucuronide to sulfate as observed for the $150 \mathrm{mg} / \mathrm{kg}$ bolus dose, with a ratio of 0.60 . For inhalation exposures, ratios ranged from 0.24 to 0.39 . Small amounts of an unidentified metabolite ( $2-4 \%$ total urinary radioactivity) were also detected.

All three enzyme systems involved in phenol metabolism have other substrates, which can competitively inhibit the metabolism of phenol, thereby changing the balance among metabolites. Inhibition of phenol sulfotransferase with chlorinated phenols (e.g., pentachlorophenol) results in increased glucuronide conjugation of simple phenols (Mulder and Scholtens 1977). Similarly, benzene is metabolized by CYP2E1; thus, high exposures to benzene may competitively inhibit phenol metabolism, resulting in decreasing hydroquinone production (and its corresponding sulfate and glucuronide conjugates) (Medinsky et al. 1995; Schlosser et al. 1993). Further information regarding the shift between sulfation, glucuronidation, and oxidation reactions is presented in Section 3.5.1, Pharmacokinetics Mechanisms.

Age- and sex-related changes in phenol sulfoconjugation were studied in hepatic cytosolic preparations from fetal, newborn, and adult rats (Iwasaki et al. 1993). Phenol sulfoconjugation activity was higher in adult males $(1.94 \pm 0.1 \mathrm{nmol} / \mathrm{mg} /$ minute) than females $(1.07 \pm 0.03 \mathrm{nmol} / \mathrm{mg} /$ minute $)$, although there were no sex-related differences in the younger rats. Activity in fetal rats was very low $(0.04 \pm 0.01 \mathrm{nmol} / \mathrm{mg} / \mathrm{minute})$. Activity at 2 days after birth was half that in adult females and a quarter of that in adult males, and remained constant until 25 days after birth. At 2 years of age, activity was intermediate between young adult male and female activities, and there were no sex-related differences. Heaton and Renwick (1991) found that young rats have a higher production of oxidative metabolism than adult rats. If this were the case in humans, children might be potentially more sensitive to the systemic effects of phenol, if a reactive intermediate is responsible for phenol toxicity. However, since glucuronidation does not appear to be limited in the young, production of oxidative products may be a smaller risk than anticipated. Caution should be exercised when extrapolating from adolescent rats to 
children, since rodents are well known to undergo a number of changes in xenobiotic-metabolizing enzymes during sexual development (Waxman et al. 1985).

\subsubsection{Elimination and Excretion}

Phenol, in its free and conjugated forms, is a normal constituent of human urine. Piotrowski (1971) reported $8.7 \pm 2.0 \mathrm{mg} /$ day as the daily excretion rate of total phenol (free plus conjugates) in human subjects with no known exposure to phenol. Others have reported a range of values. In a study of workers employed in the distillation of high-temperature phenolic fractions of tar, mean values of phenol in the urine of $13.8 \mathrm{mg} / \mathrm{L}$ in 26 male non-exposed workers and $67.8 \mathrm{mg} / \mathrm{L}$ in 89 exposed workers were reported (Bieniek 1994). The highest concentration was found 2 hours after the end of the work shift. Quint et al. (1998) evaluated the urinary phenol concentration before and after using phenol to chemically cauterize the lesion created by excision of chrondroblastoma in 11 patients. Preoperatively, the average urinary concentration of phenol was $5.1 \mathrm{mg} / \mathrm{L}$. Ling and Hanninen (1991) studied the effect of phenol on serum and urinary concentrations in patients who switched from a conventional diet to an uncooked "vegan" diet. Patients were tested at week 0, were on the vegan diet for 4 weeks, and then were on the regular diet for the second month. Urinary and serum levels of phenol were measured at weeks $0,2,4$, and 9. A significant decrease in both urinary and serum concentrations of phenol was seen within 2 weeks of adopting the vegan diet. At 2 weeks, the serum concentration had dropped from about 0.75 to $0.5 \mathrm{mg} / \mathrm{L}$ (about 30\%), and levels in urine had dropped from about $7 \mathrm{mg} / \mathrm{L}$ to about $3 \mathrm{mg} / \mathrm{L}$ (about $60 \%$ ). These data indicate that phenol is a natural product of metabolism that may vary significantly depending at least on diet, but probably also due to other factors.

Horch et al. (1994) make the statement "urine phenol concentrations should be monitored in exposed persons to determine if they are within normal range $(0.5-81 \mathrm{mg} / \mathrm{L})$," but they provide no citation for the range given. It should be noted that as late as 1980, gas chromatographic analyses of urine used to determine phenol levels showed fairly large interlaboratory variation (Van Roosmalen et al. 1981). Thus, the range of values given above, if derived from multiple references including the older literature, may be artificially broad.

\subsubsection{Inhalation Exposure}

Phenol absorbed through the lungs is excreted rapidly in urine in its free and conjugated forms. Within 24 hours after human subjects inhaled phenol at concentrations of $6-20 \mathrm{mg} / \mathrm{m}^{3}(1.5-5.1 \mathrm{ppm}), 99 \pm 8 \%$ of the phenol retained in the lungs was excreted (Piotrowski 1971). The urinary excretion of phenol was 
studied in 106 men occupationally exposed to phenol, cresols, xylenols, and other phenolic derivatives, and 26 unexposed controls (Bieniek 1994). Urine samples were taken after 4 hours at work, and in 16 workers every 2 hours for 24 hours after an 8-hour shift. The mean level of phenol in urine of the exposed workers was $87.3 \mathrm{mg} / \mathrm{L}$, compared to $11.7 \mathrm{mg} / \mathrm{L}$ in controls. The highest phenol concentrations were recorded between 8 and 10 hours after the beginning of the exposure. Exposure concentrations were not reported in this study.

A study of workers in a Bakelite factory reported a linear correlation between concentrations of phenol in the air (up to $12.5 \mathrm{mg} / \mathrm{m}^{3}$ or $3.25 \mathrm{ppm}$ ) and urinary excretion of total phenol (free plus conjugated) (Ohtsuji and Ikeda 1972). However, the urinary concentration of free phenol seemed to be independent of the environmental phenol, suggesting that under the exposure conditions, the maximum capacity to conjugate phenol had not been reached.

Urinary excretion of total phenol (free and conjugates) is considered a biomarker of exposure for phenol. The biological exposure index (BEI) for phenol, for exposure to $5 \mathrm{ppm}$ in air, is $250 \mathrm{mg} / \mathrm{g}$ creatinine when measured at the end of the shift (ACGIH 2001).

In rats exposed by intratracheal instillation to radiolabeled phenol, elimination was $95 \%$ complete after 72 hours, with the primary elimination route being through the urine (Hughes and Hall 1995). Fecal elimination was slower and accounted for less overall.

In a study conducted by Hiser et al. (1994), rats were exposed via nose-only inhalation for 6 hours to $25 \mathrm{ppm}{ }^{14} \mathrm{C}$-phenol for either 1 or 8 days. Thirty hours after initiation of exposure, mean percentages of phenol-derived radioactivity were measured in urine and feces. In rats exposed for 1 day, values in urine were $94.48 \%$ (males) and $90.92 \%$ (females). In rats exposed for 8 days, values in urine were $97.40 \%$ (males). In rats exposed for 1 day, values in feces were 3.33\% (males) and $2.02 \%$ (females). In rats exposed for 8 days, values in feces were $0.81 \%$ (males). Less than $1 \%$ remained in tissues and carcass. These results indicate rapid elimination of phenol in urine after inhalation exposure.

\subsubsection{Oral Exposure}

Phenol absorbed from the gastrointestinal tract is excreted rapidly in urine as free phenol or conjugates (Capel et al. 1972; Deichmann 1944; Edwards et al. 1986; French et al. 1974; Kao et al. 1979; Kenyon et al. 1995; Liao and Oehme 1981). In three human subjects who received a single oral dose of $0.01 \mathrm{mg} / \mathrm{kg}$ 
$\left[{ }^{14} \mathrm{C}\right]$-labeled phenol, the mean 24 -hour urinary recovery of ${ }^{14} \mathrm{C}$ was $90 \%$ (range $85-90 \%$ ) of the administered dose (Capel et al. 1972). In this same study, urinary recovery of orally administered $\left[{ }^{14} \mathrm{C}\right]$-labeled phenol was determined in 18 other mammalian species; the mean 24-hour recoveries of ${ }^{14} \mathrm{C}$ ranged from $95 \%$ in the rat to $31 \%$ in the squirrel monkey. In three separate fatal cases of ingestion of phenol at unknown quantities, phenol was detected in the urine at concentrations of $3.3 \mu \mathrm{g} / \mathrm{mL}$ (Boatto et al. 2004), $208 \mu \mathrm{g} / \mathrm{mL}$ (Tanaka et al. 1998), and $47 \mu \mathrm{g} / \mathrm{mL}$ (Lo Dico et al. 1989).

Both urinary and fecal excretion of ${ }^{14} \mathrm{C}$ was determined in rats administered an oral dose of $1.2 \mathrm{mg} / \mathrm{kg}$ of $\left[{ }^{14} \mathrm{C}\right]$-labeled phenol (Edwards et al. 1986). Rats excreted $80.3 \pm 11.2 \%$ in the urine and $1.8 \pm 1.6 \%$ in the feces in 24 hours. In rats exposed orally to radiolabeled phenol, elimination was $95 \%$ complete after 72 hours, with the primary elimination route being through the urine (Hughes and Hall 1995). Fecal elimination was slower and less overall.

Hiser et al. (1994) reported results of rats exposed to single and multiple bolus and drinking water doses of different concentrations of $\left[{ }^{14} \mathrm{C}\right]$-phenol. After 24 hours, concentrations of administered ${ }^{14} \mathrm{C}$ radioactivity were $>90 \%$ in the urine, regardless of the dose method or concentration. Less than $1 \%$ remained in tissues and carcass. This indicates rapid elimination of phenol in urine after oral exposure.

\subsubsection{Dermal Exposure}

Phenol absorbed through the skin is rapidly excreted in urine as free phenol or conjugates. A 4.5-hour occlusive exposure of one foot of an adult male to $90 \%$ phenol resulted in an elimination half-life of 13.86 hours. When admitted to the hospital, the phenol urine concentration was $7,909 \mathrm{mg} / \mathrm{g}$ in creatine. Over the next 12, 20,43, 58, and 82 hours, phenol concentrations in the urine were measured at 13,416, $721,80,62.8$, and $35.7 \mathrm{mg} / \mathrm{g}$ creatine, respectively (Bentur et al. 1998). Following an industrial accident in which a phenol-water solution was splashed over a man's face, chest wall, hand, and both arms, phenol in the urine decreased from $566 \mathrm{mg} / \mathrm{L}$ after 4 hours to $0.75 \mathrm{mg} / \mathrm{L} 46$ hours after the exposure (Horch et al. 1994). Subjects exposed to dermally applied reservoirs containing phenol solutions $(2.5-10 \mathrm{mg} / \mathrm{L})$ excreted $80 \%$ (range 58-98\%) of the absorbed phenol in the urine within 24 hours (BaranowskaDutkiewicz 1981). Another study in which both clothed and unclothed human subjects were dermally exposed for 7 hours to phenol vapors while breathing clean air to avoid inhalation exposure, found that almost $100 \%$ of the absorbed phenol was excreted in the urine within 1 day, with clothing providing no apparent protection (Piotrowski 1971). 
In rats exposed dermally to radiolabeled phenol, elimination was $95 \%$ complete after 72 hours, with the primary elimination route being through the urine (Hughes and Hall 1995). Fecal elimination was slower and less overall.

\subsubsection{Other Routes of Exposure}

Observations of elimination of phenol (5-10 mL 7\%) after injection into 20 patients were carried out as part lumbar of lumbar and thoracic sympathetic blockade treatments. Apparent elimination half-lives were $30.3 \pm 2.8$ minutes for unconjugated phenol and $64.0 \pm 7.3$ minutes for conjugated phenol. Urinary excretion of conjugated phenol was $52 \pm 5 \%$ after 8 hours (Nomoto et al. 1987).

\subsubsection{Physiologically Based Pharmacokinetic (PBPK)/Pharmacodynamic (PD) Models}

Physiologically based pharmacokinetic (PBPK) models use mathematical descriptions of the uptake and disposition of chemical substances to quantitatively describe the relationships among critical biological processes (Krishnan et al. 1994). PBPK models are also called biologically based tissue dosimetry models. PBPK models are increasingly used in risk assessments, primarily to predict the concentration of potentially toxic moieties of a chemical that will be delivered to any given target tissue following various combinations of route, dose level, and test species (Clewell and Andersen 1985). Physiologically based pharmacodynamic (PBPD) models use mathematical descriptions of the dose-response function to quantitatively describe the relationship between target tissue dose and toxic end points.

PBPK/PD models refine our understanding of complex quantitative dose behaviors by helping to delineate and characterize the relationships between: (1) the external/exposure concentration and target tissue dose of the toxic moiety, and (2) the target tissue dose and observed responses (Andersen and Krishnan 1994; Andersen et al. 1987). These models are biologically and mechanistically based and can be used to extrapolate the pharmacokinetic behavior of chemical substances from high to low dose, from route to route, between species, and between subpopulations within a species. The biological basis of PBPK models results in more meaningful extrapolations than those generated with the more conventional use of uncertainty factors.

The PBPK model for a chemical substance is developed in four interconnected steps: (1) model representation, (2) model parameterization, (3) model simulation, and (4) model validation (Krishnan and Andersen 1994). In the early 1990s, validated PBPK models were developed for a number of toxicologically important chemical substances, both volatile and nonvolatile (Krishnan and Andersen 
1994; Leung 1993). PBPK models for a particular substance require estimates of the chemical substancespecific physicochemical parameters, and species-specific physiological and biological parameters. The numerical estimates of these model parameters are incorporated within a set of differential and algebraic equations that describe the pharmacokinetic processes. Solving these differential and algebraic equations provides the predictions of tissue dose. Computers then provide process simulations based on these solutions.

The structure and mathematical expressions used in PBPK models significantly simplify the true complexities of biological systems. If the uptake and disposition of the chemical substance(s) are adequately described, however, this simplification is desirable because data are often unavailable for many biological processes. A simplified scheme reduces the magnitude of cumulative uncertainty. The adequacy of the model is, therefore, of great importance, and model validation is essential to the use of PBPK models in risk assessment.

PBPK models improve the pharmacokinetic extrapolations used in risk assessments that identify the maximal (i.e., the safe) levels for human exposure to chemical substances (Andersen and Krishnan 1994). PBPK models provide a scientifically sound means to predict the target tissue dose of chemicals in humans who are exposed to environmental levels (for example, levels that might occur at hazardous waste sites) based on the results of studies where doses were higher or were administered in different species. Figure 3-4 shows a conceptualized representation of a PBPK model.

If PBPK models for phenol exist, the overall results and individual models are discussed in this section in terms of their use in risk assessment, tissue dosimetry, and dose, route, and species extrapolations.

\subsubsection{Summary of PBPK Models}

A PBPK model simulating phenol disposition has been developed as part of the attempt to understand the toxicity of benzene, of which phenol is the primary metabolite (Bois et al. 1991). Human exposure to benzene is widespread, and much of the toxicity of benzene is due to the action of its metabolites. Thus, while no studies were located involving PBPK models developed specifically for phenol exposure, the benzene model of Bois et al. (1991) is capable of predicting the pharmacokinetics of phenol and is appropriate to this discussion. 


\section{Figure 3-4. Conceptual Representation of a Physiologically Based Pharmacokinetic (PBPK) Model for a Hypothetical Chemical Substance}

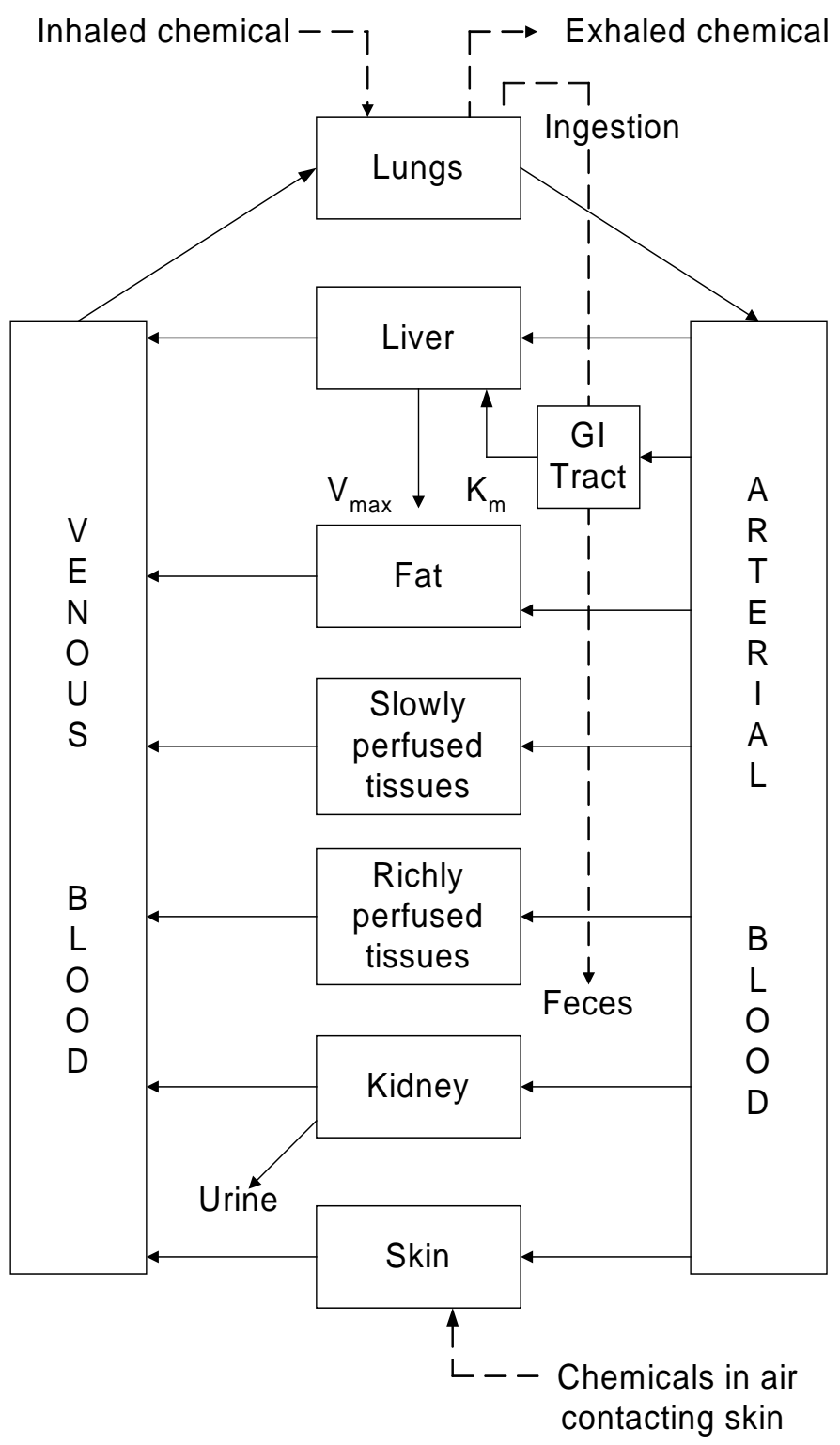

Source: adapted from Krishnan et al. 1994

Note: This is a conceptual representation of a physiologically based pharmacokinetic (PBPK) model for a hypothetical chemical substance. The chemical substance is shown to be absorbed via the skin, by inhalation, or by ingestion, metabolized in the liver, and excreted in the urine or by exhalation. 
Two empirical compartmental models for benzene have also been developed, which predict the production and subsequent metabolism of phenol. A two-compartment model for quantifying benzene hepatic metabolism to phenol and other metabolites in an in vitro microsome system was developed by Schlosser et al. (1993) and enhanced by Medinsky et al. (1995). This model does not predict benzene (or phenol) absorption, disposition, or excretion from the body of animals or humans. A one-compartment model of phenol in humans was developed for assessing the variability of worker biomarkers related to occupational exposures (Pierrehumbert et al. 2002). Methods for model calibration and validation data were not reported. Both of these models are limited for use in phenol risk assessment since they do not reduce uncertainties associated with extrapolation animal internal dosimetry to humans or high exposure levels to low levels.

\subsubsection{Discussion of Models}

\section{The Bois et al. (1991) Model}

A PBPK model for benzene and phenol was developed by Bois et al. (1991) to explore differences in metabolite formation and distribution from benzene or phenol exposures to clarify why benzene, but not phenol, is carcinogenic in rats and humans.

Description of the model. The model represents the male rat as a series of flow-limited compartments interconnected by arterial and alveolar blood flow. The disposition of benzene and phenol was predicted in the liver, fat, bone marrow, and well- and poorly-perfused tissues. In addition, phenol distribution to the gut and lung was included. Routes of exposure included oral gavage dosing, inhalation, and intravenous injection of both compounds. Elimination of benzene was accomplished by exhalation and metabolism in the liver and bone marrow. Additionally, phenol was conjugated in the liver, lung, and gut. Hepatic and bone marrow metabolism of benzene to phenol was described by Michaelis-Menten generation of benzene oxide followed by first-order production of phenol. MichaelisMenten kinetics described the transformation of benzene oxide to diols or glutathione conjugates and phenol to hydroquinone and sulfo- and glucurono-conjugates. Physiological flow and metabolic rates and constants were allometrically scaled to body weight. Instead of using point estimates of physiological and metabolic parameters values, uniform or log-uniform distributions (e.g., ranges) of parameter values were defined for all 64 model parameters. The model was executed using Monte Carlo techniques, in which individual random values were sampled from each parameter distribution during iterative model runs to produce distributions of model outputs rather than single values. This was done to accommodate 
variability and uncertainty in the parameter values. The parameter distributions were taken from the literature.

The model was calibrated to rat data for gavage (Sabourin et al. 1987, 1989) or inhalation (Sabourin et al. 1987, 1989) of benzene and intravenous, intra-arterial, intra-duodenal, and hepatic portal injection of phenol (Cassidy and Houston 1984) by adjusting the bounds of alveolar ventilation and other nonspecified parameter distributions. Different compartments pertaining to separate metabolic systems were assessed by selective injection as follows: jugular vein to assess first-pass metabolism across lung, hepatic portal vein to assess hepatic first-pass metabolism, duodenum to assess intestinal mucosa metabolism, and carotid artery to assess immediate tissues distribution.

Validation of the model. Validation of this model against empirical data was not done, introducing uncertainty into the ability of the model to predict other data. Of particular interest is the prediction that hydroquinone production is greater following phenol administration as compared to benzene administration. This is in opposition to the prediction of Medinsky et al. (1995).

Target tissues. The target tissues were blood and bone marrow. The expected levels of phenol in blood and bone marrow, and total hydroquinone were substantially higher after phenol administration than after benzene administration.

Species extrapolation. Extrapolation of this model from rats to other animals or humans has not been done.

Interroute extrapolation. The model included parameter values for intestinal absorption and pulmonary partitioning of phenol, which enable simulation of oral and inhalation exposures, respectively. However, calibration and validation of these routes was not performed against empirical data. Predictions of phenol metabolism and distribution following injection into various sites were used to illustrate possible consequences of first-pass metabolism following oral exposure to phenol.

Risk assessment. This model has not been applied to a quantitative risk assessment of benzene or phenol. The predicted blood and bone marrow phenol and total hydroquinone levels were substantially higher after phenol administration than after benzene administration. This finding is counter to the hypothesis that phenol or hydroquinone plays a direct role in the carcinogenicity of benzene, suggesting that other metabolites must be involved. The study authors suggest that catechol, a potentially genotoxic 
oxidation metabolite produced in much larger amounts following benzene, as opposed to phenol administration, may contribute to benzene's carcinogenicity.

\subsection{MECHANISMS OF ACTION}

\subsubsection{Pharmacokinetic Mechanisms}

Absorption of phenol occurs fairly rapidly via the inhalation (Hughes and Hall 1995; Ohtsuji and Ikeda 1972; Piotrowski 1971), oral (Capel et al. 1972; Edwards et al. 1986; French et al. 1974; Hughes and Hall 1995; Kao et al. 1979; Kenyon et al. 1995), and dermal (Baranowska-Dutkiewicz 1981; Hughes and Hall 1995; Piotrowski 1971) routes. Because it is an irritant, tissue damage, inflammation, or other irritation effects may occur at the sites of absorption. Because of its high $\mathrm{pK}_{\mathrm{a}}$, ionization will not occur within the acid environment of the gut. The action of gut microflora on phenol breakdown is not expected to be significant.

When it is absorbed through the lungs, gut, or skin, phenol conjugated at the portal-of-entry and free phenol enter the bloodstream where it can then be distributed throughout the body (if the conjugation capacity of the tissue has been saturated by a high dose). The dilution of phenol in water enhances the dermal absorption of phenol, as indicated by the greater toxicity of a water-phenol solution compared to neat phenol (Conning and Hayes 1970). Conning and Hayes (1970) speculated that an undiluted solution may produce a coagulative necrosis, which would slow further penetration of phenol resulting in less phenol absorbed than with more diluted solutions.

As described in Section 3.4.3, Metabolism, conjugation with glucuronic acid and conjugation with sulfate are the main routes of detoxification of phenol. In most species tested, including humans, sulfation predominates at lower doses. As doses increase, glucuronidation increases, as does the formation of oxidative metabolites. Some have suggested that the shift from sulfation to glucuronidation is caused by a reduction in the availability of co-substrates in conjugation reaction and/or reduction in the sulfate pool (Kim et al. 1995) or due to a difference in $K_{m}$ of the two pathways in relation to their respective $V_{\max }$ (Weitering et al. 1979). An alternative explanation for the dose-dependent metabolic profiles for phenol is that the activities of metabolizing enzymes vary across areas of the liver (Medinsky et al. 1995). As blood flows into the liver from the periphery of the lobule towards the central vein, it encounters first a zone in which both sulfotransferases and glucuronosyltransferases are present (periportal zone 1), the former predominating. Glucuronosyltransferases predominate in zone 2, whereas both glucuronosyltranferases and monooxygenases are present in pericentral zone 3. In this zonal arrangement, phenol 
would be metabolized first by sulfotransferases, and at low doses, little free phenol would be available for glucuronide conjugation and oxidation. However, at increasing phenol doses, unconjugated phenol that reaches zone 2 is available for glucuronidation. At even higher doses that exceed the conjugation capacities of zones 1 and 2, oxidative metabolites are generated (Kenyon et al. 1995). Studies in isolated perfused liver from rats (Ballinger et al. 1995) and mice (Hoffmann et al. 1999) have validated the 'enzyme zonation' model.

The influence of enzyme localization on intestinal metabolism of phenol has also been studied (Kothare and Zimmerman 2002). In an in situ perfused intestine preparation from rat, the investigators showed that sulfation was the predominant metabolic pathway after vascular administration of phenol, whereas luminal dosing produced greater glucuronidation. These results were consistent with the sulfotransferases being cytosolic enzymes (Burchell and Coughtrie 1997) and glucuronyltransferase being located between the nuclear and apical membrane of the epithelial cell (Inoue et al. 1999) and showing a decreasing expressional gradient from the villus to the crypt (Chowdhury et al. 1985).

Phenol that is absorbed is rapidly excreted in the urine as free phenol or conjugates (BaranowskaDutkiewicz 1981; Capel et al. 1972; Deichmann 1944; Edwards et al. 1986; French et al. 1974; Hughes and Hall 1995; Kao et al. 1979; Kenyon et al. 1995; Liao and Oehme 1981; Piotrowski 1971).

\subsubsection{Mechanisms of Toxicity}

Limited information is available regarding the mechanism(s) of toxicity of phenol. Phenol is irritating and corrosive at high concentrations as evidenced by numerous cases of accidental dermal exposure or intentional or accidental ingestion of phenol. Phenol impairs the stratum corneum and produces coagulation necrosis by denaturing and precipitating proteins. Studies in mice suggest that dermal application of phenol increases the formation of free radicals in the skin, and that the redox cycling of these radicals reduces antioxidant capacity, leading to significant oxidative damage of protein, DNA, and lipids (Murray et al. 2007).

Phenol is a hydroxylated metabolite of benzene and it further undergoes oxidative metabolism to produce other compounds; however, it is still unknown with certainty whether the parent compound or a metabolite(s) is responsible for phenol's systemic toxicity. The major tissues in which metabolism appears to occur are the liver, gut, lung, and kidney (Cassidy and Houston 1984; Powell et al. 1974; Quebbemann and Anders 1973; Tremaine et al. 1984). A study by Chapman et al. (1994) provided some 
insight on a possible toxic entity. These investigators found that incubation of whole rat conceptus in vitro with phenol resulted in minor dysmorphogenic and embryotoxic effect. However, addition of exogenous hepatic bioactivation system greatly increased the toxicity of phenol. The major metabolites formed were hydroquinone, catechol, and benzoquinone and these three metabolites exhibited similar potency. Chapman et al. (1994) also found that adding together phenol and hydroquinone resulted in more-than-additive embryotoxicity which, according to the investigators, suggested the involvement of a peroxidative mechanism for phenol bioactivation.

Several studies in animals have reported tremors following exposure by oral gavage (Moser et al. 1995; NTP 1983b). The mechanism by which phenol or metabolites exert this effect is unknown. There is little indication from studies in animals or from fatal poisoning cases in humans that phenol distributes preferentially to the brain, although tremors also may be caused by actions at the periphery. Injections of phenol (2-3\%) have been used to block nerve conduction in a number of neurological disorders (i.e., spasticity in cerebral palsy, cervical dystonia) or to relieve pain in certain cancers. This occurs by phenol physically interrupting the continuity of axons and inducing axonal degeneration. How this may be related to tremors caused by gavage dosing of phenol, if at all, is unknown. It has been suggested that phenol exposure results in cardiac effects because it blocks the cardiac sodium channel subtype, with little effect on sodium channels in skeletal muscle (Zamponi et al. 1994). A preferential block by phenol of sodium channels in inhibitory pathways would be consistent with a net result of increased activity or even tremors, but there is no experimental support for this hypothesis.

\subsubsection{Animal-to-Human Extrapolations}

Although mammals all metabolize phenol to the same metabolites, the amounts of each metabolite vary between species. For example, in the old world monkeys and prosimians, sulfation is the major phenol conjugation pathway, while in the new world monkeys, glucuronidation predominates (Mehta et al. 1978). Cats and pigs have low activities of phenol glucuronyltransferase, and metabolize phenol to phenyl sulfate nearly exclusively (Capel et al. 1972; French et al. 1974; Miller et al. 1976). Because humans have a greater capacity to glucuronidate phenol, cats and pigs would not be good models for the metabolism of phenol by humans.

\subsection{TOXICITIES MEDIATED THROUGH THE NEUROENDOCRINE AXIS}

Recently, attention has focused on the potential hazardous effects of certain chemicals on the endocrine system because of the ability of these chemicals to mimic or block endogenous hormones. Chemicals 
with this type of activity are most commonly referred to as endocrine disruptors. However, appropriate terminology to describe such effects remains controversial. The terminology endocrine disruptors, initially used by Thomas and Colborn (1992), was also used in 1996 when Congress mandated the EPA to develop a screening program for "...certain substances [which] may have an effect produced by a naturally occurring estrogen, or other such endocrine effect[s]...”. To meet this mandate, EPA convened a panel called the Endocrine Disruptors Screening and Testing Advisory Committee (EDSTAC), and in 1998, the EDSTAC completed its deliberations and made recommendations to EPA concerning endocrine disruptors. In 1999, the National Academy of Sciences released a report that referred to these same types of chemicals as hormonally active agents. The terminology endocrine modulators has also been used to convey the fact that effects caused by such chemicals may not necessarily be adverse. Many scientists agree that chemicals with the ability to disrupt or modulate the endocrine system are a potential threat to the health of humans, aquatic animals, and wildlife. However, others think that endocrine-active chemicals do not pose a significant health risk, particularly in view of the fact that hormone mimics exist in the natural environment. Examples of natural hormone mimics are the isoflavinoid phytoestrogens (Adlercreutz 1995; Livingston 1978; Mayr et al. 1992). These chemicals are derived from plants and are similar in structure and action to endogenous estrogen. Although the public health significance and descriptive terminology of substances capable of affecting the endocrine system remains controversial, scientists agree that these chemicals may affect the synthesis, secretion, transport, binding, action, or elimination of natural hormones in the body responsible for maintaining homeostasis, reproduction, development, and/or behavior (EPA 1997). Stated differently, such compounds may cause toxicities that are mediated through the neuroendocrine axis. As a result, these chemicals may play a role in altering, for example, metabolic, sexual, immune, and neurobehavioral function. Such chemicals are also thought to be involved in inducing breast, testicular, and prostate cancers, as well as endometriosis (Berger 1994; Giwercman et al. 1993; Hoel et al. 1992).

Based on the available information, there is no clear evidence that phenol is an endocrine disruptor in humans or in animals. Long-term studies in rats and mice treated with phenol in the drinking water did not report alterations in the gross or microscopic appearance of the reproductive organs (NCI 1980). In the 13-week experiment, rats and mice received approximately up to 1,700 and 2,700 $\mathrm{mg}$ phenol/kg/day, respectively. In the 2-year study, rats received estimated doses of phenol of up to $600-700 \mathrm{mg} / \mathrm{kg} / \mathrm{day}$ and mice received $1,100-1,200 \mathrm{mg} / \mathrm{kg} / \mathrm{day}$. Similar observations were made in a more recent two-generation reproductive study in rats (Ryan et al. 2001). In the latter study, the highest doses of phenol, 301-321 mg/kg/day, had no significant effect on fertility, estrus frequency, testicular sperm count, or sperm motility or morphology. Significant reductions in prostate and uterine weights in all $\mathrm{F}_{1}$ 
treated groups were not considered adverse effects of phenol by Ryan et al. (2001) on the basis of the absence of histological alterations and functional reproductive effects, and based on the fact that only a few animals had organ weights outside the range of concurrent control values.

In standard developmental toxicity studies in rats and mice, with one exception, fetotoxicity has only been reported at doses that were also toxic to the mothers (Narotsky and Kavlock 1995; NTP 1983b; Ryan et al. 2001; York 1997). In the study by NTP (1983a) in rats, a 7\% decrease in fetal body weight was reported at the high-dose level, $120 \mathrm{mg} / \mathrm{kg} /$ day, without any evidence of maternal toxicity. However, historical control data showed that the concurrent control fetal weight for the CD rat was much higher $(22 \%)$ than the historical control weight. In addition, a larger litter size in the high-dose group may have contributed to the smaller fetal weight in the high-dose group.

Only two reports were located with relevant information from assays in vitro. In one of them, phenol tested negative for estrogenic activity in a reporter gene expression assay using yeast cells (Nishihara et al. 2000). A substance was considered positive when its activity was $>10 \%$ of the activity of $10^{-7} \mathrm{M} 17 \beta$ estradiol. For phenol, that concentration was $>1 \times 10^{-3} \mathrm{M}$. The other study found that phenol had very weak binding affinity to a purified recombinant human estrogen receptor (Hu and Aizawa 2003).

\subsection{CHILDREN'S SUSCEPTIBILITY}

This section discusses potential health effects from exposures during the period from conception to maturity at 18 years of age in humans, when all biological systems will have fully developed. Potential effects on offspring resulting from exposures of parental germ cells are considered, as well as any indirect effects on the fetus and neonate resulting from maternal exposure during gestation and lactation. Relevant animal and in vitro models are also discussed.

Children are not small adults. They differ from adults in their exposures and may differ in their susceptibility to hazardous chemicals. Children's unique physiology and behavior can influence the extent of their exposure. Exposures of children are discussed in Section 6.6, Exposures of Children.

Children sometimes differ from adults in their susceptibility to hazardous chemicals, but whether there is a difference depends on the chemical (Guzelian et al. 1992; NRC 1993). Children may be more or less susceptible than adults to health effects, and the relationship may change with developmental age (Guzelian et al. 1992; NRC 1993). Vulnerability often depends on developmental stage. There are 
critical periods of structural and functional development during both prenatal and postnatal life, and a particular structure or function will be most sensitive to disruption during its critical period(s). Damage may not be evident until a later stage of development. There are often differences in pharmacokinetics and metabolism between children and adults. For example, absorption may be different in neonates because of the immaturity of their gastrointestinal tract and their larger skin surface area in proportion to body weight (Morselli et al. 1980; NRC 1993); the gastrointestinal absorption of lead is greatest in infants and young children (Ziegler et al. 1978). Distribution of xenobiotics may be different; for example, infants have a larger proportion of their bodies as extracellular water, and their brains and livers are proportionately larger (Altman and Dittmer 1974; Fomon 1966; Fomon et al. 1982; Owen and Brozek 1966; Widdowson and Dickerson 1964). The infant also has an immature blood-brain barrier (Adinolfi 1985; Johanson 1980) and probably an immature blood-testis barrier (Setchell and Waites 1975). Many xenobiotic metabolizing enzymes have distinctive developmental patterns. At various stages of growth and development, levels of particular enzymes may be higher or lower than those of adults, and sometimes unique enzymes may exist at particular developmental stages (Komori et al. 1990; Leeder and Kearns 1997; NRC 1993; Vieira et al. 1996). Whether differences in xenobiotic metabolism make the child more or less susceptible also depends on whether the relevant enzymes are involved in activation of the parent compound to its toxic form or in detoxification. There may also be differences in excretion, particularly in newborns who all have a low glomerular filtration rate and have not developed efficient tubular secretion and resorption capacities (Altman and Dittmer 1974; NRC 1993; West et al. 1948). Children and adults may differ in their capacity to repair damage from chemical insults. Children also have a longer remaining lifetime in which to express damage from chemicals; this potential is particularly relevant to cancer.

Certain characteristics of the developing human may increase exposure or susceptibility, whereas others may decrease susceptibility to the same chemical. For example, although infants breathe more air per kilogram of body weight than adults breathe, this difference might be somewhat counterbalanced by their alveoli being less developed, which results in a disproportionately smaller surface area for alveolar absorption (NRC 1993).

Based on a very limited data set, it is likely that most of the effects of phenol exposure, including cardiac arrhythmias and central nervous system depression, observed in adults after exposure to high amounts of phenol will be observed in children if exposures are comparable. The data are insufficient to determine whether children will be especially sensitive to such effects, however. 
IARC (1989), citing Hinkel and Kintzel (1968), indicated that a newborn infant whose umbilicus had been bound with a bandage containing $2 \%$ phenol, died after 11 hours. Another newborn whose skin ulcer was treated with a solution of $30 \%$ phenol $/ 60 \%$ camphor developed circulatory failure, cerebral intoxication, and methemoglobinemia, but recovered after a blood transfusion. Rogers et al. (1978) evaluated the percutaneous absorption of phenol in 16 infants, aged 2-5 months, who were treated for seborrhoeic eczema with Magenta Paint B.P.C., a medicine containing 4\% phenol. The treatment consisted of twice daily painting of the napkin and skin folds (representing about 11-15\% of the body surface) with the Magenta paint over 48 hours, with an average of $32 \mathrm{~mL}$ of paint (approximately 1,300 $\mathrm{mg}$ of phenol) applied to each child. Phenol was detected in the urine of four of the infants; however, no information on concentration was presented. Liver function tests run on 8 of the 16 treated infants showed no abnormalities. The study was initiated because of the observation of signs of central nervous system depression in a 6-month-old who had been treated over a much larger area (all of the body except the face).

A study of 2,075 infants exposed to phenolic disinfectants used to clean hospital nursery surfaces reported a significant increase in mean third-day microbilirubin level and an increase in the proportion of infants with a microbilirubin level $>10 \mathrm{mg} / \mathrm{dL}$ (Doan et al. 1979). However, no cases of severe jaundice were observed. Since infants did not come into direct contact with the cleaned surfaces, exposure was assumed to have occurred by inhalation of fumes.

In a 5-year (1987-1991) retrospective review of acute exposures to a phenol-containing disinfectant (Creolin Disinfectant ${ }^{\mathrm{TM}}$ [26\% phenol]) reported to a regional poison control center, Spiller et al. (1993) identified 96 patients, 16 of which were lost to follow-up. There were 60 oral-only exposures, 7 dermalonly exposures, 12 oral/dermal exposures, and 1 inhalation exposure. Sixty (75\%) of the patients were under 5 years of age. It was not possible to determine from the information presented the degree of concordance between the 60 patients with oral-only exposures and the 60 under the age of 5, but it is clear that oral exposure of young children is the predominant characteristic of this population of exposed individuals. In this regard, children have clearly been demonstrated to be at greater risk of exposure to phenol via the accidental ingestion of phenol-containing disinfectants. Vomiting and lethargy were the main signs of toxicity observed in the children.

Warner and Harper (1985) reported the case of a 10-year-old male developed cardiac arrhythmias following a chemical peeling procedure initiated to remove a $12 \times 17 \mathrm{~cm}$ hairy nevus of the left scapula and nape. An hour into the procedure, which involved the application of a solution of phenol (60\% 
phenol, $0.8 \%$ croton oil in hexachlorophene soap and water) to the entire surface of the nevus, multifocal and coupled premature ventricular complexes developed in the electrocardiogram, but subsided after infusion of bretylium sulfate. Although this was a severe reaction, it is difficult to determine, based on just one case, whether it reflects a special sensitivity based on age. A 10-year-old boy who was hospitalized with serious burns was treated dermally with $7.5 \mathrm{~L}$ of an antiseptic solution containing $2 \%$ phenol for 2.5 days. During this period, his urine became dark, his respiration became labored, he fell into a coma, and he died. Postmortem analysis of urine revealed $200 \mathrm{mg} / \mathrm{L}$ of conjugated phenol (Cronin and Brauer 1949).

In a review of the use of phenol as a neurolytic agent, Wood (1978) summarized the results of a number of studies including one in which children with cerebral palsy were given nerve blocks with $3 \%$ phenol in water as a treatment for spasticity. Out of 150 blocks on 46 children, 9 were associated with complications, 8 with muscle weakness, and 1 with painful paresthesia. This degree of complication was twice that reported by another group who reported on 98 blocks, presumably in adults, with a complication rate of $3 \%$ with all complications being transient paresthesia. The first group concluded that in children the risk was too great for the benefit of the procedure. These two studies in combination suggest that children may be especially sensitive to phenol given by injection. Interestingly, a later study (Morrison et al. 1991) involving 24 pediatric patients similarly treated for spasticity with injections of 5\% phenol in water at the motor point of insertion during halothane anesthesia concluded that there was no increase in the incidence of complications. In this study the complications of concern were cardiac arrhythmias and the incidence was $19 \%$, yet the authors concluded that the procedure appeared "appropriate to perform in the day-surgery context." The difference in these studies is likely due to the fact that the earlier study evaluated the incidence of delayed complications, whereas the Morrison et al. (1991) work evaluated the incidence of an immediate complication, e.g., cardiac arrhythmias. There was no indication in the Morrison et al. (1991) study that delayed complications such as subsequent muscle weakness or paresthesia were evaluated.

Only one study in animals was located that compared the age-dependency toxicity of phenol. Deichmann and Witherup (1944) administered phenol orally and subcutaneously to three age groups of rats: 10 days old, 5 weeks old, and adults. At a dose of $600 \mathrm{mg} / \mathrm{kg}$ orally, death occurred in $90 \%$ of 10 -day-old rats, in $30 \%$ of 5 -week-old rats, and in $60 \%$ of adult rats. Similarly, 3,000 mg/kg administered subcutaneously caused death in $65 \%$ of 10 -day-old rats, $25 \%$ of 5 -week-old rats, and $45 \%$ of adult animals. These results suggested that neonates are more sensitive than adults, and that adults are more sensitive than young rats, but these findings have not been confirmed. 
Extremely limited data regarding possible adverse developmental effects in humans exposed to phenol provide no evidence for effects. As mentioned in Section 3.6, standard developmental toxicity studies in rats and mice, with one exception (NTP 1983a), have reported fetotoxicity at doses that were also toxic to the mothers (Narotsky and Kavlock 1995; NTP 1983b; Ryan et al. 2001; York 1997). Results from some studies in vivo and in vitro suggest that phenol potentially could affect the germ cells, opening the possibility that parental exposure would result in adverse childhood development or cancer (Bulsiewicz 1977; Li et al. 2005). However, the results of a well-conducted two-generation reproduction study do not support that possibility (Ryan et al. 2001).

There is no information regarding pharmacokinetics of phenol in children. As discussed in Section 3.4.3, phenol is metabolized by CYP2E1 isozymes and also forms sulfate and glucuronide conjugates. To the extent that the enzymes involved in the metabolism of phenol are developmentally regulated, the metabolism, and consequently the toxicity of phenol, in immature humans may be different than in adults. If microsomal oxidation transforms phenol into a toxic metabolite, a reduced CYP2E1 activity, as it seems to occur in neonates, would result in decreased toxicity. However, the ability of the liver to sulfonate phenol, and consequently facilitate elimination, also develops with age (Iwasaki et al. 1993). Thus, a lower ability to conjugate could result in more phenol available for oxidative metabolism. Heaton and Renwick (1991) found that young rats have a higher production of oxidative metabolism than adult rats. If this were the case in humans, children might be potentially more sensitive to the systemic effects of phenol. Glucuronide conjugation reactions also are considerably reduced in the young and reach adult values only after the age of 3 in humans. This would play a role at high doses where the glucuronide metabolites of phenol predominate. As previously mentioned, caution should be exercised when extrapolating from adolescent rats to children, since rodents are known to undergo a number of changes in xenobiotic-metabolizing enzymes during sexual development (Waxman et al. 1985).

It is not known whether phenol can cross the placenta and there are no reports on levels of phenol in maternal milk.

There are no biomarkers of exposure or effects for phenol that have been validated in children or in adults exposed as children. No relevant studies were located regarding interactions of phenol with other chemicals in children or adults. 


\subsection{BIOMARKERS OF EXPOSURE AND EFFECT}

Biomarkers are broadly defined as indicators signaling events in biologic systems or samples. They have been classified as markers of exposure, markers of effect, and markers of susceptibility (NAS/NRC 1989).

Due to a nascent understanding of the use and interpretation of biomarkers, implementation of biomarkers as tools of exposure in the general population is very limited. A biomarker of exposure is a xenobiotic substance or its metabolite(s) or the product of an interaction between a xenobiotic agent and some target molecule(s) or cell(s) that is measured within a compartment of an organism (NAS/NRC 1989). The preferred biomarkers of exposure are generally the substance itself, substance-specific metabolites in readily obtainable body fluid(s), or excreta. However, several factors can confound the use and interpretation of biomarkers of exposure. The body burden of a substance may be the result of exposures from more than one source. The substance being measured may be a metabolite of another xenobiotic substance (e.g., high urinary levels of phenol can result from exposure to several different aromatic compounds). Depending on the properties of the substance (e.g., biologic half-life) and environmental conditions (e.g., duration and route of exposure), the substance and all of its metabolites may have left the body by the time samples can be taken. It may be difficult to identify individuals exposed to hazardous substances that are commonly found in body tissues and fluids (e.g., essential mineral nutrients such as copper, zinc, and selenium). Biomarkers of exposure to phenol are discussed in Section 3.8.1.

Biomarkers of effect are defined as any measurable biochemical, physiologic, or other alteration within an organism that, depending on magnitude, can be recognized as an established or potential health impairment or disease (NAS/NRC 1989). This definition encompasses biochemical or cellular signals of tissue dysfunction (e.g., increased liver enzyme activity or pathologic changes in female genital epithelial cells), as well as physiologic signs of dysfunction such as increased blood pressure or decreased lung capacity. Note that these markers are not often substance specific. They also may not be directly adverse, but can indicate potential health impairment (e.g., DNA adducts). Biomarkers of effects caused by phenol are discussed in Section 3.8.2.

A biomarker of susceptibility is an indicator of an inherent or acquired limitation of an organism's ability to respond to the challenge of exposure to a specific xenobiotic substance. It can be an intrinsic genetic or other characteristic or a preexisting disease that results in an increase in absorbed dose, a decrease in the 
biologically effective dose, or a target tissue response. If biomarkers of susceptibility exist, they are discussed in Section 3.10, Populations That Are Unusually Susceptible.

\subsubsection{Biomarkers Used to Identify or Quantify Exposure to Phenol}

Biological monitoring for exposure to phenol is possible by measuring blood or urine levels of the parent compound. However, it should be noted that phenol and metabolites of phenol may also come from other sources. For example, phenol is a metabolite of benzene and of protein metabolism. Urine samples taken from male workers employed in the distillation of high-temperature phenolic fractions of tar revealed a phenol excretion rate of $4.20 \mathrm{mg} / \mathrm{hour}$ compared to a control rate of $0.53 \mathrm{mg} /$ hour for non-exposed workers (Bieniek 1994). Samples were taken 4 hours into the workers' workday, but the worker exposure levels were not reported. A study of workers in a Bakelite factory reported a linear correlation between concentrations of phenol in the air (up to $12.5 \mathrm{mg} / \mathrm{m}^{3}$ or $3.25 \mathrm{ppm}$ ) and urinary excretion of total phenol (free plus conjugated) (Ohtsuji and Ikeda 1972). However, the urinary concentration of free phenol seemed to be independent of the environmental phenol, suggesting that under the exposure conditions, the maximum capacity to conjugate phenol had not been reached.

The biological exposure index (BEI) for occupational exposure to $5 \mathrm{ppm}$ phenol is $250 \mathrm{mg}$ total phenol in urine/g creatinine (ACGIH 2005). The urine should be collected at the end of the 8-hour work shift. The sample can be stored in the refrigerator for 4 days or frozen for at least 3 months before analysis. ACGIH (2005) warns that the test is nonspecific and should not be used when workers are exposed to benzene or to household products or medications that contain phenol. Dermal exposure may result in overestimation of inhalation exposure.

Phenol can also be measured in the urine after oral exposure, although a dose-response relationship between oral exposure to phenol and phenol in the urine has not been established. In persons not exposed to phenol or benzene, the total phenol concentration in the urine does not exceed $20 \mathrm{mg} / \mathrm{L}$ and is usually $<10 \mathrm{mg} / \mathrm{L}$ (ACGIH 2005).

\subsubsection{Biomarkers Used to Characterize Effects Caused by Phenol}

Specific biomarkers used to characterize effects caused by phenol have not been identified. Dark urine has been reported in persons exposed to phenol (orally, dermally, or by inhalation) (Baker et al. 1978; Bentur et al. 1998; Cronin and Brauer 1949; Kim et al. 1994; Merliss 1972). The dark urine may be a result of an oxidation product of phenol or hemoglobin or hemoglobin breakdown products. Further 
research is required to identify the cause of the dark urine. If it is the result of an oxidation product of phenol, it should be considered a biomarker of exposure.

For more information on biomarkers for renal and hepatic effects of chemicals see ATSDR/CDC Subcommittee Report on Biological Indicators of Organ Damage (Agency for Toxic Substances and Disease Registry 1990) and for information on biomarkers for neurological effects see OTA (1990).

\subsection{INTERACTIONS WITH OTHER CHEMICALS}

Phenol is a tumor promoter in laboratory animals. In mice, dermal exposure to phenol in benzene (Boutwell and Bosch 1959) or in acetone (Salaman and Glendenning 1957; Wynder and Hoffmann 1961) increased the incidence of tumors resulting from dermal exposure to the tumor initiator, 9,10-DMBA. The mechanism of phenol promotion activity is not known, but may be related to the dermal damage that it causes and subsequent rapid cell division that may take place to repair the damage. When injected with mixtures of phenol and hydroquinone, a hydroxylated metabolite of phenol, mice exhibited significantly depressed bone marrow erythropoiesis compared to injection with phenol alone (Chen and Eastmond 1995a). The involvement of peripheral acetylcholine in phenol-induced tremors was implicated by studies in which mice were injected with phenol and pentobarbital, an inhibitor of acetylcholine release (Itoh 1995).

\subsection{POPULATIONS THAT ARE UNUSUALLY SUSCEPTIBLE}

A susceptible population will exhibit a different or enhanced response to phenol than will most persons exposed to the same level of phenol in the environment. Reasons may include genetic makeup, age, health and nutritional status, and exposure to other toxic substances (e.g., cigarette smoke). These parameters result in reduced detoxification or excretion of phenol, or compromised function of organs affected by phenol. Populations who are at greater risk due to their unusually high exposure to phenol are discussed in Section 6.7, Populations with Potentially High Exposures.

Potentially, individuals with low activities of the enzymes phenol sulfotransferase and glucuronyltransferase may be more susceptible to phenol toxicity. Persons with ulcerative colitis may have an impaired capacity to sulfate phenol (Ramakrishna et al. 1991), which may increase the amount of unchanged phenol that is absorbed following oral exposure. Neonates may also be more susceptible to toxicity from dermally-applied phenol because of increased skin permeability and proportionately greater surface area. A study in which 10-day-old rats were more sensitive to lethality following oral exposure to 
phenol than 5-week-old or adult rats (Deichmann and Witherup 1944) further suggests that the young may be more sensitive to phenol. For a more detailed discussion, please see Section 3.7. Because phenol is a vesicant, individuals with sensitive skin or pulmonary incapacity may be more sensitive to phenol. Individuals with kidney or liver diseases that impair metabolism or excretion of phenol and phenol metabolites may be more susceptible to phenol.

\subsection{METHODS FOR REDUCING TOXIC EFFECTS}

This section will describe clinical practice and research concerning methods for reducing toxic effects of exposure to phenol. However, because some of the treatments discussed may be experimental and unproven, this section should not be used as a guide for treatment of exposures to phenol. When specific exposures have occurred, poison control centers and medical toxicologists should be consulted for medical advice. The following texts provide specific information about treatment following exposures to phenol:

Goldfrank LR, Flomenbaum NE, Lewin NA, et al. 2002. Goldfrank's toxicologic emergencies. 7th ed. New York, NY: McGraw-Hill.

Haddad LM, Shannon MW, Winchester JF. 1998. Clinical management of poisoning and drug overdose. 3rd ed. Philadelphia, PA: WB Saunders Company.

Viccellio P. 1998. Emergency toxicology. 2nd ed. Philadelphia, PA: Lippincott-Raven Publishers.

\subsubsection{Reducing Peak Absorption Following Exposure}

Human exposure to phenol may occur by inhalation, ingestion, or dermal contact. Mitigation methods for reducing exposure to phenol include the general recommendation of separating contaminated food, water, air, and clothing from the exposed individual. Externally, phenol can produce mild irritation; acute exposure may produce dermatitis and abnormal pigmentation (HSDB 2008). Dermal exposure to relatively low concentrations of phenol (5-6\%) over a sufficient surface area can result in death. Therefore, speed in removing phenol from the skin is important (HSDB 2008). A study was conducted to assess the efficacy of 10 different strategies to decontaminate the skin of anesthetized pigs that were exposed to $89 \%$ aqueous phenol for 1 minute (Monteiro-Riviere et al. 2001). The treatment included plain water washes for different periods of time, as well as soap, polyethylene glycol, and isopropanol solutions. The evaluation was based on scoring of erythema, edema, and histological parameters of the skin. The result showed polyethylene glycol (PEG 400) and 70\% isopropanol were superior to the other treatments and equally effective in reducing skin damage. The study also found that PEG 400, 70\% 
isopropanol, and 15-minute water treatments significantly reduced phenol absorption in the isolated porcine skin flap relative to no treatment.

Emesis is not recommended following oral ingestion of phenol because of phenol's corrosive effects and potential for seizures and rapid central nervous system depression. Instead, in the absence of esophageal injury, repeated gastric lavage is recommended followed by administration of olive oil or vegetable oil to remove surface phenol and prevent deeper penetration. This can be followed by administration of a cathartic such as castor oil, sorbitol, or saline. Lavage is contraindicated if esophageal injury is suspected.

\subsubsection{Reducing Body Burden}

Phenol is excreted in the breath, urine, and feces. Mitigation strategies to increase urinary output and dilute the chemical once it is in the bloodstream may be useful. One method for this may be increased hydration of the individual in order to stimulate diuresis. Hemodialysis is not effective in removing phenol. Information on the distribution of phenol is limited and provides little insight on how distribution might be altered to facilitate any attempts at mitigation of effects.

\subsubsection{Interfering with the Mechanism of Action for Toxic Effects}

The mechanism of action of phenol in the body is not well understood. Reports of cardiac arrhythmias resulting from phenol exposure are not uncommon (Gross 1984; Horch et al. 1994; Truppman and Ellenby 1979; Warner and Harper 1985). Specific methods to interfere with the mechanism of action for phenol were not identified. Treatment of phenol toxicity is essentially supportive. Patients exposed by inhalation should be removed from the contaminated area and given 100\% humidified oxygen and ventilatory assistance. Cardiovascular support includes the use of intravenous saline and vasopressors to support the blood pressure. Lidocaine can be used to treat ventricular dysrhythmias and bretilium for lidocaine-refractory arrhythmias. Administration of sodium bicarbonate intravenously may rapidly reverse central nervous system depression in the presence of metabolic acidosis. Also, if methemoglobinemia is $>30 \%$, ingestion of methylene blue may be warranted.

\subsection{ADEQUACY OF THE DATABASE}

Section 104(I)(5) of CERCLA, as amended, directs the Administrator of ATSDR (in consultation with the Administrator of EPA and agencies and programs of the Public Health Service) to assess whether adequate information on the health effects of phenol is available. Where adequate information is not 
available, ATSDR, in conjunction with the National Toxicology Program (NTP), is required to assure the initiation of a program of research designed to determine the health effects (and techniques for developing methods to determine such health effects) of phenol.

The following categories of possible data needs have been identified by a joint team of scientists from ATSDR, NTP, and EPA. They are defined as substance-specific informational needs that if met would reduce the uncertainties of human health assessment. This definition should not be interpreted to mean that all data needs discussed in this section must be filled. In the future, the identified data needs will be evaluated and prioritized, and a substance-specific research agenda will be proposed.

\subsubsection{Existing Information on Health Effects of Phenol}

The existing data on health effects of inhalation, oral, and dermal exposure of humans and animals to phenol are summarized in Figure 3-5. The purpose of this figure is to illustrate the existing information concerning the health effects of phenol. Each dot in the figure indicates that one or more studies provide information associated with that particular effect. The dot does not necessarily imply anything about the quality of the study or studies, nor should missing information in this figure be interpreted as a "data need". A data need, as defined in ATSDR's Decision Guide for Identifying Substance-Specific Data Needs Related to Toxicological Profiles (Agency for Toxic Substances and Disease Registry 1989), is substance-specific information necessary to conduct comprehensive public health assessments. Generally, ATSDR defines a data gap more broadly as any substance-specific information missing from the scientific literature.

The existing information on the health effects of phenol in humans comes from case reports of people who accidentally or intentionally swallowed phenol-containing substances or whose skin came in contact with phenol, subjects exposed to phenol (and possibly to other substances at the same time) at work, and populations whose drinking water was contaminated with phenol (and other substances). Acute oral and dermal exposure to high amounts of phenol caused serious systemic effects and even death in humans due phenol's irritant and corrosive properties.

Acute- and intermediate-duration inhalation studies in animals are available, but only one of these studies can be considered a modern study. Inhalation studies in animals showed adverse respiratory, cardiovascular, liver, renal, and neurological effects. Phenol administered by oral gavage is much more toxic than in drinking water; this is related to differences in toxicokinetics between the two means of 
Figure 3-5. Existing Information on Health Effects of Phenol

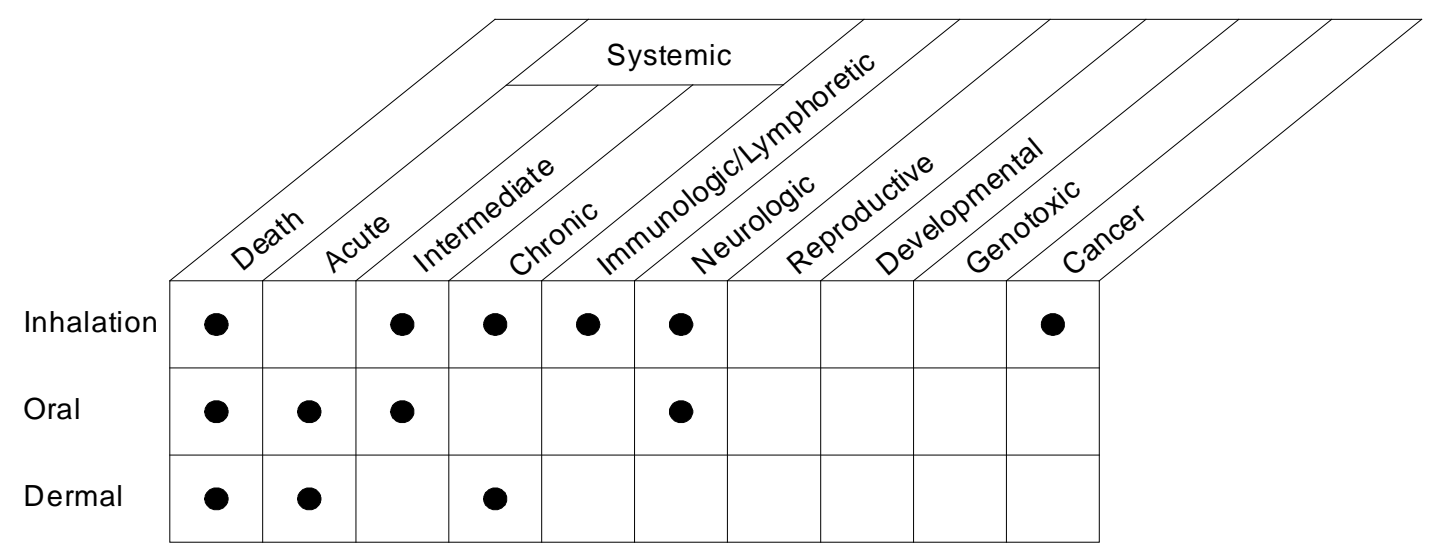

Human

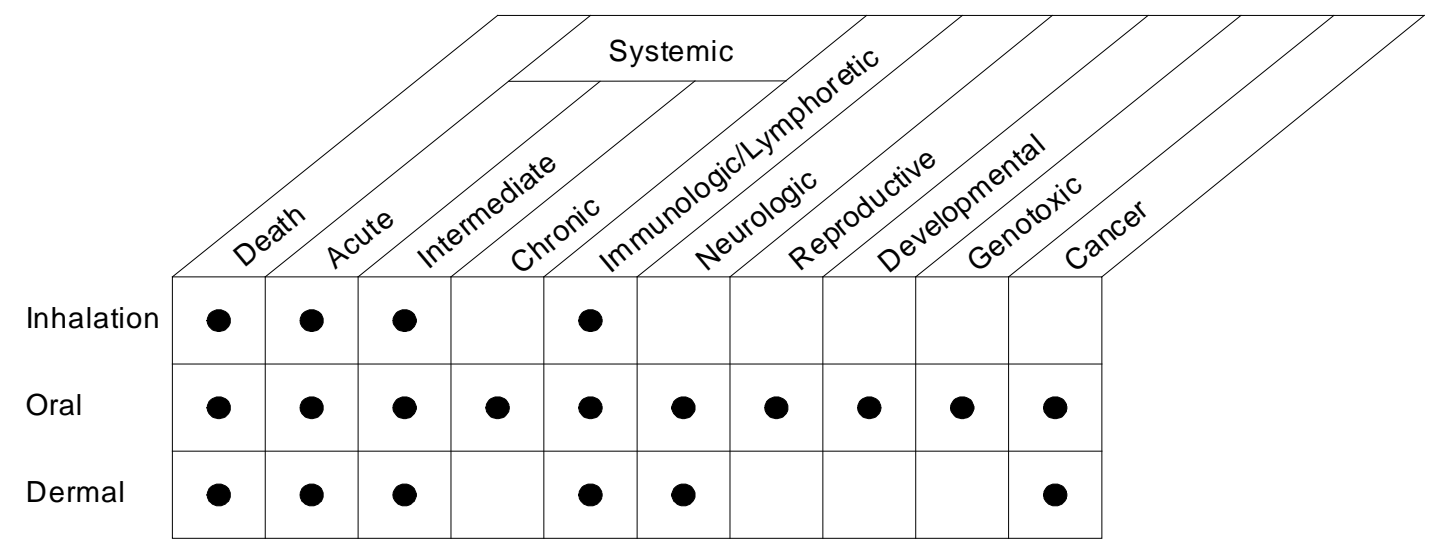

Animal

Existing Studies 
administration. Phenol exhibited relatively little toxicity in drinking water studies, including studies of reproductive and developmental end points. Of special interest is a study that reported hematological, immunological, and neurological effects in mice exposed to low concentrations of phenol in the drinking water for 28 days. Longer-term studies examined mostly systemic end points. Data are extensive regarding genotoxicity of phenol in bacterial systems and mammalian systems. Data regarding the oral carcinogenicity of phenol in rats and mice are available, as well as data on the dermal carcinogenicity, and tumor-promoting and tumor-inhibiting activities of phenol.

\subsubsection{Identification of Data Needs}

Acute-Duration Exposure. Case reports of humans exposed to high doses of phenol, either orally or dermally, have provided acute toxicity information. Both ingestion of phenol (Boatto et al. 2004; Soares and Tift 1982; Stajduhar-Caric 1968; Tanaka et al. 1998) and contact of phenol with a significant area of the skin (Cronin and Brauer 1949; Griffiths 1973; Soares and Tift 1982) have caused deaths in humans. The cardiovascular system also might be considered a target for acute phenol toxicity. Supraventricular and ventricular dysrhythmias were reported in a case of acute ingestion of phenol (Langford et al. 1998) and cardiac arrhythmia and bradycardia were reported following acute dermal exposure to phenol (Gross 1984; Horch et al. 1994; Truppman and Ellenby 1979; Warner and Harper 1985). Two acute-duration inhalation studies in animals of limited scope indicated that the respiratory tract and the nervous system are targets for phenol toxicity (De Ceaurriz et al. 1981; Flickinger 1976). A more recent well-conducted study that used modern methodology to evaluate a number of relevant end points, including upper and lower respiratory tract histology, defined a study NOAEL of $25 \mathrm{ppm}$, the highest exposure level tested (Hoffman et al. 2001). Because no adverse effects were reported in that study, it was considered an unsuitable basis for an MRL. Therefore, additional studies may be necessary to establish NOAELs and LOAELs that can be used to construct dose-response relationships for acute exposure to phenol.

Acute-duration oral gavage studies in animals provided information on lethal doses (Berman et al. 1995; Deichmann and Witherup 1944; Flickinger 1976; von Oettingen and Sharpless 1946) and other effects, including renal (Berman et al. 1995), hematological (Ciranni et al. 1988), neurological (Moser et al. 1995), and developmental effects (Narotsky and Kavlock 1995; NTP 1983a, 1983b). A study in which pregnant rats were administered phenol by oral gavage, but divided in three daily doses and in a relatively high volume to minimize the effects of a bolus dose of phenol, reported a significant reduction in body weight gain in the dams at $\geq 120 \mathrm{mg} / \mathrm{kg} /$ day, but no significant developmental effects were reported at this dose level (York 1997). The maternal NOAEL was $60 \mathrm{mg} / \mathrm{kg} /$ day. Decreased fetal weight and decreased 
ossification sites were only seen at the highest dose level, $360 \mathrm{mg} / \mathrm{kg} /$ day. The reduction in maternal weight gain during pregnancy was used as the basis for the derivation of an acute-duration oral MRL of $1 \mathrm{mg} / \mathrm{kg} /$ day for phenol. A need for additional acute-duration oral studies is not apparent at this time. Phenol is a well known skin irritant and further acute-duration dermal studies in animals are unlikely to provide new key information.

Intermediate-Duration Exposure. Limited information exists regarding effects of phenol in humans exposed for intermediate durations. A study of office workers exposed to vapors of a liquid wood preservative containing phenol, among a number of other chlorinated compounds, reported adverse respiratory, hematological, hepatic, and ocular effects, but the specific role of phenol, if any, could not be ascertained (Baj et al. 1994). Studies of populations whose drinking water was contaminated with phenol found increased incidences of nausea and diarrhea, but exposure to chlorophenols may have also occurred (Baker et al. 1978; Jarvis et al. 1985; Kim et al. 1994). Only three studies were identified that exposed animals to airborne phenol for intermediate durations (Dalin and Kristoffersson 1974; Deichmann et al. 1944; U.S. Air Force 1961). These studies provided evidence of respiratory, heart, liver, kidney, and neurological effects, but had numerous limitations including poor control of exposure levels, unclear scope of the evaluations, and limited reporting, and were inadequate for MRL derivation. Therefore, a well-conducted 90-day inhalation study that examines a comprehensive number of end points would provide valuable information for dose-response analyses and possibly MRL derivation. Since phenol is well absorbed through the skin, a nose-only exposure protocol may be considered.

Several studies provided information on the effects of phenol following intermediate-duration oral exposure and all of them used drinking water to administer phenol. A 13-week drinking water study in rats and mice evaluated clinical signs and gross and microscopic appearance of a number of organs and tissues and found little evidence of toxicity (NCI 1980). A two-generation reproduction study found no evidence of reproductive effects in male or female rats, but reported decreased pup weight and reduced viability (Ryan et al. 2001). A specialized 13-week neurotoxicity study reported decreased motor activity in female rats (Beyrouty 1998). In these three studies, the doses tested were higher than doses tested in acute-duration oral studies. The most significant findings in the intermediate-duration oral database were those of Hsieh et al. (1992) who reported hematological, neurochemical, and immunological effects in mice at dose levels much lower than those used in other studies that tested similar end points. The Hsieh et al. (1992) study was not used for derivation of an intermediate-duration oral MRL largely due to the unconfirmed nature of findings and because only five mice comprised each dose group. Replication of Hsieh's findings seems necessary before using the data for risk assessment. Skin ulcerations were 
reported in mice treated dermally with 20\% phenol in acetone once each week for 24-32 weeks (Salaman and Glendenning 1957). Because humans are more likely to be dermally exposed to phenol in water, and phenol is readily absorbed through the skin, additional intermediate-duration studies examining the effects of dermal exposure to different concentrations of phenol in water may fill a data gap.

Chronic-Duration Exposure and Cancer. There is limited information on health effects in humans exposed chronically to phenol. Neither morbidity nor mortality was significantly increased in workers in five companies that used formaldehyde and phenol (Dosemeci et al. 1991). In another study of workers in the rubber industry, exposure to phenol was associated with an increased incidence of cardiovascular disease, independently of being associated with exposure to other solvents such as carbon disulfide (Wilcosky and Tyroler 1983). Liver effects, as judged by increased serum activities of ALT and AST, were reported in a case of prolonged inhalation exposure to phenol (Merliss 1972) and in workers in an oil-refining plant (Shamy et al. 1994), but exposure to other solvents could not be ruled out in the latter case. The lack of exposure data and simultaneous exposure to other chemicals precluded using the human data for derivation of a chronic-duration inhalation MRL for phenol. No chronic-duration inhalation studies in animals were identified. This constitutes a data gap that may need to be filled.

The only chronic-duration animal studies are the NCI (1980) 103-week studies in rats and mice. NCI (1980) evaluated clinical signs, organ weights, and gross and microscopic appearance of organs and tissues. Under the conditions of the study, phenol showed essentially no systemic toxicity. A chronicduration oral drinking water study with emphasis on immunological end points may be necessary if the intermediate-duration immunotoxicity study that NTP is currently conducting confirms that immunocompetence is the most sensitive end point for phenol toxicity. Since, as previously mentioned, phenol is readily absorbed through the skin and the possibility exists of dermal exposure via contaminated water (bathing or showering) at or near waste sites, a chronic-duration dermal study of phenol in water may be considered if the results of a shorter-term study suggest that adverse effects might happen.

A study of phenol-exposed wood industry workers reported a small, nonsignificant excess of respiratory cancers (Kauppinen et al. 1986) and a study of phenol production workers reported a small, nonsignificant excess of Hodgkin's disease and of lung, esophageal, and kidney cancers (Dosemeci et al. 1991). However, the interpretation of these findings is complicated due to lack of dose-response and potential for confounding. Phenol has been tested for carcinogenicity in long-term drinking water bioassays in rats and mice (NCI 1980). Statistically significant increased incidences of pheochromocytomas of the adrenal gland and leukemia or lymphomas were observed in male rats 
exposed to the low dose of phenol, but not to the high dose of phenol. No significant effects were seen in female rats or in mice. Additional bioassays do not seem necessary at this time.

Genotoxicity. Phenol has been tested extensively for genotoxicity in a variety of in vivo (Amlacher and Rudolph 1981; Barale et al. 1990; Bulsiewicz 1977; Chen and Eastmond 1995a; Ciranni et al. 1988; Gocke et al. 1981; Li et al. 2005; Miyagawa et al. 1995; Pashin et al. 1987; Shelby and Witt 1995; Skare and Schrotel 1984; Sturtevant 1952) and in vitro (Crebelli et al. 1987; Demerec et al. 1951; Erexson et al. 1985; Florin et al. 1980; Gocke et al. 1981; Haworth et al. 1983; Jansson et al. 1986; Kubo et al. 2002; Li et al. 2005; Miller et al. 1995; Morimoto and Wolff 1980; Morimoto et al. 1983; Nagel et al. 1982; Painter and Howard 1982; Paschin and Bahitova 1982; Pellack-Walker and Blumer 1986; Poirier et al. 1975; Pool and Lin 1982; Schwartz et al. 1985; Sze et al. 1996; Tsutsui et al. 1997) tests. The results of these assays have been equivocal. Phenol appears to be potentially genotoxic, although this may be more a result of the action of its metabolites than the parent compound. Additional genotoxicity studies of phenol do not seem to be necessary.

Reproductive Toxicity. Extremely limited data regarding possible adverse reproductive effects in humans following exposure to phenol provide no evidence for effects (Axelsson et al. 1984). Histopathological changes in reproductive organs were not observed in rats or mice treated with phenol in the drinking water for 13 or 103 weeks (NCI 1980). In a two-generation study in which rats were administered phenol in the drinking water, there were no significant alterations in gross or microscopic appearance of the reproductive organs of males and females from the parental and $\mathrm{F}_{1}$ generations (Ryan et al. 2001). In addition, there were no significant effects on estrus frequency, testicular sperm count, or sperm motility or morphology. Significant reductions in prostate and uterine weights in all $F_{1}$ treated groups were not considered adverse effects of phenol by Ryan et al. (2001) on the basis of the absence of histological alterations and functional reproductive effects, and based on the fact that only a few animals had organ weights outside the range of concurrent control values. Additional reproductive toxicity studies by the oral route do not seem necessary. Studies by the inhalation or dermal route also do not seem necessary since there is no indication that reproductive effects would be route-dependent.

Developmental Toxicity. Extremely limited data regarding possible adverse developmental effects in humans following exposure to phenol provide no evidence for effects (Axelsson et al. 1984). Phenol has been evaluated for developmental effects in rats and mice exposed by oral gavage (Ciranni et al. 1988; Kavlock 1990; Narotsky and Kavlock 1995; NTP 1983a, 1983b; York 1997) and in rats dosed through the drinking water in a two-generation reproduction study (Ryan et al. 2001). These studies 
indicated that fetotoxicity occurs only at dose levels that are also toxic to the mother. The study by York (1997) was used to derive an acute-duration oral MRL of $1.0 \mathrm{mg} / \mathrm{kg} /$ day for phenol. Additional developmental studies by the oral route of exposure do not seem necessary at this time. Also, studies by the inhalation or dermal route also do not seem necessary since there is no indication that developmental effects would be route-dependent.

Immunotoxicity. Immunological effects were reported in workers exposed for 6 months to a mixture of phenol, formaldehyde, and organic chlorohydrocarbons, although there is some question whether the exposure was due to phenol or a substituted phenol (Baj et al. 1994). Increased susceptibility to bacteria was not observed in mice exposed by inhalation to phenol (Aranyi et al. 1986). Necrosis or atrophy of the spleen or thymus, which was not described further, was observed in rats given a single dose of phenol by oral gavage (Berman et al. 1995). Effects on the spleen or thymus were not observed in rats given 14 daily doses of phenol (Berman et al. 1995). Decreased antibody production in response to immunization with SRBC was observed in mice treated with relatively low doses of phenol in the drinking water for 28 days (Hsieh et al. 1992). Ryan et al. (2001) conducted similar tests in rats in the two-generation reproductive study and found no significant effects of phenol. It would be useful to try to replicate Hsieh's findings before the results can be used for risk assessment. In longer-term studies, histopathologic changes in the spleen or thymus were not observed in rats or mice exposed to phenol in the drinking water for 13 or 103 weeks, but immunocompetence was not assessed (NCI 1980). Studies by the inhalation and dermal routes are not necessary since there is no evidence of route-dependency.

Neurotoxicity. An increase in the number of headaches was reported by persons exposed to phenol in drinking water following an accident, but chlorophenols may have contributed to the observed effects (Kim et al. 1994). As reported in a retrospective review (Spiller et al. 1993), 11 patients with oral exposures to phenol-based disinfectants experienced rapid central nervous system depression, but no seizures occurred. Neurological effects (muscle tremor, loss of coordination) have been reported in laboratory animals after single exposures to high concentrations of phenol in the air (Flickinger 1976), continuous exposure in the air (Dalin and Kristoffersson 1974), repeated intermittent exposures in the air (Deichmann et al. 1944), and oral gavage dosing (Deichmann and Witherup 1944; Liao and Oehme 1981; Moser et al. 1995; NTP 1983b). In contrast, no such effects were observed in rats and mice in drinking water studies of longer durations and with higher doses of phenol (Beyrouty 1998; NCI 1980). These neurological effects correlate with peak blood concentrations of phenol achieved during gavage dosing. Drinking water studies suggest that the nervous system is not a sensitive target for phenol toxicity by this 
route of exposure. A need to conduct additional toxicity studies is not apparent, but studies aimed at elucidating the mechanism(s) of phenol neurotoxicity are needed.

Epidemiological and Human Dosimetry Studies. As previously mentioned, information about the health effects of phenol in humans is derived from studies of workers and members of the general population following inhalation, oral, and dermal exposure. Specific effects and references are mentioned in previous sections. Doses were generally not available, but Deichmann and Keplinger (1981) estimated that an oral dose as low as $1 \mathrm{~g}$ could be fatal in humans, but also pointed out that patients occasionally survived doses as high as $65 \mathrm{~g}$. Other than the skin and mucosal membranes, the liver and cardiovascular system might by considered targets for phenol toxicity. Wilcosky and Tyroler 1983 studied workers in the rubber industry and found that exposure to phenol was associated with an increased incidence of cardiovascular disease, independently of being associated with exposure to other solvents such as carbon disulfide. Cardiac arrhythmia and bradycardia were reported following acute dermal exposure to phenol (Gross 1984; Horch et al. 1994; Truppman and Ellenby 1979; Warner and Harper 1985) and supraventricular and ventricular dysrhythmias were reported in a case of acute ingestion of phenol (Langford et al. 1998). Liver effects were reported in a case of prolonged inhalation exposure to phenol (Merliss 1972) and in workers in an oil-refining plant (Shamy et al. 1994), but exposure to other solvents could not be ruled out in the latter case. Prolonged exposure to low levels of phenol may occur at or near waste sites via contaminated water. Since such contamination may lead to inhalation exposure (evaporation of phenol when bathing or showering, particularly if the water is acidic), dermal exposure (absorption through the skin), and oral exposure (ingestion of water or cooking with contaminated water), a dosimetric model that predicts total exposure and intake of phenol from contaminated water would be valuable. The specific end points that should be monitored under such exposure scenario (prolonged low level) are not immediately apparent. Phenol administered in the drinking water to rats and mice for 2 years showed almost no systemic toxicity (NCI 1980).

\section{Biomarkers of Exposure and Effect.}

Exposure. Measurement of total phenol in the urine is the most useful biomarker following inhalation exposure to phenol (ACGIH 2001). The test is nonspecific and should not be used when workers are exposed to benzene, to household products, or to medications containing phenol. Dermal exposure may also result in overestimation of inhalation exposure. In persons not exposed to phenol or benzene, the total phenol concentration in the urine does not exceed $20 \mathrm{mg} / \mathrm{L}$ and the mean is usually $<10 \mathrm{mg} / \mathrm{L}$ (ACGIH 2001). Phenol can also be measured in the urine after oral exposure, although a dose-response 
relationship between oral exposure to phenol and phenol in the urine has not been established. Benzene metabolism yields not only phenol, 1,4-dihydroxybenzene, and their sulfates and glucuronides, but also the benzene-specific $t, t$-muconic acid. For both $t, t$-muconic acid and $S$-phenylmercapturic acid, significant correlations were shown with benzene concentrations in air and in blood (Popp et al. 1994; Stommel et al. 1989). Thus, determination of urinary concentrations of these metabolites allows delineation of the portion of metabolites stemming from phenols and the portion derived from benzene exposure. Further research on the relationship between exposure doses and urinary levels of phenol is needed.

Effect. Specific biomarkers used to characterize effects caused by phenol have not been identified. Dark urine has been reported in persons occupationally exposed to phenol (inhalation, dermal) (ACGIH 2001; Merliss 1972), and following oral exposure (Baker et al. 1978; Kim et al. 1994). The dark urine may be a result of an oxidation product of phenol or hemoglobin. Further research is required to identify the cause of the dark urine.

Absorption, Distribution, Metabolism, and Excretion. The toxicokinetics of phenol have been studied extensively in laboratory animals and humans. Phenol is readily absorbed from the lungs, gastrointestinal tract, and skin. A study that examined the absorption of phenol vapor through the skin indicates that it is readily absorbed and clothing does not serve as a barrier (Piotrowski 1971). Dermal absorption is considered the primary route of entry for vapor, liquid, and solid phenol (ACGIH 2001). Conjugation of phenol with glucuronic acid and sulfate are the main detoxification pathways. Conjugation occurs predominantly in the lungs, gastrointestinal tract, liver, and kidneys. The skin has relatively low potential to detoxify phenol. Therefore, absorption through the skin may represent the greatest hazard from phenol because it readily passes through the skin and because there is no first-pass metabolic effect as is observed following oral exposure. Further studies regarding the metabolism of phenol following dermal exposure are needed. In vitro studies of phenol metabolism have demonstrated that reactive intermediates are produced during the metabolism of phenol (Chapman et al. 1994; Eastmond et al. 1986; Lunte and Kissinger 1983; Subrahmanyam and O'Brien 1985). These reactive compounds may be involved in mediating phenol toxicity. Further investigation of these compounds in tissues suspected of being targets for phenol toxicity (i.e., the lungs, skin, liver, kidney, and heart) are needed to provide information for extrapolating from animals to humans.

There is no PBPK model specifically designed for phenol, although phenol, as a major metabolite of benzene, has been considered in a PBPK model of benzene discussed in this profile (Bois et al. 1991). 
The model does not adequately explain the differences in carcinogenicity observed between benzene and phenol, and needs additional refinements in order to incorporate all the observations and be validated. Additional efforts to develop a PBPK model for phenol are needed.

Comparative Toxicokinetics. The metabolism and excretion of orally administered phenol in 18 animal species have been compared to metabolism and excretion in humans (Capel et al. 1972). The rat was the most similar to the human with respect to the fraction of administered dose excreted in urine in 24 hours (95\%) and the number and relative abundance of the four principal metabolites excreted in urine (sulfate and glucuronide conjugates of phenol and 1,4-dihydroxybenzene). The rat excreted a larger fraction of the orally administered dose than the guinea pig or the rabbit (Capel et al. 1972) and appears to be the least susceptible of the three species to respiratory, cardiovascular, hepatic, renal, and neurological effects of inhaled phenol (Deichmann et al. 1944). More rapid metabolism and excretion of absorbed phenol may account for the lower sensitivity of the rat to systemic effects of phenol. More information on the relative rates of metabolism of phenol in various species is needed to identify the most appropriate animal model for studying potential health effects in humans.

Methods for Reducing Toxic Effects. Removing a person from phenol exposure is the most important method for reducing toxic effects of phenol. This is especially important following dermal exposure, after which speed in removing phenol from the skin is important (HSDB 2008). Because a study has shown that dilution in water increases the dermal absorption of phenol (Conning and Hayes 1970), it has been recommended that polyethylene be used to remove dermal contamination with phenol (Viccellio 1998). Because water is readily available, others believe that its use is more appropriate for the decontamination of skin following phenol exposure (Pullin et al. 1978). A study is available that evaluated several strategies to decontaminate the skin of pigs following acute dermal exposure to phenol (Monteiro-Riviere et al. 2001). The study showed that polyethylene glycol (PEG 400) and 70\% isopropanol were superior to other treatments and equal effective in reducing skin damage induced by phenol. The general recommendations for reducing the absorption of phenol following acute oral exposure are well established and have a proven efficiency (HSDB 2008). No additional investigations are considered necessary at this time.

No clinical treatments, other than supportive measures, are currently available to enhance elimination of phenol following exposure. Studies designed to assess the potential risks or benefits of increasing ventilation to enhance pulmonary elimination or of stimulating excretion of phenol and its metabolic products are needed. 
Children's Susceptibility. Data needs relating to both prenatal and childhood exposures, and developmental effects expressed either prenatally or during childhood, are discussed in detail in the Developmental Toxicity subsection above.

Deichmann and Witherup (1944) found that 10-day-old rats were more sensitive to lethality following oral exposure to phenol than 5-week-old or adult rats; however, this work has never been repeated and there was little other information evaluating the toxicity of phenol at various ages. Such studies need to be conducted in order to follow up this earlier observation. However, caution should be exercised when extrapolating from adolescent rats to children, since rodents are known to undergo a number of changes in xenobiotic-metabolizing enzymes during sexual development (Waxman et al. 1985).

Studies that specifically evaluated age-related changes in the phase I and phase II metabolic transformations of phenol are not available. However, in general, it is known that there is a reduced capacity to metabolize xenobiotics in the first 15 days of life, and that the different enzyme systems have different time courses of development thereafter (Morselli et al. 1980). For example, glucuronide conjugation reactions are considerably reduced in young humans, and reach adult values only after the age of 3 , whereas sulfate conjugations and oxidative reactions catalyzed by the cytochrome P450 enzymes apparently develop more rapidly (Buxton 2006; Morselli et al. 1980). Thus, there could be age-related differences in the balance among metabolites, particularly at high doses where the glucuronide metabolites begin to dominate. While there is agreement that conjugation reactions represent a detoxification mechanism for phenol, it is still unknown whether parent compound or an oxidation product(s) is the entity responsible for the systemic toxicity of phenol in vivo. Studies are needed to examine how age affects the metabolism of phenol, and particularly how age changes the balance between phase I and phase II metabolism at either high or low doses.

There was no information found on the placental transfer of phenol or on the concentrations of phenol present in breast milk. There is evidence that benzene and its (not specifically identified) metabolites do cross the placenta, although there is no evidence of selective accumulation (Ghantous and Danielsson 1986). Additional studies of this issue are needed to determine if phenol and its metabolites are among the metabolites of benzene that cross the placenta, and if so whether phenol behaves like benzene in the lack of accumulation. Information is also needed on the content of phenol in breast milk under various conditions (e.g, smoking versus nonsmoking mothers) in order to determine if breast milk could ever be a source of phenol exposure for children. 
Child health data needs relating to exposure are discussed in Section 6.8.1, Identification of Data Needs: Exposures of Children.

\subsubsection{Ongoing Studies}

No ongoing studies pertaining to phenol were identified in the Federal Research in Progress database (FEDRIP 2006). 


\section{CHEMICAL AND PHYSICAL INFORMATION}

\subsection{CHEMICAL IDENTITY}

Information regarding the chemical identity of phenol is located in Table 4-1.

\subsection{PHYSICAL AND CHEMICAL PROPERTIES}

Information regarding the physical and chemical properties of phenol is located in Table 4-2. 
Table 4-1. Chemical Identity of Phenol

\begin{tabular}{|c|c|c|}
\hline Characteristic & Information & Reference \\
\hline Chemical name & Phenol & Lide 2005 \\
\hline Synonym(s) & $\begin{array}{l}\text { Benzenol, hydroxylbenzene, } \\
\text { monophenol, oxybenzene, phenyl } \\
\text { alcohol, phenyl hydrate, phenyl } \\
\text { hydroxide, phenylic acid, phenylic } \\
\text { alcohol }\end{array}$ & Lewis 2000 \\
\hline Registered trade name(s) & $\begin{array}{l}\text { Carbolic acid, phenic acid, phenic } \\
\text { alcohol }\end{array}$ & Gardner et al. 1978 \\
\hline Chemical formula & $\mathrm{C}_{6} \mathrm{H}_{6} \mathrm{O}$ & Lide 2005 \\
\hline Chemical structure & $\mathrm{OH}$ & Budavari et al. 1989 \\
\hline \multicolumn{3}{|l|}{ Identification numbers: } \\
\hline CAS registry & $108-95-2$ & HSDB 2008 \\
\hline NIOSH RTECS & SJ3325000 & RTECS 2006 \\
\hline EPA hazardous waste & U188 & EPA 1998; HSDB 2008 \\
\hline \multirow[t]{4}{*}{ DOT/UN/NA/IMCO shipping } & UN 1671 (solid) & HSDB 2008 \\
\hline & UN 2312 (molten) & \\
\hline & UN 2821 (solution) & \\
\hline & IMO 6.1 (solid, molten, solution) & \\
\hline HSDB & 113 & HSDB 2008 \\
\hline $\mathrm{NCl}$ & C50124 & Lewis 2000 \\
\hline
\end{tabular}

CAS = Chemical Abstracts Services; DOT/UN/NA/IMCO = Department of Transportation/United Nations/North America/International Maritime Dangerous Goods Code; EPA = Environmental Protection Agency; HSDB = Hazardous Substances Data Bank; NCI = National Cancer Institute; NIOSH = National Institute for Occupational Safety and Health; RTECS=Registry of Toxic Effects of Chemical Substances 
Table 4-2. Physical and Chemical Properties of Phenol

\begin{tabular}{|c|c|c|}
\hline Property & Information & Reference \\
\hline Molecular weight & 94.111 & Lide 2005 \\
\hline Color & Colorless to light pink & HSDB 2008 \\
\hline Physical state & Crystalline solid liquid $\left(w / 8 \% \mathrm{H}_{2} \mathrm{O}\right)$ & \\
\hline Melting point & $40.89^{\circ} \mathrm{C}$ & Lide 2005 \\
\hline Boiling point & $181.87^{\circ} \mathrm{C}$ & Lide 2005 \\
\hline Density at $20^{\circ} \mathrm{C} / 4^{\circ} \mathrm{C}$ & 1.0545 at $45^{\circ} \mathrm{C} / 4^{\circ} \mathrm{C}$ & Lide 2005 \\
\hline Vapor density & 3.24 & Lewis 2000 \\
\hline Odor & $\begin{array}{l}\text { Distinct aromatic, somewhat sickening, sweet } \\
\text { and acrid odor }\end{array}$ & HSDB 2008 \\
\hline \multicolumn{3}{|l|}{ Odor threshold: } \\
\hline \multirow[t]{2}{*}{ Water } & 7.9 ppm (w/v) & Amoore and Hautala 1983 \\
\hline & $1 \mathrm{ppm}(\mathrm{w} / \mathrm{v})$ & Baker et al. 1978 \\
\hline Air & $0.040 \mathrm{ppm}(\mathrm{v} / \mathrm{v})$ & Amoore and Hautala 1983 \\
\hline \multicolumn{3}{|l|}{ Solubility: } \\
\hline Water at $25^{\circ} \mathrm{C}$ & $8.28 \times 10^{4} \mathrm{mg} / \mathrm{L}$ & Southworth and Keller 1986 \\
\hline Organic solvent(s) & $\begin{array}{l}\text { Soluble in water and ethanol, very soluble in } \\
\text { ether, miscible with acetone and benzene }\end{array}$ & Lide 2005 \\
\hline \multicolumn{3}{|l|}{ Partition coefficients: } \\
\hline $\log \mathrm{K}_{\mathrm{ow}}$ & 1.46 & HSDB 2008 \\
\hline $\log \mathrm{K}_{\mathrm{oc}}$ & $1.21-1.96$ & $\begin{array}{l}\text { Artiola-Fortuny and Fuller } \\
\text { 1982; Boyd 1982; Briggs } \\
\text { 1981; Sacan and Balcioglu } \\
\text { 1996; Scott et al. } 1983\end{array}$ \\
\hline Vapor pressure at $25^{\circ} \mathrm{C}$ & $0.35 \mathrm{mmHg}$ & HSDB 2008 \\
\hline Henry's law constant & $3.0 \times 10^{7} \mathrm{atms} \mathrm{m}^{3}-\mathrm{mol}$ & Gaffney et al. 1987 \\
\hline Autoignition temperature & $715^{\circ} \mathrm{C}$ & Lewis 2000 \\
\hline Flashpoint, open cup & $85^{\circ} \mathrm{C}$ & HSDB 2008 \\
\hline Flashpoint, closed cup & $79^{\circ} \mathrm{C}$ & HSDB 2008 \\
\hline $\begin{array}{l}\text { Flammability limits (in air, } \\
\text { by } \% \text { v) }\end{array}$ & $1.7-8.6 \%$ & HSDB 2008 \\
\hline \multicolumn{3}{|l|}{ Conversion factors: } \\
\hline $\begin{array}{l}\text { ppm }(\mathrm{v} / \mathrm{v}) \text { to } \mathrm{mg} / \mathrm{m}^{3} \text { in } \\
\text { air }\left(25^{\circ} \mathrm{C}\right)\end{array}$ & $\mathrm{ppm}(\mathrm{v} / \mathrm{v}) \times 3.92=\mathrm{mg} / \mathrm{m}^{3}$ & \\
\hline $\begin{array}{l}\mathrm{mg} / \mathrm{m}^{3} \text { to } \mathrm{ppm}(\mathrm{v} / \mathrm{v}) \text { in } \\
\text { air }\left(25^{\circ} \mathrm{C}\right)\end{array}$ & $\mathrm{mg} / \mathrm{m}^{3} \times 0.225=\mathrm{ppm}(\mathrm{v} / \mathrm{v})$ & \\
\hline
\end{tabular}


4. CHEMICAL AND PHYSICAL INFORMATION

This page is intentionally blank. 


\section{PRODUCTION, IMPORT/EXPORT, USE, AND DISPOSAL}

\subsection{PRODUCTION}

Table 5-1 lists the number of facilities in each state that manufacture or process phenol, the intended use, and the range of maximum amounts of phenol that are stored onsite. The data listed in Table 5-1 are derived from the Toxics Release Inventory (TRI) (TRI05 2007). Only certain types of facilities were required to report. Therefore, this is not an exhaustive list.

Phenol has been obtained by distillation from petroleum and synthesis by oxidation of cumene or toluene, and by vapor-phase hydrolysis of chlorobenzene (Wallace 1998). In 2004, nearly 98\% of U.S. phenol production was based on oxidation of cumene except at one company that used toluene oxidation and a few companies that distilled phenol from petroleum (CMR 2005). In 2004, the total annual capacity of phenol production approached 6.6 billion pounds (CMR 2005). A list of current U.S. producers of phenol is found in Table $5-2$.

\subsection{IMPORT/EXPORT}

According to the National Trade Data Bank (USITC 2008), exports of phenol were 503 million $\mathrm{kg}$ (1,110 million pounds) (USITC 2008). The major importer of phenol from the United States was Canada, with an import value of 117 million $\mathrm{kg}$ during 2007. The total amount of phenol imported to the United States was 1.3 million $\mathrm{kg}$ ( 2.8 million pounds) in 2005. The largest exporter of phenol to the United States was South Africa, which exported 1 million kg of phenol (USITC 2008).

\subsection{USE}

The two major uses of phenol in 2004 were the production of bisphenol-A (48\%) and the production of phenolic resins (25\%) (CMR 2005). The largest use for bisphenol-A is as an intermediate in the production of epoxy resins (Thurman 1982). Phenol-formaldehyde resins comprise over $95 \%$ of this market (Thurman 1982). Other major uses of phenol include the production of caprolactam (11\%), aniline (2\%), alkylphenols (4\%), xylenols (4\%), and miscellaneous uses (6\%) (CMR 2005). Phenol is used as a slimicide (a chemical toxic to bacteria and fungi characteristic of aqueous slimes) and as a general disinfectant in solution or mixed with slaked lime for toilets, stables, cesspools, floors, drains, and other areas (Budavari et al. 1989; Hawley 1981). 


\section{Table 5-1. Facilities that Produce, Process, or Use Phenol}

\begin{tabular}{|c|c|c|c|c|}
\hline State $^{a}$ & $\begin{array}{l}\text { Number of } \\
\text { facilities }\end{array}$ & $\begin{array}{l}\text { Minimum } \\
\text { amount on site } \\
\text { in pounds }\end{array}$ & $\begin{array}{l}\text { Maximum } \\
\text { amount on site } \\
\text { in pounds }\end{array}$ & 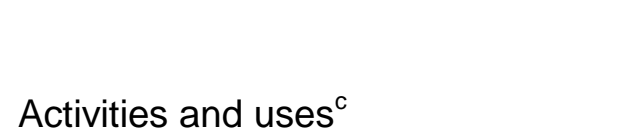 \\
\hline AK & 1 & 100 & 999 & 1,13 \\
\hline$A L$ & 93 & 0 & $49,999,999$ & $1,2,3,4,5,6,7,8,9,10,11,12,13,14$ \\
\hline AR & 39 & 0 & $49,999,999$ & $1,2,3,5,6,7,8,9,10,11,12,13,14$ \\
\hline$A Z$ & 17 & 0 & $9,999,999$ & $1,5,6,7,8,11,12$ \\
\hline $\mathrm{CA}$ & 122 & 0 & $9,999,999$ & $1,2,3,4,5,6,7,8,9,10,11,12,13,14$ \\
\hline $\mathrm{CO}$ & 6 & 0 & 99,999 & $1,2,5,6,9,10,11$ \\
\hline $\mathrm{CT}$ & 24 & 0 & 999,999 & $2,3,6,7,8,10,11,12,14$ \\
\hline DE & 10 & 1,000 & 999,999 & $1,3,5,6,7,12,13$ \\
\hline FL & 27 & 0 & $9,999,999$ & $1,5,6,7,8,11,12,13$ \\
\hline GA & 68 & 0 & $49,999,999$ & $1,2,3,4,5,6,7,8,10,11,12,13,14$ \\
\hline GU & 2 & 0 & 9,999 & 9 \\
\hline IA & 31 & 0 & 999,999 & $2,3,5,6,7,8,10,11,12$ \\
\hline ID & 6 & 0 & 9,999 & $1,2,3,5,7,11,13$ \\
\hline IL & 105 & 0 & $49,999,999$ & $1,2,3,4,5,6,7,8,9,10,11,12,13,14$ \\
\hline IN & 126 & 0 & $49,999,999$ & $1,2,3,4,5,6,7,8,9,10,11,12,13,14$ \\
\hline KS & 36 & 0 & $9,999,999$ & $1,2,3,4,5,6,7,8,10,11,12,13$ \\
\hline KY & 51 & 0 & $9,999,999$ & $1,2,3,4,5,6,7,8,9,10,11,12,13$ \\
\hline LA & 100 & 0 & $99,999,999$ & $1,2,3,4,5,6,7,8,9,10,11,12,13,14$ \\
\hline MA & 38 & 0 & $499,999,999$ & $1,2,3,5,6,7,8,10,11,12,13$ \\
\hline MD & 19 & 0 & 999,999 & $1,2,3,4,5,6,7,8,11,13$ \\
\hline ME & 23 & 0 & 999,999 & $1,2,3,5,6,7,8,10,12,13,14$ \\
\hline MI & 88 & 0 & $9,999,999$ & $1,2,3,4,5,6,7,8,9,10,11,12,13,14$ \\
\hline MN & 41 & 0 & $9,999,999$ & $1,2,3,4,5,6,7,8,9,10,11,12,13$ \\
\hline MO & 42 & 0 & $9,999,999$ & $1,2,3,4,5,6,7,8,9,10,11,12$ \\
\hline MS & 49 & 0 & $49,999,999$ & $1,2,3,4,5,6,7,8,9,10,11,12,13,14$ \\
\hline MT & 15 & 0 & $9,999,999$ & $1,2,3,5,6,9,13,14$ \\
\hline NC & 69 & 0 & $49,999,999$ & $1,2,3,4,5,6,7,8,9,10,11,12,13,14$ \\
\hline ND & 3 & 0 & 99,999 & $1,5,7$ \\
\hline NE & 9 & 100 & 999,999 & $1,3,5,6,8,11,12$ \\
\hline $\mathrm{NH}$ & 11 & 0 & 999,999 & $1,2,3,5,7,8,10,11,12,13$ \\
\hline NJ & 47 & 0 & $49,999,999$ & $1,2,3,4,5,6,7,8,9,10,11,12,13,14$ \\
\hline NM & 3 & 10,000 & 999,999 & $1,2,3,7,10,12,13$ \\
\hline NV & 2 & 1,000 & 99,999 & 7 \\
\hline NY & 62 & 0 & $9,999,999$ & $1,2,3,4,5,6,7,8,9,10,11,12,13,14$ \\
\hline $\mathrm{OH}$ & 122 & 0 & $499,999,999$ & $1,2,3,4,5,6,7,8,9,10,11,12,13,14$ \\
\hline OK & 45 & 0 & $9,999,999$ & $1,2,3,4,5,6,7,8,9,10,11,12,13,14$ \\
\hline OR & 50 & 0 & $49,999,999$ & $1,2,3,4,5,6,7,8,9,10,11,12,13$ \\
\hline PA & 108 & 0 & $99,999,999$ & $1,2,3,4,5,6,7,8,9,10,11,12,13$ \\
\hline
\end{tabular}


Table 5-1. Facilities that Produce, Process, or Use Phenol

\begin{tabular}{|c|c|c|c|c|}
\hline State $^{a}$ & $\begin{array}{l}\text { Number of } \\
\text { facilities }\end{array}$ & $\begin{array}{l}\text { Minimum } \\
\text { amount on site } \\
\text { in pounds }\end{array}$ & $\begin{array}{l}\text { Maximum } \\
\text { amount on site } \\
\text { in pounds }\end{array}$ & Activities and uses $^{c}$ \\
\hline$\overline{P R}$ & 15 & 0 & 999,999 & $1,2,3,4,5,6,7,8,9,10,12,13$ \\
\hline $\mathrm{RI}$ & 5 & 100 & 99,999 & $6,7,8$ \\
\hline SC & 53 & 0 & $49,999,999$ & $1,2,3,4,5,6,7,8,9,10,11,12,13,14$ \\
\hline SD & 1 & 1,000 & 9,999 & $3,6,10,11$ \\
\hline $\mathrm{TN}$ & 72 & 0 & $9,999,999$ & $1,2,3,4,5,6,7,8,9,10,11,12,13,14$ \\
\hline TX & 155 & 0 & $10,000,000,000$ & $1,2,3,4,5,6,7,8,9,10,11,12,13,14$ \\
\hline UT & 25 & 0 & 999,999 & $1,2,3,4,5,6,7,8,9,10,11,12,13$ \\
\hline VA & 41 & 0 & $49,999,999$ & $1,2,3,5,6,7,8,10,11,12,13,14$ \\
\hline VI & 4 & 100 & 999,999 & $1,2,3,4,5,6,7,9$ \\
\hline VT & 6 & 1,000 & 9,999 & $2,3,6,8,10$ \\
\hline WA & 58 & 0 & $9,999,999$ & $1,2,3,4,5,6,7,8,9,10,11,12,13,14$ \\
\hline WI & 60 & 0 & $9,999,999$ & $1,2,3,5,6,7,8,9,10,11,12,13$ \\
\hline WV & 27 & 0 & 49,999,999 & $1,2,3,4,5,6,7,8,9,10,13$ \\
\hline WY & 13 & 0 & 999,999 & $1,3,4,5,6,7,12,13$ \\
\hline
\end{tabular}

${ }^{\text {a }}$ post office state abbreviations used

${ }^{\mathrm{b}}$ Amounts on site reported by facilities in each state ${ }^{\mathrm{c} A c t i v i t i e s / \text { Uses: }}$
1. Produce
6. Impurity
11. Chemical Processing Aid
2. Import
7. Reactant
12. Manufacturing Aid
3. Onsite use/processing
8. Formulation Component
13. Ancillary/Other Uses
4. Sale/Distribution
9. Article Component
14. Process Impurity
5. Byproduct
10. Repackaging

Source: TRI05 2007 (Data are from 2005) 
Table 5-2. Current U.S. Producers of Phenol

\begin{tabular}{llc}
\hline Company & Location & $\begin{array}{c}\text { Capacity } \\
\text { (millions of pounds) }\end{array}$ \\
\hline Dakota Gasification Company & Beulah, North Dakota & 35 \\
The Dow Chemical Company & Oyster Creek, Texas & 650 \\
General Electric Company, GE Advanced & Mount Vernon, Indiana & 750 \\
Materials Plastics & & \\
Georgia Gulf Corporation & Pasadena, Texas & 160 \\
Georgia Gulf Corporation & Plaquemine, Louisiana & 500 \\
INOES Phenol Inc. & Theodore, Alabama & 1200 \\
JLM Chemicals & Blue Island, Illinois & 100 \\
Merisol USA LLC & Houston, Texas & 25 \\
Noveon Inc. & Kalama, Washington & 75 \\
Shell Chemical Company & Deer Park, Texas & 1,180 \\
Sunoco Inc. & Haverhill, Ohio & 1,000 \\
Sunoco Inc. & Philadelphia, Pennsylvania & 1,115 \\
\hline
\end{tabular}

Source: Derived from SRI 2006 


\subsection{DISPOSAL}

Phenol is listed as a toxic substance under Section 313 of the Emergency Planning and Community Right to Know Act (EPCRA) under Title III of the Superfund Amendments and Reauthorization Act (SARA) (EPA 1998c). Disposal of wastes containing phenol is controlled by a number of federal regulations (see Chapter 8). 
5. PRODUCTION, IMPORT/EXPORT, USE, AND DISPOSAL

This page is intentionally blank. 


\section{POTENTIAL FOR HUMAN EXPOSURE}

\subsection{OVERVIEW}

Phenol has been identified in at least 595 of the 1,678 hazardous waste sites that have been proposed for inclusion on the EPA National Priorities List (NPL) (HazDat 2006). However, the number of sites evaluated for phenol is not known. The frequency of these sites can be seen in Figure 6-1. Of these sites, 589 are located within the United States and 4 are located in the Commonwealth of Puerto Rico (not shown) and 2 are located in the Virgin Islands (not shown).

Phenol is released to the air and water as a result of its manufacture, its use in phenolic resins, and organic synthesis (Wallace et al. 1996). Phenol is found in petroleum products such as coal tar, and creosote and can be released by combustion of wood and auto exhaust (Wallace et al. 1996). Phenol is also produced by the natural degradation of organic wastes including benzene. Phenol is a major metabolite of benzene (Rothman et al. 1998), which is found extensively in the environment (Agency for Toxic Substances and Disease Registry 2006), therefore, phenol may be formed in the environment as a result of the natural degradation of benzene.

Phenol mainly enters the water from industrial effluent discharges. Phenol is degraded rapidly in air by gas-phase hydroxyl radical reaction (estimated half-life 14.6 hours), but may persist in water for a somewhat longer period. Half-lives for biodegradation range from $<1$ day in samples of lake water to 9 days in estuarine water; a typical half-life for photooxidation by photochemically produced peroxyl radicals is approximately 19 hours. In soil, phenol will generally biodegrade rapidly; however, biodegradation of phenol in water or soil may be hindered or precluded by the presence of high, toxic concentrations of phenol or other chemicals, or by other factors such as a lack of nutrients or microorganisms capable of degrading phenol. If biodegradation is sufficiently slow, phenol in sunlit water will undergo photooxidation with photochemically produced peroxyl radicals, and phenol in soil will leach to groundwater. Phenol may remain in air, water, and soil for much longer periods if it is continually or consistently released to these media from point sources. Since plants can metabolize phenol readily, exposure through eating food derived from plants grown in phenol-containing soil is probably minimal.

Phenol has been measured in effluents (up to $53 \mathrm{ppm}$ ), ambient water (1.5->100 ppb), drinking water (not quantified), groundwater (1.9->10 ppb), rain (0.075-1.2 ppb), sediment (>10 ppb), and ambient air (0.03$44 \mathrm{ppb})$. Occupational exposures occur through inhalation and dermal exposure; air concentrations 
Figure 6-1. Frequency of NPL Sites with Phenol Contamination
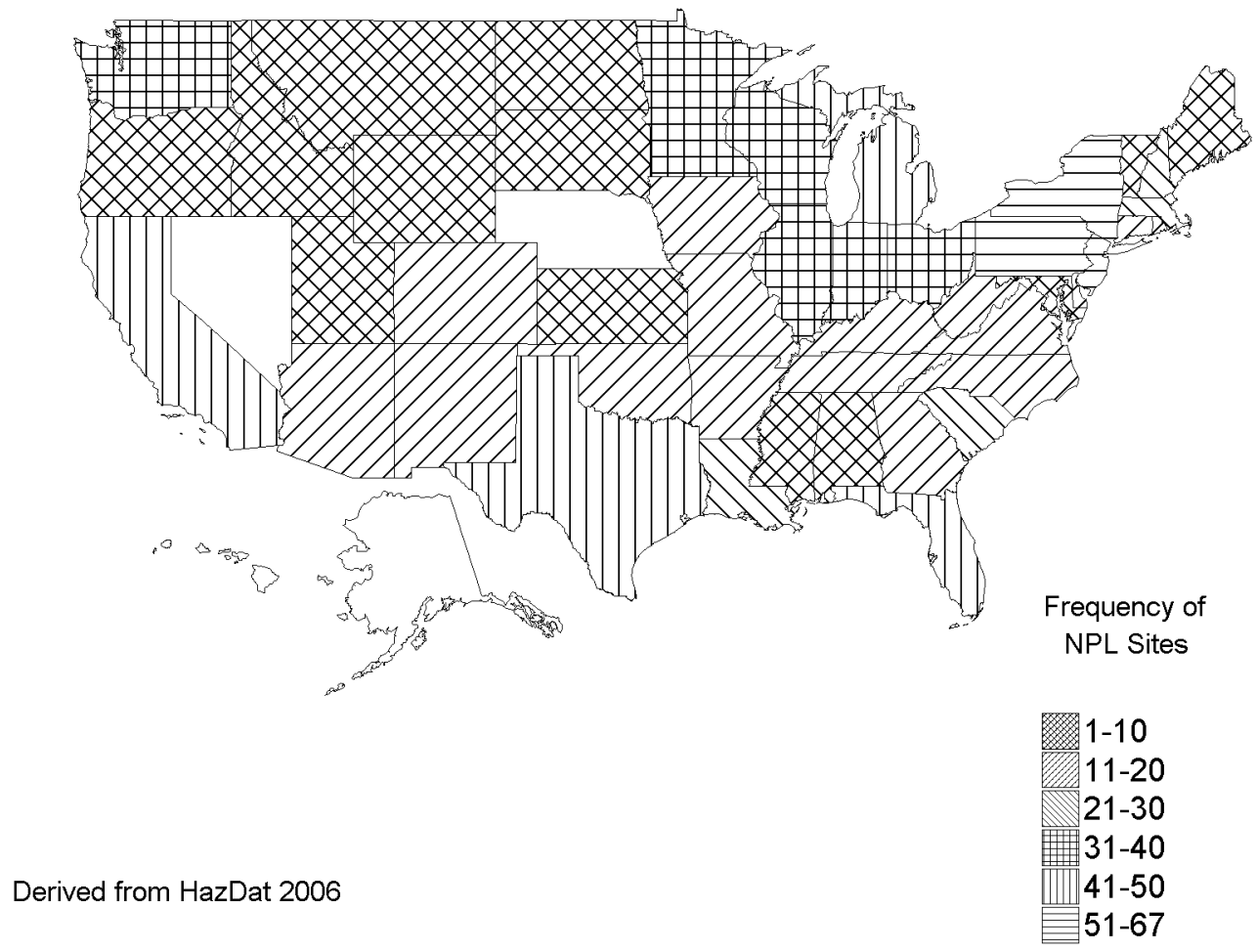
monitored in various workplaces range from 0.1 to $12.5 \mathrm{mg} / \mathrm{m}^{3}(0.03-32 \mathrm{ppm})$. Occupational as well as consumer exposure may also occur through dermal contact with phenol or phenol-containing products.

\subsection{RELEASES TO THE ENVIRONMENT}

The Toxics Release Inventory (TRI) data should be used with caution because only certain types of facilities are required to report (EPA 2005). This is not an exhaustive list. Manufacturing and processing facilities are required to report information to the TRI only if they employ 10 or more full-time employees; if their facility is included in Standard Industrial Classification (SIC) Codes 10 (except 1011, 1081, and 1094), 12 (except 1241), 20-39, 4911 (limited to facilities that combust coal and/or oil for the purpose of generating electricity for distribution in commerce), 4931 (limited to facilities that combust coal and/or oil for the purpose of generating electricity for distribution in commerce), 4939 (limited to facilities that combust coal and/or oil for the purpose of generating electricity for distribution in commerce), 4953 (limited to facilities regulated under RCRA Subtitle C, 42 U.S.C. section 6921 et seq.), 5169, 5171, and 7389 (limited S.C. section 6921 et seq.), 5169, 5171, and 7389 (limited to facilities primarily engaged in solvents recovery services on a contract or fee basis); and if their facility produces, imports, or processes $\geq 25,000$ pounds of any TRI chemical or otherwise uses $>10,000$ pounds of a TRI chemical in a calendar year (EPA 2005).

\subsubsection{Air}

Estimated releases of 7.6 million pounds ( $\sim 3,480$ metric tons) of phenol to the atmosphere from 677 domestic manufacturing and processing facilities in 2004 , accounted for about $64 \%$ of the estimated total environmental releases from facilities required to report to the TRI (TRI05 2007). These releases are summarized in Table 6-1.

During manufacturing, phenol is released primarily to the atmosphere from storage tank vents and during transport loading (EPA 1979c). Other major sources of release to the atmosphere are residential wood burning and automobile exhaust (EPA 1981a). Volatilization from environmental waters and soils has been shown to be a slow process (see Section 6.3.1) and is not expected to be a significant source of atmospheric phenol. Phenol is released into the atmosphere from industrial combustion processes. For example, phenol has been detected at a concentration of $0.36 \mathrm{ppb}$ in the emissions of a waste incinerator plant in Germany (Jay and Stieglitz 1995). In Brazil, an estimated $64 \mathrm{~kg} /$ year of phenol is released to the urban areas located near coal-fired power stations, indicating that people who live near coal-fired power stations may have an increased risk of exposure to phenol (Moreira dos Santos et al. 2004). Phenol is also 
Table 6-1. Releases to the Environment from Facilities that Produce, Process, or Use Phenol ${ }^{\mathrm{a}}$

\begin{tabular}{|c|c|c|c|c|c|c|c|c|c|}
\hline \multirow[b]{3}{*}{ State $^{\mathrm{c}}$} & \multirow[b]{3}{*}{$\mathrm{RF}^{\mathrm{d}}$} & \multicolumn{8}{|c|}{ Reported amounts released in pounds per year ${ }^{b}$} \\
\hline & & \multirow[b]{2}{*}{ Air ${ }^{\mathrm{e}}$} & \multirow[b]{2}{*}{ Water $^{f}$} & \multirow[b]{2}{*}{$\mathrm{UI}^{\mathrm{g}}$} & \multirow[b]{2}{*}{ Land $^{\mathrm{h}}$} & \multirow[b]{2}{*}{ Other ${ }^{i}$} & \multicolumn{3}{|c|}{ Total release } \\
\hline & & & & & & & On-site & Off-site & $\begin{array}{l}\text { On- and } \\
\text { off-site }\end{array}$ \\
\hline$\overline{\mathrm{AL}}$ & 33 & 407,745 & 278 & 0 & 8,213 & 0 & 408,261 & 7,975 & 416,236 \\
\hline AR & 15 & 53,803 & 136 & 0 & 4,781 & 0 & 54,241 & 4,479 & 58,720 \\
\hline$A Z$ & 2 & 5,499 & No data & 0 & 100 & 0 & 5,499 & 100 & 5,599 \\
\hline $\mathrm{CA}$ & 33 & 100,135 & 1,974 & 0 & 5,544 & 305 & 102,117 & 5,841 & 107,958 \\
\hline $\mathrm{CO}$ & 2 & 32 & No data & 0 & 0 & 0 & 32 & 0 & 32 \\
\hline $\mathrm{CT}$ & 4 & 5,631 & No data & 0 & 649 & 728 & 5,631 & 1,377 & 7,008 \\
\hline DE & 2 & 160 & 44,595 & 0 & 0 & 0 & 44,755 & 0 & 44,755 \\
\hline $\mathrm{FL}$ & 12 & 114,230 & 950 & 0 & 91 & 0 & 115,272 & 0 & 115,272 \\
\hline GA & 25 & 146,687 & 518 & 0 & 1,468 & 2,102 & 147,242 & 3,533 & 150,775 \\
\hline IA & 5 & 17,126 & 36 & 0 & 100,501 & 0 & 17,162 & 100,501 & 117,663 \\
\hline ID & 2 & 1,005 & 5 & 0 & 4,428 & 0 & 1,010 & 4,428 & 5,438 \\
\hline IL & 28 & 614,954 & 602 & 0 & 50,925 & 251 & 616,526 & 50,206 & 666,732 \\
\hline IN & 32 & 391,396 & 3,660 & 100,000 & 35,975 & 5 & 495,190 & 35,845 & 531,035 \\
\hline KS & 7 & 76,565 & 54 & 0 & 5,869 & 1,829 & 76,637 & 7,680 & 84,317 \\
\hline KY & 13 & 108,867 & 809 & 0 & 56,542 & 0 & 109,676 & 56,542 & 166,218 \\
\hline LA & 36 & 124,638 & 15,173 & 150,002 & 10,660 & 120 & 295,401 & 5,192 & 300,593 \\
\hline MA & 6 & 15,108 & No data & 0 & 803 & 0 & 15,108 & 803 & 15,911 \\
\hline MD & 2 & 13,004 & No data & 0 & 0 & 0 & 13,004 & 0 & 13,004 \\
\hline ME & 5 & 25,852 & 15 & 0 & 906 & 0 & 25,893 & 880 & 26,773 \\
\hline MI & 29 & 121,520 & 660 & 19 & 78,038 & 44,762 & 122,188 & 122,810 & 244,998 \\
\hline MN & 8 & 55,892 & 150 & 0 & 98 & 3 & 56,117 & 26 & 56,143 \\
\hline MO & 9 & 15,024 & 0 & 0 & 0 & 0 & 15,024 & 0 & 15,024 \\
\hline MS & 12 & 67,983 & 207 & 0 & 1,238 & 0 & 68,190 & 1,238 & 69,428 \\
\hline MT & 5 & 3,724 & 25 & 0 & 23 & 0 & 3,772 & 0 & 3,772 \\
\hline $\mathrm{NC}$ & 23 & 186,812 & 311 & 0 & 164,098 & 0 & 187,269 & 163,952 & 351,221 \\
\hline ND & 1 & 220 & 49 & 2,100 & 0 & 0 & 2,369 & 0 & 2,369 \\
\hline NE & 4 & 888 & No data & 0 & 147 & 0 & 896 & 139 & 1,035 \\
\hline $\mathrm{NH}$ & 2 & 6,203 & No data & 0 & 1 & 0 & 6,203 & 1 & 6,204 \\
\hline NJ & 10 & 6,218 & 1,828 & 0 & 10,327 & 0 & 16,022 & 2,351 & 18,373 \\
\hline NM & 1 & 1,078 & No data & 0 & 0 & 0 & 1,078 & 0 & 1,078 \\
\hline NY & 23 & 57,689 & 62 & 0 & 22,704 & 56,518 & 57,777 & 79,196 & 136,973 \\
\hline $\mathrm{OH}$ & 45 & 522,126 & 1,219 & 34 & 119,210 & 807 & 528,880 & 114,516 & 643,397 \\
\hline OK & 10 & 21,713 & 558 & 0 & 17,557 & 4,855 & 38,249 & 6,434 & 44,683 \\
\hline OR & 22 & 186,431 & 189 & 0 & 12,935 & 3,453 & 187,736 & 15,272 & 203,008 \\
\hline PA & 29 & 424,309 & 9,699 & 0 & 4,994 & 2,093 & 434,776 & 6,319 & 441,096 \\
\hline PR & 3 & 4,974 & No data & 0 & 4,561 & 0 & 4,974 & 4,561 & 9,535 \\
\hline RI & 1 & 724 & No data & 0 & 0 & 0 & 724 & 0 & 724 \\
\hline
\end{tabular}




\section{Table 6-1. Releases to the Environment from Facilities that Produce, Process, or Use Phenol ${ }^{\mathrm{a}}$}

\begin{tabular}{|c|c|c|c|c|c|c|c|c|c|c|}
\hline \multirow[b]{3}{*}{ State $^{c}$} & \multirow[b]{3}{*}{$\mathrm{RF}^{\mathrm{d}}$} & \multicolumn{9}{|c|}{ Reported amounts released in pounds per year ${ }^{b}$} \\
\hline & & \multirow[b]{2}{*}{ Air $^{\mathrm{e}}$} & \multirow[b]{2}{*}{ Water $^{f}$} & \multirow[b]{2}{*}{$\mathrm{UI}^{\mathrm{g}}$} & \multirow{2}{*}{\multicolumn{2}{|c|}{ Land $^{\mathrm{h}}$}} & \multirow[b]{2}{*}{ Other } & \multicolumn{3}{|c|}{ Total release } \\
\hline & & & & & & & & On-site & Off-site & $\begin{array}{l}\text { On- and } \\
\text { off-site }\end{array}$ \\
\hline$\overline{\mathrm{SC}}$ & 20 & 93,387 & 1,284 & & 0 & 148,473 & 0 & 115,489 & 127,656 & 243,145 \\
\hline TN & 15 & 81,725 & 305 & & 0 & 2,006 & 45 & 82,439 & 1,642 & 84,081 \\
\hline $\mathrm{TX}$ & 75 & 233,116 & 7,659 & $1,225,46$ & & 99,083 & 118 & $1,416,533$ & 148,909 & $1,565,442$ \\
\hline UT & 3 & 31,100 & No data & & 0 & 7,600 & 0 & 38,700 & 0 & 38,700 \\
\hline VA & 12 & 56,625 & 299 & & 0 & 31,225 & 750 & 87,264 & 1,635 & 88,899 \\
\hline VI & 1 & 78,848 & 612 & & 0 & 1 & 0 & 79,460 & 1 & 79,461 \\
\hline WA & 19 & 127,933 & 5,382 & & 0 & 427 & 181 & 133,355 & 568 & 133,923 \\
\hline WI & 23 & 305,630 & 55 & & 0 & 27,762 & 30 & 305,685 & 27,792 & 333,477 \\
\hline WV & 6 & 20,279 & 188 & & 0 & 69 & 44 & 20,475 & 105 & 20,580 \\
\hline WY & 2 & 750 & No data & & 0 & 0 & 0 & 750 & 0 & 750 \\
\hline Total & 677 & $4,935,360$ & 99,546 & $1,477,62$ & & $1,040,033$ & 118,999 & $6,561,054$ & $1,110,505$ & $7,671,559$ \\
\hline
\end{tabular}

${ }^{a}$ The TRI data should be used with caution since only certain types of facilities are required to report. This is not an exhaustive list. Data are rounded to nearest whole number.

${ }^{b}$ Data in TRI are maximum amounts released by each facility.

${ }^{\mathrm{C}}$ Post office state abbreviations are used.

${ }^{\mathrm{d}}$ Number of reporting facilities.

${ }^{\mathrm{e}}$ The sum of fugitive and point source releases are included in releases to air by a given facility.

fSurface water discharges, waste water treatment-(metals only), and publicly owned treatment works (POTWs) (metal and metal compounds).

${ }^{\mathrm{g}}$ Class I wells, Class II-V wells, and underground injection.

${ }^{\mathrm{h}}$ Resource Conservation and Recovery Act (RCRA) subtitle C landfills; other on-site landfills, land treatment, surface impoundments, other land disposal, other landfills.

'Storage only, solidification/stabilization (metals only), other off-site management, transfers to waste broker for disposal, unknown

The sum of all releases of the chemical to air, land, water, and underground injection wells.

kTotal amount of chemical transferred off-site, including to POTWs.

$\mathrm{RF}=$ reporting facilities; $\mathrm{UI}=$ underground injection

Source: TRI05 2007 (Data are from 2005) 
found in cigarette smoke and in plastics (Graedel 1978), but no data are available to determine the extent of exposure to phenol from these sources.

\subsubsection{Water}

Estimated releases of 99,546 pounds ( $\sim 45$ metric tons) of phenol to surface water from 677 domestic manufacturing and processing facilities in 2005 , accounted for about $0.1 \%$ of the estimated total environmental releases from facilities required to report to the TRI (TRI05 2007). These releases are summarized in Table 6-1.

The most common anthropogenic sources of phenol in natural water include coal tar (Thurman 1982) and waste water from manufacturing industries such as resins, plastics, fibers, adhesives, iron, steel, aluminum, leather, rubber (EPA 1981b), and effluents from synthetic fuel manufacturing (Parkhurst et al. 1979). Phenol is also released from paper pulp mills (Keith 1976) and wood treatment facilities (Goerlitz et al. 1985). Other releases of phenol result from commercial use of phenol and phenol-containing products, including slimicides, general disinfectants (Budavari et al. 1989; Hawley 1981), and medicinal preparations such as throat lozenges, mouthwashes, gargles, and antiseptic lotions (Darisimall 2006). Two natural sources of phenol in aquatic media are animal wastes and decomposition of organic wastes (EPA 1980). As a metabolite of benzene, phenol may be released from publicly owned treatment works (POTWs) and sewage overflow. For example, it has been estimated that $3.8 \mathrm{~kg} /$ day of phenol are released to Newark Bay, in New Jersey, from municipal treatment facilities (Crawford et al. 1995). Phenol was detected in $2 \%$ of the effluent samples from New York City sewage waste facilities collected during 1989 to 1993 at concentrations of 6-310 g/L (Stubin et al. 1996). Because it is a metabolite, phenol is likely to be found in other sewage and POTWs facilities. No data are available to determine the extent of exposure from these sources.

Estimated releases of 85,700 pounds ( $\sim 39$ metric tons) of phenol to surface water from 689 domestic manufacturing and processing facilities in 2004 , accounted for about $0.1 \%$ of the estimated total environmental releases from facilities required to report to the TRI (TRI05 2007). These releases are summarized in Table 6-1.

The most common anthropogenic sources of phenol in natural water include coal tar (Thurman 1982) and waste water from manufacturing industries such as resins, plastics, fibers, adhesives, iron, steel, aluminum, leather, rubber (EPA 1981b), and effluents from synthetic fuel manufacturing (Parkhurst et al. 
1979). Phenol is also released from paper pulp mills (Keith 1976) and wood treatment facilities (Goerlitz et al. 1985). Other releases of phenol result from commercial use of phenol and phenol-containing products, including slimicides, general disinfectants (Budavari et al. 1989; Hawley 1981), and medicinal preparations such as throat lozenges, mouthwashes, gargles, and antiseptic lotions (Darisimall 2006).

\subsubsection{Soil}

Estimated releases of 1 million pounds ( 472 metric tons) of phenol to soils from 689 domestic manufacturing and processing facilities in 2005, accounted for about $14 \%$ of the estimated total environmental releases from facilities required to report to the TRI (TRI05 2007). An additional 1.5 million pounds ( $\sim 670$ metric tons), constituting about $19 \%$ of the total environmental emissions, were released via underground injection (TRI05 2007). These releases are summarized in Table 6-1.

Phenol may be released to the soil during its manufacturing process, when spills occur during loading and transport, and when it leaches from hazardous waste sites and landfills (Xing et al. 1994). Generally, data on concentrations of phenol found in soil at sites other than hazardous waste sites are lacking. This may be due in part to a rapid rate of biodegradation and leaching (see Sections 6.3.1 and 6.3.2.3). Phenol can be expected to be found in soils that receive continuous or consistent releases from a point source. Phenol that leaches through soil to groundwater spends at least some time in that soil as it travels to the groundwater. Phenol has been found in groundwater, mainly at or near hazardous waste sites.

\subsection{ENVIRONMENTAL FATE}

\subsubsection{Transport and Partitioning}

Phenol is released into the air and discharged into water from both manufacturing and use. Based on its high water solubility (see Table 4-2) and the fact that it has been detected in rainwater, some phenol may wash out of the atmosphere; however, it is probable that only limited amounts wash out because of the short atmospheric half-life of phenol. During the day, when photochemically produced hydroxyl radical concentrations are highest in the atmosphere, very little atmospheric transport of phenol is likely to occur.

In water, neither volatilization nor sorption to sediments and suspended particulates are expected to be important transport mechanisms. Using the Henry's Law constant of $3 \times 10^{-7} \mathrm{~atm} \mathrm{~m}^{3} / \mathrm{mol}$ (Gaffney et al. 1987), a volatilization half-life of 88 days was calculated for phenol evaporation from a model river $1 \mathrm{~m}$ deep with a current of $1 \mathrm{~m} / \mathrm{second}$, and wind velocity of $3 \mathrm{~m} /$ second (Lyman et al. 1982). The biological 
treatment of waste water containing phenol has shown that $<1 \%$ of phenol is removed by stripping (Kincannon et al. 1983; Petrasek et al. 1983).

Phenol has been reported in sediments at levels as high as 608 ppm dry weight; however, it is not known whether the location of the site where this concentration was reported is at or near a point source of release, such as a hazardous waste dump. The concentrations of the overlying waters were not reported. The moderately low soil sorption partition coefficient (1.21-1.96) suggests that sorption to sediment is not an important transport process. There is very little sorption of phenol onto aquifer materials (Ehrlich et al. 1982), suggesting that phenol sorption to sediments may also be minimal. Based on the soil adsorption coefficient, phenol is expected to leach to groundwater; however, the rate of phenol biodegradation in the soil may be so rapid, except in cases of large releases such as spills or continuous releases such as leaching from landfill sites, that the probability of groundwater contamination may be low (Ehrlich et al. 1982). Phenol has been detected in groundwater as a result of leaching through soil from a spill of phenol (Delfino and Dube 1976), from landfill sites (Clark and Piskin 1977), and from hazardous waste sites (Plumb 1987). The sorption coefficient for phenol by soils increases with increasing soil organic matter which may indicate that soil organic matter may be the primary phenol sorbent in soil (Xing et al. 1994).

Phenol is not expected to bioconcentrate significantly in aquatic organisms. Reported log bioconcentration factors (BCF) in fish for phenol include 0.28 for goldfish, (Kobayashi et al. 1979) and 1.3 for golden orfe (Freitag et al. 1984). The highest mean level of phenol detected in bottom fish from Commencement Bay in Tacoma, Washington, was $0.14 \mathrm{ppm}$ (Nicola et al. 1987). The levels of phenol in the water or sediments were not stated.

The pKa of phenol is 10 (O'Neil 2001), indicating that phenol will primarily exist as the protonated acid at environmental $\mathrm{pH}$ values. In alkaline soils and water, phenol will partially exist as an anion, which can affect its fate and transport processes.

Although it has been shown that plants readily uptake phenol (DOE 1987), bioaccumulation does not take place due to a high rate of respiratory decomposition of phenol to $\mathrm{CO}_{2}$. 


\subsubsection{Transformation and Degradation}

\subsubsection{Air}

The gas-phase reaction of phenol with photochemically produced hydroxyl radicals is probably a major removal mechanism in the atmosphere. An estimated half-life for phenol for this reaction is 0.61 days (EPA 1979a). The reaction of phenol with nitrate radicals during the night may constitute a significant removal process. This is based on a rate constant of $3.8 \times 10^{-12} \mathrm{~cm}^{3} /$ molecule second for this reaction, corresponding to a half-life of 15 minutes at an atmospheric concentration of $2 \times 10^{8}$ nitrate radicals per $\mathrm{cm}^{3}$ (Atkinson et al. 1987). The reaction of phenol with nitrate radicals present in the atmosphere during smog episodes may decrease the half-life of phenol in polluted atmospheres. The above data indicate that phenol has a short half-life in the atmosphere, probably $<1$ day. Phenol does not absorb light in the region of 290-330 nm (Lide and Milne 1994); therefore, it should not photodegrade directly in the atmosphere.

\subsubsection{Water}

Although phenol does not absorb light at wavelengths $>290$, phenols react rapidly to sunlit natural water via an indirect reaction with photochemically produced hydroxyl radicals and peroxyl radicals; typical half-lives for hydroxyl and peroxyl radical reactions are on the order of 100 and 19.2 hours of sunlight, respectively (Canonica et al. 1995; Mill and Mabey 1985). These reactions require dissolved natural organic materials that function as photosensitizers (Canonica et al. 1995). The estimated half-life for the reaction of phenol with photochemically produced singlet oxygen in sunlit surface waters contaminated by humic substances is 83 days (assuming Switzerland summer sunlight and a singlet oxygen concentration of $4 \times 10^{-14}$ molar [M]) (Scully and Hoigne 1987).

Phenol is readily biodegradable in natural water, provided the concentration is not high enough to cause significant inhibition through microbial toxicity. Complete degradation in $<1$ day has been reported in water from three lakes; the rates of degradation were affected by the concentration of organic and inorganic nutrients in the water (Rubin and Alexander 1983). Complete removal of phenol in river water has been reported after 2 days at $20^{\circ} \mathrm{C}$ and after 4 days at $4{ }^{\circ} \mathrm{C}$ (Ludzack and Ettinger 1960). The degradation of phenol is somewhat slower in salt water, and a half-life of 9 days has been reported in an estuarine river (EPA 1979b). Rapid degradation of phenol also has been reported in various sewage and water treatment processes. Removal in aerobic activated sludge reactors is frequently $>90 \%$ with a retention time of 8 hours (Stover and Kincannon 1983). In aerobic reactors using municipal seed 
(conventional activated sludge organisms) and in reactors using an industrial seed (mixture of organisms), it was noted that concentrations as low as $50 \mathrm{mg} / \mathrm{L}$ inhibited organism respiration rates, but complete inhibition was not observed at concentrations as high as $200 \mathrm{mg} / \mathrm{L}$ (Davis et al. 1981). Utilization is also very high in anaerobic reactors, although acclimation periods are longer and degradation usually takes about 2 weeks (Boyd et al. 1983; Healy and Young 1978). One method of phenol breakdown is accomplished by the bacterium Pseudomonas sp. CF600, which uses a set of enzymes encoded by the plasmid dmp operon (Powlowski and Shingler 1994). The use of sequence batch reactors (SBR) in treating sludge contaminated with phenolic compounds has proven effective in breaking down the compounds biologically with no evidence of phenol volatility (Al-Harazin et al. 1991). Levels as high as a one-time treatment of $1,600 \mathrm{mg} / \mathrm{L}$ can be broken down by $75 \%$ with a 1-day retention time. Lower concentrations as high as $800 \mathrm{mg} / \mathrm{L}$ can be broken down to $<0.5 \mathrm{mg} / \mathrm{L}$ with a 1-day retention time. The alga Ochromonas danica has also been shown to degrade phenol (Semple and Cain 1996). When grown in the dark with 0.1-1 mM phenol as the sole carbon source, phenol was removed within 3 days. Because of the rapid rate of biodegradation, groundwater is generally free of phenol even though it is highly mobile in soil. However, monitoring data in Section 6.4.2 contain groundwater concentrations in areas of large phenol releases.

While the evidence presented in the literature cited above suggests that phenol can be rapidly and virtually completely degraded under both natural water and sewage treatment plant conditions, monitoring data presented in Section 6.4 below indicate that phenol, despite this apparent biodegradability, is still present in the environment. This suggests that the exact conditions under which phenol is rapidly degraded are not present in all instances. In some situations, the concentration of phenol may be too high or the populations of microorganisms may not be present in sufficient concentration for significant biodegradation to occur.

\subsubsection{Sediment and Soil}

Available data indicate that phenol biodegrades in soil under both aerobic and anaerobic soil conditions. The half-life of phenol in soil is generally $<5$ days (Baker and Mayfield 1980), but acidic soils and some surface soils may have half-lives of up to 23 days (Shiu et al. 1994). Mineralization in an alkaline, parabrown soil under aerobic conditions was 45.5, 48, and 65\% after 3, 7, and 70 days, respectively (Haider et al. 1974). Half-lives for degradation of low concentrations of phenol in two silt loam soils were 2.70 and 3.51 hours (Scott et al. 1983). Plants have been shown to be capable of metabolizing phenol readily (DOE 1987). 
While degradation is slower under anaerobic conditions, evidence presented in the literature suggests that phenol can be rapidly and virtually completely degraded in soil under both aerobic and anaerobic conditions (Baker and Mayfield 1980).

\subsection{LEVELS MONITORED OR ESTIMATED IN THE ENVIRONMENT}

Reliable evaluation of the potential for human exposure to phenol depends in part on the reliability of supporting analytical data from environmental samples and biological specimens. Concentrations of phenol in unpolluted atmospheres and in pristine surface waters are often so low as to be near the limits of current analytical methods. In reviewing data on phenol levels monitored or estimated in the environment, it should also be noted that the amount of chemical identified analytically is not necessarily equivalent to the amount that is bioavailable. The analytical methods available for monitoring phenol in a variety of environmental media are detailed in Chapter 7.

\subsubsection{Air}

There are very few monitoring data concerning the presence of phenol in ambient air. Phenol was found at a median concentration of 30 parts per trillion (ppt) in 7 samples from 1 U.S. urban/suburban site in 1974 and at an overall median concentration of 5,000 ppt in 83 samples from 7 source-dominated sites between 1974 and 1978 (EPA 1982). The individual medians of the seven source sites ranged from 520 to 44,000 ppt (EPA 1982). Phenol was detected, but not quantified, in air above the Niagara River in September of 1982 (Hoff and Chan 1987). Phenol concentrations in two urban areas ranged from 13 to $91 \mathrm{ppt}$ and from $<5$ to $75 \mathrm{ppb}$ with $50 \%$ of all measurements $<8 \mathrm{ppb}$ (EPA 1981a). Phenol was found at approximately $1 \mathrm{ppb}$ in the ambient air near a fishmeal factory in Japan (Hoshika et al. 1981). In a study to determine contaminants in the air of agricultural areas, phenol was found in 42 out of 53 air samples taken from 8 farms at an average concentration of $10 \mu \mathrm{g} / \mathrm{m}^{3}$ (Sunesson et al. 2001). Phenol was detected in indoor and outdoor air in the city of Ottawa, Canada at concentration ranges of 0.01-5.16 and 0.01$1.41 \mu \mathrm{g} / \mathrm{m}^{3}$, respectively (Zhu et al. 2005).

In a study of various air samples from Helsinki, Finland, $92 \%$ of outdoor air and $86 \%$ of indoor air did not have detectible quantities of phenol (Edwards et al. 2001). Workplace and personal exposure samples also had low relative percentages of phenol with only 12 and $11 \%$ of the samples above the limits of detection, respectively (Edwards et al. 2001). 
Higher phenol concentrations may occur when there is smog or in highly contaminated air. During a smog episode in West Covina, California, in July of 1973, phenol concentrations ranged from 16 to 91 ppt, with a mean concentration of 60 ppt (Cronn et al. 1977). During a haze episode in 1998 in Brunei, Syria, phenol was found in 8 out of 15 sites tested in concentrations ranging from 0.07 to $0.41 \mu \mathrm{g} / \mathrm{m}^{3}$; of these sites, 4 were near a hospital (Muraleedharan et al. 2000).

Phenol and other volatile organic compounds were measured in the air of 50 homes or apartments in Finland (Kostiainen 1995). The average concentration was $0.23 \mathrm{ppb}$, with a range of $0-0.77 \mathrm{ppb}$. Phenol levels were not significantly higher in houses in which people complained of symptoms that resembled those of a sick building syndrome.

Phenol has been detected in the ash phase of burning pine, oak, and eucalyptus in concentrations of 524, 300 , and $434 \mathrm{mg} / \mathrm{kg}$, respectively (Schauer et al. 2001). Phenol has also been identified in the combustion of soft fireplace wood, hard fireplace wood, stove wood, and synthetic wood at concentrations of 158 , 247, 142, and $8.58 \mathrm{mg} / \mathrm{kg}$, respectively (McDonald et al. 2000).

Phenol is released to the atmosphere from burning coal. In a study of three urban sites in Brazil near coal-fired power stations, phenol was detected in all three locations with concentrations ranging from 0.98 to $1.60 \mu \mathrm{g} / \mathrm{m}^{3}$ (Moreira dos Santos et al. 2004). This indicates that families living near coal-fired power stations may be exposed to higher concentrations of phenol.

\subsubsection{Water}

Phenol has been detected in surface waters, rainwater, sediments, drinking water, groundwater, industrial effluents, urban runoff, and at hazardous waste sites. Background levels of phenol from relatively pristine sites can be as high as $1 \mathrm{ppb}$ for unpolluted groundwater and have been reported to range from 0.01 to $1 \mathrm{ppb}$ in unpolluted rivers (Thurman 1985). Phenol has been detected in Lake Huron water at 3-24 ppb (Konasewich et al. 1978) and industrial rivers in the United States at 0-5 ppb (Sheldon and Hites 1978, 1979). The annual mean concentration of phenol in water from the lower Mississippi River was $1.5 \mathrm{ppb}$ (EPA 1980). River water in an unspecified location in the United States was reported to contain 10$100 \mathrm{ppb}$ of phenol (Jungclaus et al. 1978). Phenol was detected, but not quantified, in a Niagara River watershed (Elder et al. 1981) and in 2 of 110 raw water samples analyzed during the National Organic Monitoring Survey (EPA 1980). 
In a study of 95 organic waste components from 139 streams in 30 states from 1999 to 2000, phenol was detected in 85 samples with a median concentration of $0.7 \mu \mathrm{g} / \mathrm{L}$ (Kolpin et al. 2002). Phenol has been detected in China in water samples taken near the Huanchao River water factory, in the Tanking River, and near the Tanking River water factory at concentrations of $0.053,0.044$, and $0.033 \mu \mathrm{g} / \mathrm{L}$, respectively (Huang et al. 2003).

Each year about 30 million $\mathrm{m}^{3}$ of mine water is pumped into the Samara River in the Ukraine. Phenol was detected in the surface water in the Samara River from 1987 to 1990 with concentrations ranging from 1.5 to $8.3 \mu \mathrm{g} / \mathrm{L}$ (Goncharuk and Milyukin 1999). In a study of organic contaminants in the groundwater from creosote contaminated sites in Denmark, phenol was detected in all 11 sites tested with concentrations ranging from 11 to $249 \mu \mathrm{g} / \mathrm{L}$ (Johansen et al. 1997).

In general, higher levels of phenol appear to be found in lakes and rivers that serve as water sources and discharge receivers for industrial and population centers, probably as a result of industrial activity and commercial use of phenol-containing products. For example, the presence of higher levels of phenol in the Delaware River near Philadelphia is the result of industrial effluents discharged into the sewer system (Sheldon and Hites 1979). Phenol was detected in 23\% of samples of influent of waste water from New York City during 1989 to 1993 at concentrations ranging from 8 to $490 \mathrm{~g} / \mathrm{L}$; phenol was detected in 2\% of effluent samples at concentrations ranging from 6 to $310 \mathrm{~g} / \mathrm{L}$ during the same period (Stubin et al. 1996).

The presence of phenol in drinking water probably results from using contaminated surface water or groundwater as a source. Its presence in groundwater is probably the result of release to soil, often industrial releases or leachate from waste dumps, and the subsequent leaching of phenol through the soil to the groundwater. Phenol has been detected, but not quantified, in drinking water from 5 of 14 drinking water treatment plants between July 1977 and June 1979 in one of three sites (groundwater source), in three out of ten (surface water source) as well as water source used after distribution (Fielding et al. 1981). Phenol levels in tap water, spring water, and mineral water in Italy were $0.58,0.051$, and $0.161 \mu \mathrm{g} / \mathrm{L}$, respectively (Achilli et al. 1995). Phenol was detected at a maximum concentration of $1,130 \mathrm{ppm}$ in ninewells in Wisconsin after a spill, and was detected for at least 1.5 years after the spill (Delfino and Dube 1976). It was found at concentrations up to $10.4 \mathrm{ppm}$ in groundwater from a sand aquifer adjacent to waste ponds at a wood-preserving facility in Florida (Goerlitz et al. 1985), and was detected at $6.510,000 \mathrm{ppb}$ in two aquifers 15 months after the completion of a coal gasification project (Stuermer et al. 1982). Phenol was detected at a maximum concentration of $1.9 \mathrm{ppm}$ in leachates from landfill sites in Illinois (Clark and Piskin 1977). Near a landfill in central Florida, phenol was found in 
groundwater and surface water at about 17 and $15 \mathrm{ppb}$, respectively (Chen and Zoltek 1995). Phenol was detected, but not quantified, in the groundwater at 13.6\% of 178 CERCLA hazardous waste sites (Plumb 1987).

Phenol was detected during seven rain events in Portland, Oregon, between February and April of 1984. Concentrations in rain ranged from $>75$ to $1,200 \mathrm{ppt}$, and averaged above $280 \mathrm{ppt}$. Gas-phase concentrations ranged from 220 (56.1 ppt) to $410 \mathrm{ng} / \mathrm{m}^{3}$ (105 ppt) and averaged $320 \mathrm{ng} / \mathrm{m}^{3}$ (82 ppt) (Leuenberger et al. 1985).

Phenol can also be transported in snow and rain. In Germany, phenol has also been detected in clouds at Mount Brocken in June 1994 and during April to May in Great Dun Fell with mean concentrations of $3.0 \mu \mathrm{g} / \mathrm{L}$ and $5.4 \mu \mathrm{g} / \mathrm{L}$, respectively (Lüttke 1999). Snow from Neulaniemi, Muonio, and Levi, Finland, contained phenol in concentrations of $0.16,0.04$, and $0.02 \mu \mathrm{g} / \mathrm{kg}$, respectively (Poliakova et al. 2000). In Russia, phenol was detected in snow samples at concentrations of $0.02,0.21$, and $1.3 \mu \mathrm{g} / \mathrm{kg}$ in Butovo, Moscow State University, and Shosse Entuzoastov (a heavily industrial district in Moscow), respectively (Poliakova et al. 2000).

Phenol has been detected in the effluent discharges of a variety of industries. It was found in petroleum refinery waste water at concentrations of $33.5 \mathrm{ppm}$ (Pfeffer 1979) and $100 \mathrm{ppb}$ (Paterson et al. 1996), in the treated and untreated effluent from a coal conversion plant at 4 and 4,780 ppm, respectively (Parkhurst et al. 1979), and in shale oil waste water at a maximum of $4.5 \mathrm{ppm}$ (Hawthorne and Sievers 1984). It has also been detected in the effluent from a chemical specialties manufacturing plant at 0.01$0.30 \mathrm{ppm}$ (Jungclaus et al. 1978), in effluent from paper mills at 5-8 ppb (Keith 1976; Paterson et al. 1996), and at $0.3 \mathrm{ppm}$ in a 24-hour composite sample from a plant on the Delaware River, 2 and 4 miles downriver from a sewage treatment plant (Sheldon and Hites 1979).

A study of the seasonal effects on the concentration of pesticides as well as other contaminants in Gulf of Gdansk in the Baltic Sea from autumn 2001 to spring 2003 showed only 1 out of 14 samples taken in autumn to have a detectable level of phenol with a concentration of $2.3 \mu \mathrm{g} / \mathrm{L}$ (Kot-Wasik et al. 2004). In the spring, phenol was detected in 11 of the 14 samples at concentrations ranging from 0.3 to $0.8 \mu \mathrm{g} / \mathrm{L}$ (Kot-Wasik et al. 2004).

Phenol has also been found in the primary and secondary effluent from the Los Angeles City Treatment Plant at concentrations of 32 and $<10 \mathrm{ppb}$, respectively (Young et al. 1983). It was found in 3 of 
86 samples of runoff from 2 of 15 cities at $3-10 \mathrm{ppb}$ by the U.S. Nationwide Urban Runoff Program as of July of 1982 (Cole et al. 1984).

\subsubsection{Sediment and Soil}

Very few data concerning the presence of phenol in soils were found. Phenol generally does not adsorb very strongly to soils and tends to leach rapidly through soil, which may account for the lack of monitoring data, since any phenol released to soils is likely to leach to groundwater. Moreover, phenol is readily degraded in the environment, which is expected to attenuate its levels in soil.

Sediment collected $6 \mathrm{~km}$ northwest of the Los Angeles County waste-water treatment plant discharge zone at Palos Verdes, California, contained 10 ppb (dry weight) phenol (Gossett et al. 1983).

In a study of contaminants in stream bed sediments across the United States, phenol was detected at 536 sites at a maximum concentration of $210 \mu \mathrm{g} / \mathrm{kg}$ (Lopes and Furlong 2001).

\subsubsection{Other Environmental Media}

Phenol has been reported at concentrations of 7 and $28.6 \mathrm{ppm}$ in smoked summer sausage and smoked pork belly, respectively (EPA 1980), and was identified but not quantified in mountain cheese (Dumont and Adda 1978), fried bacon (Ho et al. 1983), fried chicken (Tang et al. 1983), and black fermented tea (Kaiser 1967). Phenol has also been found in honey at concentrations ranging from 0 (detection limit $0.1 \mathrm{ppm}$ ) to $19 \mathrm{ppm}$ (Sporns 1981). It was present each time the honey was collected with phenol-treated boards. Phenol has been reported in three different types of fermented soybean curds at concentrations ranging from 450 to $6,000 \mu \mathrm{g} / \mathrm{kg}$ (Chung 1999).

Phenol has been found in bottomfish from five sites in Commencement Bay in Tacoma, Washington, at a highest maximum average and overall maximum concentration of 0.14 and $0.22 \mathrm{ppm}$, respectively (Nicola et al. 1987). Phenol has been reported to be a natural component of animal matter; it has been found at 0-1.6 ppm in rabbit muscle tissue (EPA 1980).

Phenol has been detected in the eggs of birds in the Lake Baikal Region of Siberia, Russia in concentrations ranging from 840 to $<20 \mu \mathrm{g} / \mathrm{kg}$ dry weight (Lebedev et al. 1998). 
Phenol is also found in medicinal preparations including throat lozenges, mouthwashes, gargles, and antiseptic lotions (Darisimall 2006). Commercial antiseptic lotions may contain up to $1.4 \%$ phenol (Darisimall 2006). Package labeling information indicates that commercial throat lozenges contain up to $29 \mathrm{mg}$ of phenol per lozenge (Darisimall 2006). Other consumer products such as disinfectants and cleaners may contain phenol (O'Neil 2001).. It has been found that the smoke of 1 nonfilter cigarette contains $60-140 \mu \mathrm{g}$ of phenol, 19-35 $\mu \mathrm{g}$ for a filter-tipped cigarette, and 24-107 $\mu \mathrm{g}$ in cigars (IARC 1986; NCI 1998). Using a testing method developed by the U.S. Federal Trade Commission (FTC), analysis of the smoke from several different brands and types of cigarettes found phenol in concentrations ranging from 0.3 to $68 \mu \mathrm{g} /$ cigarette (Roemer et al. 2004). The lowest concentration came from an electrically heated prototype cigarette, while most of the commercial brands were within a range of 2.6$23.4 \mu \mathrm{g} /$ cigarette (Roemer et al. 2004).

While not detected in topsoil from Holy Cross Mountain Park, Poland, and surrounding area, phenol was detected in 4 out of the 12 pine needle samples taken from the same area (Migaszewski 1999).

Phenol was detected in 303 of 389 samples of settled dust from indoor residences in Sweden; the mean concentration of phenol was $4 \mu \mathrm{g} / \mathrm{g}$ dust (Nilsson et al. 2005).

\subsection{GENERAL POPULATION AND OCCUPATIONAL EXPOSURE}

Data concerning concentrations of phenol in ambient air are insufficient to estimate the potential for exposure by inhalation. However, smoke from a single nonfilter cigarette was observed to contain 60 $140 \mu \mathrm{g}$ phenol, while levels of phenol range from 19 to $35 \mu \mathrm{g}$ in the smoke of filter-tipped cigarettes, and from 24 to $107 \mu \mathrm{g}$ in the smoke of cigars (IARC 1986; NCI 1998). Therefore, indoor environments polluted with tobacco smoke are likely to contain measurable amounts of phenol (Guerin et al. 1992). Nonsmokers who live with smokers are thought to be exposed to as much as $1.1 \mu \mathrm{g} / \mathrm{m}^{3}$. This would account for a daily inhalation of 6-14 $\mu \mathrm{g}$ /day (Nazaroff and Singer 2004).

Phenol concentrations in surface and drinking waters are expected to vary with location and proximity to varying industrial and municipal discharges. Considering the lack of quantitative, current monitoring data and the probable seasonal, spatial, and temporal variations in the concentrations of phenol at these sources, it is not possible to estimate accurately a potential daily dose of phenol from drinking contaminated water or from dermal exposure to contaminated water. Nonetheless, it is probable that only those systems that receive their water from contaminated surface water and groundwater contain phenol. 
Although phenol has been identified in various food products, a quantitative estimate regarding the intake of phenol from the ingestion of food has not been estimated.

Few data concerning occupational exposures to phenol were located. The average airborne concentrations of phenol to which workers were exposed at three locations within two wood creosote impregnation plants ranged from 0.03 to $0.5 \mathrm{ppm}$ (Heikkila et al. 1987). A phenol concentration of approximately $0.5 \mathrm{ppm}$ was measured in the workroom air at a casting factory in Osaka City, Japan (Kuwata et al. 1980), and concentrations as high as 3.2 ppm were measured in Japanese Bakelite factories (Ohtsuji and Ikeda 1972). Considering the lack of quantitative monitoring data for phenol in occupational atmospheres, it is not possible to estimate the potential for occupational exposure to phenol. The data, however, do show that exposure to phenol through breathing and dermal contact with contaminated workroom atmospheres is possible. The National Occupational Exposure Survey (NOES) conducted by NIOSH estimated that 584,372 workers were exposed to phenol in the United States (NIOSH 1990). The NOES database does not contain information on the frequency, concentration, or duration of exposures. The survey provides only estimates of workers potentially exposed to chemicals in the workplace.

Other occupations that may have increased exposure risk include laboratory workers, morticians, and house cleaners. Phenol is a common solvent used in laboratory research as well as a component of embalming fluid. The general population may be exposed to phenol found in consumer products such as general disinfectants used to clean toilets, floors, drains, and other areas (Lewis 2001; O'Neil 2001). House cleaners and people who work with these products daily are likely to have increased exposure.

Exposure to phenol also occurs through the use and subsequent ingestion of phenol-containing products, including mouthwashes, gargles, and throat sprays (Darisimall 2006). The concentration of phenol in throat sprays ranged from 0.5 to $1.4 \%$ phenol. Determining actual dosage from these sprays would be difficult as the delivery method is not precise. Cepastat ${ }^{\circledR}$ lozenges, a readily available remedy for sore throats, contains 14.5-29 mg phenol/lozenge (Darisimall 2006). If a patient (adults and children over 6) takes the maximum recommended daily number of 18 lozenges, this would result in approximate doses of 270-520 mg phenol/day. There is no control over the intake of non-prescription drugs and therefore, some individuals may consume considerably higher doses of phenol.

The estimated relative contributions of the various exposure routes and sources of total phenol exposure cannot be estimated using the available data. Nonetheless, for persons not exposed to phenol in the workplace, exposure will most likely result from: inhalation of contaminated ambient air, primarily in the 
vicinity of industries and municipalities that release significant amounts of phenol into the atmosphere; ingestion of drinking water from contaminated surface waters or groundwaters; ingestion of phenolcontaining products; and dermal exposure to contaminated water and to phenol-containing products. Dermal contact with phenol or ingestion of phenol-containing products probably constitutes the largest consumer exposure, although this exposure may occur on an acute basis. Inhalation and dermal exposures appear to be most significant in occupational settings. Total phenol exposure for workers exposed to phenol in the workplace is probably substantially higher than for those not exposed in the workplace.

Exposure to benzene is likely to increase phenol exposure because phenol is a metabolite of benzene and is often used to detect benzene exposure. Major releases of benzene include cigarette smoke, auto exhaust, and gasoline fumes.

\subsection{EXPOSURES OF CHILDREN}

This section focuses on exposures from conception to maturity at 18 years in humans. Differences from adults in susceptibility to hazardous substances are discussed in Section 3.7, Children's Susceptibility.

Children are not small adults. A child's exposure may differ from an adult's exposure in many ways. Children drink more fluids, eat more food, breathe more air per kilogram of body weight, and have a larger skin surface in proportion to their body volume. A child's diet often differs from that of adults. The developing human's source of nutrition changes with age: from placental nourishment to breast milk or formula to the diet of older children who eat more of certain types of foods than adults. A child's behavior and lifestyle also influence exposure. Children crawl on the floor, put things in their mouths, sometimes eat inappropriate things (such as dirt or paint chips), and spend more time outdoors. Children also are closer to the ground, and they do not use the judgment of adults to avoid hazards (NRC 1993).

Oral, dermal, and combined oral-dermal exposures are the most likely routes by which children will be exposed to phenol. Oral exposure to low levels of phenol among children is likely because many consumer products contain phenol, particularly in medicines such as gargles, throat lozenges, and others (Darisimall 2006). Products other than medicines that contain phenols include general disinfectants, cleaners, and epoxies. 
Some foods containing phenol have been identified (see Section 6.4.4) and ingestion of these could result in low levels of phenol exposure in children. In addition, phenol is produced endogenously as a breakdown product of protein metabolism; normal concentrations in urine generally do not exceed $20 \mathrm{mg} / \mathrm{L}$ (ACGIH 2001).

Since phenol can be readily absorbed through the skin (ACGIH 2001), children may be more susceptible to low levels of phenol exposure since they have a higher skin-surface-area to weight ratio. Since young children are more likely to come in contact with the floor and other low-lying areas, they may be exposed to phenol found in consumer products such as general disinfectants used to clean toilets, floors, drains, and other areas (Lewis 2001; O’Neil 2001).

Exposure to phenol through inhalation is a less probable route than oral and dermal. It is known that both cigarettes and cigars contain small amounts (19-140 $\mu \mathrm{g}$ ) of phenol (IARC 1986; NCI 1998), and smoking these products indoors produces a measurable amount of phenol (Guerin et al. 1992). If children are present in indoor environments polluted with tobacco smoke, they may be exposed to low levels of phenol.

\subsection{POPULATIONS WITH POTENTIALLY HIGH EXPOSURES}

Populations with potentially high exposure to phenol generally include those who are exposed to relatively highly contaminated environments over long periods of time. These include populations exposed to both identified and unidentified phenol-containing waste disposal sites and landfills. Populations residing in the vicinity of industries that manufacture or use phenol and large population centers may be exposed to potentially high levels of phenol. Persons who work at establishments that manufacture or use phenol have a risk for high exposure to phenol. Populations that regularly ingest food contaminated with phenol or that regularly ingest or come in contact with phenol-containing products are at risk for high exposure to phenol. Populations that live near a phenol spill site, especially those whose water supply sources are near the spill sites, have a risk for high exposure to phenol. Relatively high exposure may also result from exposure to emissions from municipal waste incinerators and cigarette smoke, although no quantitative data concerning phenol emission from these sources were located. Low income communities and minority populations are more likely to live adjacent to waste disposal sites and landfills where phenol is present. 
THE NOES conducted by NIOSH estimated that 584,372 workers were exposed to phenol in the United States (CDC Website). Workers in the petroleum industry, as well as workers in industries that manufacture of caprolactam (nylon manufacture), bisphenol A (epoxy resin and polycarbonate manufacture), herbicides, wood preservatives, hydraulic fluids, heavy-duty surfactants, lube-oil additives, tank linings and coatings, and intermediates for plasticizers and other specialty chemicals, may be exposed to phenol. Phenol is also used in throat lozenges, disinfectants, and ointments. It is also used for facial skin peels and to cause nerve block (Gingell et al. 2001). A list of estimated work induced exposure events for 19 different industries is presented in Table 6-2 (Brandorff et al. 1995).

People who are exposed to large amounts of benzene are also likely to be exposed to large amounts of phenol, a metabolite of benzene. Elevated levels of phenol have been detected in workers occupationally exposed to benzene. Workers exposed to $0.8-25.1$ or $33.1-331.7 \mathrm{ppm}$ for $2.5-3$ hours during an 8 -hour workday had median urinary phenol levels of 55.6 and $351 \mathrm{ng} / \mathrm{g}$ creatine, respectively (Rothman et al. 1998). Control workers who were not exposed to benzene at the workplace had urinary phenol levels of $17.3 \mathrm{ng} / \mathrm{g}$ creatine (Rothman et al. 1998).

\subsection{ADEQUACY OF THE DATABASE}

Section 104(i)(5) of CERCLA, as amended, directs the Administrator of ATSDR (in consultation with the Administrator of EPA and agencies and programs of the Public Health Service) to assess whether adequate information on the health effects of phenol is available. Where adequate information is not available, ATSDR, in conjunction with NTP, is required to assure the initiation of a program of research designed to determine the health effects (and techniques for developing methods to determine such health effects) of phenol.

\subsubsection{Identification of Data Needs}

Physical and Chemical Properties. Knowledge of physical and chemical properties is essential for estimating the partitioning of a chemical in the environment. Information about the physical and chemical properties of phenol is available (Hawley 1981; HSDB 2008; IARC 1989), and the database is adequate for the input requirements of environmental models that predict the behavior of a chemical under specific conditions.

Production, Import/Export, Use, Release, and Disposal. According to the Emergency Planning and Community Right-to-Know Act of 1986, 42 U.S.C. Section 11023, industries are required 


\section{Table 6-2. Estimated Exposure To Phenol At Various Industry Sites}

\begin{tabular}{lcc}
\hline Industry & $\begin{array}{c}\text { Estimated number of } \\
\text { exposure events }\end{array}$ & $\begin{array}{c}\text { Estimated weight used in } \\
\text { tonnes of substance }\end{array}$ \\
\hline Manufacture of basic metals & 180 & 1.7 \\
Manufacture of fabricated metal products & 8,200 & 27 \\
Electrical machinery and apparatus & 1,400 & 0.0012 \\
Manufacture of transport equipment & 700 & 40 \\
Painters and carpenters & 440 & 0.021 \\
Construction & 4,300 & 11 \\
Wholesale trade & 840 & 16 \\
Manufacture of textiles and leather & 2,600 & 1 \\
Manufacture of wood and furniture & 670 & 1.2 \\
Manufacture of chemicals & 580 & 0.85 \\
Manufacture of non-metallic mineral & 1,300 & 4,100 \\
products & 1,300 & 0.0023 \\
Manufacture of precision and optical & & \\
instruments & 840 & $<0.0001$ \\
Manufacture of plastic and boat building & & 0.0002 \\
repair & 260 & 0.0009 \\
Personal services, cleaning, and hair & & 0.65 \\
dressing & 210 & 0.25 \\
Sewage and refuse disposal & 550 & \\
Agricultural, hunting, and forestry & 1,200 & \\
Health sciences and pharmacies & &
\end{tabular}

Source: Brandorff et al. 1995 
to submit substance release and off-site transfer information to the EPA. The TRI, which contains this information for 2005, became available in May of 2007. This database is updated yearly and should provide a list of industrial production facilities and emissions.

Additional production data are available from the Chemical Marketing Reporter (CMR 2005), and import/export data for phenol are available on the U.S. International Trade Commission website (USITC 2008). Additional data are not needed at this time.

Environmental Fate. Based on the physical properties of phenol, volatilization and sorption of phenol to sediments are not expected to be important transport mechanisms (Lyman et al. 1982). The adsorption of phenol to soils has been shown to increase with increasing organic matter (Xing et al. 1994). Photochemical degradation of phenol is thought to be an important process both in air (EPA 1979a) and water (Scully and Hoigne 1987). Phenol is also readily biodegradable (Ludzack and Ettinger 1960; Rubin and Alexander 1983; Scott et al. 1983; Stover and Kincannon 1983). Soil sorption data are available (Artiola-Fortuny and Fuller 1982; Boyd 1982; Briggs 1981; Sacan and Balcioglu 1996; Scott et al. 1983). Additional data are not needed at this time.

Bioavailability from Environmental Media. Data from monitoring studies indicate that phenol is present in the environment (EPA 1981a, 1982; Gossett et al. 1983; Hoff and Chan 1987; Konasewich et al. 1978; Sheldon and Hites 1978, 1979; Thurman 1985) as well as in environmental organisms (Nicola et al. 1987). Exposure to phenol is most likely to be highest in areas at or near industrial centers and population centers where drinking and bathing water, ambient air, and certain foods, such as fish, are obtained from sources contaminated with phenol. Reliable data on the bioavailability of phenol from inhaled air and from skin exposed to phenol vapor have been reported for humans (Piotrowski 1971). Studies of bioavailability of phenol from ingested soil and foods and dermal contact with contaminated water are needed for evaluating the hazards posed by ingesting materials that have been contaminated with phenol.

Food Chain Bioaccumulation. No studies were located regarding the food chain bioaccumulation of phenol from environmental media. Data from monitoring studies indicate that phenol is present in the environment as well as in environmental organisms (Nicola et al. 1987). The available bioaccumulation studies are concerned only with exposure of fish to aqueous concentrations of phenol. Although the results of these studies indicate a low potential for bioaccumulation (see Section 6.3.1), the detection of phenol in fish (see Section 6.4.4) indicates that phenol can be found in aquatic organisms; it is possible 
that food chain bioaccumulation may occur. A clearer understanding of the potential for bioaccumulation would aid in determining how levels in the environment affect the food chain and potentially impact human exposure levels. A study examining phenol levels in organisms from several trophic levels is needed.

Exposure Levels in Environmental Media. Reliable monitoring data for the levels of phenol in contaminated media at hazardous waste sites are needed so that the information obtained on levels of phenol in the environment can be used in combination with the known body burden of phenol to assess the potential risk of adverse health effects in populations living in the vicinity of hazardous waste sites.

Phenol has been measured in air (Cronn et al. 1977; EPA 1981a, 1982), water (EPA 1980; Sheldon and Hites 1978, 1979; Thurman 1985), and sediments (Gossett et al. 1983). Additional more recent monitoring data would help to estimate human exposure to phenol.

Exposure Levels in Humans. Data concerning exposure levels in humans are incomplete and not current (Heikkila et al. 1987; Kuwata et al. 1980; Ohtsuji and Ikeda 1972). A detailed recent database of exposure would be helpful in determining the current exposure levels, thereby allowing the estimation of the average daily dose associated with various scenarios such as living near a hazardous waste site or landfill, or with drinking water containing phenol. An environmental media monitoring program would provide the necessary information for estimating environmental exposures, while a detailed examination of the uses of phenol and the kinds of potential exposure in addition to workplace monitoring would probably provide adequate workplace information. The environmental media that would provide the most useful information are air, groundwater, and surface and drinking water in urban and industrial locations, and air, groundwater, and surface water at hazardous waste sites. Performing the monitoring over a 1 -year period would allow estimation of seasonal variations.

This information is necessary for assessing the need to conduct health studies on these populations.

Exposures of Children. Children are likely to be exposed to low levels of phenol from the use of many consumer products including medicines and cleaning agents (Budavari et al. 1989; Douglas 1972; EPA 1980; Hawley 1981). There are no known data that quantify the level of exposure to phenol in children. It is likely that young children may be exposed to low levels of phenol because they come into contact with the floor and other areas where disinfectants containing phenol might be used. More studies are needed to assess whether children differ in their weight-adjusted intake of phenol, as little or no 
information is known. Studies are needed to measure the baseline phenol level in children's urine in order to use phenol levels in urine as a biomarker of exposure.

Child health data needs relating to susceptibility are discussed in Section 3.12.2, Identification of Data Needs: Children's Susceptibility.

Exposure Registries. No exposure registries for phenol were located. This substance is not currently one of the compounds for which a sub-registry has been established in the National Exposure Registry. The substance will be considered in the future when chemical selection is made for subregistries to be established. The information that is amassed in the National Exposure Registry facilitates the epidemiological research needed to assess adverse health outcomes that may be related to exposure to this substance.

\subsubsection{Ongoing Studies}

The Federal Research in Progress (FEDRIP 2006) database provides additional information obtainable from a few ongoing studies that may fill in some of the data needs identified in Section 6.8.1. The only study pertaining to the potential for human exposure found in this database was an exposure assessment study done at Mount Sinai School of Medicine at New York University and supported by National Institute of Environmental Health Sciences. Part of this research is to design new approaches to assess and quantify the levels of exposures to known toxicants in children and infants. 


\section{ANALYTICAL METHODS}

The purpose of this chapter is to describe the analytical methods that are available for detecting, measuring, and/or monitoring phenol, its metabolites, and other biomarkers of exposure and effect to phenol. The intent is not to provide an exhaustive list of analytical methods. Rather, the intention is to identify well-established methods that are used as the standard methods of analysis. Many of the analytical methods used for environmental samples are the methods approved by federal agencies and organizations such as EPA and the National Institute for Occupational Safety and Health (NIOSH). Other methods presented in this chapter are those that are approved by groups such as the Association of Official Analytical Chemists (AOAC) and the American Public Health Association (APHA). Additionally, analytical methods are included that modify previously used methods to obtain lower detection limits and/or to improve accuracy and precision.

\subsection{BIOLOGICAL MATERIALS}

Analytical methods for the detection of phenol in biological materials are summarized in Table 7-1. Phenol is expected to be present in blood and urine in its free acid and conjugated forms (glucuronide and sulfate). The average urinary phenol concentration in unexposed individuals is $9.5 \pm 3.6 \mathrm{mg} / \mathrm{L}$ when corrected to a standard specific gravity of 1.024 (Piotrowski 1971). In exposed individuals, the urinary phenol level may vary from 10 to $200 \mathrm{mg} / \mathrm{L}$ (Tesarova and Packova 1983). The two common methods for quantifying conjugated phenol are chemical and enzymatic hydrolysis of the conjugate to the free phenol form. The chemical method uses acidic hydrolysis (Baldwin et al. 1981; Needham et al. 1984). Both the nature of the acid (sulfuric versus perchloric) and the temperature should be controlled carefully to obtain a quantitative yield and to avoid thermal decomposition of other phenolic or related compounds that may interfere with phenol quantification (Baldwin et al. 1981; Rick et al. 1982). The best available method appears to be specific enzyme hydrolysis or hydrolysis at ambient temperature with sulfuric acid. Enzymatic hydrolysis with an extract of Helix pomatia has also been used to liberate phenol from its conjugates (Ahmed and Hale 1994).

High-performance liquid chromatographic separation with electrochemical detection may provide the best sensitivity for phenol quantification in biological samples (Tesarova and Packova 1983). The use of gas chromatography with a flame ionization detector may be a more versatile method, if other non-ionic pollutants must be quantified. The advantages and disadvantages of different methods available for the quantification of phenol and metabolites in biological and environmental samples have been discussed by Tesarova and Packova (1983). 


\section{Table 7-1. Analytical Methods for Determining Phenol in Biological Samples}

\begin{tabular}{|c|c|c|c|c|c|}
\hline Sample matri & Preparation method & $\begin{array}{l}\text { Analytical } \\
\text { method }\end{array}$ & $\begin{array}{l}\text { Sample } \\
\text { detection } \\
\text { limit }\end{array}$ & $\begin{array}{l}\text { Percent } \\
\text { recovery }\end{array}$ & Reference \\
\hline Whole blood & $\begin{array}{l}\text { Sample extracted with ethyl } \\
\text { acetate, extract concentrated } \\
\text { and analyzed (for free phenol), } \\
\text { packed blood cells previously } \\
\text { extracted for free phenol } \\
\text { incubated with } \beta \text {-glucuronidase } \\
\text { containing sulfatase at } 37^{\circ} \mathrm{C} \text {, } \\
\text { extracted with ethyl acetate } \\
\text { after addition of normal saline } \\
\text { solution, and extract } \\
\text { concentrated and analyzed (for } \\
\text { conjugated phenol) }\end{array}$ & GC-FID & $<1 \mathrm{mg} / \mathrm{mL}$ & $\begin{array}{l}97 \% \text { (free } \\
\text { phenol); } 103 \% \\
\text { (conjugated } \\
\text { phenol) }\end{array}$ & $\begin{array}{l}\text { O'Grodnick et } \\
\text { al. } 1983\end{array}$ \\
\hline Whole blood & $\begin{array}{l}\text { Sample with spiked internal } \\
\text { standard extracted with ethyl } \\
\text { acetate and extract } \\
\text { concentrated and analyzed }\end{array}$ & GC-FID & $0.1 \mathrm{mg} / \mathrm{L}$ & $\begin{array}{l}>90 \% \text { at } \\
\text { concentrations } \\
\text { above } 0.5 \mathrm{mg} / \mathrm{L}\end{array}$ & $\begin{array}{l}\text { Handson and } \\
\text { Hanrahan } 1983\end{array}$ \\
\hline Urine & $\begin{array}{l}\text { Sample mixed with phosphoric } \\
\text { acid, passed through a pre- } \\
\text { column at } 165^{\circ} \mathrm{C} \text { for hydrolysis } \\
\text { of conjugates (for free and } \\
\text { conjugated phenol), and } \\
\text { analyzed }\end{array}$ & GC-FID & NG & $\begin{array}{l}89 \% \text { (for } \\
\text { conjugates) }\end{array}$ & $\begin{array}{l}\text { Baldwin et al. } \\
1981\end{array}$ \\
\hline Urine & $\begin{array}{l}\text { Sample heated under reflux } \\
\text { with } \mathrm{HClO}_{4} \text {, solvent extracted, } \\
\text { concentrated, and separated } \\
\text { by TLC; spot developed by } \\
\text { p-nitro-benzenediazonium } \\
\text { fluoroborate, removed } \\
\text { quantitatively and solvent } \\
\text { extracted (for free and } \\
\text { conjugated phenol), and } \\
\text { analyzed }\end{array}$ & $\begin{array}{l}\text { Spectro- } \\
\text { photometry }\end{array}$ & NG & NG & $\begin{array}{l}\text { Bienick and } \\
\text { Wilczok } 1986\end{array}$ \\
\hline Urine & $\begin{array}{l}\text { Acidified sample stream } \\
\text { distilled, reacted with ammonia, } \\
N \text {-chloro-succinimide, and } \\
\text { sodium nitroprusside at basic } \\
\text { pH (method probably for free } \\
\text { phenol), and analyzed }\end{array}$ & $\begin{array}{l}\text { Spectro- } \\
\text { photometry }\end{array}$ & $0.3 \mathrm{mg} / \mathrm{L}$ & $>95 \%$ & $\begin{array}{l}\text { Amlathe et al. } \\
1987\end{array}$ \\
\hline Urine & $\begin{array}{l}\text { Sample incubated with } \\
\text { glucuronidase and sulfatase at } \\
\text { pH } 5 \text { and } 3.7^{\circ} \mathrm{C}, \mathrm{H}_{2} \mathrm{SO}_{4} \text { added } \\
\text { and steam distilled (total } \\
\text { phenol) and analyzed }\end{array}$ & $\begin{array}{l}\text { HPLC- } \\
\text { electro- } \\
\text { chemical } \\
\text { detector }\end{array}$ & $\begin{array}{l}2 \mathrm{ng} / \\
\text { inection }\end{array}$ & 95-107\% & $\begin{array}{l}\text { Schaltenbrand } \\
\text { and Coburn } \\
1985\end{array}$ \\
\hline Urine & $\begin{array}{l}\text { Sample hydrolyzed at room } \\
\text { temperature and extracted with } \\
\text { methyl tert-butyl ether (total } \\
\text { phenol) and analyzed }\end{array}$ & GC-FID & NG & NG & Rick et al. 1982 \\
\hline
\end{tabular}




\section{Table 7-1. Analytical Methods for Determining Phenol in Biological Samples}

\begin{tabular}{|c|c|c|c|c|c|}
\hline Sample matrix & Preparation method & $\begin{array}{l}\text { Analytical } \\
\text { method }\end{array}$ & $\begin{array}{l}\text { Sample } \\
\text { detection } \\
\text { limit }\end{array}$ & $\begin{array}{l}\text { Percent } \\
\text { recovery }\end{array}$ & Reference \\
\hline Urine & $\begin{array}{l}\text { Sample spiked with internal } \\
\text { standard, hydrolyzed with } \\
\mathrm{H}_{2} \mathrm{SO}_{4} \text {, and extracted with ethyl } \\
\text { acetate (free and conjugated) } \\
\text { and analyzed }\end{array}$ & GC-FID & NG & $\begin{array}{l}93-97 \% \text { at } 20- \\
70 \mathrm{mg} / \mathrm{L}\end{array}$ & $\begin{array}{l}\text { Needham et al. } \\
1984\end{array}$ \\
\hline Urine & $\begin{array}{l}\text { Sample spiked with internal } \\
\text { standard, distilled with } \mathrm{H}_{2} \mathrm{SO}_{4} \\
\text { in a special apparatus, distillate } \\
\text { directly injected into } \mathrm{GC} \text { (free } \\
\text { and conjugated) }\end{array}$ & GC-FID & $0.1 \mathrm{mg} / \mathrm{L}$ & $\begin{array}{l}99 \% \text { at } \\
5.9 \mathrm{mg} / \mathrm{L}\end{array}$ & $\begin{array}{l}\text { Van } \\
\text { Roosmalen et } \\
\text { al. } 1981\end{array}$ \\
\hline Urine & $\begin{array}{l}\text { Two spot urine samples } \\
\text { (before and after exposure), } \\
\text { hydrolyzed with HCl or } \\
\text { perchloric acid, extracted with } \\
\text { diethyl ether, and directly } \\
\text { injected into GC }\end{array}$ & $\begin{array}{l}\text { GC-FID } \\
\text { (Method } \\
\text { 8305) }\end{array}$ & $0.5 \mu \mathrm{g} / \mathrm{mL}$ & $94-95 \%$ & NIOSH 1994a \\
\hline
\end{tabular}

$\mathrm{FID}=$ flame ionization detector; $\mathrm{GC}=$ gas chromatography; $\mathrm{H}_{2} \mathrm{SO}_{4}=$ sulfuric acid; $\mathrm{HCl}=$ hydrochloric acid; $\mathrm{HClO}_{4}=$ perchloric acid; HPLC = high performance liquid chromatography; NG = not given; TLC = thin layer chromatography 
The level of phenol detected in blood or urine may not accurately reflect actual phenol exposure because phenol may also appear as a metabolite of benzene or other drugs. It has been shown that under certain acidic conditions used for the hydrolysis of conjugated phenols, acetyl salicylic acid (aspirin) may produce phenol (Baldwin et al. 1981) and yield spuriously higher values for phenol in blood and urine.

For occupational exposure, it is recommended that urine samples be collected at the end of an 8-hour work shift (ACGIH 2001). Small amounts of thymol can be used as a preservative, and the urine can be stored for 4 days if refrigerated, or at least 3 months if frozen.

\subsection{ENVIRONMENTAL SAMPLES}

Analytical methods for detecting phenol in environmental samples are summarized in Table 7-2. The accuracy and sensitivity of phenol determination in environmental samples depends on sample preconcentration and pretreatment and the analytical method employed. The recovery of phenol from air and water by the various preconcentration methods is usually low for samples containing low levels of phenol. The two preconcentration methods commonly used for phenols in water are adsorption on XAD resin and adsorption on carbon. Both can give low recoveries, as shown by Van Rossum and Webb (1978). Solvent extraction at acidic $\mathrm{pH}$ with subsequent solvent concentration also gives unsatisfactory recovery for phenol. Even during carefully controlled conditions, phenol losses of up to $60 \%$ may occur during solvent evaporation (Handson and Hanrahan 1983). The in situ acetylation with subsequent solvent extraction as developed by Sithole et al. (1986) is probably one of the most promising methods.

Capillary columns may provide the best method for the separation of phenols prior to their quantification (Eichelberger et al. 1983; Shafer et al. 1981; Sithole et al. 1986). Of the various methods available for detection, the two commonly used methods that are most sensitive are mass spectrometry and flame ionization detection. Although electron capture detectors provide good sensitivities for higher chlorinesubstituted phenols, they are poor for phenol itself (Sithole et al. 1986). The best method for the quantification of phenol may be mass spectrometric detection in the selected ion mode, but the loss of qualitative information may be significant (Eichelberger et al. 1983).

\subsection{ADEQUACY OF THE DATABASE}

Section 104(i)(5) of CERCLA, as amended, directs the Administrator of ATSDR (in consultation with the Administrator of EPA and agencies and programs of the Public Health Service) to assess whether adequate information on the health effects of phenol is available. Where adequate information is not 
Table 7-2. Analytical Methods for Determining Phenol in Environmental Samples

\begin{tabular}{|c|c|c|c|c|c|}
\hline $\begin{array}{l}\text { Sample } \\
\text { matrix }\end{array}$ & Preparation method & $\begin{array}{l}\text { Analytical } \\
\text { method }\end{array}$ & $\begin{array}{l}\text { Sample } \\
\text { detection limit }\end{array}$ & $\begin{array}{l}\text { Percent } \\
\text { recovery }\end{array}$ & Reference \\
\hline Urban air & $\begin{array}{l}\text { Sample collected in bubbler } \\
\text { containing } \mathrm{NaOH} \text {, derivatized } \\
\text { as nitrobenzeneazo compound }\end{array}$ & HPLC-UV & $\begin{array}{l}0.05 \mathrm{ppb} \text { for } \\
150-\mathrm{L} \text { sample; } \\
58-60 \% \text { at } \\
0.33-0.5 \mu \mathrm{g} \\
\text { phenol }\end{array}$ & $\begin{array}{l}72.3 \% \text { at } \\
10-50 \mu \mathrm{g} \\
\text { phenol }\end{array}$ & $\begin{array}{l}\text { Kuwata et al. } \\
1980\end{array}$ \\
\hline Air & $\begin{array}{l}\text { Sample collected on a solid } \\
\text { sorbent tube, desorbed using } \\
\text { methanol }\end{array}$ & $\begin{array}{l}\text { GC-FID } \\
\text { (Method } \\
\text { 2546) }\end{array}$ & $1-3 \mu \mathrm{g} / \mathrm{sample}$ & ND & NIOSH 1994b \\
\hline $\begin{array}{l}\text { Occupational } \\
\text { air }\end{array}$ & $\begin{array}{l}\text { Sorption on activated carbon, } \\
\text { desorption by solvent and } \\
\text { derivatized to trimethylsilyl } \\
\text { product }\end{array}$ & GC-FID & $\begin{array}{l}0.5 \mathrm{mg} / \mathrm{m}^{3} \\
(0.13 \mathrm{ppm})\end{array}$ & $\begin{array}{l}96-102 \% \\
\text { at } 2.5- \\
100 \mathrm{mg} / \mathrm{m}^{3}\end{array}$ & $\begin{array}{l}\text { Yrjanheikki } \\
1987\end{array}$ \\
\hline $\begin{array}{l}\text { Occupational } \\
\text { air }\end{array}$ & $\begin{array}{l}\text { Sorption on XAD-2, desorption } \\
\text { by acetonitrile and } \\
\text { concentrated if necessary }\end{array}$ & $\begin{array}{l}\text { HPLC- } \\
\text { electro- } \\
\text { chemical } \\
\text { detector and } \\
\text { HPLC-UV }\end{array}$ & $\begin{array}{l}8 \mu \mathrm{g} / \mathrm{m}^{3} \\
(2.04 \mathrm{ppb}) \text { with } \\
12 \mathrm{~L} \text { air } \\
\text { (electro- } \\
\text { chemical); } \\
0.16 \mathrm{mg} / \mathrm{m}^{3} \\
(0.04 \mathrm{ppm}) \text { with } \\
12 \mathrm{~L} \text { air }(\mathrm{UV})\end{array}$ & ND & $\begin{array}{l}\text { Nieminen and } \\
\text { Heikkila } 1986\end{array}$ \\
\hline $\begin{array}{l}\text { Occupational } \\
\text { air }\end{array}$ & $\begin{array}{l}\text { Sample collected with a } \\
\text { thermal desorption tube using a } \\
\text { sorbent capable of capturing a } \\
\mathrm{C}_{6} \text { organic compound }\end{array}$ & $\begin{array}{l}\text { GC-MS } \\
\text { (Method } \\
\text { 2549) }\end{array}$ & $\begin{array}{l}100 \mathrm{ng} / \mathrm{tube} \text { or } \\
\text { less }\end{array}$ & NA & NIOSH 1996 \\
\hline $\begin{array}{l}\text { Total } \\
\text { particulate } \\
\text { matter in } \\
\text { cigarette } \\
\text { smoke }\end{array}$ & $\begin{array}{l}\text { Extract particulate matter with } \\
\mathrm{NaOH}, \text { buffer to } \mathrm{pH} 4.6\end{array}$ & $\begin{array}{l}\text { HPLC- } \\
\text { fluorescence } \\
\text { spectrophoto- } \\
\text { meter }\end{array}$ & $0.3 \mathrm{mg} / \mathrm{L}$ & $\begin{array}{l}91 \% \text { at } 20- \\
30 \mu g\end{array}$ & $\begin{array}{l}\text { Tomkins et al. } \\
1984\end{array}$ \\
\hline $\begin{array}{l}\text { Industrial } \\
\text { emission, } \\
\text { auto exhaust, } \\
\text { and tobacco } \\
\text { smoke }\end{array}$ & $\begin{array}{l}\text { Sample collected in } \mathrm{NaOH} \\
\text { bubbler and derivatized to } \\
p \text {-nitrobenzene-diazonium } \\
\text { tetrafluoroborate }\end{array}$ & HPLC-UV & $\begin{array}{l}0.05 \mathrm{ppb} \text { for } \\
150-\mathrm{L} \text { sample }\end{array}$ & ND & $\begin{array}{l}\text { Kuwata et al. } \\
1980\end{array}$ \\
\hline $\begin{array}{l}\text { Drinking } \\
\text { water, waste } \\
\text { water, and } \\
\text { natural water }\end{array}$ & $\begin{array}{l}\text { Direct distillation of solvent- } \\
\text { cleaned sample (if necessary) } \\
\text { at acidic } \mathrm{pH} \text {, react with } \\
\text { 4-amino-antipyrine and } \\
\text { potassium ferricyanide at } \mathrm{pH} \text { 8, } \\
\text { extract in chloroform }\end{array}$ & $\begin{array}{l}\text { Spectrophoto- } \\
\text { metric } \\
\text { (Method } \\
5530 \mathrm{c} \text { ) }\end{array}$ & $\begin{array}{l}1 \mu \mathrm{g} / \mathrm{L} \text { for } \\
500-\mathrm{mL} \text { sample }\end{array}$ & ND & APHA 1998a \\
\hline $\begin{array}{l}\text { Waste water } \\
\text { and natural } \\
\text { water }\end{array}$ & None & $\begin{array}{l}\text { GC-FID } \\
\text { (Method } \\
6420 b \text { ) }\end{array}$ & $<1 \mathrm{mg} / \mathrm{L}$ & ND & APHA 1998b \\
\hline Water & $\begin{array}{l}\text { 1-L sample acidified and } \\
\text { extracted with methylene } \\
\text { chloride }\end{array}$ & $\begin{array}{l}\text { GC-FID } \\
\text { (Method 604) }\end{array}$ & $0.14 \mu \mathrm{g} / \mathrm{L}$ & $44 \%$ & EPA 2001a \\
\hline
\end{tabular}


Table 7-2. Analytical Methods for Determining Phenol in Environmental Samples

\begin{tabular}{|c|c|c|c|c|c|}
\hline $\begin{array}{l}\text { Sample } \\
\text { matrix }\end{array}$ & Preparation method & $\begin{array}{l}\text { Analytical } \\
\text { method }\end{array}$ & $\begin{array}{l}\text { Sample } \\
\text { detection limit }\end{array}$ & $\begin{array}{l}\text { Percent } \\
\text { recovery }\end{array}$ & Reference \\
\hline Water & $\begin{array}{l}\text { 1-L sample is adjusted to } \mathrm{pH} \\
>11 \text { and extracted with } \\
\text { methylene chloride }\end{array}$ & $\begin{array}{l}\text { GC-MS } \\
\text { (Method 635) }\end{array}$ & $1.5 \mu \mathrm{g} / \mathrm{L}$ & $56 \%$ & EPA 2001c \\
\hline $\begin{array}{l}\text { Water, waste } \\
\text { water }\end{array}$ & $\begin{array}{l}\text { Acidified sample extract with } \\
\text { solvent, concentrated or } \\
\text { derivatized to pentafluoro- } \\
\text { benzylbromide product }\end{array}$ & $\begin{array}{l}\text { GC-FID; GC- } \\
\text { ECD (for } \\
\text { derivatized } \\
\text { EPA Method } \\
604 \text { ) }\end{array}$ & $\begin{array}{l}0.14 \mu \mathrm{g} / \mathrm{L} \\
(\text { FID); } 2.2 \mu \mathrm{g} / \mathrm{L} \\
\text { (ECD) }\end{array}$ & $\begin{array}{l}41 \%(F I D) \\
\text { ND (ECD) }\end{array}$ & EPA 1982 \\
\hline $\begin{array}{l}\text { Water, waste } \\
\text { water }\end{array}$ & $\begin{array}{l}\text { Sample extracted in acidic } \mathrm{pH} \text {, } \\
\text { extract concentrated }\end{array}$ & $\begin{array}{l}\text { GC-MS (EPA } \\
\text { Method 625); } \\
\text { HRGC-MS } \\
\text { (EPA Method } \\
625.1 \text { ) }\end{array}$ & $\begin{array}{l}1.5 \mu \mathrm{g} / \mathrm{L}(\mathrm{GC}- \\
\mathrm{MS}) ; 1-10 \mu \mathrm{g} / \mathrm{L} \\
\text { (HRGC-MS) }\end{array}$ & $\begin{array}{l}36 \%(\mathrm{GC}- \\
\mathrm{MS}) \text { at } 10- \\
1,500 \mu \mathrm{g} / \mathrm{L} ; \\
25 \%(\mathrm{GC}- \\
\mathrm{MS}) \text { at } \\
8.3 \mu \mathrm{g} / \mathrm{L} ; \\
42 \% \\
\text { (HRGC- } \\
\text { MS) at } \\
20 \mu \mathrm{g} / \mathrm{L}\end{array}$ & $\begin{array}{l}\text { Eichelberger } \\
\text { et al. 1983; } \\
\text { EPA } 1982\end{array}$ \\
\hline Water & $\begin{array}{l}\text { Sample passed through } \\
\text { graphitized carbon black, } \\
\text { eluted with methylene chloride }\end{array}$ & $\begin{array}{l}\text { Ion- } \\
\text { suppression; } \\
\text { reversed } \\
\text { phase LC } \\
\text { with UV } \\
\text { detection }\end{array}$ & 50-100 ng/L & $91-97 \%$ & $\begin{array}{l}\text { Di Corcia et } \\
\text { al. } 1996\end{array}$ \\
\hline Water & $\begin{array}{l}\text { Sample passed through a } \\
\text { mixed XAD- } 4 / 8 \text { column, solvent } \\
\text { eluted and concentrated }\end{array}$ & GC-MS & ND & $\begin{array}{l}46-70 \% \\
\text { (distilled } \\
\text { water); } 9 \% \\
\text { (tap water) }\end{array}$ & $\begin{array}{l}\text { Van Rossum } \\
\text { and Webb } \\
1978\end{array}$ \\
\hline Waste water & $\begin{array}{l}\text { Distillation of acidified solution, } \\
\text { reacted with ammonia, } \\
\mathrm{N} \text {-chloro-succinimide, and } \\
\text { sodium nitro-prusside at basic } \\
\text { pH }\end{array}$ & $\begin{array}{l}\text { Spectrophoto- } \\
\text { metric }\end{array}$ & $<0.3 \mathrm{mg} / \mathrm{L}$ & $\begin{array}{l}96.7 \% \text { at } \\
3 \mathrm{mg} / \mathrm{L}\end{array}$ & $\begin{array}{l}\text { Amlathe et al. } \\
1987\end{array}$ \\
\hline $\begin{array}{l}\text { Potable water } \\
\text { and raw } \\
\text { source water }\end{array}$ & $\begin{array}{l}\text { Sample acetylated in situ by } \\
\text { addition of acetic anhydride, } \\
\text { solvent extracted and } \\
\text { concentrated; alternatively, } \\
\text { extracted acidic sample } \\
\text { derivatized by } \\
\text { pentafluorobenzyl bromide and } \\
\text { cleaned up by column } \\
\text { chromatography }\end{array}$ & $\begin{array}{l}\text { HRGC-ECD } \\
\text { (for } \\
\text { pentafluoro- } \\
\text { benzyl } \\
\text { derivative); } \\
\text { HRGC-MS } \\
\text { (for acetyl } \\
\text { derivative) }\end{array}$ & $\begin{array}{l}<50 \mathrm{ng} / \mathrm{L} \\
\text { (pentafluoro- } \\
\text { benzyl); } \\
<50 \mathrm{ng} / \mathrm{L} \\
\text { (acetyl } \\
\text { derivative) }\end{array}$ & $\begin{array}{l}\text { 10-64\% } \\
\text { (penta- } \\
\text { fluoro- } \\
\text { benzyl } \\
\text { derivative); } \\
70-132 \% \\
\text { (acetyl } \\
\text { derivative) }\end{array}$ & $\begin{array}{l}\text { Sithole et al. } \\
1986\end{array}$ \\
\hline $\begin{array}{l}\text { Drinking } \\
\text { water }\end{array}$ & $\begin{array}{l}\text { 1-L sample is extracted using a } \\
\text { solid phase extraction cartridge }\end{array}$ & $\begin{array}{l}\text { GC-MS } \\
\text { (Method 528) }\end{array}$ & $0.026 \mu \mathrm{g} / \mathrm{L}$ & 85 & EPA 2000a \\
\hline
\end{tabular}


Table 7-2. Analytical Methods for Determining Phenol in Environmental Samples

\begin{tabular}{|c|c|c|c|c|c|}
\hline $\begin{array}{l}\text { Sample } \\
\text { matrix }\end{array}$ & Preparation method & $\begin{array}{l}\text { Analytical } \\
\text { method }\end{array}$ & $\begin{array}{l}\text { Sample } \\
\text { detection limit }\end{array}$ & $\begin{array}{l}\text { Percent } \\
\text { recovery }\end{array}$ & Reference \\
\hline Water & $\begin{array}{l}\text { The sample is extracted at } \mathrm{pH} \\
12-13 \text {, then at } \mathrm{pH}<2 \text { with } \\
\text { methylene chloride using } \\
\text { continuous extraction } \\
\text { techniques; the extract is dried } \\
\text { over sodium sulfate and } \\
\text { concentrated to a volume of } \\
1 \mathrm{~mL}\end{array}$ & $\begin{array}{l}\text { GC-MS } \\
\text { (Method } \\
1625 \text { ) }\end{array}$ & Not applicable & $\begin{array}{l}\text { Not } \\
\text { applicable }\end{array}$ & EPA 2001b \\
\hline $\begin{array}{l}\text { Drinking } \\
\text { water }\end{array}$ & $\begin{array}{l}\text { Water samples collected and } \\
\text { analyzed via GC-MS }\end{array}$ & $\begin{array}{l}\text { GC-MS } \\
\text { (Method } \\
\text { OM100R }\end{array}$ & Not applicable & $\begin{array}{l}\text { Not } \\
\text { applicable }\end{array}$ & DOE 1997 \\
\hline $\begin{array}{l}\text { Aqueous } \\
\text { samples }\end{array}$ & $\begin{array}{l}\text { Samples extracted and cleaned } \\
\text { up (according to sample matrix) } \\
\text { and the solvent appropriately } \\
\text { exchanged; the phenols are } \\
\text { then determined with or without } \\
\text { derivatization }\end{array}$ & $\begin{array}{l}\text { GC-MS } \\
\text { (Method } \\
8041 A\end{array}$ & Not applicable & $93 \%$ & EPA 2000b \\
\hline Sediment & $\begin{array}{l}\text { Homogenized sample solvent } \\
\text { extracted at acidic } \mathrm{pH} \text {, } \\
\text { fractionated by GPC and } \\
\text { fractions concentrated }\end{array}$ & HRGC-MS & ND & $\begin{array}{l}112-128 \% \\
\text { at } 400 \mathrm{ng} / \mathrm{g}\end{array}$ & $\begin{array}{l}\text { Lopez-Avila } \\
\text { et al. } 1983\end{array}$ \\
\hline Groundwater & $\begin{array}{l}\text { Solvent extraction in acidic } \mathrm{pH} \text {, } \\
\text { extract concentrated }\end{array}$ & $\begin{array}{l}\text { GC-MS (EPA- } \\
\text { CLP Method) }\end{array}$ & $10 \mu \mathrm{g} / \mathrm{L}$ & ND & EPA 1987 \\
\hline Soil, sediment & $\begin{array}{l}\text { Sample mixed with anhydrous } \\
\text { powdered } \mathrm{Na}_{2} \mathrm{SO}_{4} \text {, solvent } \\
\text { extracted ultrasonically, extract } \\
\text { subjected to GPC if necessary, } \\
\text { extract concentrated }\end{array}$ & $\begin{array}{l}\text { GC-MS (EPA- } \\
\text { CLP Method }\end{array}$ & $330 \mu \mathrm{g} / \mathrm{kg}$ & ND & EPA 1987 \\
\hline $\begin{array}{l}\text { Water and } \\
\text { waste water }\end{array}$ & $\begin{array}{l}\text { Phenols separated on a Nova- } \\
\text { Pak Phenyl column eluted with } \\
\text { ammonium acetate:acetonitrile }\end{array}$ & LC-ED & $0.5 \mathrm{mg} / \mathrm{L}$ & $91-100 \%$ & $\begin{array}{l}\text { Paterson et } \\
\text { al. } 1996\end{array}$ \\
\hline Groundwater & $\begin{array}{l}\text { Solvent extraction, column } \\
\text { chromatographic cleanup, } \\
\text { concentration of extract }\end{array}$ & $\begin{array}{l}\text { GC-MS (EPA } \\
\text { Method } \\
8250 A)\end{array}$ & $1.5 \mathrm{mg} / \mathrm{L}$ & $\begin{array}{l}0.43 c+ \\
1.26) / c x \\
100 \text { where } \\
c \text { is the } \\
\text { actual } \\
\text { concentra- } \\
\text { tion }\end{array}$ & EPA 1994b \\
\hline $\begin{array}{l}\text { Bottom } \\
\text { sediment }\end{array}$ & $\begin{array}{l}\text { Wet sediment samples dried } \\
\text { and compounds extracted } \\
\text { using dichloromethane }\end{array}$ & $\begin{array}{l}\text { GC-MS } \\
\text { (Method 0- } \\
5130-95 \text { ) }\end{array}$ & $23.5 \mu \mathrm{g} / \mathrm{kg}$ & 84 & USGS 1995 \\
\hline Water & $\begin{array}{l}\text { Water samples filtered using } \\
\text { glass fiber filters; samples } \\
\text { extracted using SPE cartridges }\end{array}$ & $\begin{array}{l}\text { GC-MS } \\
\text { (Method 0- } \\
\text { 1433-01) }\end{array}$ & $0.11 \mu \mathrm{g} / \mathrm{L}$ & 93 & USGS 2002 \\
\hline $\begin{array}{l}\text { Soil, sludge, } \\
\text { or solid waste }\end{array}$ & $\begin{array}{l}\text { Extracted by soxhlet or } \\
\text { sonication, extract subjected to } \\
\text { column chromatographic } \\
\text { cleanup and concentrated }\end{array}$ & $\begin{array}{l}\text { GC-MS (EPA } \\
\text { Method } \\
8250 A)\end{array}$ & $1.5 \mathrm{mg} / \mathrm{kg}$ & ND & EPA 1994b \\
\hline
\end{tabular}




\section{Table 7-2. Analytical Methods for Determining Phenol in Environmental Samples}

\begin{tabular}{|c|c|c|c|c|c|}
\hline $\begin{array}{l}\text { Sample } \\
\text { matrix }\end{array}$ & Preparation method & $\begin{array}{l}\text { Analytical } \\
\text { method }\end{array}$ & $\begin{array}{l}\text { Sample } \\
\text { detection limit }\end{array}$ & $\begin{array}{l}\text { Percent } \\
\text { recovery }\end{array}$ & Reference \\
\hline $\begin{array}{l}\text { Aqueous and } \\
\text { nonaqueous } \\
\text { samples }\end{array}$ & $\begin{array}{l}\text { Samples are extracted; extracts } \\
\text { are cleaned up, as necessary, } \\
\text { and the solvent exchanged to } \\
\text { one that is compatible with the } \\
\text { GC detector to be used }\end{array}$ & $\begin{array}{l}\text { EPA Method } \\
8041 \mathrm{~A}\end{array}$ & ND & $93 \%$ & EPA 2007 \\
\hline $\begin{array}{l}\text { Soil, air, } \\
\text { water, }\end{array}$ & $\begin{array}{l}\text { Soxhlet extraction with } \\
\text { acetone/hexane }\end{array}$ & $\begin{array}{l}\text { GC-MS } \\
\text { (Method } \\
\text { 8270D) }\end{array}$ & $10 \mu \mathrm{g} / \mathrm{L}$ & $46 \%$ & EPA 1998 \\
\hline Honey & $\begin{array}{l}\text { Sample dissolved in water, } \\
\text { steam distilled; distillate } \\
\text { cleaned up by column } \\
\text { chromatography }\end{array}$ & HPLC-UP & $\begin{array}{l}0.1 \mathrm{ppm} \text { (for } \\
10-\mathrm{g} \text { sample }\end{array}$ & $\begin{array}{l}98 \% \text { at } \\
111 \mu g \\
\text { added } \\
\text { phenol }\end{array}$ & Sporns 1981 \\
\hline
\end{tabular}

$\mathrm{C}_{6}=6$ carbon; $\mathrm{ECD}=$ electron capture detector; $\mathrm{ED}=$ electrochemical detection; $\mathrm{FID}=$ flame ionization detector; GC = gas chromatography; GPC = gel permeation chromatography; HPLC = high performance liquid chromatography; HRGC = high resolution gas chromatography; LC = liquid chromatography; $M S=$ mass spectrometry; $\mathrm{Na}_{2} \mathrm{SO}_{4}=$ sodium sulfate; $\mathrm{NaOH}=$ sodium hydroxide; $\mathrm{ND}=$ no data; $\mathrm{SPE}=$ solid-phase extraction; UV = ultraviolet detection 
available, ATSDR, in conjunction with NTP, is required to assure the initiation of a program of research designed to determine the health effects (and techniques for developing methods to determine such health effects) of phenol.

The following categories of possible data needs have been identified by a joint team of scientists from ATSDR, NTP, and EPA. They are defined as substance-specific informational needs that if met would reduce the uncertainties of human health assessment. This definition should not be interpreted to mean that all data needs discussed in this section must be filled. In the future, the identified data needs will be evaluated and prioritized, and a substance-specific research agenda will be proposed.

\subsubsection{Identification of Data Needs}

\section{Methods for Determining Biomarkers of Exposure and Effect.}

Exposure. Measurement of total phenol in urine serves as a biomarker of exposure for persons occupationally exposed to phenol (ACGIH 2001).

Effect. Specific biomarkers used to characterize effects caused by phenol have not been identified. Dark urine has been reported in persons occupationally exposed to phenol (inhalation, dermal) (ACGIH 2001; Merliss 1972) and following oral exposure (Baker et al. 1978; Kim et al. 1994). The dark urine may be a result of an oxidation product of phenol, or hemoglobin and hemoglobin breakdown products. Further research is required to identify the cause of the dark urine and relate it to exposure concentration.

\section{Methods for Determining Parent Compounds and Degradation Products in Environmental}

Media. The analytical methods available (Amlathe et al. 1987; Baldwin et al. 1981; Bieniek and Wilczok 1986; Handson and Hanrahan 1983; Needham et al. 1984; O'Grodnick et al. 1983; Rick et al. 1982; Schaltenbrand and Coburn 1985; Van Roosmalen et al. 1981) are adequate for the quantification of phenol and its conjugates in biological samples. The study of the levels of parent compound in human blood, urine, or other biological matrices can be useful in deriving a correlation between the levels of this compound found in the environment and those found in human tissue or body fluid.

The changes in metabolite concentrations in human blood, urine, or other appropriate biological media over time may be useful in estimating phenol's rate of metabolism in humans. In some instances, the quantification of metabolites may be useful in correlating the exposure doses to the human body burden. 
Studies that correlate phenol exposure with levels of metabolites in human biological matrices are not available for this compound, although analytical methods for the quantification of the metabolites are available.

\section{Methods for Determining Parent Compounds and Degradation Products in Environmental}

Media. The analytical methods available (Eichelberger et al. 1983; EPA 1982, 1986b, 1986c, 1987; Kuwata et al. 1980; Nieminen and Heikkila 1986; NIOSH 1994a, 1994b, 1996; Sithole et al. 1986; Tomkins et al. 1984; Van Rossum and Webb 1978; Yrjanheikki 1978) are adequate for the quantification of phenol in environmental materials. Knowledge of the levels of this compound in environmental media, such as air, water, and food, can be used to indicate exposure of humans to this compound through the inhalation of air and ingestion of drinking water and foods containing phenol.

Although the products of environmental biotic and abiotic degradation of phenol have been identified adequately, no systematic study measuring the concentrations of the degradation products in the environment was found. Analytical methods are available for determining the levels of the degradation products such as hydroxylated phenol. Knowledge of the levels of degradation products would allow the development of a monitoring program designed to assess the ambient concentrations of phenol degradation products in the environment. Such a program could provide information concerning both human and environmental exposure to phenol since it might allow an estimation of the concentration of phenol in the environment prior to degradation.

\subsubsection{Ongoing Studies}

The Federal Research in Progress (FEDRIP 2006) database provides additional information obtainable from a few ongoing studies that may fill in some of the data needs identified in Section 7.3.1. Two studies pertaining to analytical procedures for phenol detection were found in this database. Research being done at Mount Sinai School of Medicine at New York University and supported by National Institute of Environmental Health Sciences is looking into more accurate methods for quantitative detection to toxicants in children and infants. A study from A.D. Grosso sponsored by the Center for Biologics and Evaluation-Quality Control is looking at chromatographic determination of phenol used as an antimicrobial preservative in vaccines and allergenic extracts.

The Environmental Health Laboratory Sciences Division of the National Center for Environmental Health, Centers for Disease Control and Prevention, is developing methods for the analysis of phenol and 
other volatile organic compounds in blood. These methods use purge and trap methodology, highresolution gas chromatography, and magnetic sector mass spectrometry, which give detection limits in the low parts per trillion (ppt) range.

The Environmental Health Laboratory Sciences Division of the National Center for Environmental Health, Centers for Disease Control and Prevention, is developing methods for the analysis of phenol and other phenolic compounds in urine. These methods use high-resolution gas chromatography and magnetic sector mass spectrometry, which give detection limits in the low parts per trillion (ppt) range. 
7. ANALYTICAL METHODS

This page is intentionally blank. 


\section{REGULATIONS AND ADVISORIES}

International and national regulations and guidelines pertinent to human exposure to phenol are summarized in Table 8-1.

ATSDR has derived an acute-duration oral MRL of $1.0 \mathrm{mg} / \mathrm{kg} /$ day based on a BMDL of $125 \mathrm{mg} / \mathrm{kg} / \mathrm{day}$ for changes in body weight gain in pregnant rats exposed to phenol by gavage using divided dosing during GDs 6-15 (York 1997). An uncertainty factor of 100 was used (10 for animal to human extrapolation and 10 for human variability).

EPA (IRIS 2006) derived an oral reference dose (RfD) of $0.3 \mathrm{mg} / \mathrm{kg} /$ day for phenol based on a BMDL of $93 \mathrm{mg} / \mathrm{kg} /$ day for decreased maternal weight gain observed in Sprague-Dawley rats dosed with phenol during gestation (York 1997).

The IARC classification for phenol is Group 3, not classifiable with regard to its carcinogenicity to humans (IARC 2004). The EPA cancer classification for phenol is D, not classifiable as to human carcinogenicity (IRIS 2006). The National Toxicology Program has not classified phenol for human carcinogenicity (NTP 2005). The American Conference of Governmental Industrial Hygienists (ACGIH) has classified phenol as an A4 carcinogen (not classifiable as a human carcinogen) (ACGIH 2005).

OSHA has required employers of workers who are occupationally exposed to phenol to institute engineering controls and work practices to reduce and maintain employee exposure at or below permissible exposure limits (PELs) (OSHA 2005a). The employer must use engineering and work practice controls to reduce exposures to or below an 8-hour time-weighted average (TWA) of 5 ppm for phenol (OSHA 2005a). ACGIH (2005) and NIOSH (2005) also recommend a TWA exposure limit of 5 ppm for occupational exposure.

Phenol is regulated by the Clean Water Effluent Guidelines for the following industrial point sources: electroplating, organic chemicals, steam electric, asbestos, timber products processing, metal finishing, paving and roofing, paint formulating, ink formulating, gum and wood, carbon black, metal molding and casting, aluminum forming, and electrical and electronic components; see the electronic Code of Federal Regulations for a complete listing (NARA 2006). 


\section{Table 8-1. Regulations and Guidelines Applicable to Phenol}

\begin{tabular}{|c|c|c|c|}
\hline Agency & Description & Information & Reference \\
\hline \multicolumn{4}{|c|}{ INTERNATIONAL } \\
\hline \multicolumn{4}{|l|}{ Guidelines: } \\
\hline IARC & Carcinogenicity classification & Group $3^{a}$ & IARC 2004 \\
\hline \multirow[t]{2}{*}{ WHO } & Air quality guidelines & No data & WHO 2000 \\
\hline & Drinking water quality guidelines & No data & WHO 2004 \\
\hline \multicolumn{4}{|l|}{ NATIONAL } \\
\hline \multicolumn{4}{|c|}{$\begin{array}{l}\text { Regulations and } \\
\text { Guidelines: }\end{array}$} \\
\hline \multicolumn{4}{|l|}{ a. Air } \\
\hline ACGIH & TLV (8-hour TWA $)^{\mathrm{b}}$ & 5 ppm & ACGIH 2005 \\
\hline \multirow[t]{14}{*}{ EPA } & AEGL-1 $1^{\mathrm{c}, \mathrm{d}}$ & & EPA $2006 a$ \\
\hline & 10 minutes & 19 ppm & \\
\hline & 30 minutes & 19 ppm & \\
\hline & 60 minutes & 15 ppm & \\
\hline & 4 hours & $9.5 \mathrm{ppm}$ & \\
\hline & 8 hours & $6.3 \mathrm{ppm}$ & \\
\hline & $A E G L-2^{c, d}$ & & \\
\hline & 10 minutes & 29 ppm & \\
\hline & 30 minutes & 29 ppm & \\
\hline & 60 minutes & 23 ppm & \\
\hline & 4 hours & 15 ppm & \\
\hline & 8 hours & 12 ppm & \\
\hline & $A E G L-3^{c, d}$ & $\begin{array}{l}\text { Not recommended due } \\
\text { to insufficient data }\end{array}$ & \\
\hline & Hazardous air pollutant & Yes & $\begin{array}{l}\text { EPA } 2006 d \\
42 \text { USC } 7412\end{array}$ \\
\hline \multirow[t]{3}{*}{$\mathrm{NIOSH}$} & REL (10-hour TWA) ${ }^{\mathrm{e}}$ & $5 \mathrm{ppm}$ & NIOSH 2005 \\
\hline & Ceiling limit (15-minute TWA) & $15.6 \mathrm{ppm}$ & \\
\hline & IDLH & 250 ppm & \\
\hline \multirow[t]{3}{*}{ OSHA } & PEL (8-hour TWA) for general industry ${ }^{\dagger}$ & $5 \mathrm{ppm}$ & $\begin{array}{l}\text { OSHA 2005c } \\
29 \text { CFR } 1910.1000\end{array}$ \\
\hline & $\begin{array}{l}\text { PEL (8-hour TWA) for construction } \\
\text { industry }\end{array}$ & 5 ppm & $\begin{array}{l}\text { OSHA 2005b } \\
29 \text { CFR 1926.55, } \\
\text { Appendix A }\end{array}$ \\
\hline & $\begin{array}{l}\text { PEL (8-hour TWA) for shipyard } \\
\text { industry }^{f}\end{array}$ & $5 \mathrm{ppm}$ & $\begin{array}{l}\text { OSHA 2005a } \\
29 \text { CFR 1915.1000 }\end{array}$ \\
\hline \multicolumn{4}{|l|}{ b. Water } \\
\hline \multirow[t]{2}{*}{ EPA } & $\begin{array}{l}\text { Designated as a hazardous substances } \\
\text { in accordance with Section } 311(b)(2)(A) \\
\text { of the Clean Water Act }\end{array}$ & Yes & $\begin{array}{l}\text { EPA 2006b } \\
40 \text { CFR } 116.4\end{array}$ \\
\hline & $\begin{array}{l}\text { Designated as a toxic pollutant in } \\
\text { accordance with Section } 307(\mathrm{a})(1) \text { of } \\
\text { the Federal Water Pollution Control Act }\end{array}$ & Yes & $\begin{array}{l}\text { EPA 2006c } \\
40 \text { CFR } 401.15\end{array}$ \\
\hline
\end{tabular}




\section{Table 8-1. Regulations and Guidelines Applicable to Phenol}

\begin{tabular}{|c|c|c|c|}
\hline Agency & Description & Information & Reference \\
\hline \multicolumn{4}{|c|}{ NATIONAL (cont.) } \\
\hline \multirow[t]{14}{*}{ EPA } & $\begin{array}{l}\text { Drinking water standards and health } \\
\text { advisories }\end{array}$ & & EPA 2004 \\
\hline & $\begin{array}{l}\text { 1-day health advisory for a } 10-\mathrm{kg} \\
\text { child }\end{array}$ & $6 \mathrm{mg} / \mathrm{L}$ & \\
\hline & $\begin{array}{l}\text { 10-day health advisory for a } 10-\mathrm{kg} \\
\text { child }\end{array}$ & $6 \mathrm{mg} / \mathrm{L}$ & \\
\hline & DWEL & $11 \mathrm{mg} / \mathrm{L}$ & \\
\hline & Lifetime & $2 \mathrm{mg} / \mathrm{L}$ & \\
\hline & $\begin{array}{l}\text { National primary drinking water } \\
\text { standards }\end{array}$ & No data & EPA 2003 \\
\hline & $\begin{array}{l}\text { Reportable quantities of hazardous } \\
\text { substances designated pursuant to } \\
\text { Section } 311 \text { of the Clean Water Act }\end{array}$ & 1,000 pounds & $\begin{array}{l}\text { EPA } 2006 \mathrm{~g} \\
40 \text { CFR } 117.3\end{array}$ \\
\hline & $\begin{array}{l}\text { Toxics criteria for those states not } \\
\text { complying with Clean Water Act }\end{array}$ & & $\begin{array}{l}\text { EPA } 2006 \mathrm{~m} \\
40 \text { CFR } 131.36\end{array}$ \\
\hline & $\begin{array}{l}\text { Section } 303(c)(2)(B) \text { for human health } \\
\left(10^{-6} \text { risk for carcinogens) for }\right. \\
\text { consumption of: }\end{array}$ & & \\
\hline & Water + organism & $21 \mathrm{mg} / \mathrm{L}$ & \\
\hline & Organism only & $4,600 \mathrm{mg} / \mathrm{L}$ & \\
\hline & $\begin{array}{l}\text { Water quality criteria for human health } \\
\text { consumption of: }\end{array}$ & & EPA $2006 f$ \\
\hline & Water + organism & $21 \mathrm{mg} / \mathrm{L}$ & \\
\hline & Organism only & $1,700 \mathrm{mg} / \mathrm{L}$ & \\
\hline \multicolumn{4}{|l|}{ c. Food } \\
\hline \multirow[t]{2}{*}{ EPA } & $\begin{array}{l}\text { Exemptions from the requirement of a } \\
\text { tolerance as an inert ingredient (as a } \\
\text { solvent) when used pre-harvest }\end{array}$ & Yes & $\begin{array}{l}\text { EPA } 2006 \mathrm{k} \\
40 \text { CFR } 180.920\end{array}$ \\
\hline & $\begin{array}{l}\text { Exemptions from the requirement of a } \\
\text { tolerance as an inert ingredients (as a } \\
\text { solvent) when applied to animals }\end{array}$ & Yes & $\begin{array}{l}\text { EPA 2006I } \\
40 \text { CFR } 180.930\end{array}$ \\
\hline \multirow[t]{2}{*}{ FDA } & Bottled drinking water & $0.001 \mathrm{mg} / \mathrm{L}$ & FDA 2005 \\
\hline & $\begin{array}{l}\text { Included on the "Everything Added to } \\
\text { Foods in the United States" List }\end{array}$ & Yes & FDA 2006 \\
\hline \multicolumn{4}{|l|}{ d. Other } \\
\hline \multirow[t]{2}{*}{ ACGIH } & Carcinogenicity classification & $\mathrm{A} 4^{\mathrm{g}}$ & ACGIH 2005 \\
\hline & $\begin{array}{l}\text { Biological exposure indices (end of } \\
\text { shift) for total phenol in urine }\end{array}$ & 250 mg/g creatinine & \\
\hline CPSC & $\begin{array}{l}\text { Substance named in the Federal } \\
\text { Caustic Poison Act; phenol and any } \\
\text { preparation containing phenol in a } \\
\text { concentration }\end{array}$ & $\leq 5 \%$ & CPSC 2005 \\
\hline EPA & $\begin{array}{l}\text { Carcinogenicity classification } \\
\text { Oral slope factor } \\
\text { Inhalation unit risk }\end{array}$ & $\begin{array}{l}\text { Group } D^{\mathrm{n}} \\
\text { Not applicable } \\
\text { Not applicable }\end{array}$ & IRIS 2006 \\
\hline
\end{tabular}




\section{Table 8-1. Regulations and Guidelines Applicable to Phenol}

\begin{tabular}{|c|c|c|c|}
\hline Agency & Description & Information & Reference \\
\hline \multicolumn{4}{|c|}{ NATIONAL (cont.) } \\
\hline & $\mathrm{RfC}$ & Not applicable & IRIS 2006 \\
\hline & RfD & $0.3 \mathrm{mg} / \mathrm{kg} / \mathrm{day}$ & \\
\hline & $\begin{array}{l}\text { Identification and listing of hazardous } \\
\text { waste; hazardous waste number }\end{array}$ & U188 & $\begin{array}{l}\text { EPA 2006e } \\
40 \text { CFR 261, } \\
\text { Appendix VIII }\end{array}$ \\
\hline & $\begin{array}{l}\text { Superfund, emergency planning, and } \\
\text { community right-to-know }\end{array}$ & & \\
\hline & $\begin{array}{l}\text { Designated CERCLA hazardous } \\
\text { substance }\end{array}$ & Yes & $\begin{array}{l}\text { EPA } 2006 \mathrm{~h} \\
40 \text { CFR } 302.4\end{array}$ \\
\hline & Reportable quantity & 1,000 pounds & \\
\hline & $\begin{array}{l}\text { Effective date of toxic chemical } \\
\text { release reporting }\end{array}$ & $01 / 01 / 87$ & $\begin{array}{l}\text { EPA 2006j } \\
40 \text { CFR } 372.65\end{array}$ \\
\hline & $\begin{array}{l}\text { Extremely hazardous substances } \\
\text { and their threshold planning } \\
\text { quantities }\end{array}$ & $500 / 10,000$ pounds & $\begin{array}{l}\text { EPA } 2006 \mathrm{i} \\
40 \text { CFR 355, } \\
\text { Appendix A }\end{array}$ \\
\hline NTP & Carcinogenicity classification & No data & NTP 2005 \\
\hline
\end{tabular}

${ }^{a}$ Group 3: not classifiable as to carcinogenicity to humans

bSkin notation: refers to the potential significant contribution to the overall exposure by the cutaneous route, including mucous membranes and the eyes, either by contact with vapors, liquids, or solids.

${ }^{\mathrm{c}} \mathrm{AEGL}-1$ is the airborne concentration of a substance above which it is predicted that the general population, including susceptible individuals, could experience notable discomfort, irritation, or certain asymptomatic nonsensory effects. AEGL-2 is the airborne concentration of a substance above which it is predicted that the general population, including susceptible individuals, could experience irreversible or other serious, long-lasting adverse health effects or an impaired ability to escape. AEGL-3 is the airborne concentration of a substance above which it is predicted that the general population, including susceptible individuals, could experience life-threatening health effects or death. ${ }^{\mathrm{d}}$ Level of distinct odor awareness $=0.25 \mathrm{ppm}$

e Skin designation: indicates the potential for dermal absorption; skin exposure should be prevented as necessary through the use of good work practices, gloves, coveralls, goggles, and other appropriate equipment.

fSkin designation

${ }^{g}$ A4: not classifiable as a human carcinogen

${ }^{\mathrm{h}}$ Group D: not classifiable as to human carcinogenicity

'Not applicable: no adequate inhalation exposure studies exist from which an inhalation RfC may be derived. A route-to-route extrapolation is not appropriate, because phenol can be a direct contact irritant, and so portal-of-entry effects are a potential concern.

ACGIH = American Conference of Governmental Industrial Hygienists; AEGL = Acute Exposure Guideline Level; CERCLA = Comprehensive Environmetnal Response, Compensation, and Liability Act; CFR = Code of Federal Regulations; CPSC = Consumer Product Safety Commission; DWEL = drinking water equivalent level;

$\mathrm{EPA}=$ Environmental Protection Agency; FDA = Food and Drug Administration; IARC = International Agency for Research on Cancer; IDLH = immediately dangerous to life or health; IRIS = Integrated Risk Information System; $\mathrm{NIOSH}=$ National Institute for Occupational Safety and Health; NTP = National Toxicology Program;

OSHA = Occupational Safety and Health Administration; PEL = permissible exposure limit; REL = recommended exposure limit; $\mathrm{RfC}=$ inhalation reference concentration; $\mathrm{RfD}=$ oral reference dose; $T L V=$ threshold limit values; TWA = time-weighted average; USC = United States Code; WHO = World Health Organization 
EPA regulates phenol under the Clean Water Act (CWA) and the Clean Air Act (CAA) and has designated it as a hazardous substance and a hazardous air pollutant (HAP) (EPA 2006b, 2006c). Phenol is on the list of chemicals appearing in "Toxic Chemicals Subject to Section 313 of the Emergency Planning and Community Right-to-Know Act of 1986" (EPA 2006j) and has been assigned a reportable quantity (RQ) limit of 1,000 pounds (EPA 2006h). The RQ represents the amount of a designated hazardous substance which, when released to the environment, must be reported to the appropriate authority. Phenol is also considered to be an extremely hazardous substance (EPA 2006i). 
8. REGULATIONS AND ADVISORIES

This page is intentionally blank. 


\section{REFERENCES}

ACGIH. 2001. Documentation of the threshold limit values and biological exposure indices. Phenol. 7th ed. Cincinnati, OH: American Conference of Governmental Industrial Hygienists.

ACGIH. 2005. Threshold limit values for chemical substances and physical agents and biological indices. Cincinnati, OH: American Conference of Government Industrial Hygienists.

Achilli G, Cellerino GP, d'Eril GM, et al. 1995. Simultaneous determination of 27 phenols and herbicides in water by high-performance liquid chromatography with multi-electrode electrochemical detection. J Chromatogr 697:357-362.

Adlercreutz H. 1995. Phytoestrogens: Epidemiology and a possible role in cancer protection. Environ Health Perspect Suppl 103(7):103-112.

Adinolfi M. 1985. The development of the human blood-csf-brain barrier. Dev Med Child Neurol 27:532-537.

Agency for Toxic Substances and Disease Registry. 1989. Decision guide for identifying substancespecific data needs related to toxicological profiles. Atlanta, GA: Agency for Toxic Substances and Disease Registry, Division of Toxicology.

Agency for Toxic Substances and Disease Registry. 1990. Biomarkers of organ damage or dysfunction for the renal, hepatobiliary, and immune systems. Subcommittee on Biomarkers of Organ Damage and Dysfunction. Atlanta, GA: Agency for Toxic Substances and Disease Registry.

Agency for Toxic Substances and Disease Registry. 2006. Case studies in environmental medicine. Benzene toxicity. Atlanta, GA: Agency for Toxic Substances and Disease Registry. www.atsdr.cdc.gov. March 24, 2006.

Ahmed N, Hale K. 1994. A microassay for urinary phenol using capillary gas chromatography and optimised enzymic hydrolysis. Clin Chim Acta 230:201-208.

Al-Harazin IM, Nakhla GF, Farooq S. 1991. Start-up of sequencing batch reactors for toxic wastewater treatment. J Environ Sci Health A26:673-687.

Altman PK, Dittmer DS. 1974. Biological handbooks: Biology data book. Volume III, 2nd ed. Bethesda, MD: Federation of American Societies for Experimental Biology, 1987-2008, 2041.

Amlacher E, Rudolph C. 1981. The thymidine incorporation inhibiting screening system (TSS) to test carcinogenic substances (a nuclear DNA synthesis suppressive short term test). Arch Geschwulstforsch 51:605-610.

Amlathe S, Upadhyay S, Gupta VK. 1987. Spectrophotometric determination of trace amounts of phenol in wastewater and biological fluids. Analyst 112:1463-1465.

\footnotetext{
* Not cited in text
} 
Amoore JE, Hautala E. 1983. Odor as an aid to chemical safety: Odor thresholds compared with threshold limit values and volatilities for 214 industrial chemicals in air and water dilution. J Appl Toxicol 3:272-290.

Andersen ME, Clewell HJ,III, Gargas ML, et al. 1987. Physiologically-based pharmacokinetics and the risk assessment process for methylene chloride. Toxicol Appl Pharmacol 87:185-205.

Andersen ME, Krishnan K. 1994. Relating in vitro to in vivo exposures with physiologically-based tissue dosimetry and tissue response models. In: Salem H, ed. Animal test alternatives. Aberdeen Proving Ground, MD: U.S. Army Chemical Research Development and Engineering Center.

APHA. 1998a. Method 5530c. Chloroform extraction method. In: Clesceri LS, Greenberg AE, Eaton AD, eds. Standard methods for the examination of water and wastewater. 20th. Washington, DC: American Public Health Association. American Water Works Association. Water Environmental Federation, 5-45 to 5-43.

APHA. 1998b. Method 6420B. Liquid-liquid extraction gas chromatographic method. In: Clesceri LS, Greenberg AE, Eaton AD, eds. Standard methods for the examination of water and wastewater. 20th. Washington, DC: American Public Health Association. American Water Works Association. Water Environmental Federation, 6-73 to 6-78.

Aranyi C, O'Shea WJ, Graham JA, et al. 1986. The effects of inhalation of organic chemical air contaminants on murine lung host defenses. Fundam Appl Toxicol 6:713-720.

Artiola-Fortuny J, Fuller WH. 1982. Adsorption of some monohydroxybenzene derivatives by soils. Soil Sci 133(1):18-26.

Atkinson R, Aschmann SM, Winer AM. 1987. Kinetics of the reactions of $\mathrm{NO}_{3}$ radicals with a series of aromatic compounds. Environ Sci Technol 21:1123-1126.

Axelsson G, Lutz C, Rylander R. 1984. Exposure to solvents and outcome of pregnancy in university laboratory employees. Br J Ind Med 41:303-312.

Baj Z, Majewska E, Zeman K, et al. 1994. The effect of chronic exposure to formaldehyde, phenol and organic chlorohydrocarbons on peripheral blood cells and the immune system in humans. J Invest Allergol Clin Immunol 4:186-191.

Baker MD, Mayfield CI. 1980. Microbial and nonbiological decomposition of chlorophenols and phenol in soil. Water Air Soil Pollut 13:411-424.

Baker EL, Landrigan PJ, Bertozzi PE, et al. 1978. Phenol poisoning due to contaminated drinking water. Arch Environ Health 33:89-94.

Baldwin MK, Selby MA, Bloomberg H. 1981. Measurement of phenol in urine by the method of Van Haaften and Sie: A critical appraisal. Analyst 106:763-767.

Ballinger LN, Cross SE, Roberts MS. 1995. Availability and mean transit times of phenol and its metabolites in the isolated perfused rat liver: Normal and retrograde studies using tracer concentrations of phenol. J Pharm Pharmacol 47:949-956. 
Barale R, Marrazzini A, Betti C, et al. 1990. Genotoxicity of two metabolites of benzene: Phenol and hydroquinone show strong synergistic effects in vivo. Mutat Res 244:15-20.

Baranowska-Dutkiewicz B. 1981. Skin absorption of phenol from aqueous solutions in men. Int Arch Occup Environ Health 49:99-104.

Barnes DG, Dourson M. 1988. Reference dose (RfD): Description and use in health risk assessments. U.S. Environmental Protection Agency. Regul Toxicol Pharmacol 8:471-486.

Behl CR, Linn EE, Flynn GL, et al. 1983. Permeation of skin and eschar by antiseptics. I: Baseline studies with phenol. J Pharm Sci 72:391-397.

Bentur Y, Shoshani O, Tabak A, et al. 1998. Prolonged elimination of half-life of phenol after dermal exposure. Clin Toxicol 36(7):707-711.

Berger GS. 1994. Epidemiology of endometriosis. In: Berger GS, ed. Endometriosis: Advanced management and surgical techniques. New York, NY: Springer-Verlag, 3-7.

Berman E, Schlicht M, Moser VC, et al. 1995. A multidisciplinary approach to toxicological screening: I. Systemic toxicity. J Toxicol Environ Health 45:127-143.

Beveridge T. 1997. Haze and cloud in apple juices. Crit Rev Food Sci Nutr 37:75-91.

Beyrouty P. 1998. Final report: A 13-week neurotoxicity study of phenol administered in the drinking water to the rat, with TSCA notice of receipt of test data. Chemical Manufacturers Association. Submitted to U.S. Environmental Protection Agency under TSCA Section 4. OTS0559602.

Bieniek G. 1994. Concentrations of phenol, o-cresol, and 2,5-xylenol in the urine of workers employed in the distillation of the phenolic fraction of tar. Occup Environ Med 51:354-356.

Bieniek G, Wilczok T. 1986. Separation and determination of phenol, $\alpha$-naphthol, $m$ - and $p$-, $o$-cresols and 2,5-xylenol and catechol in the urine after mixed exposure to phenol, naphthalene, cresols and xylenols. Br J Ind Med 43:570-571.

Boatto G, Nieddu M, Carta A, et al. 2004. Determination of phenol and $o$-cresol by GC/MS in a fatal poisoning case. Forensic Sci Int 139(2-3):191-194.

Bois FY, Smith MT, Spear RC. 1991. Mechanisms of benzene carcinogenesis: Application of a physiological model of benzene pharmacokinetics and metabolism. Toxicol Lett 56:283-298.

Boutwell RK, Bosch DK. 1959. The tumor-promoting action of phenol and related compounds for mouse skin. Cancer Res 19:413-424.

Boyd SA. 1982. Adsorption of substituted phenols by soil. Soil Sci 134:337-343.

Boyd SA, Shelton DR, Berry D, et al. 1983. Anaerobic biodegradation of phenolic compounds in digested sludge. Appl Environ Microbiol 46:50-54.

Brandorff NP, Flyvhokm M-A, Beck ID, et al. 1995. National survey on the use of chemicals in the working environment. Estimated exposure events. Occup Environ Med 52:454-463. 
Briggs GG. 1981. Theoretical and experimental relationships between soil adsorption, octanol-water partition coefficients, water solubilities, bioconcentration factors and the parachor. J Agric Food Chem 29:1050-1059.

Brown VKH, Box VL, Simpson BJ. 1975. Decontamination procedures for skin exposed to phenolic substances. Arch Environ Health 30:1-6.

Bruce RM, Santodonato J, Neal MW. 1987. Summary review of the health effects associated with phenol. Toxicol Ind Health 3:535-568.

Budavari S, O'Neil MJ, Smith A, et al., eds. 1989. Phenol. The Merck index, 11th ed. Rahway, NJ: Merck \& Co., Inc., 1150.

Bulsiewicz H. 1977. The influence of phenol on chromosomes of mice (Mus musculus) in the process of spermatogenesis. Folia Morphol (Warsaw) 36:13-22.

Burchell B, Coughtrie MWH. 1997. Genetic and environmental factors associated with variation of human xenobiotic glucuronidation and sulfation. Environ Health Perspect 105:739-747.

Buxton ILO. 2006. Pharmacokinetics and pharmacodynamics. The dynamics of drug absorption, distribution, action, and elimination. In: Brunton LL, Lazo JS, Parker KL, eds., Goldman \& Gilman's the pharmacological basis of therapeutics. $11^{\text {th }}$ ed. New York, NY: McGraw-Hill, 1-39.

Cal EPA. 2003. Consideration of chemicals known to the state to cause reproductive toxicity. Phenol. In: Staff presentations for developmental and reproductive toxicant identification committee meeting held on October 19, 2003. California Environmental Protection Agency, Office of Environmental Health Hazard Assessment. http://www.oehha.ca.gov/prop65/public_meetings/101603dartmeetmat.html. January 18, 2008.

Campbell N, Van Loon JA, Weinshilboum RM. 1987. Human liver phenol sulfotransferase: Assay conditions, biochemical properties and partial purification of isozymes of the thermostable form. Biochem Pharmacol 36:1435-1446.

Canonica S, Jans U, Stemmler K, et al. 1995. Transformation kinetics of phenols in water: Photosensitization by dissolved natural organic material and aromatic ketones. Environ Sci Technol 29:1822-1831.

Capel ID, French MR, Millburn P, et al. 1972. Fate of $\left[{ }^{14} \mathrm{C}\right]-$ phenol in various species. Xenobiotica 2:25-34.

Cassidy MK, Houston JB. 1984. In vivo capacity of hepatic and extra hepatic enzymes to conjugate phenol. Drug Metab Dispos 12:619-624.

Chapman DE, Namkung MJ, Juchau MR. 1994. Benzene and benzene metabolites as embryotoxic agents: Effects on cultured rat embryos. Toxicol Appl Pharmacol 128(1):129-137.

Chen CS, Zoltek J Jr. 1995. Organic priority pollutants in wetland-treated leachates at a landfill in central Florida. Chemosphere 31:3455-3464. 
Chen H, Eastmond DA. 1995a. Synergistic increase in chromosomal breakage within the euchromatin induced by an interaction of the benzene metabolites phenol and hydroquinone in mice. Carcinogenesis 16:1963-1969.

Chen H, Eastmond DA. 1995b. Topoisomerase inhibition by phenolic metabolites: A potential mechanism for benzene's clastogenic effects. Carcinogenesis 16:2301-2307.

Chowdhury JR, Novikoff PM, Chowdhury NR, et al. 1985. Distribution of UDPglucuronosyltransferase in rat tissue. Proc Natl Acad Sci USA 82:2990-2994.

Chung HY. 1999. Volatile components in fermented soybean (Glycine max) curds. J Agric Food Chem 47:26902697.

Ciranni R, Barale R, Marrazzini A, et al. 1988. Benzene and the genotoxicity of its metabolites. I. Transplacental activity in mouse fetuses and in their dams. Mutat Res 208:61-67.

Clark TP, Piskin R. 1977. Chemical quality and indicator parameters for monitoring landfill leachate in Illinois. Environ Geol 1:329-340.

Clewell HJ, III, Andersen ME. 1985. Risk assessment extrapolations and physiological modeling. Toxicol Ind Health 1:111-113.

CMA. 1998. Two-week (ten day) inhalation toxicity and two-week recovery study of phenol vapor in the rat. Chemical Manufacturers Association. Submitted to U.S. Environmental Protection Agency under TSCA Section 4. OTS0559328.

CMR. 2005. Phenol: Chemical Profile. Chemical Market Reporter, 34-35. May 23-29, 2005.

Coan ML, Baggs RB, Bosmann HB. 1982. Demonstration of direct toxicity of phenol on kidney. Res Commun Chem Pathol Pharmacol 36:229-239.

Cole RH, Frederick RE, Healy RP, et al. 1984. Preliminary findings of the priority pollutant monitoring project of the nationwide urban runoff program. J Water Pollut Contr Fed 56:898-908.

Conning DM, Hayes MJ. 1970. The dermal toxicity of phenol: An investigation of the most effective first-aid measures. Br J Ind Med 27:155-159.

Constan AA, Yang RSH, Baker DC, et al. 1995. A unique pattern of hepatocyte proliferation in F344 rats following long-term exposures to low levels of a chemical mixture of groundwater contaminants. Carcinogenesis 16:303-310.

CPSC. 2005. Hazardous substances and articles: Administration and enforcement regulations. Substances named in the Federal Caustic Poison Act. Washington, DC: Consumer Product Safety Commission. Code of Federal Regulations. 15 CFR 1500.129. http://www.access.gpo.gov/nara/cfr/cfrtable-search.html\#page1. March 08, 2006.

Crawford DW, Bonnevie NC, Wenning RJ. 1995. Sources of pollution and sediment contamination in Newark Bay, New Jersey. Ecotoxicol Environ Safety, 30:85-100.

Crebelli R, Conti G, Carere A. 1987. On the mechanism of mitotic segregation induction in Aspergillus nidulans by benzene hydroxy metabolites. Mutagenesis 2:235-238. 
Cronin TD, Brauer RO. 1949. Death due to phenol contained in foille. JAMA 139:777-778.

Cronn DR, Charlson RJ, Knights RL, et al. 1977. A survey of the molecular nature of primary and secondary components of particles in urban air by high-resolution mass spectrometry. Atmos Environ 11:929-937.

Dalin NM, Kristoffersson R. 1974. Physiological effects of a sublethal concentration of inhaled phenol on the rat. Ann Zool Fenn 11:193-199.

Darisimall. 2006. Sore throat lozenges and sprays. http://store.darisimall.com/sothlosp.html. July 27, 2006.

Davies MI, Lunte CE. 1996. Simultaneous microdialysis sampling from multiple sites in the liver for the study of phenol metabolism. Life Sci 59:1001-1013.

Davis EM, Murray HE, Liehr JG, et al. 1981. Basic microbial degradation and chemical byproducts of selected organic compounds. Water Res 15:1125-1127.

De Ceaurriz JC, Micillino JC, Bonnet P, et al. 1981. Sensory irritation caused by various industrial airborne chemicals. Toxicol Lett (Amst) 9:137-144.

Deichmann WB. 1944. Phenol studies. V. The distribution, detoxification, and excretion of phenol in the mammalian body. Arch Biochem 3:345-355.

Deichmann WB, Keplinger ML. 1981. Phenols and phenolic compounds. In: Clayton GD, Clayton FE, eds. Patty's industrial hygiene and toxicology, 3rd ed. New York, NY: John Wiley and Sons, Inc., 25672627.

Deichmann WB, Witherup S. 1944. Phenol studies. VI: The acute and comparative toxicity of phenol and $o-, m$-, and $p$-cresols for experimental animals. J Pharmacol Exp Ther 80:233-240.

Deichmann WB, Kitzmiller KV, Witherup BS. 1944. Phenol studies. VII. Chronic phenol poisoning, with special reference to the effects upon experimental animals of the inhalation of phenol vapor. Am J Clin Pathol 14:273-277.

Delfino JJ, Dube DJ. 1976. Persistent contamination of ground water by phenol. J Environ Sci Health A11:345-355.

*Della Pietra G, Illiano G, Soscia M. 1967. Glycuronic acid and glycuronamide detoxicating activity. Biochem Pharmacol 16:1571-1573.

Demerec M, Bertran G, Flint J. 1951. A survey of chemicals for mutagenic action on E. coli. American Naturalist 85:119-136.

Di Corcia A, Bellioni A, Madbouly MD, et al. 1996. Trace determination of phenols in natural waters. Extraction by a new graphitized carbon black cartridge followed by liquid chromatography and reanalysis after phenol derivatization. J Chromatogr 733:383-393.

Doan HM, Keith L, Shennan AT. 1979. Phenol and neonatal jaundice. Pediatrics 64:324-325. 
DOE. 1997. Method OM100R: Semivolatile organic compounds in multimedia samples by capillary column ion trap MS. Idaho Falls, ID: U.S. Department of Energy, National Analytical Management Program. http://web1.er.usgs.gov/nemi/method_pdf/4795.pdf. April 18, 2006.

DOE. 1987. Uptake and fate of phenol aniline and quinoline in terrestrial plants. In: Gray RH, ed. Health and environmental research on complex organic mixtures. U.S. Department of Energy, 631-641.

Dosemeci M, Blair A, Stewart PA, et al. 1991. Mortality among industrial workers exposed to phenol. Epidemiology 2:188-193.

Douglas CC. 1972. Gas chromatographic determination of phenolic compounds in drug preparations: Collaborative study. J Assoc Off Anal Chem 55:610-612.

*Ducis I, Norenberg L-OB, Norenberg MD. 1990. Effect of phenol and sodium octanoate on the astrocyte benzodiazepine receptor. Brain Res 514:349-351.

Dumont JP, Adda J. 1978. Occurrence of sesquiterpenes in mountain cheese volatiles. J Agric Food Chem 26:364-367.

Durback-Morris LF, Scharman EJ. 1999. Accidental intranasal administration of phenol. Vet Hum Toxicol 41(3):157.

Eastmond DA, Smith MT, Ruzo LO, et al. 1986. Metabolic activation of phenol by human myeloperoxidase and horseradish peroxidase. Mol Pharmacol 30:674-679.

Edwards RD, Jurvelin J, Saarela K, et al. 2001. VOC concentrations measured in personal samples and residential indoor, outdoor and workplace microenvironments in EXPOLIS- Helsinki, Finland. Atmos Environ 35(27):4829-4841.

Edwards VT, Jones BC, Hutson DH. 1986. A comparison of the metabolic fate of phenol, phenyl glucoside and phenyl 6-O-malonyl-glucoside in the rat. Xenobiotica 16:801-807.

Ehrlich GG, Goelitz DF, Godsy EM, et al. 1982. Degradation of phenolic contaminants in ground water by anaerobic bacteria: St. Louis Park, MN. Ground Water 20:703-710.

Eichelberger JW, Kerns EH, Olynyk P, et al. 1983. Precision and accuracy in the determination of organics in water by fused silica capillary column gas chromatography/mass spectrometry and packed column gas chromatography/mass spectrometry. Anal Chem 55:1471-1479.

Elder VA, Proctor BL, Hites RA. 1981. Organic compounds found near dump sites in Niagara Falls, NY. Environ Sci Technol 15:1237-1243.

EPA. 1979a. Atmospheric reaction products of organic compounds. Washington, DC: U.S. Environmental Protection Agency, Office of Toxic Substances. EPA5601279001. PB301384.

EPA. 1979b. Microbial degradation of organochlorine compounds in estuarine waters and sediments. In: Proceedings Workshop: Microbial Degradation Pollution Marine Environment, 1978. Washington, DC: U.S. Environmental Protection Agency, Office of Research and Development. EPA600979012.

EPA. 1979c. Source assessment: Manufacture of acetone and phenol from cumene. Research Triangle Park, NC: U.S. Environmental Protection Agency. EPA600279019d. PB80150592. 
EPA. 1980. Ambient water quality criteria document for phenol. Washington, DC: U.S. Environmental Protection Agency. EPA440580001A. PB81117772.

EPA. 1981a. Exposure and risk assessment for phenol (revised). Washington, DC: U.S. Environmental Protection Agency. PB85221695.

EPA. 1981b. Treatability manual. Washington, DC: U.S. Environmental Protection Agency, Office of Research and Development. EPA600282001A, I.8. 1-1-1-5.

EPA. 1982. Test methods. Methods for organic chemical analysis of municipal and industrial wastewater. Method No. 604 and 625. Cincinnati, OH: U.S. Environmental Protection Agency.

EPA. 1986a. Reference values for risk assessment. Cincinnati, OH: U.S. Environmental Protection Agency. Environmental Criteria and Assessment Office, Office of Health and Environmental Assessment.

EPA. 1986b. Method 8250: Gas chromatography/mass spectrometry for semivolatile organics: Packed column technique. Test methods for evaluating solid waste. SW-846. 3rd ed. Washington, DC: U.S. Environmental Protection Agency, Office of Solid Waste and Emergency Response.

EPA. 1986c. Method 8270: Gas chromatography/mass spectrometry for semivolatile organics: Capillary column technique. Test methods for evaluating solid waste. SW-846. 3rd ed. Washington, DC: U.S. Environmental Protection Agency, Office of Solid Waste and Emergency Response.

EPA. 1987. Health and environmental effects profile for phenol. Cincinnati, OH: U.S. Environmental Protection Agency, Office of Solid Waste and Emergency Response.

EPA. 1994a. Methods for derivation of inhalation reference concentrations and application of inhalation dosimetry. Washington, DC: U.S. Environmental Protection Agency. Office of Research and Development. EPA6008900066F.

EPA. 1994b. Semivolatile organic compounds by gas chromatography/mass spectrometry. Method 8250A. In: Test methods for evaluating solid waste. SW-846. Washington, DC: U.S. Environmental Protection Agency, Office of Solid Waste and Emergency Response.

EPA. 1997. Special report on environmental endocrine disruption: An effects assessment and analysis. Washington, DC: U.S. Environmental Protection Agency, Risk Assessment Forum. EPA630R96012.

EPA. 1998. Method 8070D: Semivolatile organic compounds by GC/MS. Draft update IVA of SW-846 on-line. U.S. Environmental Protection Agency, Office of Solid Waste.

http://www.epa.gov/epaoswer/hazwaste/test/pdfs/8270d.pdf. April 18, 2006

EPA. 1999. Evaluation and application of methods for estimating children's exposure to persistent organic pollutants in multiple media. Research Triangle Park, NC: U.S. Environmental Protection Agency, Office of Research and Development. PB99134942.

EPA. 2000a. Method 528: Determination of phenols in drinking water by solid phase extraction and capillary column gas chromatography/mass spectrometry (GC/MS) Methods for the determination of organic and inorganic compounds in drinking water, Volume 1. Washington, DC: U.S. Environmental Protection Agency. EPA815R00014. http://nepis.epa.gov/pubtitleOW.htm. April 18, 2006. 
EPA. 2000b. Method 8041A: Phenols in aqueous and non-aqueous samples by capillary GC/FID or GC/ECD and single or dual columns. Draft update IVB of SW-846 on-line. U.S. Environmental Protection Agency, Office of Solid Waste. http://www.epa.gov/epaoswer/hazwaste/test/pdfs/8041a.pdf. April 18, 2006.

EPA. 2000c. Benchmark dose technical guidance document. Washington, DC: U.S. Environmental Protection Agency. EPA630R00001.

EPA. 2001a. Method 604: Phenols. U.S. Environmental Protection Agency. Code of Federal Regulations. 40 CFR Part 136, Appendix A. http://web1.er.usgs.gov/nemi/method_pdf/4789.pdf. April $18,2006$.

EPA. 2001b. Method 1625: Semivolatile organic compounds by isotope dilution GCMS. U.S. Environmental Protection Agency. Code of Federal Regulations. 40 CFR Part 136, Appendix A. http://web1.er.usgs.gov/nemi/method_pdf/4686.pdf. April 18, 2006.

EPA. 2001c. Method 635: Base/neutrals and acids. U.S. Environmental Protection Agency. Code of Federal Regulations. 40 CFR 136, Appendix A. http://web1.er.usgs.gov/nemi/method_pdf/4714.pdf. April 20, 2006.

EPA. 2002. Toxicological review of phenol. In support of summary information on the Integrated Risk Information System (IRIS). Washington, DC: U.S. Environmental Protection Agency. EPA635R02006.

EPA. 2003. National primary drinking water regulations. Washington, DC: U.S. Environmental Protection Agency, Office of Ground Water and Drinking Water. EPA816F03016. http://epa.gov/safewater/mcl.html. March 07, 2006.

EPA. 2004. Drinking water standards and health advisories. Washington, DC: U.S. Environmental Protection Agency, Office of Water. EPA822R04005. http://epa.gov/waterscience/criteria/drinking/. March 07, 2006.

EPA. 2005. Toxic chemical release inventory reporting forms and instructions: Revised 2004 version. Section 313 of the Emergency Planning and Community Right-to-Know Act (Title III of the Superfund Amendments and Reauthorization Act of 1986). U.S. Environmental Protection Agency. Office of Environmental Information. EPA260B05001.

EPA. 2006a. Acute exposure guideline levels (AEGLs). Washington, DC: U.S. Environmental Protection Agency, Office of Pollution Prevention and Toxics. http://epa.gov/oppt/aegl/chemlist.htm. March 22, 2006.

EPA. 2006b. Designated as hazardous substances in accordance with Section 311(b)(2)(A) of the Clean Water Act. Washington, DC: U.S. Environmental Protection Agency. Code of Federal Regulations. 40 CFR 116.4.

http://a257.g.akamaitech.net/7/257/2422/22jul20061500/edocket.access.gpo.gov/cfr_2006/julqtr/pdf/40cf r116.4.pdf. January 8, 2008.

EPA. 2006c. Effluent guidelines and standards. Toxic pollutants. Washington, DC: U.S.

Environmental Protection Agency. Code of Federal Regulations. 40 CFR 401.15.

http://www.epa.gov/epacfr40/chapt-1.info/chi-toc.htm. March 08, 2006. 
EPA. 2006d. Hazardous air pollutants. Clean Air Act. Washington, DC: U.S. Environmental Protection Agency. United States Code 42 USC 7412. http://www.epa.gov/ttn/atw/orig189.html. March 07, 2006.

EPA. 2006e. Identification and listing of hazardous waste. Washington, DC: U.S. Environmental Protection Agency. Code of Federal Regulations. 40 CFR 261, Appendix VIII. http://www.epa.gov/epacfr40/chapt-1.info/chi-toc.htm. March 07, 2006.

EPA. 2006f. National recommended water quality criteria. Washington, DC: U.S. Environmental Protection Agency, Office of Water, Office of Science and Technology. http://www.epa.gov/waterscience/criteria/nrwqc-2006.pdf. January 8, 2008.

EPA. 2006g. Table 117.3. Reportable quantities of hazardous substances designated pursuant to Section 311 of the Clean Water Act. Washington, DC: U.S. Environmental Protection Agency. Code of Federal Regulations 40 CFR 117.3.

http://a257.g.akamaitech.net/7/257/2422/22ju120061500/edocket.access.gpo.gov/cfr_2006/julqtr/pdf/40cf r117.3.pdf. January 08, 2008.

EPA. 2006h. Designation of hazardous substances. Washington, DC: U.S. Environmental Protection Agency. Code of Federal Regulations. 40 CFR 302.4.

http://a257.g.akamaitech.net/7/257/2422/22jul20061500/edocket.access.gpo.gov/cfr_2006/julqtr/pdf/40cf r302.4.pdf. January 08, 2008.

EPA. 2006i. Superfund, emergency planning, and community right-to-know programs. Extremely hazardous substances and their threshold planning quantities. Washington, DC: U.S. Environmental Protection Agency. Code of Federal Regulations. 40 CFR 355, Appendix A. http://www.epa.gov/epacfr40/chapt-1.info/chi-toc.htm. March 08, 2006.

EPA. 2006j. Toxic chemical release reporting: Community right-to-know. Chemicals and chemical categories to which this part applies. Washington, DC: U.S. Environmental Protection Agency. Code of Federal Regulations. 40 CFR 372.65.

http://a257.g.akamaitech.net/7/257/2422/22jul20061500/edocket.access.gpo.gov/cfr_2006/julqtr/pdf/40cf r372.65.pdf. January 08, 2008.

EPA. 2006k. Tolerances and exemptions from tolerances for pesticide chemicals in food. Aluminum sulfate. Phenol. Washington, DC: U.S. Environmental Protection Agency. Code of Federal Regulations. 40 CFR 180.920. http://www.epa.gov/epacfr40/chapt-1.info/chi-toc.htm. March 08, 2006.

EPA. 20061. Tolerances and exemptions from tolerances for pesticide chemicals in food. Phenol. Aluminum oxide. Washington, DC: U.S. Environmental Protection Agency. Code of Federal Regulations. 40 CFR 180.930. http//www.epa.gov/epacfr40/chapt-1. March 08, 2006.

EPA. 2006m. Water quality standards. Toxics criteria for those states not complying with Clean Water Act Section 303(c)(2)(B). Washington, DC: U.S. Environmental Protection Agency. Code of Federal Regulations. 40 CFR 131.36. http://www.epa.gov/epacfr40/chapt-1.info/chi-toc.htm. March 08, 2006.

EPA. 2007. Method 8041A. Phenols by gas chromatography. Test methods for evaluation solid waste. U.S. Environmental Protection Agency. http://www.epa.gov/epaoswer/hazwaste/test/pdfs/8041a.pdf. July 31, 2008. 


\section{REFERENCES}

Erexson GL, Wilmer JL, Kligerman AD. 1985. Sister chromatid exchange induction in human lymphocytes exposed to benzene and its metabolites in vitro. Cancer Res 45:2471-2477.

FDA. 2005. Beverages. Bottled water. Washington, DC: U.S. Food and Drug Administration. Code of Federal Regulations. 21 CFR 165.110.

http://a257.g.akamaitech.net/7/257/2422/01apr20051500/edocket.access.gpo.gov/cfr_2005/aprqtr/pdf/21c fr165.110.pdf. January 08, 2008.

FDA. 2006. Everything added to food in the United States. Food and Drug Administration. http://vm.cfsan.fda.gov/ dms/eafus.html. April 11, 2006.

FEDRIP. 2006. Phenol. Federal Research in Progress database. Springfield, VA: National Technical Information Service.

Fielding M, Gibson TM, McLoughlin K, et al. 1981. Organic micropollutants in drinking water. TR159. Medmenham, England. Water Research Center.

Flickinger CW. 1976. The benzenediols: Catechol, resorcinol and hydroquinone. A review of the industrial toxicology and current industrial exposure limits. Am Ind Hyg Assoc J 37:596-606.

Florin I, Rutberg L, Curvall M, et al. 1980. Screening of tobacco smoke constituents for mutagenicity using the Ames' test. Toxicology 18:219-232.

Fomon SJ. 1966. Body composition of the infant. Part I: The male reference infant. In: Falkner F, ed. Human development. Philadelphia, PA: WB Saunders, 239-246.

Fomon SJ, Haschke F, Ziegler EE, et al. 1982. Body composition of reference children from birth to age 10 years. Am J Clin Nutr 35:1169-1175.

*Forum for Scientific Excellence, Inc. 1990. Index of hazardous contents of commercial products in schools and colleges. Philadelphia, PA: JB Lippincott Co., 97, 109, 110, 145, 179.

Foxall PJD, Bending MR, Gartland KI, et al. 1989. Acute renal failure following accidental cutaneous absorption of phenol: Application of NMR urinalysis to monitor the disease process. Human Toxicol 9:491-496.

Freitag D, Lay JP, Korte F. 1984. Environmental hazard - Test results as related to structures and transplantation into the environment. In: Kaiser, KLE, ed. Quantitative Structure Activity Relationships Environmental Toxicology Proceedings Workshop. Boston, MA: D. Reidel Publishing Co., 111-136.

French MR, Bababunmi EA, Golding RR et al. 1974. The conjugation of phenol, benzoic acid, 1naphthylacetic acid and sulfadimethoxine in the lion, civet, and genet. FEBS Lett 46:134-137.

Fuhro R, Fromm D. 1978. Effects of compounds chemically related to salicylate on isolated antral mucosa of rabbits. Gastroenterology 75:661-667.

Gaffney JS, Streit GE, Spall WD, et al. 1987. Beyond acid rain: Do soluble oxidants and organic toxins interact with SO2 and NOX to increase ecosystem effects? Environ Sci Technol 21:519-524.

Gardner W, Cooke EI, Cooke RWI. 1978. Handbook of chemical synonyms and trade names. Boca Raton, FL: CRC Press, 150, 554. 
Ghantous H, Danielsson BR. 1986. Placental transfer and distribution of toluene, xylene and benzene, and their metabolites during gestation in mice. Biol Res Pregnancy Perinatol 7:98-105.

Gingell R, O’Donoghue JO, Staab RJ, et al. 2001. Phenol and phenolics. In: Bingham E, Cohrssen B, Powell Ch, eds. Patty's toxicology. $5^{\text {th }}$ ed. New York, NY: John Wiley \& Sons, 383-398.

Giwerceman A, Carlsen E, Keiding N, et al. 1993. Evidence for increasing incidence of abnormalities of the human testis: A review. Environ Health Perspect Suppl 101(2):65-71.

Gocke E, King MT, Eckhardt K, et al. 1981. Mutagenicity of cosmetics ingredients licensed by the European communities. Mutat Res 90:91-109.

Goerlitz DF, Troutman DE, Gody EM, et al. 1985. Migration of wood-preserving chemical in contaminated groundwater in a sand aquifer at Pensacola, Florida. Environ Sci Technol 19:955-961.

Goldfrank LR, Flomenbaum NE, Lewin NA, et al. 2002. Phenol. In: Goldfrank's toxicologic emergencies. 7th ed. New York, NY: McGraw-Hill, 1283-1284.

Goncharuk VV, Milyukin MV. 1999. Evaluation of contamination level of Dnieper River Basin by organic and inorganic toxicants. In: Baveye, PH, Block JC, Goncharuk VV eds. Bioavailability of organic xenobiotics in the environment. Netherlands: Kluwer Academic Publishers, 35-56.

Gossett RW, Brown DA, Young DR. 1983. Predicting the bioaccumulation of organic compounds in marine organisms using octanol/water partition coefficients. Marine Pollut Bull 14:387-392.

*Gould JP, Weber WJ, Jr. 1976. Oxidation of phenols by ozone. J Water Pollut Control Fed 48:47-60.

Graedel TE. 1978. Chemical compounds in the atmosphere. New York, NY Academic Press, 256.

Griffiths GJ. 1973. Fatal acute poisoning by intradermal absorption of phenol. Med Sci Law 13:46-48.

Gross BG. 1984. Cardiac arrhythmias during phenol face peeling. Plastic Reconst Surg 73:590-594.

Guerin MR, Jenkins RA, Tomkins BA. 1992. The chemical nature of environmental tobacco smoke: Composition and measurement. Chelsea, MI: Lewis Publishers, Inc., 24, 27, 39.

Gut I, Nedelcheva V, Soucek P, et al. 1996. Cytochromes P450 in benzene metabolism and involvement of their metabolites and reactive oxygen species in toxicity. Environ Health Perspect 104:1211-1218.

Guzelian PS, Henry CJ, Olin SS. 1992. Similarities and differences between children and adults:

Implications for risk assessment. Washington, DC: International Life Sciences Institute Press, 1-285.

Haddad LM, Shannon MW, Winchester JF. 1998. Phenol and related agents. In: Clinical management of poisoning and drug overdose. 3rd ed. Philadelphia, PA: WB Saunders Company, 956-960.

Haider K, Jagnow G, Kohnen R, et al. 1974. [Degradation of chlorinated benzol, phenol and cyclohexane derivatives by soil bacteria that utilize benzol and phenol under aerobic conditions.] Arch Microbiol 96:183-200. (German) 
Handson PD, Hanrahan PD. 1983. A rapid gas chromatographic method for the determination of free phenol in blood. J Agric Food Chem 31:447-448.

Hawley GG. 1981. The condensed chemical dictionary. 10th ed. New York, NY: Van Nostrand Reinhold Co, 796.

Haworth S, Lawler T, Mortelmans K, et al. 1983. Salmonella mutagenicity test results for 250 chemicals. Environ Mutagen Suppl 1:3-142.

Hawthorne SB, Sievers RE. 1984. Emission of organic air pollutants from shale oil wastewaters. Environ Sci Technol 18:483-490.

HazDat. 2006. Phenol. HazDat Database: ATSDR's Hazardous Substance Release and Health Effects Database. Atlanta, GA: Agency for Toxic Substances and Disease Registry. http://www.atsdr.cdc.gov/hazdat.html. April 06, 2006.

Healy JB, Young LY. 1978. Catechol and phenol degradation by a methanogenic population of bacteria. Food Microbiol Toxicol 35:216-218.

Heaton GD, Renwick AG. 1991. The effects of high dietary concentrations of sodium saccharin on in vivo metabolism of xenobiotics in rats. Food Chem Toxicol 29(5):305-312.

Heikkila PR, Hameila M, Pyy L, et al. 1987. Exposure to creosote in the impregnation and handling of impregnated wood. Scand J Work Environ Health 13:431-437.

Heller VG, Pursell L. 1938. Phenol-contaminated waters and their physiological action. J Pharmacol Exp Ther 63:99-107.

Hendrickson HP, Sahafayan M, Bell MA, et al. 1994. Relationship of flavonoid oxidation potential and effect on rat hepatic microsomal metabolism of benzene and phenol. J Pharm Biomed Anal 12:335-341.

Henschler R, Glatt HR. 1995. Induction of cytochrome P4501A1 in haemopoietic stem cells by hydroxylated metabolites of benzene. Toxicol In Vitro 9:453-457.

Hernberg S, Kurppa K, Ojajārvi J, et al. 1983. Congenital malformations and occupational exposure to disinfectants: A case-referent study. Scan J Work Environ Health 9:55.

Hinkel VGK, Kintzel HW. 1968. Phenolvergiftungen bei neugeborenen durch kutane resorption. Dtsch Gesundheitsw 23:2420-2422.

Hiser MF, Kropscott BE, McGuirk RJ, et al. 1994. Pharmacokinetics, metabolism and distribution of 14C-Phenol in Fischer 344 rats after oral gavage, drinking water and inhalation exposure. Dow Chemical Company. Submitted to U.S. Environmental Protection Agency under TSCA Section 8D. Study ID: K002727-022. OTS0557473.

Ho C, Lee KN, Jin QZ. 1983. Isolation and identification of volatile flavor compounds in fried bacon. J Agric Food Chem 31:336-342.

Hoel DG, Davis DL, Miller AB, et al. 1992. Trends in cancer mortality in 15 industrialized countries, 1969-1986. J Natl Cancer Inst 84(5):313-320. 
Hoff RM, Chan K. 1987. Measurement of polycyclic aromatic hydrocarbons in the air along the Niagara River. Environ Sci Technol 21:556-561.

Hoffman GM, Dunn BJ, Morris CR, et al. 2001. Two-week (ten-day) inhalation toxicity and two-week recovery study of phenol vapor in the rat. Int J Toxicol 20:45-52.

Hoffmann MJ, Ji S, Hedli CC, et al. 1999. Metabolism of $\left[{ }^{14} \mathrm{C}\right]$ phenol in isolated perfused mouse liver. Toxicol Sci 49:40-47.

Holland AJ, Youssef M. 1978. A complication of subarachnoid phenol blockade. Anaesthesia 34:260262.

Horch R, Spilker G, Stark GB. 1994. Phenol burns and intoxications. Burns 20:45-50.

Hoshika Y, Nihei Y, Muto G. 1981. Pattern display for characterization of trace amounts of odorants discharged from nine odor sources. Analyst (London) 106:1187-1202.

Hotchkiss SAM, Hewitt P, Caldwell J. 1992. Percutaneous absorption of nicotinic acid, phenol, benzoic acid and triclopyr butoxyethyl ester through rat and human skin in vitro: Further validation of an in vitro model by comparison with in vivo data. Food Chem Toxicol 30:891-899.

HSDB. 2008. Phenol. Hazardous Substances Data Bank. National Library of Medicine. http://toxnet.nlm.nih.gov. July 31, 2008.

Hsieh GC, Sharma RP, Parker RDR, et al. 1992. Immunological and neurobiochemical alterations induced by repeated oral exposure of phenol in mice. Eur J Pharmacol 228:107-114.

Hu JY, Aizawa T. 2003. Quantitative structure-activity relationships for estrogen receptor binding affinity of phenolic chemicals. Water Res 37(6):1213-1222.

Huang Z, Zhou W, Zhou C, et al. 2003. Determination of semivolatile compounds in drinking water by tandem mass spectrometric detection. Bull Environ Contam Toxicol 71(5):1026-1033.

Hughes MF, Hall LL. 1995. Disposition of phenol in rat after oral, dermal, intravenous, and intratracheal administration. Xenobiotica 25:873-883.

Humphrey MJ, Filer CW, Jeffery DJ, et al. 1980. The availability of carfecillin and its phenol moiety in rat and dog. Xenobiotica 10:771-778.

Hunter DM, Timerding BL, Leonard RB, et al. 1992. Effects of isopropyl alcohol, ethanol, and polyethylene glycol/industrial methylated spirits in the treatment of acute phenol burns. Ann Emerg Med 21:1303-1307.

IARC. 1986. IARC Monographs on the evaluation of the carcinogenic risk of chemicals to humans. Vol. 38. Tobacco smoking. Lyon, France: World Health Organization, International Agency for Research on Cancer, 104-105. 


\section{REFERENCES}

IARC. 1989. Phenol In: IARC Monographs on the evaluation of carcinogenic risk of chemicals to humans, Vol 47. Some organic solvents, resin monomers and related compounds, pigments and occupational exposures in paint manufacturing and painting. Lyon, France: World Health Organization, International Agency for Research on Cancer, 263-287.

IARC. 2004. Overall evaluations of carcinogenicity to humans: As evaluated in IARC Monographs volumes 1-82 (at total of 900 agents, mixtures and exposures). Lyon, France: International Agency for Research on Cancer. http://www.cie.iarc.fr/monoeval/crthall.html. March 08, 2006.

Inoue $\mathrm{H}$, Yokota $\mathrm{H}$, Taniyama $\mathrm{H}$, et al. 1999. 1-Naphthol $\beta$-D-glucuronide formed intraluminaly in rat small intestine mucosa and absorbed into the colon. Life Sci 65(15):1579-1588.

IRIS. 2006. Phenol. Washington, DC: Integrated Risk Information System. U.S. Environmental Protection Agency. http://www.epa.gov/iris/subst/index.html. March 8, 2006.

Itoh M. 1995. The role of brain acetylcholine in phenol-induced tremor in mice. Arch Oral Biol 40:365372.

Iwasaki K, Shiraga T, Takeshita K, et al. 1993. Perinatal development of amine, alcohol and phenol sulfoconjugations in the rat. Res Commun Chem Pathol Pharmacol 81:183-190.

Jansson T, Curvall M, Hedin A, et al. 1986. In vitro studies of biological effects of cigarette smoke condensate. II. Induction of sister chromatid exchanges in human lymphocytes by weakly acidic, semivolatile constituents. Mutat Res 169:129-139.

Jarvis SN, Straube RC, Williams ALJ, et al. 1985. Illness associated with contamination of drinking water supplies with phenol. Br Med J 290:1800-1802.

Jay K, Stieglitz L. 1995. Identification and quantification of volatile organic components in emissions of waste incineration plants. Chemosphere 30:1249-1260.

Johansen SS, Hansen AB, Mosboek H, et al. 1997. Identification of heteroaromatic and other organic compounds in ground water at creosote-contaminated sites in Denmark. Ground Water Monit Remed 7:106-115.

Johanson CE. 1980. Permeability and vascularity of the developing brain: Cerebellum vs. cerebral cortex. Brain Res 190:3-16.

Jungclaus GA, Lopez-Avila V, Hites RA. 1978. Organic compounds in an industrial waste water: A case study of their environmental impact. Environ Sci Technol 12:88-96.

Kaiser HE. 1967. Cancer-promoting effects of phenols in tea. Cancer 20:614-616.

Kamijo Y, Soma K, Fukuda M, et al. 1999. Rabbit syndrome following phenol ingestion. Clin Toxicol 37:509-511.

Kao J, Bridges JW, Faulkner JK. 1979. Metabolism of $\left[{ }^{14} \mathrm{C}\right]$ phenol by sheep, pig, rat. Xenobiotica 9:141-147.

Kauppinen TP, Partanen TJ, Nurminen MM. 1986. Respiratory cancers and chemical exposures in the wood industry: A nested case-control study. Br J Ind Med 43:84-90. 
Kavlock RJ. 1990. Structure-activity relationships in the developmental toxicity of substituted phenols: In vivo effects. Teratology 41:43-59.

Kawamoto T, Koga M, Oyama T, et al. 1996. Habitual and genetic factors that affect urinary background levels of biomarkers for organic solvent exposure. Arch Environ Contam Toxicol 30:114120.

Keith LH. 1976. Identification of organic compounds in unbleached treated Kraft paper mill wastewaters. Environ Sci Technol 10:555-564.

Kenyon EM, Seeley ME, Janszen D, et al. 1995. Dose-, route-, and sex-dependent urinary excretion of phenol metabolites in $\mathrm{B}_{6} \mathrm{C}_{3} \mathrm{~F}_{1}$ mice. J Toxicol Environ Health 44:219-233.

Kim D-H, Lee S-K, Chun B-Y, et al. 1994. Illness associated with contamination of drinking water supplies with phenol. J Korean Med Sci 9:218-223.

Kim HJ, Cho JH, Klaassen CD. 1995. Depletion of hepatic 3'-phosphoadenosine 5'-phosphosulfate (PAPS) and sulfate in rats by xenobiotics that are sulfated. J Pharmacol Exp Ther 275:654-658.

Kincannon DF, Stover EL, Nichols V, et al. 1983. Removal mechanisms for toxic priority pollutants. J Water Pollut Control Fed 55:157-163.

Kobayashi K, Akitake H, Manadge K. 1979. Relation between toxicity and accumulation of various chlorophenols in goldfish. Bull Jap Soc Sci Fish 45:173-175.

Kolachana P, Subrahmanyam VV, Meyer KB, et al. 1993. Benzene and its phenolic metabolites produce oxidative DNA damage in HL60 cells in vitro and in the bone marrow in vivo. Cancer Res 53(5):10231026.

Kolpin DW, Furlong ET, Meyer MT, et al. 2002. Pharmaceuticals, hormones, and other organic wastewater contaminants in US streams, 1999-2000: A national reconnaissance. Environ Sci Technol 36:1202-1211.

Komori M, Nishio K, Kitada M, et al. 1990. Fetus-specific expression of a form of cytochrome P-450 in human liver. Biochemistry 29:4430-4433.

Konasewich D, Traversy W, Zar H. 1978. Status report on organic and heavy metal contaminants in the Lake Erie, Michigan, Huron and Superior Basins. Windsor, Ontario: International Joint Commission Great Lakes Water Quality Board, 161.

Koop DR, Laethem CL, Schnier GG. 1989. Identification of ethanol-inducible P450 isozyme 3a (P450IIE1) as a benzene and phenol hydroxylase. Toxicol Appl Pharmacol 98:278-288.

Koster H, Halsema I, Scholtens E, et al. 1981. Dose-dependent shifts in the sulfation and glucuronidation of phenolic compounds in the rat in vivo and in isolated hepatocytes. The role of saturation of phenolsulfotransferase. Biochem Pharmacol 30:2569-2575.

Kostiainen R. 1995. Volatile organic compounds in the indoor air of normal and sick houses. Atmos Environ 29:693-702. 
Kothare PA, Zimmerman CL. 2002. Intestinal metabolism: The role of enzyme localization in phenol metabolite kinetics. Drug Metab Dispos 30(5):586-594.

Kot-Wasik A, Kartanowicz R, Dabrowski D, et al. 2004. Determination of chlorophenols and phenoxyacid herbicides in the Gulf of Gdansk, southern Baltic Sea. Bull Environ Contam Toxicol 73:511-518.

Krishnan K, Andersen ME. 1994. Physiologically-based pharmacokinetic modeling in toxicology. In: Hayes W, ed. Principles and methods of toxicology. 3rd ed. New York, NY: Raven Press, Ltd.

Krishnan K, Andersen ME, Clewell HJ, III, et al. 1994. Physiologically-based pharmacokinetic modeling of chemical mixtures. In: Yang RSA, ed. Toxicology of chemical mixtures. New York, NY: Academic Press, 399-437.

Kubo T, Urano K, Utsumi H. 2002. Mutagenicity characteristics of 255 environmental chemicals. J Health Sci 48(6):545-554.

Kuwata K, Uebori M, Yamazaki Y. 1980. Determination of phenol in polluted air as pnitrobenzeneazophenol derivative by reversed phase high performance liquid chromatography. Anal Chem 52:857-860.

Langford CP, Bartlett R, Haddad LM. 1998. Phenol and related agents. In: Haddad LM, Shannon MW, Winchester JF, eds. Clinical management of poisoning and drug overdose. Philadelphia, PA: Saunders, 956-960.

Lebedev AT, Poliakova OV, Karakhanova NK, et al. 1998. The contamination of birds with organic pollutants in the Lake Baikal region. Sci Total Environ 212:153-162.

Leeder JS, Kearns GL. 1997. Pharmacogenetics in pediatrics: Implications for practice. Ped Clin North America 44:55-77.

Legathe A, Hoener B-A, Tozer TN. 1994. Pharmacokinetic interaction between benzene metabolites, phenol and hydroquinone, in $\mathrm{B} \mathrm{C}_{3} \mathrm{~F}_{1}$ mice. Toxicol Appl Pharmacol 124:131-138.

Leuenberger C, Ligocki MP, Pankow JF. 1985. Trace organic compounds in rain. 4. Identities, concentrations and scavenging mechanisms for phenols in urban air and rain. Environ Sci Technol 19:1053-1058.

Leung H. 1993. Physiologically-based pharmacokinetic modeling. In: Ballantyne B, Marrs T, Turner P, eds. General and applied toxicology. Vol. I. New York, NY: Stockton Press, 153-164.

Lewin JF, Cleary WT. 1982. An accidental death caused by the absorption of phenol through skin. A case report. Forensic Sci Int 19:177-179.

Lewis RJ. 2000. Sax's dangerous properties of industrial materials. 10th ed. New York, NY: John Wiley \& Sons, Inc., 2884-2885.

Lewis RJ. 2001. Hawley's condensed chemical dictionary. 14th ed. New York, NY: John Wiley \& Sons, Inc., 857. 
Li Y, Qu M, Sun L, et al. 2005. Genotoxicity study of phenol and o-cresol using the micronucleus test and the comet assay. Toxicol Environ Chem 87(1-4):365-372.

Liao TF, Oehme FW. 1981. Tissue distribution and plasma protein binding of $\left[{ }^{14} \mathrm{C}\right]$ phenol in rats. Toxicol Appl Pharmacol 57:220-225.

Lide DR. 2005. CRC handbook of chemistry and physics. Boca Raton, FL: CRC Press, 3-422.

Lide DR, Milne GWA. 1994. Phenol. In: Handbook of data on organic compounds. Boca Raton, FL: CRC Press, 3990.

Ling WH, Hanninen O. 1991. Shifting from a conventional diet to an uncooked vegan diet reversibly alters fecal hydrolytic activities in humans. J Nutr 122:924-930.

Livingston AL. 1978. Forage plant estrogens. J Toxicol Environ Health 4:301-324.

Lo Dico C, Caplan YH, Levine B, et al. 1989. Phenol: Tissue distribution in a fatality. J Forensic Sci 34:1013-1015.

Lopes TJ, Furlong ET. 2001. Occurrence and potential adverse effects of semivolatile organic compounds in streambed sediment, United States, 1992-1995. Environ Sci Technol 20:727-737.

Lopez-Avila V, Northcutt R, Onstot J. et al. 1983. Determination of 51 priority organic compounds after extraction from standard reference materials. Anal Chem 55:881-889.

Ludzack FJ, Ettinger MB. 1960. Chemical structures resistant to aerobic biochemical stabilization. J Water Pollut Control Fed 32:1173-1200.

Lüttke J, Levsen K, Acker K, et al. 1999. Phenols and nitrated phenols in clouds at Mount Brocken. Int J Environ Anal Chem 74:69-89.

Lunte SM, Kissinger PT. 1983. Detection and identification of sulfhydryl conjugates of p-benzoquinone in microsomal incubations of benzene and phenol. Chem-Biol Interact 47:195-212.

Lyman WJ, Reehl WF, Rosenblatt DH. 1982. Handbook of chemical property estimation methods. Environmental behavior of organic compounds. New York, NY: McGraw-Hill Book Co., 15-9 to 15-31.

Mayr U, Butsch A, Schneider S. 1992. Validation of two in vitro test systems for estrogenic activities with zearalenone, phytoestrogens and cereal extracts. Toxicology 74:135-149.

McDonald JD, Zielinska B, Jujita EM, et al. 2000. Fine particle and gaseous emission rates from residential wood combustion. Environ Sci Technol 34:2080-2091.

McFadden SA. 1996. Phenotypic variation in xenobiotic metabolism and adverse environmental response: Focus on sulfur-dependent detoxification pathways. Toxicology 111:43-65.

*Medinsky MA. 1995. The application of physiologically based pharmacokinetic/pharmacodynamic (PBPK/PD) modeling to understanding the mechanism of action of hazardous substances. Toxicol Lett 79:185-191. 


\section{REFERENCES}

Medinsky MA, Kenyon EM, Schlosser PM. 1995. Benzene: A case study in parent chemical and metabolite interactions. Toxicology 105:225-233.

Mehta R, Hirom PC, Millburn P. 1978. The influence of dose on the pattern of conjugation of phenol and 1-naphthol in nonhuman primates. Xenobiotica 8:445-452.

Merliss RR. 1972. Phenol marasmus. Occup Med 14:55-56.

Migaszewski ZM. 1999. Determining organic compound ratios in soils and vegetation of the Holy Cross Mountains, Poland. Water Air Soil Pollut 111(1-4):123-138.

Mill T, Mabey W. 1985. Photochemical transformations. In: Neely WB, Blau GE, eds. Environmental exposure from chemicals. Vol. 1. Boca Raton, FL: CRC Press, 175-216.

Miller BM, Pujadas E, Gocke E. 1995. Evaluation of the micronucleus test in vitro using Chinese hamster cells: Results of four chemicals weakly positive in the in vivo micronucleus test. Environ Mol Mutagen 26:240-247.

Miller JJ, Powell GM, Olavesen AH, et al. 1976. The toxicity of dimethoxyphenol and related compounds in the cat. Toxicol Appl Pharmacol 3:47-57.

Miyagawa M, Takasawa H, Sugiyama A, et al. 1995. The in vivo-in vitro replicative DNA synthesis (RDS) test with hepatocytes prepared from male $\mathrm{B} 6 \mathrm{C}_{3} \mathrm{~F}_{1}$ mice as an early prediction assay for putative nongenotoxic (Ames-negative) mouse hepatocarcinogens. Mutat Res 343:157-183.

Monteiro-Riviere NA, Inmann AO, Jackson H, et al. 2001. Efficacy of topical phenol decontamination strategies on severity of acute phenol chemical burns and dermal absorption: In vitro and in vivo studies. Toxicol. Ind. Health 17(4):95-104.

Moreira dos Santos CY, de Almeida Azevedo D, Radler de Aquino Neto F. 2004. Atmospheric distribution of organic compounds from urban areas near a coal-fired power station. Atmos Environ 38(9):1247-1257.

* Morgan RJ, Steller PH. 1994. Acute paraplegia following intrathecal phenol block in the presence of occult epidural malignancy. Anaesthesia 49:142-144.

Morimoto K, Wolff S. 1980. Increase of sister chromatid exchanges and perturbations of cell division kinetics in human lymphocytes by benzene metabolites. Cancer Res 40:118-1193.

Morimoto K, Wolff S, Koizumi A. 1983. Induction of sister-chromatid exchanges in human lymphocytes by microsomal activation of benzene metabolites. Mutat Res 119:355-360.

Morrison JE, Matthews D, Washington R, et al. 1991. Phenol motor point blocks in children: Plasma concentrations and cardiac dysrhythmias. Anesthesiology 75:359-362.

Morselli PL, Franco-Morselli R, Bossi L. 1980. Clinical pharmacokinetics in newborns and infants. Clin Pharmacokin 5:485-527.

Moser VC, Cheek BM, MacPhail RC. 1995. A multidisciplinary approach to toxicological screening: III. Neurobehavioral toxicity. J Toxicol Environ Health 45:173-210. 
Mulder GJ, Scholtens E. 1977. Phenol sulphotransferase and uridine diphosphate glucuronyltransferase from rat liver in vivo and in vitro. Biochem J 165:553-559.

Muraleedharan TR, Radojevic M, Waugh A, et al. 2000. Chemical characterization of the haze in Brunei Darussalam during the 1998 episode. Atmos Environ 34(17):2725-2731.

Murphy JC, Osterberg RE, Seabaugh VM, et al. 1982. Ocular irritancy responses to various pHs of acids and bases with and without irrigation. Toxicology 23:281-291.

Murray AR, Kisin E, Castranova V, et al. 2007. Phenol-induced in vivo oxidative stress in skin: Evidence for enhanced free radical generation, thiol oxidation, and antioxidant depletion. Chem Res Toxicol 20(12):1769-1777.

*Musto JD, Sane JN, Warner VD. 1977. Quantitative determination of phenol by high-pressure liquid chromatography. J Pharm Sci 66:1201-1202.

Nagel R, Adler HI, Rao TK. 1982. Induction of filamentation by mutagens and carcinogens in a ionmutant of Escherichia coli. Mutat Res 105:309-312.

*Nanbo T. 1993. Influence of dietary lipids on glucuronidation and sulfation of phenol and its para substituent in the rat. Biol Pharm Bull 16:518-520.

NARA. 2006. Phenol. Electronic Code of Federal Regulations. Washington, DC: National Archives and Records Administration. http://www.access.gpo.gov/nara/cfr/cfr-table-search.html. March 20, 2006.

Narotsky MG, Kavlock RJ. 1995. A multidisciplinary approach to toxicological screening: II. Developmental toxicity. J Toxicol Environ Health 45:145-171.

NAS/NRC. 1989. Biologic markers in reproductive toxicology. National Academy of Sciences/National Research Council. Washington, DC: National Academy Press, 15-35.

Nazaroff WM, Singer BC. 2004. Inhalation of hazardous air pollutants from environmental tobacco smoke in U.S. residences. J Expo Anal Environ Epidemiol 14:871-877.

NCI. 1980. Bioassay of phenol for possible carcinogenicity. Bethesda, MD: U.S. Department of Health and Human Services. National Cancer Institute. NCI-CG-TR-203.

NCI. 1998. Cigars. Health effects and trends. Smoking and tobacco control monograph 9. Bethesda, MD: U.S. Department of Health and Human Services. National Cancer Institute. NIH Pub. No. 984302.

Needham LL, Head SL, Cline RE. 1984. Determination of phenols and cresols in urine by gas chromatography. Anal Lett 17:1555-1565.

Nicola RM, Branchflower R, Pierce D. 1987. Chemical contaminants in bottomfish. J Environ Health 49:342-347.

Nieminen E, Heikkila P. 1986. Simultaneous determination of phenol, cresols and xylenols in workplace air, using a polystyrene-divinylbenzene column and electrochemical detection. J Chromatogr 360:271278. 
Nilsson A, Lagesson V, Bornehag C-G, et al. 2005. Quantitative determination of volatile organic compounds in indoor dust using gas chromatography-UV spectrometry. Environ Int 31:1141-1148.

NIOSH. 1983. Health hazard evaluation report No. HETA 82-053-1263. Bay Area Hospital, Coos Bay, Oregon. Cincinnati, OH: National Institute for Occupational Safety and Health. PB84210525.

NIOSH. 1990. Phenol. National occupational exposure survey 1981-1983. Cincinnati, OH: U.S. Department of Health and Human Services, Public Health Service, Centers for Disease Control. National Institute for Occupational Safety and Health. http://www.cdc.gov/noes/noes1/55460sic.html. July 31, 2008.

NIOSH. 1994b. Method 2546. Cresol (all isomers) and phenol. NIOSH manual of analytical methods. 4th ed. National Institute of Occupational Safety and Health. (NMAM). 4th ed. http://www.cdc.gov/niosh/nmam/pdfs/2546.pdf. July 31, 2008.

NIOSH. 1994a. Method 8305: Phenol and p-cresol in urine. NIOSH manual of analytical methods (NMAM). 4th ed. National Institute of Occupational Safety and Health. http://www.cdc.gov/niosh/nmam/pdfs/8305.pdf. May 25, 2006.

NIOSH. 1996. Method 2549. Volatile organics compounds (screening) NIOSH manual for analytical methods(NMAM). 4th ed. National Institute for Occupational Safety and Health. http://www.cdc.gov/niosh/nmam/pdfs/2549.pdf. July 31, 2008.

NIOSH. 2005. Phenol. NIOSH pocket guide to chemical hazards. Atlanta, GA: National Institute for Occupational Safety and Health, Centers for Disease Control and Prevention. http://www.cdc.gov/niosh/npg/. March 08, 2006.

Nishihara T, Nishikawa J, Kanayama T, et al. 2000. Estrogenic activities of 517 chemicals by yeast twohybrid assay. J Health Sci.46(4):282-298.

Nomoto Y, Fujita T, Kitani Y. 1987. Serum and urine levels of phenol following phenol blocks. Can J Anaesth 34(3):307-310.

NRC. 1993. Pesticides in the diets of infants and children. National Research Council, Washington DC: National Academy Press.

NTP. 1983a. Teratologic evaluation of phenol (CAS No. 108-95-2) in CD-1 rats. National Toxicology Program. Research Triangle Park, NC: Research Triangle Institute.

NTP. 1983b. Teratologic evaluation of phenol (CAS No. 108-95-2) in CD mice. Laboratory study: September 18, 1980 to January 12, 1981. National Toxicology Program. Research Triangle Park, NC: Research Triangle Institute.

NTP. 2005. Report on carcinogens. 11th ed. Research Triangle Park, NC: National Toxicology Program, Department of Health and Human Services. http://ntp-server.niehs.nih.gov/ntp/roc/toc11.html. January 11, 2008.

O'Grodnick JS, Dupre GD, Gulizia BJ, et al. 1983. Determination of benzene, phenol, catechol and hydroquinone in whole blood of rats and mice. J Chromatog Sci 21:289-292. 


\section{REFERENCES}

Ohtsuji H, Ikeda M. 1972. Quantitative relation between atmospheric phenol vapor and phenol in the urine of workers in Bakelite factories. Br J Ind Med 29:70-73.

O’Neil MJ. 2001. Phenol. In: Merck index: An encyclopedia of chemicals, drugs, and biologicals. Whitehouse Station, NJ: Merck Research Laboratories, 1299-1300.

OSHA. 2005a. Air contaminants. Occupational safety and health standards for shipyard employment. Washington, DC: Occupational Safety and Health Administration. Code of Federal Regulations. 29 CFR 1915.1000. http://www.osha.gov/comp-links.html. March 08, 2006.

OSHA. 2005b. Gases, vapors, fumes, dusts, and mists. Safety and health regulations for construction. Washington, DC: Occupational Safety and Health Administration. Code of Federal Regulations. 29 CFR 1926.55, Appendix A. http://www.osha.gov/comp-links.html. March 08, 2006.

OSHA. 2005c. Limits for air contaminants. Occupational safety and health standards. Washington, DC: Occupational Safety and Health Administration. Code of Federal Regulations. 29 CFR 1910.1000. http://www.osha.gov/comp-links.html. March 06, 2006.

OTA. 1990. Neurotoxicology: Identifying and controlling poisons of the nervous system. Washington, DC: Office of Technology Assessment. OTA-BA-438.

*OTII. 2006. Phenol imports and exports. Washington, DC: U.S. Department of Commerce, Office of Trade and Industry Information, International Trade Administration. http://www.ita.doc.gov/td/industry/otea/trade-detail/index.html. July 14, 2006.

Owen GM, Brozek J. 1966. Influence of age, sex, and nutrition on body composition during childhood and adolescence. In: Falkner, ed. Human development. Philadelphia, PA: Saunders, 222-238.

Painter RB, Howard R. 1982. The HeLa DNA-synthesis inhibition test as a rapid screen for mutagenic carcinogens. Mutat Res 92:427-437.

Parkhurst BR, Bradshaw AS, Forte JL, et al. 1979. An evaluation of the acute toxicity to aquatic biota of a coal conversion effluent and its major components. Bull Environ Contam Toxicol 23:349-356.

Paschin YV, Bahitova LM. 1982. Mutagenicity of benzo[a]pyrene and the antioxidant phenol at the HGPRT locus of V79 Chinese hamster cells. Mutat Res 104:389-393.

Pashin YV, Bakhitova LM, Bentkhen TI. 1987. Dependence of antimutagenic activity of simple phenols on the number of hydroxyl groups. Bull Exp Biol Med 102(2):1121-1123.

Paterson B, Cowie CE, Jackson PE. 1996. Determination of phenols in environmental waters using liquid chromatography with electrochemical detection. J Chromatogr 731:95-102.

Patrick E, Maibach HI, Burkhalter A. 1985. Mechanisms of chemically induced skin irritation. I. Studies of time course, dose response, and components of inflammation in the laboratory mouse. Toxicol Appl Pharmacol 81:476-490.

PDR. 2005. Physicians' desk reference. Montvale, NJ: Thomson PDR, 2693.

Pellack-Walker P, Blumer JL. 1986. DNA damage in L5178YS cells following exposure to benzene metabolites. Mol Pharmacol 39:42-47. 


\section{REFERENCES}

Perkins MA, Osborne R, Johnson GR. 1996. Development of an in vitro method for skin corrosion testing. Fundam Appl Toxicol 31:9-18.

Petrasek AC, Kugelman IJ, Austern BM, et al. 1983. Fate of toxic organic compounds in wastewater treatment plants. J Water Pollut Control Fed 55:1286-1296.

Pfeffer FM. 1979. The 1977 screening survey for measurement of organic priority pollutants in petroleum refinery wastewaters. ASTM Spec Tech Publ 686:181-190.

Pierrehumbert G, Pierre-Oliver D, Tardif R, et al. 2002. Impact of human variability on the biological monitoring of exposure to toluene, phenol, lead, and mercury: II. Compartmental based toxicokinetic modelling. Toxicol Lett 134:165-175.

Piotrowski JK. 1971. Evaluation of exposure to phenol: Absorption of phenol vapor in the lungs through the skin and excretion of phenol in urine. Br J Ind Med 28:172-178.

Plumb RH Jr. 1987. A comparison of ground water monitoring data from CERCLA and RCRA sites. Ground Water Monit Rev 7:94-100.

Poirier MC, DeCicco BT, Lieberman MW. 1975. Nonspecific inhibition of DNA repair synthesis by tumor promotors in human diploid fibroblasts damage with $\mathrm{N}$-acetoxy-2-acetylaminofluorene. Cancer Res 35:1392-1397.

Poliakova OV, Lebedev AT, Hanninen O. 2000. Organic pollutants in snow of urban and rural Russia and Finland. Toxicol Environ Chem 75:181-194.

Pool PL, Lin PZ. 1982. Mutagenicity testing in the Salmonella typhimurium assay of phenolic compounds and phenolic fractions obtained from smokehouse smoke condensates. Food Chem Toxicol 29:383-391.

Popp W, Rauscher D, Muller G, et al. 1994. Concentrations of benzene in blood and Sphenylmercapturic acid and $t, t$-muconic acid in urine in car mechanics. Int Arch Occup Environ Health $66: 1-6$.

Post G, Snyder R, Kalf GF. 1986. Metabolism of benzene and phenol in macrophages in vitro and the inhibition of RNA synthesis by benzene metabolites. Cell Biol Toxicol 2:231-246.

Powell GM, Miller JJ, Olavesen AH, et al. 1974. Liver as major organ of phenol detoxification. Nature (London) 252:234-235.

Powley MW, Carlson GP. 2001. Cytochrome P450 isozymes involved in the metabolism of phenol, a benzene metabolite. Toxicol Lett 125:117-123.

Powlowski J, Shingler V. 1994. Genetics and biochemistry of phenol degradation by Pseudomonas sp. CF600. Biodegradation 5:219-236.

Pullin TG, Pinkerton MN, Johnson RV, et al. 1978. Decontamination of the skin of swine following phenol exposure: A comparison of the relative efficacy of water versus polyethylene glycol/industrial methylated spirits. Toxicol Appl Pharmacol 43:199-206. 
Quebbemann AJ, Anders MW. 1973. Renal tubular conjugation and excretion of phenol and pnitrophenol in the chicken: Differing mechanisms of renal transfer. J Pharmacol Exp Ther 184:695-708.

Quint U, Muller RT, Muller G. 1998. Characteristics of phenol: Instillation in intralesional tumor excision of shondroblastoma, osteoclastoma and enchondroma. Arch Orthop Trauma Surg 117:43-46.

Ramakrishna BS, Roberts-Thomson IC, Pannall PR, et al. 1991. Impaired sulphation of phenol by the colonic mucosa in quiescent and active ulcerative colitis. Gut 32:46-49.

Ramli JB, Wheldrake JF. 1981. Phenol conjugation in the desert hopping mouse, Notomys alexis. Comp Biochem Physiol 69C:379-381.

Reddy MV, Bleicher WT, Blackburn GR, et al. 1990. DNA adduction by phenol, hydroquinone, or benzoquinone in vitro but not in vivo: Nuclease P1-enhanced ${ }^{32} \mathrm{P}$-postlabeling of adducts as labeled nucleoside bisphosphates, dinucleotides and nucleoside monophosphates. Carcinogenesis 11:1349-1357.

Rick DL, Licht CF, McCarty LP. 1982. Determination of phenol and pentachlorophenol in plasma and urine samples by gas liquid chromatography. J Anal Toxicol 6:297-300.

Roemer E, Stabbert R, Rustemeier K, et al. 2004. Chemical composition, cytotoxicity and mutagenicity of smoke from U.S. commercial and reference cigarettes smoked under two sets of machine smoking conditions. Toxicology 195:31-52.

Rogers SCF, Burrows D, Neill D. 1978. Percutaneous absorption of phenol and methyl alcohol in magenta paint. Br J Dermatol 98:559-560.

Rothman N, Bechtold WE, Yin S-N, et al. 1998. Urinary excretion of phenol, catechol, hydroquinone, and muconic acid by workers occupationally exposed to benzene. Occup Environ Med 55:705-711.

Rubin HE, Alexander M. 1983. Effect of nutrients on the rates of mineralization of trace concentrations of phenol and p-nitrophenol. Environ Sci Technol 17:104-107.

Ryan BM, Selby R, Gingell R, et al. 2001. Two-generation reproduction study and immunotoxicity screen in rats dosed with phenol via the drinking water. Int J Toxicol 20:121-142.

Sabourin PJ, Bechtold WE, Griffith WC, et al. 1989. Effect of exposure concentration, exposure rate, and route of administration on metabolism of benzene by F344 rats and $\mathrm{B} 6 \mathrm{C} 3 \mathrm{~F} 1$ mice. Toxicol Appl Pharmacol 99:421-444.

Sabourin PJ, Chen BT, Lucier G, et al. 1987. Effect of dose on the absorption and excretion of $\left[{ }^{14} \mathrm{C}\right]$ benzene administered orally or by inhalation in rats and mice. Toxicol Appl Pharmacol 87:325-336.

Sacan MT, Balcioglu IA. 1996. Prediction of the soil sorption coefficient of organic pollutants by the characteristic root index model. Chemosphere 32(10):1993-2001.

Salaman MH, Glendenning OM. 1957. Tumor promotion in mouse skin by sclerosing agents. $\mathrm{Br} \mathrm{J}$ Cancer 11:434-444.

Sawahata T, Neal RA. 1983. Biotransformation of phenol to hydroquinone and catechol by rat liver microsomes. Mol Pharmacol 23:453-460. 
Schaltenbrand WE, Coburn SP. 1985. Determination of phenol and p-cresol in urine. Clin Chem 31:2042-2043.

Schauer JJ, Kleeman MJ, Cass GR, et al. 2001. Measurement of emissions from air pollution sources. 3. $\mathrm{C}_{1}-\mathrm{C}_{29}$ organic compounds from fireplace combustion of wood. Environ Sci Technol 35:1716-1728.

Schlosser PM, Bond JA, Medinsky MA. 1993. Benzene and phenol metabolism by mouse and rat liver microsomes. Carcinogenesis 14:2477-2486.

Schmidt SH, Hellström S. 1993. Phenol anesthesia of the tympanic membrane in purulent otitis media A structural analysis in the rat. Eur Arch Otorhinolaryngol 249:470-472.

Schmidt SH, Anniko M, Hellström S. 1990. Electrophysiological effects of the clinically used local anesthetics lidocaine, lidocaine-prilocaine and phenol on the rat's inner ear. Eur Arch Otorhinolaryngol 248:87-94.

Schwartz CS, Snyder R, Kalf GF. 1985. The inhibition of mitochondrial DNA replication in vitro by the metabolites of benzene, hydroquinone and p-benzoquinone. Chem-Biol Interact 53:327-350.

Scott DO, Lunte CE. 1993. In vivo microdialysis sampling in the bile, blood, and liver of rats to study the disposition of phenol. Pharmaceutical Res 10:335-342.

Scott HD, Wolf DC, Lavy TL. 1983. Adsorption and degradation of phenol at low concentrations in soil. J Environ Qual 12:91-95.

Scully FE, Jr, Hoigne JR. 1987. Rate constants for reactions of singlet oxygen with phenols and other compounds in water. Chemosphere 16:681-694.

Seaton MJ, Schlosser PM, Medinsky MA. 1995. In vitro conjugation of benzene metabolites by human liver: Potential influence of interindividual variability on benzene toxicity. Carcinogenesis 16:15191527.

Semple KT, Cain RB. 1996. Biodegradation of phenols by the alga Ochromonas danica. Appl Environ Microbiol 62:1265-1273.

Setchell BP, Waites GMH. 1975. The blood testis barrier. In: Creep RO, Astwood EB, Greiger SR, eds. Handbook of physiology: Endocrinology V. Washington, DC: American Physiological Society, 143172.

Shafer KH, Cooke M, Deroos F, et al. 1981. WCOT capillary GC/FT-IR and GC/MS for identifying toxic organic pollutants. Appl Spectrosc 35:469-472.

Shamy MY, El Gazzar RM, El Sayed MA, et al. 1994. Study of some biochemical changes among workers occupationally exposed to phenol, alone or in combination with other organic solvents. Ind Health 32:207-214.

Shelby MD, Witt KL. 1995. Comparison of results from mouse bone marrow chromosomes aberration and micronucleous tests. Environ Mol Mutagen 25(4):302-313.

Sheldon LS, Hites RA. 1978. Organic compounds in the Delaware River. Environ Sci Technol 12:11881194. 
Sheldon LS, Hites RA. 1979. Sources and movement of organic chemicals in the Delaware River. Environ Sci Technol 13:574-579.

Shiu WY, Ma KC, Varhanickova AD, et al. 1994. Chlorophenols and alkylphenols: A review and correlation of environmentally relevant properties and fate in an evaluative environment. Chemosphere 29:1155-1224.

Sithole BB, Williams DT, Lastoria C, et al. 1986. Determination of halogenated phenols in raw and potable water by selected ion gas chromatography-mass spectrometry. J Assoc Off Anal Chem 69:466473.

Skare JA, Schrotel KR. 1984. Alkaline elution of rat testicular DNA: Detection of DNA strand breaks after in vivo treatment with chemical mutagens. Mutat Res 130:283-294.

Skowronski GA, Kadry AM, Turkall RM, et al. 1994. Soil decreases the dermal penetration of phenol in male pig in vitro. J Toxicol Environ Health 41:467-479.

Smart RC, Zannoni VG. 1984. DT-Diaphorase and peroxidase influence the covalent binding of the metabolites of phenol, the major metabolite of benzene. Mol Pharmacol 26:105-111.

Snyder R, Chepiga T, Yang CS, et al. 1993. Benzene metabolism by reconstituted cytochromes P450 2B1 and 2E1 and its modulation by cytochrome b(5), microsomal epoxide hydrolase, and glutathione transferases: Evidence for an important role of microsomal epoxide hydrolase in the formation of hydroquinone. Toxicol Appl Pharmacol 122(2):172-181.

Soares ER, Tift JP. 1982. Phenol poisoning: Three fatal cases. J Forensic Sci 27(3):729-731.

Southworth GR, Keller JL. 1986. Hydrophobic sorption of polar organics by low organic carbon soils. Water Air Soil Pollut 28:239-248.

Spiller HA, Quandrani-Kushner DA, Cleveland P. 1993. A five year evaluation of acute exposures to phenol disinfectant (26\%). J Toxicol Clin Toxicol 31:307-313.

Sporns P. 1981. High pressure liquid chromatographic determination of phenol in honey. J Assoc Off Anal Chem 64:337-340.

SRI. 2006. Phenol. 2005 Directory of chemical producers. Menlo Park, CA: SRI Consulting, 785.

Stajduhar-Caric Z. 1968. Acute phenol poisoning. Singular findings in a lethal case. J Forensic Med 15:41-42.

Steele RH, Wilhelm DL. 1966. The inflammatory reaction in chemical injury. I. Increased vascular permeability and erythema induced by various chemicals. Br J Exp Pathol 47:612-623.

Stommel P, Muller G, Stucker W et al. 1989. Determination of S-phenylmercapturic acid in the urineAn improvement in the biological monitoring of benzene exposure. Carcinogenesis 10:279-282.

Stover EL, Kincannon DF. 1983. Biological treatability of specific organic compounds found in chemical industry wastewaters. J Water Pollut Control Fed 55:97-109. 
Stubin AI, Brosnan TM, Porter KD, et al. 1996. Organic priority pollutants in New York City municipal wastewaters: 1989-1993. Water Environ Res 68:1037-1044.

Stuermer DH, Ng DJ, Morris CJ. 1982. Organic contaminants in groundwater near an underground coal gasification site in northeastern Wyoming. Environ Sci Technol 16:582-587.

Sturtevant FM. 1952. Studies on the mutagenicity of phenol in Drosophila melanogaster. J Hered 43:217-220.

Subrahmanyam VV, O'Brien PJ. 1985. Phenol oxidation products, formed by a peroxidase reaction, that bind to DNA. Xenobiotica 15:873-885.

Subrahmanyam VV, Doane-Setzer P, Steinmetz KL, et al. 1990a. Phenol-induced stimulation of hydroquinone bioactivation in mouse bone marrow in vivo: Possible implications in benzene myelotoxicity. Toxicology 62:107-116.

*Subrahmanyam VV, Kolachana P, Smith MT. 1990b. Effect of phenol and catechol on the kinetics of human myeloperoxidase-dependent hydroquinone metabolism. Adv Exp Med Biol 283:377-381.

Subrahmanyam VV, Kolachana P, Smith MT. 1991. Hydroxylation of phenol to hydroquinone catalyzed by a human myeloperoxidase-superoxide complex: Possible implications in benzene-induced myelotoxicity. Free Radic Res Commun 15:285-296.

*Subrahmanyam VV, McGirr LG, O'Brien PJ. 1990c. Peroxidase/hydrogen peroxide- or bone marrow homogenate/hydrogen peroxide-mediated activation of phenol and binding to protein. Xenobiotica 20:1369-1378.

Sunesson AL, Gullberg J, Blomquist G. 2001. Airborne chemical compounds on dairy farms. J Environ Monit 3(2):210-216.

Suppiah A, Perry EP. 2005. Jaundice as a presentation of phenol induced hepatotoxocity following injection scherotherapy for haemorrhoids. Surgeon 3(1):43-44.

Suzuki T, Kisara K. 1985. Enhancement of phenol-induced tremor caused by central monoamine depletion. Pharmacol Biochem Behav 22:153-155.

Sze C-C, Shi C-Y, Ong C-N. 1996. Cytotoxicity and DNA strand breaks induced by benzene and its metabolites in Chinese hamster ovary cells. J Appl Toxicol 16:259-264.

Tanaka S, Choe N, Kita T, et al. 1998. Distribution of phenol in a fatal poisoning case determined by gas chromatography/mass spectrometry. J Forensic Sci 43(5):1086-1088.

Tang J, Jin QZ, Shen GH, et al. 1983. Isolation and identification of volatile compounds from fried chicken. J Agric Food Chem 31:1287-1292.

Tesarova E, Packova V. 1983. Gas and high performance liquid chromatography of phenols. Chromatographia 17:269-284.

Thomas K, Colborn T. 1992. Organochlorine endocrine disruptors in human tissue. In: Colborn T, Clement $\mathrm{C}$, eds. Chemically induced alterations in sexual and functional development: The wildlife/human connection. Princeton, NJ: Princeton Scientific Publishing, 365-394. 


\section{REFERENCES}

Thurman C. 1982. Phenol. In: Kirk-Othmer encyclopedia of chemical technology, 3rd ed., Vol 17. New York, NY: John Wiley and Sons, 373-384.

Thurman EM. 1985. Organic geochemistry of natural waters. Boston, MA: Martinus Nijhoff/Dr. W. Junk Publishers, 143-146.

Tomkins BA, Jenkins RA, Griest WH, et al. 1984. Liquid chromatographic determination of phenol and cresols in total particulate matter of cigarette smoke. J Assoc Off Anal Chem 67:919-923.

Tremaine LM, Diamond GL, Quebbemann AJ. 1984. In vivo quantification of renal glucuronide and sulfate conjugation of 1-naphthol and p-nitrophenol in the rat. Biochem Pharmacol 33:419-427.

TRI05. 2007. TRI explorer: Providing access to EPA's toxics release inventory data. Washington, DC: Office of Information Analysis and Access. Office of Environmental Information. U.S. Environmental Protection Agency. Toxics Release Inventory. http://www.epa.gov/triexplorer/. December 22, 2007.

Truppman ES, Ellenby JD. 1979. Major electrocardiographic changes during chemical face peeling. Plast Reconstr Surg 63:44-48.

Tsutsui T, Hayashi N, Maizumi H, et al. 1997. Benzene-, catechol-, hydroquinone- and phenol-induced cell transformation, gene mutations, chromosome aberrations, aneuploidy, sister chromatid exchanges and unscheduled DNA synthesis in Syrian hamster embryo cells. Mutat Res 373(1):113-123.

U.S. Air Force. 1961. Tolerance criteria for continuous inhalation exposure to toxic material. I. Effects on animals of 90-day exposure to phenol, $\mathrm{CCl}_{4}$ and a mixture of indole, skatole, $\mathrm{H}_{2} \mathrm{~S}$ and methyl mercaptan. Wright-Patterson Air Force Base, OH: U.S. Air Force systems command, Aeronautical Systems Division, ASD technical report 61-519(I).

USGS. 1995. Method O-5130-95: Determination of semivolatile organic compounds in bottom sediment by solvent extraction, gel permeation chromatographic fractionation, and capillary-column gas chromatography/mass spectrometry. U.S. Geological Survey. http://pubs.er.usgs.gov/usgspubs/ofr/ofr95719. April 18, 2006.

USGS. 2002. Method O-1433-01: Pesticides and degradates, filtered water, gas chromatography/mass spectrometry. Methods of analysis by the U.S. Geological Survey National Water Quality Laboratory-Determination of wastewater compounds by polystyrene-divinylbenzene solid-phase extraction and capillary-column gas chromatography/mass spectrometry. U.S. Geological Survey.

USITC. 2008. Phenol (hydroxybenzene) and its salts. United States International Trade Commission. http://dataweb.usitc.gov/. July 24, 2008.

Van Duuren BL, Goldschmidt BM. 1976. Cocarcinogenic and tumor promoting agents in tobacco carcinogenesis. J Natl Cancer Inst 56:1237-1242.

Van Duuren BL, Blazej T, Goldschmidt BM, et al. 1971. Cocarcinogenesis studies on mouse skin and inhibition of tumor induction. J Natl Cancer Inst 46:1039-1044.

Van Duuren BL, Katz C, Goldschmidt BM. 1973. Cocarcinogenic agents in tobacco carcinogenesis. J Natl Cancer Inst 51:703-705. 


\section{REFERENCES}

Van Roosmalen PB, Purdham J, Drummond I. 1981. An improved method for the determination of phenol in the urine of workers exposed to benzene or phenol. Int Arch Occup Environ Health 48:159163.

Van Rossum P, Webb RG. 1978. Isolation of organic water pollutants by XAD resins and carbon. J Chromatogr 150:381-392.

Vernot EH, MacEwen JD, Haun CC, et al. 1977. Acute toxicity and skin corrosion data for some organic and inorganic compounds and aqueous solutions. Toxicol Appl Pharmacol 42:417-424.

Viccellio P. 1998. Phenols. In: Emergency toxicology. 2nd ed. Philadelphia, PA: Lippincott-Raven Publishers, 355-362.

Vieira I, Sonnier M, Cresteil T. 1996. Developmental expression of CYP2E1 in the human liver: Hypermethylation control of gene expression during the neonatal period. Eur J Biochem 238:476-483.

von Oettingen WF, Sharpless NE. 1946. The toxicity and toxic manifestations of 2,2-bis(pchlorophenyl)-1,1,1-trichloroethane (DDT) as influenced by chemical changes in the molecule. J Pharmacol Exp Ther 88:400-413.

Wallace J. 1996. Phenol. In: Kroschwitz JI, Howe-Grant M, eds. Kirk-Othmer encyclopedia of chemical toxicology. $4^{\text {th }}$ ed. New York, NY: John Wiley \& Sons, 592-602.

Wallace JC, Basu I, Hites RA. 1996. Sampling and analysis artifacts caused by elevated indoor air polychlorinated biphenyl concentrations. Environ Sci Technol 30:2730-2734.

*Wallin H, Morgenstern R. 1990. Activation of microsomal glutathione transferase activity by reactive intermediates formed during the metabolism of phenol. Chem-Biol Interact 75:185-199.

Warner MA, Harper JV. 1985. Cardiac dysrhythmias associated with chemical peeling with phenol. Anesthesiology 62:366-367.

Waxman DJ, Dannan GA, Guengerich FP. 1985. Regulation of rat hepatic cytochrome P-450: Agedependent expression, hormonal imprinting, and xenobiotic inducibility of sex-specific isoenzymes. Biochemistry 24:4409-4417.

Weitering JG, Kriggsheld KR, Mulder GJ. 1979. The availability of inorganic sulfate as a rate limiting factor in the sulfate conjugation of xenobiotics in the rat. Biochem Pharmacol 28:757-762.

West JR, Smith HW, Chasis H. 1948. Glomerular filtration rate, effective renal blood flow, and maximal tubular excretory capacity in infancy. J Pediatr 32a:10-18.

Wexler MR, Halon DA, Teitelbaum A, et al. 1984. The prevention of cardiac arrhythmias produced in an animal model by the topical application of a phenol preparation in common use for face peeling. Plast Reconstr Surg 73:595-598.

Wheldrake JF, Baudinette RV, Hewitt S. 1978. The metabolism of phenol in desert rodent Notomys alexis. Comp Biochem Physiol 61C:103-107.

WHO. 1994. Phenol. Environmental health criteria 161. Geneva, Switzerland: United Nations Environment Programme. International Labour Organisation. World Health Organization. 
WHO. 2000. Air quality guidelines. 2nd ed. Geneva, Switzerland: World Health Organization. http://www.euro.who.int/air/activities/20050223_4. March 08, 2006.

WHO. 2004. Guidelines for drinking water qualities. 3rd ed. Geneva, Switzerland: World Health Organization. http://www.who.int/water_sanitation_health/dwq/gdwq3/en/. March 08, 2006.

Widdowson EM, Dickerson JWT. 1964. Chemical composition of the body. In: Comar CL, Bronner F, eds. Mineral metabolism: An advanced treatise, volume II, The elements part A. New York, NY: Academic Press.

Wilcosky TC, Tyroler HA. 1983. Mortality from heart disease among workers exposed to solvents. J Occup Med 25:879-885.

Wood K. 1978. The use of phenol as a neurolytic agent: A review. Pain 5:205-229.

Wynder E, Hoffmann D. 1961. A study of tobacco carcinogenesis. VIII. The role of acidic fractions as promoters. Cancer 14:1306-1315.

Xing B, McGill WB, Dudas MJ. 1994. Sorption of phenol by selected polymers: Isotherms, energetics, and polarity. Environ Sci Technol 28:466-473.

York RG. 1997. Oral (gavage) developmental toxicity study of phenol in rats. Proctor \& Gamble Company. Submitted to the U.S. Environmental Protection Agency under TSCA Section 8D.

OTS0573686.

Young DR, Gossett RW, Baird RB, et al. 1983. Wastewater inputs and marine bioaccumulation of priority pollutants organics off southern California. In: Water chlorination: Environmental impacts and health effects. 4:871-884.

Yrjanheikki E. 1978. A new method of personnel sampling and analyzing of phenol. Am Ind Hyg Assoc J 39:326-330.

Zamponi GW, Ing D, French RJ. 1994. Arrhythmias during phenol therapies: A specific action on cardiac sodium channels? Circulation 89:914.

Zhao F, Mayura K, Hutchinson RW, et al. 1995. Developmental toxicity and structure-activity relationships of chlorophenols using human embryonic palatal mesenchymal cells. Toxicol Lett 78:3542.

Zhu J, Newhook R, Marro L, et al. 2005. Selected volatile organic compounds in residential air in the city of Ottawa, Canada. Environ Sci Technol 39(11):3964-3971.

Ziegler EE, Edwards BB, Jensen RL, et al. 1978. Absorption and retention of lead by infants. Pediatr Res 12:29-34. 


\section{GLOSSARY}

Absorption-The taking up of liquids by solids, or of gases by solids or liquids.

Acute Exposure-Exposure to a chemical for a duration of 14 days or less, as specified in the Toxicological Profiles.

Adsorption-The adhesion in an extremely thin layer of molecules (as of gases, solutes, or liquids) to the surfaces of solid bodies or liquids with which they are in contact.

Adsorption Coefficient $\left(\mathbf{K}_{\mathbf{o c}}\right)$ - The ratio of the amount of a chemical adsorbed per unit weight of organic carbon in the soil or sediment to the concentration of the chemical in solution at equilibrium.

Adsorption Ratio (Kd) - The amount of a chemical adsorbed by sediment or soil (i.e., the solid phase) divided by the amount of chemical in the solution phase, which is in equilibrium with the solid phase, at a fixed solid/solution ratio. It is generally expressed in micrograms of chemical sorbed per gram of soil or sediment.

Benchmark Dose (BMD) - Usually defined as the lower confidence limit on the dose that produces a specified magnitude of changes in a specified adverse response. For example, a BMD 10 would be the dose at the $95 \%$ lower confidence limit on a $10 \%$ response, and the benchmark response (BMR) would be $10 \%$. The BMD is determined by modeling the dose response curve in the region of the dose response relationship where biologically observable data are feasible.

Benchmark Dose Model—A statistical dose-response model applied to either experimental toxicological or epidemiological data to calculate a BMD.

Bioconcentration Factor (BCF) - The quotient of the concentration of a chemical in aquatic organisms at a specific time or during a discrete time period of exposure divided by the concentration in the surrounding water at the same time or during the same period.

Biomarkers-Broadly defined as indicators signaling events in biologic systems or samples. They have been classified as markers of exposure, markers of effect, and markers of susceptibility.

Cancer Effect Level (CEL) - The lowest dose of chemical in a study, or group of studies, that produces significant increases in the incidence of cancer (or tumors) between the exposed population and its appropriate control.

Carcinogen-A chemical capable of inducing cancer.

Case-Control Study - A type of epidemiological study that examines the relationship between a particular outcome (disease or condition) and a variety of potential causative agents (such as toxic chemicals). In a case-controlled study, a group of people with a specified and well-defined outcome is identified and compared to a similar group of people without outcome.

Case Report-Describes a single individual with a particular disease or exposure. These may suggest some potential topics for scientific research, but are not actual research studies.

Case Series-Describes the experience of a small number of individuals with the same disease or exposure. These may suggest potential topics for scientific research, but are not actual research studies. 
Ceiling Value-A concentration of a substance that should not be exceeded, even instantaneously.

Chronic Exposure-Exposure to a chemical for 365 days or more, as specified in the Toxicological Profiles.

Cohort Study-A type of epidemiological study of a specific group or groups of people who have had a common insult (e.g., exposure to an agent suspected of causing disease or a common disease) and are followed forward from exposure to outcome. At least one exposed group is compared to one unexposed group.

Cross-sectional Study-A type of epidemiological study of a group or groups of people that examines the relationship between exposure and outcome to a chemical or to chemicals at one point in time.

Data Needs-Substance-specific informational needs that if met would reduce the uncertainties of human health assessment.

Developmental Toxicity-The occurrence of adverse effects on the developing organism that may result from exposure to a chemical prior to conception (either parent), during prenatal development, or postnatally to the time of sexual maturation. Adverse developmental effects may be detected at any point in the life span of the organism.

Dose-Response Relationship-The quantitative relationship between the amount of exposure to a toxicant and the incidence of the adverse effects.

Embryotoxicity and Fetotoxicity-Any toxic effect on the conceptus as a result of prenatal exposure to a chemical; the distinguishing feature between the two terms is the stage of development during which the insult occurs. The terms, as used here, include malformations and variations, altered growth, and in utero death.

Environmental Protection Agency (EPA) Health Advisory-An estimate of acceptable drinking water levels for a chemical substance based on health effects information. A health advisory is not a legally enforceable federal standard, but serves as technical guidance to assist federal, state, and local officials.

Epidemiology-Refers to the investigation of factors that determine the frequency and distribution of disease or other health-related conditions within a defined human population during a specified period.

Genotoxicity-A specific adverse effect on the genome of living cells that, upon the duplication of affected cells, can be expressed as a mutagenic, clastogenic, or carcinogenic event because of specific alteration of the molecular structure of the genome.

Half-life-A measure of rate for the time required to eliminate one half of a quantity of a chemical from the body or environmental media.

Immediately Dangerous to Life or Health (IDLH) - The maximum environmental concentration of a contaminant from which one could escape within 30 minutes without any escape-impairing symptoms or irreversible health effects.

Immunologic Toxicity-The occurrence of adverse effects on the immune system that may result from exposure to environmental agents such as chemicals. 
Immunological Effects-Functional changes in the immune response.

Incidence-The ratio of individuals in a population who develop a specified condition to the total number of individuals in that population who could have developed that condition in a specified time period.

Intermediate Exposure-Exposure to a chemical for a duration of 15-364 days, as specified in the Toxicological Profiles.

In Vitro-Isolated from the living organism and artificially maintained, as in a test tube.

In Vivo-Occurring within the living organism.

Lethal Concentration $(\mathrm{LO})\left(\mathbf{L C}_{\mathrm{LO}}\right)$ - The lowest concentration of a chemical in air that has been reported to have caused death in humans or animals.

Lethal Concentration ${ }_{(50)}\left(\mathbf{L C}_{50}\right)$ - A calculated concentration of a chemical in air to which exposure for a specific length of time is expected to cause death in $50 \%$ of a defined experimental animal population.

Lethal Dose $\mathbf{L}_{(\mathbf{L O})}\left(\mathbf{L D}_{\mathbf{L 0}}\right)$ - The lowest dose of a chemical introduced by a route other than inhalation that has been reported to have caused death in humans or animals.

Lethal Dose $_{(50)}\left(\mathbf{L D}_{50}\right)$ - The dose of a chemical that has been calculated to cause death in $50 \%$ of a defined experimental animal population.

Lethal Time $_{(50)}\left(\mathbf{L T}_{\mathbf{5 0}}\right)$ - A calculated period of time within which a specific concentration of a chemical is expected to cause death in $50 \%$ of a defined experimental animal population.

Lowest-Observed-Adverse-Effect Level (LOAEL) - The lowest exposure level of chemical in a study, or group of studies, that produces statistically or biologically significant increases in frequency or severity of adverse effects between the exposed population and its appropriate control.

Lymphoreticular Effects-Represent morphological effects involving lymphatic tissues such as the lymph nodes, spleen, and thymus.

Malformations-Permanent structural changes that may adversely affect survival, development, or function.

Minimal Risk Level (MRL) — An estimate of daily human exposure to a hazardous substance that is likely to be without an appreciable risk of adverse noncancer health effects over a specified route and duration of exposure.

Modifying Factor (MF)—A value (greater than zero) that is applied to the derivation of a Minimal Risk Level (MRL) to reflect additional concerns about the database that are not covered by the uncertainty factors. The default value for a MF is 1 .

Morbidity-State of being diseased; morbidity rate is the incidence or prevalence of disease in a specific population.

Mortality-Death; mortality rate is a measure of the number of deaths in a population during a specified interval of time. 
Mutagen-A substance that causes mutations. A mutation is a change in the DNA sequence of a cell's DNA. Mutations can lead to birth defects, miscarriages, or cancer.

Necropsy - The gross examination of the organs and tissues of a dead body to determine the cause of death or pathological conditions.

Neurotoxicity-The occurrence of adverse effects on the nervous system following exposure to a chemical.

No-Observed-Adverse-Effect Level (NOAEL) - The dose of a chemical at which there were no statistically or biologically significant increases in frequency or severity of adverse effects seen between the exposed population and its appropriate control. Effects may be produced at this dose, but they are not considered to be adverse.

Octanol-Water Partition Coefficient $\left(\mathbf{K}_{\mathbf{o w}}\right)$ - The equilibrium ratio of the concentrations of a chemical in $n$-octanol and water, in dilute solution.

Odds Ratio (OR) - A means of measuring the association between an exposure (such as toxic substances and a disease or condition) that represents the best estimate of relative risk (risk as a ratio of the incidence among subjects exposed to a particular risk factor divided by the incidence among subjects who were not exposed to the risk factor). An OR of greater than 1 is considered to indicate greater risk of disease in the exposed group compared to the unexposed group.

Organophosphate or Organophosphorus Compound-A phosphorus-containing organic compound and especially a pesticide that acts by inhibiting cholinesterase.

Permissible Exposure Limit (PEL) — An Occupational Safety and Health Administration (OSHA) allowable exposure level in workplace air averaged over an 8-hour shift of a 40-hour workweek.

Pesticide-General classification of chemicals specifically developed and produced for use in the control of agricultural and public health pests.

Pharmacokinetics - The dynamic behavior of a material in the body, used to predict the fate (disposition) of an exogenous substance in an organism. Utilizing computational techniques, it provides the means of studying the absorption, distribution, metabolism, and excretion of chemicals by the body.

Pharmacokinetic Model-A set of equations that can be used to describe the time course of a parent chemical or metabolite in an animal system. There are two types of pharmacokinetic models: data-based and physiologically-based. A data-based model divides the animal system into a series of compartments, which, in general, do not represent real, identifiable anatomic regions of the body, whereas the physiologically-based model compartments represent real anatomic regions of the body.

Physiologically Based Pharmacodynamic (PBPD) Model-A type of physiologically based doseresponse model that quantitatively describes the relationship between target tissue dose and toxic end points. These models advance the importance of physiologically based models in that they clearly describe the biological effect (response) produced by the system following exposure to an exogenous substance.

Physiologically Based Pharmacokinetic (PBPK) Model-Comprised of a series of compartments representing organs or tissue groups with realistic weights and blood flows. These models require a 
variety of physiological information: tissue volumes, blood flow rates to tissues, cardiac output, alveolar ventilation rates, and possibly membrane permeabilities. The models also utilize biochemical information, such as air/blood partition coefficients, and metabolic parameters. PBPK models are also called biologically based tissue dosimetry models.

Prevalence-The number of cases of a disease or condition in a population at one point in time.

Prospective Study-A type of cohort study in which the pertinent observations are made on events occurring after the start of the study. A group is followed over time.

$\mathbf{q}_{1}{ }^{*}$-The upper-bound estimate of the low-dose slope of the dose-response curve as determined by the multistage procedure. The $\mathrm{q}_{1} *$ can be used to calculate an estimate of carcinogenic potency, the incremental excess cancer risk per unit of exposure (usually $\mu \mathrm{g} / \mathrm{L}$ for water, $\mathrm{mg} / \mathrm{kg} / \mathrm{day}$ for food, and $\mu \mathrm{g} / \mathrm{m}^{3}$ for air).

Recommended Exposure Limit (REL) - A National Institute for Occupational Safety and Health (NIOSH) time-weighted average (TWA) concentration for up to a 10-hour workday during a 40-hour workweek.

Reference Concentration (RfC) — An estimate (with uncertainty spanning perhaps an order of magnitude) of a continuous inhalation exposure to the human population (including sensitive subgroups) that is likely to be without an appreciable risk of deleterious noncancer health effects during a lifetime. The inhalation reference concentration is for continuous inhalation exposures and is appropriately expressed in units of $\mathrm{mg} / \mathrm{m}^{3}$ or $\mathrm{ppm}$.

Reference Dose (RfD) - An estimate (with uncertainty spanning perhaps an order of magnitude) of the daily exposure of the human population to a potential hazard that is likely to be without risk of deleterious effects during a lifetime. The RfD is operationally derived from the no-observed-adverse-effect level (NOAEL, from animal and human studies) by a consistent application of uncertainty factors that reflect various types of data used to estimate RfDs and an additional modifying factor, which is based on a professional judgment of the entire database on the chemical. The RfDs are not applicable to nonthreshold effects such as cancer.

Reportable Quantity (RQ) - The quantity of a hazardous substance that is considered reportable under the Comprehensive Environmental Response, Compensation, and Liability Act (CERCLA). Reportable quantities are (1) 1 pound or greater or (2) for selected substances, an amount established by regulation either under CERCLA or under Section 311 of the Clean Water Act. Quantities are measured over a 24-hour period.

Reproductive Toxicity-The occurrence of adverse effects on the reproductive system that may result from exposure to a chemical. The toxicity may be directed to the reproductive organs and/or the related endocrine system. The manifestation of such toxicity may be noted as alterations in sexual behavior, fertility, pregnancy outcomes, or modifications in other functions that are dependent on the integrity of this system.

Retrospective Study-A type of cohort study based on a group of persons known to have been exposed at some time in the past. Data are collected from routinely recorded events, up to the time the study is undertaken. Retrospective studies are limited to causal factors that can be ascertained from existing records and/or examining survivors of the cohort.

Risk-The possibility or chance that some adverse effect will result from a given exposure to a chemical. 
Risk Factor-An aspect of personal behavior or lifestyle, an environmental exposure, or an inborn or inherited characteristic that is associated with an increased occurrence of disease or other health-related event or condition.

Risk Ratio-The ratio of the risk among persons with specific risk factors compared to the risk among persons without risk factors. A risk ratio greater than 1 indicates greater risk of disease in the exposed group compared to the unexposed group.

Short-Term Exposure Limit (STEL) - The American Conference of Governmental Industrial Hygienists (ACGIH) maximum concentration to which workers can be exposed for up to 15 minutes continually. No more than four excursions are allowed per day, and there must be at least 60 minutes between exposure periods. The daily Threshold Limit Value-Time Weighted Average (TLV-TWA) may not be exceeded.

Standardized Mortality Ratio (SMR) - A ratio of the observed number of deaths and the expected number of deaths in a specific standard population.

Target Organ Toxicity-This term covers a broad range of adverse effects on target organs or physiological systems (e.g., renal, cardiovascular) extending from those arising through a single limited exposure to those assumed over a lifetime of exposure to a chemical.

Teratogen-A chemical that causes structural defects that affect the development of an organism.

Threshold Limit Value (TLV)—An American Conference of Governmental Industrial Hygienists (ACGIH) concentration of a substance to which most workers can be exposed without adverse effect. The TLV may be expressed as a Time Weighted Average (TWA), as a Short-Term Exposure Limit (STEL), or as a ceiling limit (CL).

Time-Weighted Average (TWA) - An allowable exposure concentration averaged over a normal 8-hour workday or 40-hour workweek.

Toxic Dose $\mathbf{( 5 0}_{(\mathbf{5 0}}\left(\mathbf{T D}_{\mathbf{5 0}}\right)$ - A calculated dose of a chemical, introduced by a route other than inhalation, which is expected to cause a specific toxic effect in $50 \%$ of a defined experimental animal population.

Toxicokinetic-The absorption, distribution, and elimination of toxic compounds in the living organism.

Uncertainty Factor (UF) — A factor used in operationally deriving the Minimal Risk Level (MRL) or Reference Dose (RfD) or Reference Concentration (RfC) from experimental data. UFs are intended to account for (1) the variation in sensitivity among the members of the human population, (2) the uncertainty in extrapolating animal data to the case of human, (3) the uncertainty in extrapolating from data obtained in a study that is of less than lifetime exposure, and (4) the uncertainty in using lowestobserved-adverse-effect level (LOAEL) data rather than no-observed-adverse-effect level (NOAEL) data. A default for each individual UF is 10; if complete certainty in data exists, a value of 1 can be used; however, a reduced UF of 3 may be used on a case-by-case basis, 3 being the approximate logarithmic average of 10 and 1 .

Xenobiotic-Any chemical that is foreign to the biological system. 


\section{APPENDIX A. ATSDR MINIMAL RISK LEVELS AND WORKSHEETS}

The Comprehensive Environmental Response, Compensation, and Liability Act (CERCLA) [42 U.S.C. 9601 et seq.], as amended by the Superfund Amendments and Reauthorization Act (SARA) [Pub. L. 99499], requires that the Agency for Toxic Substances and Disease Registry (ATSDR) develop jointly with the U.S. Environmental Protection Agency (EPA), in order of priority, a list of hazardous substances most commonly found at facilities on the CERCLA National Priorities List (NPL); prepare toxicological profiles for each substance included on the priority list of hazardous substances; and assure the initiation of a research program to fill identified data needs associated with the substances.

The toxicological profiles include an examination, summary, and interpretation of available toxicological information and epidemiologic evaluations of a hazardous substance. During the development of toxicological profiles, Minimal Risk Levels (MRLs) are derived when reliable and sufficient data exist to identify the target organ(s) of effect or the most sensitive health effect(s) for a specific duration for a given route of exposure. An MRL is an estimate of the daily human exposure to a hazardous substance that is likely to be without appreciable risk of adverse noncancer health effects over a specified duration of exposure. MRLs are based on noncancer health effects only and are not based on a consideration of cancer effects. These substance-specific estimates, which are intended to serve as screening levels, are used by ATSDR health assessors to identify contaminants and potential health effects that may be of concern at hazardous waste sites. It is important to note that MRLs are not intended to define clean-up or action levels.

MRLs are derived for hazardous substances using the no-observed-adverse-effect level/uncertainty factor approach. They are below levels that might cause adverse health effects in the people most sensitive to such chemical-induced effects. MRLs are derived for acute (1-14 days), intermediate (15-364 days), and chronic (365 days and longer) durations and for the oral and inhalation routes of exposure. Currently, MRLs for the dermal route of exposure are not derived because ATSDR has not yet identified a method suitable for this route of exposure. MRLs are generally based on the most sensitive chemical-induced end point considered to be of relevance to humans. Serious health effects (such as irreparable damage to the liver or kidneys, or birth defects) are not used as a basis for establishing MRLs. Exposure to a level above the MRL does not mean that adverse health effects will occur.

MRLs are intended only to serve as a screening tool to help public health professionals decide where to look more closely. They may also be viewed as a mechanism to identify those hazardous waste sites that 
are not expected to cause adverse health effects. Most MRLs contain a degree of uncertainty because of the lack of precise toxicological information on the people who might be most sensitive (e.g., infants, elderly, nutritionally or immunologically compromised) to the effects of hazardous substances. ATSDR uses a conservative (i.e., protective) approach to address this uncertainty consistent with the public health principle of prevention. Although human data are preferred, MRLs often must be based on animal studies because relevant human studies are lacking. In the absence of evidence to the contrary, ATSDR assumes that humans are more sensitive to the effects of hazardous substance than animals and that certain persons may be particularly sensitive. Thus, the resulting MRL may be as much as 100 -fold below levels that have been shown to be nontoxic in laboratory animals.

Proposed MRLs undergo a rigorous review process: Health Effects/MRL Workgroup reviews within the Division of Toxicology and Environmental Medicine, expert panel peer reviews, and agency-wide MRL Workgroup reviews, with participation from other federal agencies and comments from the public. They are subject to change as new information becomes available concomitant with updating the toxicological profiles. Thus, MRLs in the most recent toxicological profiles supersede previously published levels. For additional information regarding MRLs, please contact the Division of Toxicology and Environmental Medicine, Agency for Toxic Substances and Disease Registry, 1600 Clifton Road NE, Mailstop F-32, Atlanta, Georgia 30333. 


\section{MINIMAL RISK LEVEL (MRL) WORKSHEET}

$\begin{array}{ll}\text { Chemical Name: } & \text { Phenol } \\ \text { CAS Numbers: } & 108-95-2 \\ \text { Date: } & \text { July 2008 } \\ \text { Profile Status: } & \text { Draft 3 Post-Public } \\ \text { Route: } & \text { [] Inhalation [x] Oral } \\ \text { Duration: } & \text { [x] Acute [] Intermediate [] Chronic } \\ \text { Graph Key: } & 13 \\ \text { Species: } & \text { Rat }\end{array}$

Minimal Risk Level: 1 [x] mg/kg/day [ ] ppm

Reference: York. 1997. Oral (gavage) developmental toxicity study of phenol in rats. Procter \& Gamble Co. Submitted to the U.S. Environmental Protection Agency under TSCA Section 8D. OTS0573686.

Experimental design: Groups of pregnant Sprague-Dawley rats (25/dose group) were dosed 3 times daily with $0,20,40$, or $120 \mathrm{mg}$ phenol $/ \mathrm{kg}$ (total daily doses of $0,60,120$, and $360 \mathrm{mg} / \mathrm{kg}$ ) by gavage in water on gestation days (GDs) 6-15; the dosing volume was $10 \mathrm{~mL} / \mathrm{kg}$. Maternal end points evaluated included clinical signs, body weight, and food consumption. Dams were also observed for abortions and premature deliveries. Dams were sacrificed on GD 20 and a gross necropsy was conducted. The uterus was examined for pregnancy, number and distribution of implantations, live and dead fetuses, and early and late resorptions. Fetuses were weighed and examined for sex and gross external alterations. Half of the fetuses were examined for soft tissue alterations and the remaining fetuses were examined for skeletal alterations.

Effect noted in study and corresponding doses: One dam in the $360 \mathrm{mg} / \mathrm{kg} /$ day group died on GD 11 and the death was attributed to phenol treatment. Clinical signs considered treatment-related included excess salivation and tachypnea in rats exposed to $360 \mathrm{mg} / \mathrm{kg} /$ day. Gross necropsy of the dams did not reveal any treatment-related alterations. In the $120 \mathrm{mg} / \mathrm{kg} /$ day group, maternal body weight gain was significantly reduced for GDs 6-16 (11\%) and for GDs 12-16 (19\%), whereas in the $360 \mathrm{mg} / \mathrm{kg} / \mathrm{day}$ group, body weight gain was reduced $38 \%$ for gestation days $6-16$. Maternal final body weight in the $360 \mathrm{mg} / \mathrm{kg} /$ day group was reduced, but $<10 \%$ relative to controls. Food consumption was reduced in the $360 \mathrm{mg} / \mathrm{kg} /$ day group by $16 \%$ for GDs $6-20$ and by $15 \%$ for GDs $0-20$; in the $120 \mathrm{mg} / \mathrm{kg} / \mathrm{day}$ group, food consumption for GDs 6-16 was reduced $11 \%$. Fetal body weight at the $360 \mathrm{mg} / \mathrm{kg} /$ day level was reduced $5-7 \%$ relative to controls. There was a significant decrease in ossification sites on the hindlimb metatarsals in the $360 \mathrm{mg} / \mathrm{kg} /$ day group. At the 120 and $360 \mathrm{mg} / \mathrm{kg} /$ day dose levels, there were increases in litters with fetuses with "any alteration" and with "any variation", but neither reached statistical significance and there were no clear dose-response relationships. There were no significant effects on corpora lutea, implantations, litter sizes, live fetuses, early and late resorptions, and percent resorbed conceptuses. Based on decreased fetal body weight and delayed ossification, the dose of $360 \mathrm{mg} / \mathrm{kg} / \mathrm{day}$ is a LOAEL for developmental effects; the NOAEL is $120 \mathrm{mg} / \mathrm{kg} /$ day. Based on decreased weight gain during gestation, the dose of $120 \mathrm{mg} / \mathrm{kg} /$ day is a LOAEL for decreased maternal body weight gain; the NOAEL is $60 \mathrm{mg} / \mathrm{kg} /$ day.

Data from York (1997) were analyzed using the BMD approach for MRL derivation. BMD models in the EPA Benchmark Dose Software (BMDS version 2.0) (linear, polynomial, power, and Hill models) were fit to the maternal body weight gain data to determine potential points of departure for the MRL. The linear model with homogeneous variance, which is the simplest model and the model that provided the best fit for the data, was selected. In the absence of a clear criteria as to what level of change in weight 
gain during pregnancy should be considered adverse, the benchmark response (BMR) was defined as a change in mean body weight gain equal to one standard deviation from the control mean (EPA 2000c). The corresponding BMD was $152 \mathrm{mg} / \mathrm{kg} / \mathrm{day}$; the corresponding benchmark dose limit (BMDL) was $125 \mathrm{mg} / \mathrm{kg} /$ day. Applying an uncertainty factor of 100 (10 for animal to human extrapolation and 10 for human variability) to the BMDL results in an acute-duration oral MRL of $1 \mathrm{mg} / \mathrm{kg} / \mathrm{day}$.

Dose and end point used for MRL derivation: BMDL of $125 \mathrm{mg} / \mathrm{kg} /$ day for decreased maternal weight gain.

\section{[ ] NOAEL [ ] LOAEL [x] BMDL}

Uncertainty Factors used in MRL derivation:

[x] 10 for extrapolation from animals to humans

[x] 10 for human variability

Was a conversion factor used from ppm in food or water to a $\mathrm{mg} /$ body weight dose? Not applicable.

If an inhalation study in animals, list conversion factors used in determining human equivalent dose: Not applicable.

$\underline{\text { Was a conversion used from intermittent to continuous exposure? }}$ Not applicable.

Other additional studies or pertinent information that lend support to this MRL: In another developmental study, rats were gavaged with phenol in doses of $0,30,60$, and $120 \mathrm{mg} / \mathrm{kg} /$ day in a dosing volume of $5 \mathrm{~mL} / \mathrm{kg}$ during GDs 6-15 (NTP 1983a). There was no maternal toxicity, but mean fetal body weight at this dose level was approximately 7\% lower than controls. However, since historical control data showed that the concurrent control fetal weight for the CD rat was much higher $(22 \%)$ than the historical control weight and a larger litter size in the high-dose group may have contributed to the smaller fetal weight in the high-dose group, the dose of $120 \mathrm{mg} / \mathrm{kg} /$ day can be considered an equivocal LOAEL for developmental effects; the NOAEL was $60 \mathrm{mg} / \mathrm{kg} /$ day and supports the NOAEL of $60 \mathrm{mg} / \mathrm{kg} /$ day identified in the York (1997) study.

As discussed in Section 2.3, effects of phenol administered to animals by oral gavage are different than those observed in drinking water studies. Phenol administered by gavage is much more toxic than administered in the drinking water and this is related to the pharmacokinetics of phenol. Furthermore, it has been shown that the volume of administration is important; the smaller the volume, the greater the toxicity of a given amount of phenol (NTP 1983a). Studies have shown that the toxicity of phenol is correlated with peak blood concentration rather than with total dose, such as the area under the phenol blood concentration curve (AUC) (Hiser et al. 1994). In general, NOAELs in oral gavage studies were 510 times lower than in drinking water studies. The York (1997) study was considered an appropriate study for MRL derivation because it used a divided dosing protocol that resembles more closely a potential environmental exposure scenario to phenol. 


\section{BENCHMARK MODELING OF MATERNAL WEIGHT GAIN IN RATS}

Benchmark dose models in the EPA Benchmark Dose Software (BMDS version 2.0) (linear, polynomial, power, and Hill models) were fit to the maternal body weight gain data (see Tables A-1, and A-2, and Figure A-1) to determine potential points of departure for the MRL. The linear model with homogeneous variance (which was identical to the power model), was selected because it was the simplest model and provided the best fit. In the absence of a clear criteria as to what level of change in weight gain during pregnancy should be considered adverse, the BMR was defined as a change in mean body weight gain equal to one standard deviation from the control mean (EPA 2000c).

\section{Table A-1. Data for the Change in Body Weight Gain in Pregnant Rats Exposed to Phenol on Gestation Days 6-15}

\begin{tabular}{cllc}
\hline Dose $(\mathrm{mg} / \mathrm{kg} /$ day $)$ & Number of animals tested & Body weight gain $(\mathrm{g})$ & Standard deviation \\
\hline 0 & 23 & 64 & 10.7 \\
60 & 25 & 58 & 9.4 \\
120 & 23 & 56.8 & 10.8 \\
360 & 25 & 39.8 & 9.5 \\
\hline
\end{tabular}

Source: York 1997

The corresponding BMD was $152.1 \mathrm{mg} / \mathrm{kg} /$ day; the corresponding BMDL was $124.6 \mathrm{mg} / \mathrm{kg} / \mathrm{day}$. Applying an uncertainty factor of 100 (10 for animal to human extrapolation and 10 for human variability) to the BMDL results in an acute-duration oral MRL of $1 \mathrm{mg} / \mathrm{kg} / \mathrm{day}$.

\section{Table A-2. Model Predictions for Changes in Body Weight Gain in Pregnant Rats Exposed to Phenol on Gestation Days 6-15}

\begin{tabular}{lllll}
\hline Model & $\mathrm{BMD}_{1 \text { stddev }}(\mathrm{mg} / \mathrm{kg} /$ day $)$ & $\mathrm{BMDL}_{1 \text { stddev }}(\mathrm{mg} / \mathrm{kg} /$ day $)$ & $\mathrm{p}$-value & AlC-fitted $^{\mathrm{a}}$ \\
\hline Linear $^{\mathrm{b}, \mathrm{c}}$ & 152.1 & 124.6 & 0.6055 & 542.79 \\
2-degree polynomial $^{\mathrm{c}}$ & 157.0 & 124.6 & 0.3191 & 544.78 \\
Power $^{\mathrm{b}}$ & 152.1 & 124.6 & 0.6055 & 542.79 \\
Hill & 151.2 & 123.7 & 0.3156 & 544.79 \\
\hline
\end{tabular}

${ }^{\mathrm{a} V}$ Values $<0.05$ fail to meet conventional goodness-of-fit criteria.

${ }^{\mathrm{b}}$ Best-fitting models

${ }^{\mathrm{c}}$ The polynomial coefficients are restricted to be negative.

AIC = Akaike's Information Criteria; $B M D=$ benchmark dose; $B M D L=$ lower confidence limit (95\%) on the benchmark dose; NA = not applicable; $p=p$ value from the Chi-squared test

Source: York 1997 
Figure A-1. Changes in Body Weight Gain in Pregnant Rats Exposed to Phenol on Gestation Days 6-15*

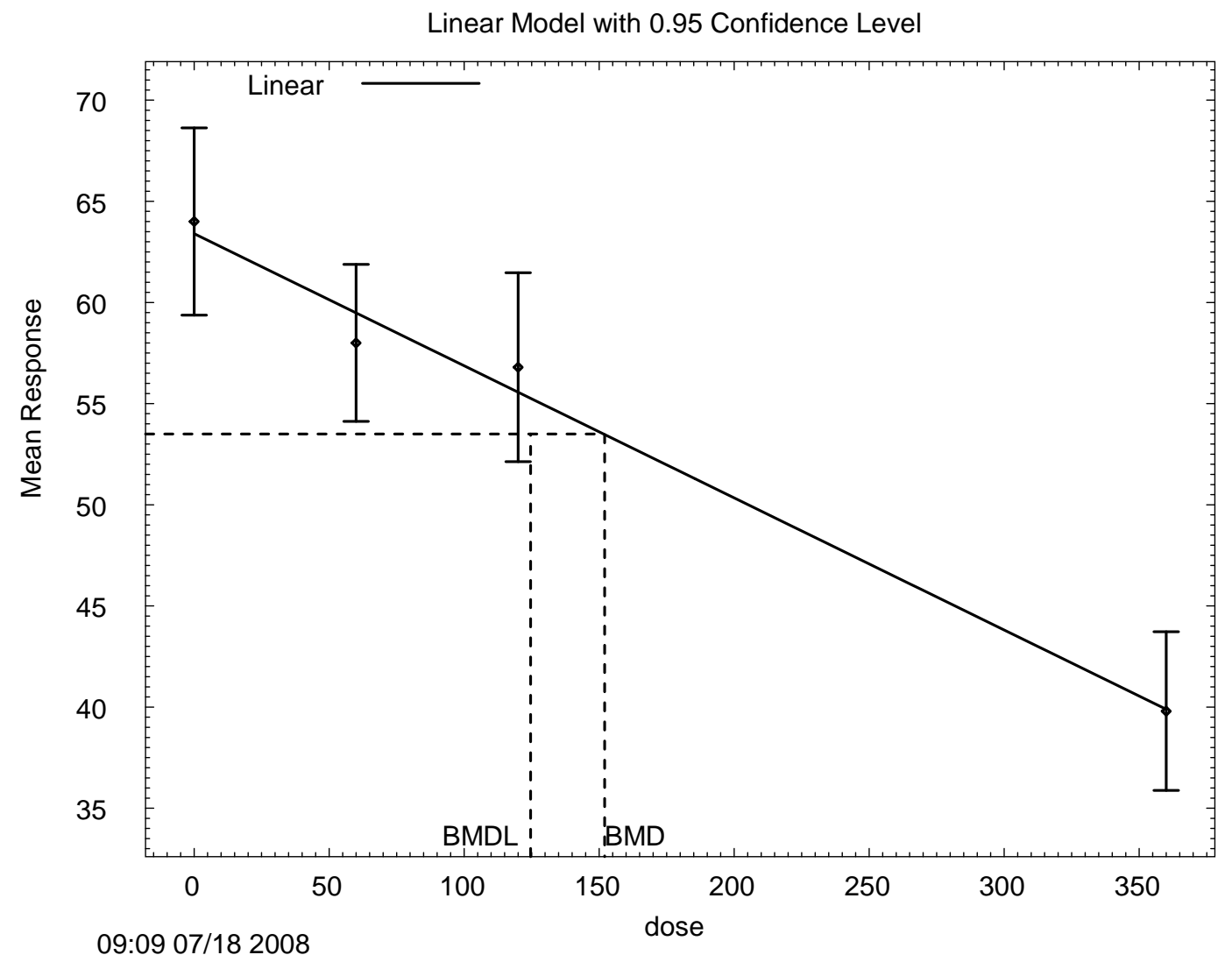

${ }^{\star}$ BMDs and BMDLs indicated are for a change of 1 standard deviation and are in units of $\mathrm{mg} / \mathrm{kg} / \mathrm{day}$.

Source: York 1997

The homogeneous variance linear model form of the response function for the change in maternal body weight gain is:

$\mathrm{Y}[\mathrm{dose}]=$ beta_0 + beta_1 $*$ dose + beta_2*dose^ $2+\ldots$

Linear Model Parameter Estimates for the Change in Maternal Body Weight Gain:

Variable

alpha

beta_0

beta_1
Estimate

98.6

63.4

$-0.07$
Standard Error

14.2

1.4

0.007 


\section{APPENDIX B. USER'S GUIDE}

\section{Chapter 1}

\section{Public Health Statement}

This chapter of the profile is a health effects summary written in non-technical language. Its intended audience is the general public, especially people living in the vicinity of a hazardous waste site or chemical release. If the Public Health Statement were removed from the rest of the document, it would still communicate to the lay public essential information about the chemical.

The major headings in the Public Health Statement are useful to find specific topics of concern. The topics are written in a question and answer format. The answer to each question includes a sentence that will direct the reader to chapters in the profile that will provide more information on the given topic.

\section{Chapter 2}

\section{Relevance to Public Health}

This chapter provides a health effects summary based on evaluations of existing toxicologic, epidemiologic, and toxicokinetic information. This summary is designed to present interpretive, weightof-evidence discussions for human health end points by addressing the following questions:

1. What effects are known to occur in humans?

2. What effects observed in animals are likely to be of concern to humans?

3. What exposure conditions are likely to be of concern to humans, especially around hazardous waste sites?

The chapter covers end points in the same order that they appear within the Discussion of Health Effects by Route of Exposure section, by route (inhalation, oral, and dermal) and within route by effect. Human data are presented first, then animal data. Both are organized by duration (acute, intermediate, chronic). In vitro data and data from parenteral routes (intramuscular, intravenous, subcutaneous, etc.) are also considered in this chapter.

The carcinogenic potential of the profiled substance is qualitatively evaluated, when appropriate, using existing toxicokinetic, genotoxic, and carcinogenic data. ATSDR does not currently assess cancer potency or perform cancer risk assessments. Minimal Risk Levels (MRLs) for noncancer end points (if derived) and the end points from which they were derived are indicated and discussed.

Limitations to existing scientific literature that prevent a satisfactory evaluation of the relevance to public health are identified in the Chapter 3 Data Needs section.

\section{Interpretation of Minimal Risk Levels}

Where sufficient toxicologic information is available, ATSDR has derived MRLs for inhalation and oral routes of entry at each duration of exposure (acute, intermediate, and chronic). These MRLs are not meant to support regulatory action, but to acquaint health professionals with exposure levels at which adverse health effects are not expected to occur in humans. 
MRLs should help physicians and public health officials determine the safety of a community living near a chemical emission, given the concentration of a contaminant in air or the estimated daily dose in water. MRLs are based largely on toxicological studies in animals and on reports of human occupational exposure.

MRL users should be familiar with the toxicologic information on which the number is based. Chapter 2, "Relevance to Public Health," contains basic information known about the substance. Other sections such as Chapter 3 Section 3.9, "Interactions with Other Substances," and Section 3.10, "Populations that are Unusually Susceptible" provide important supplemental information.

MRL users should also understand the MRL derivation methodology. MRLs are derived using a modified version of the risk assessment methodology that the Environmental Protection Agency (EPA) provides (Barnes and Dourson 1988) to determine reference doses (RfDs) for lifetime exposure.

To derive an MRL, ATSDR generally selects the most sensitive end point which, in its best judgement, represents the most sensitive human health effect for a given exposure route and duration. ATSDR cannot make this judgement or derive an MRL unless information (quantitative or qualitative) is available for all potential systemic, neurological, and developmental effects. If this information and reliable quantitative data on the chosen end point are available, ATSDR derives an MRL using the most sensitive species (when information from multiple species is available) with the highest no-observed-adverse-effect level (NOAEL) that does not exceed any adverse effect levels. When a NOAEL is not available, a lowest-observed-adverse-effect level (LOAEL) can be used to derive an MRL, and an uncertainty factor (UF) of 10 must be employed. Additional uncertainty factors of 10 must be used both for human variability to protect sensitive subpopulations (people who are most susceptible to the health effects caused by the substance) and for interspecies variability (extrapolation from animals to humans). In deriving an MRL, these individual uncertainty factors are multiplied together. The product is then divided into the inhalation concentration or oral dosage selected from the study. Uncertainty factors used in developing a substance-specific MRL are provided in the footnotes of the levels of significant exposure (LSE) tables.

\section{Chapter 3}

\section{Health Effects}

\section{Tables and Figures for Levels of Significant Exposure (LSE)}

Tables and figures are used to summarize health effects and illustrate graphically levels of exposure associated with those effects. These levels cover health effects observed at increasing dose concentrations and durations, differences in response by species, MRLs to humans for noncancer end points, and EPA's estimated range associated with an upper- bound individual lifetime cancer risk of 1 in 10,000 to 1 in $10,000,000$. Use the LSE tables and figures for a quick review of the health effects and to locate data for a specific exposure scenario. The LSE tables and figures should always be used in conjunction with the text. All entries in these tables and figures represent studies that provide reliable, quantitative estimates of NOAELs, LOAELs, or Cancer Effect Levels (CELs).

The legends presented below demonstrate the application of these tables and figures. Representative examples of LSE Table 3-1 and Figure 3-1 are shown. The numbers in the left column of the legends correspond to the numbers in the example table and figure. 


\section{LEGEND}

\section{See Sample LSE Table 3-1 (page B-6)}

(1) Route of Exposure. One of the first considerations when reviewing the toxicity of a substance using these tables and figures should be the relevant and appropriate route of exposure. Typically when sufficient data exist, three LSE tables and two LSE figures are presented in the document. The three LSE tables present data on the three principal routes of exposure, i.e., inhalation, oral, and dermal (LSE Tables 3-1, 3-2, and 3-3, respectively). LSE figures are limited to the inhalation (LSE Figure 3-1) and oral (LSE Figure 3-2) routes. Not all substances will have data on each route of exposure and will not, therefore, have all five of the tables and figures.

(2) Exposure Period. Three exposure periods - acute (less than 15 days), intermediate (15364 days), and chronic (365 days or more) - are presented within each relevant route of exposure. In this example, an inhalation study of intermediate exposure duration is reported. For quick reference to health effects occurring from a known length of exposure, locate the applicable exposure period within the LSE table and figure.

(3) Health Effect. The major categories of health effects included in LSE tables and figures are death, systemic, immunological, neurological, developmental, reproductive, and cancer. NOAELs and LOAELs can be reported in the tables and figures for all effects but cancer. Systemic effects are further defined in the "System" column of the LSE table (see key number $18)$.

(4) Key to Figure. Each key number in the LSE table links study information to one or more data points using the same key number in the corresponding LSE figure. In this example, the study represented by key number 18 has been used to derive a NOAEL and a Less Serious LOAEL (also see the two "18r" data points in sample Figure 3-1).

(5) Species. The test species, whether animal or human, are identified in this column. Chapter 2, "Relevance to Public Health," covers the relevance of animal data to human toxicity and Section 3.4, "Toxicokinetics," contains any available information on comparative toxicokinetics. Although NOAELs and LOAELs are species specific, the levels are extrapolated to equivalent human doses to derive an MRL.

(6) Exposure Frequency/Duration. The duration of the study and the weekly and daily exposure regimens are provided in this column. This permits comparison of NOAELs and LOAELs from different studies. In this case (key number 18), rats were exposed to "Chemical x" via inhalation for 6 hours/day, 5 days/week, for 13 weeks. For a more complete review of the dosing regimen, refer to the appropriate sections of the text or the original reference paper (i.e., Nitschke et al. 1981).

(7) System. This column further defines the systemic effects. These systems include respiratory, cardiovascular, gastrointestinal, hematological, musculoskeletal, hepatic, renal, and dermal/ocular. "Other" refers to any systemic effect (e.g., a decrease in body weight) not covered in these systems. In the example of key number 18, one systemic effect (respiratory) was investigated.

(8) NOAEL. A NOAEL is the highest exposure level at which no harmful effects were seen in the organ system studied. Key number 18 reports a NOAEL of $3 \mathrm{ppm}$ for the respiratory system, which was used to derive an intermediate exposure, inhalation MRL of $0.005 \mathrm{ppm}$ (see footnote "b"). 
(9) LOAEL. A LOAEL is the lowest dose used in the study that caused a harmful health effect. LOAELs have been classified into "Less Serious" and "Serious" effects. These distinctions help readers identify the levels of exposure at which adverse health effects first appear and the gradation of effects with increasing dose. A brief description of the specific end point used to quantify the adverse effect accompanies the LOAEL. The respiratory effect reported in key number 18 (hyperplasia) is a Less Serious LOAEL of $10 \mathrm{ppm}$. MRLs are not derived from Serious LOAELs.

(10) Reference. The complete reference citation is given in Chapter 9 of the profile.

(11) CEL. A CEL is the lowest exposure level associated with the onset of carcinogenesis in experimental or epidemiologic studies. CELs are always considered serious effects. The LSE tables and figures do not contain NOAELs for cancer, but the text may report doses not causing measurable cancer increases.

(12) Footnotes. Explanations of abbreviations or reference notes for data in the LSE tables are found in the footnotes. Footnote "b" indicates that the NOAEL of 3 ppm in key number 18 was used to derive an MRL of $0.005 \mathrm{ppm}$.

\section{LEGEND}

\section{See Sample Figure 3-1 (page B-7)}

LSE figures graphically illustrate the data presented in the corresponding LSE tables. Figures help the reader quickly compare health effects according to exposure concentrations for particular exposure periods.

(13) Exposure Period. The same exposure periods appear as in the LSE table. In this example, health effects observed within the acute and intermediate exposure periods are illustrated.

(14) Health Effect. These are the categories of health effects for which reliable quantitative data exists. The same health effects appear in the LSE table.

(15) Levels of Exposure. Concentrations or doses for each health effect in the LSE tables are graphically displayed in the LSE figures. Exposure concentration or dose is measured on the log scale "y" axis. Inhalation exposure is reported in $\mathrm{mg} / \mathrm{m}^{3}$ or $\mathrm{ppm}$ and oral exposure is reported in $\mathrm{mg} / \mathrm{kg} /$ day.

(16) NOAEL. In this example, the open circle designated $18 \mathrm{r}$ identifies a NOAEL critical end point in the rat upon which an intermediate inhalation exposure MRL is based. The key number 18 corresponds to the entry in the LSE table. The dashed descending arrow indicates the extrapolation from the exposure level of $3 \mathrm{ppm}$ (see entry 18 in the table) to the MRL of $0.005 \mathrm{ppm}$ (see footnote "b" in the LSE table).

(17) CEL. Key number $38 \mathrm{~m}$ is one of three studies for which CELs were derived. The diamond symbol refers to a CEL for the test species-mouse. The number 38 corresponds to the entry in the LSE table. 
(18) Estimated Upper-Bound Human Cancer Risk Levels. This is the range associated with the upperbound for lifetime cancer risk of 1 in 10,000 to 1 in 10,000,000. These risk levels are derived from the EPA's Human Health Assessment Group's upper-bound estimates of the slope of the cancer dose response curve at low dose levels $\left(\mathrm{q}_{1}{ }^{*}\right)$.

(19) Key to LSE Figure. The Key explains the abbreviations and symbols used in the figure. 


\section{SAMPLE}

Table 3-1. Levels of Significant Exposure to [Chemical x] - Inhalation

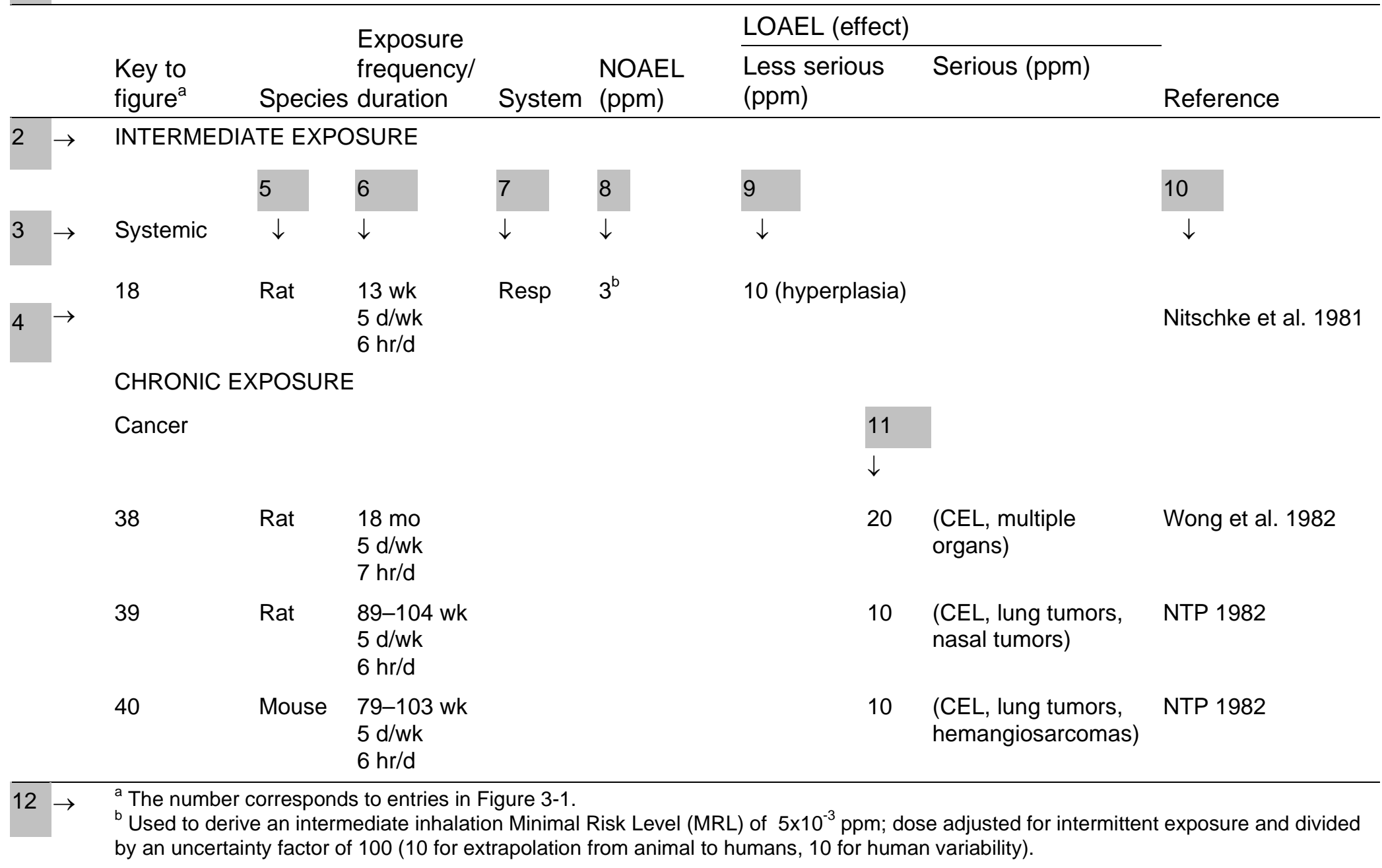




\section{SAMPLE}

13

Figure 3-1. Levels of Significant Exposure to [Chemical X] - Inhalation
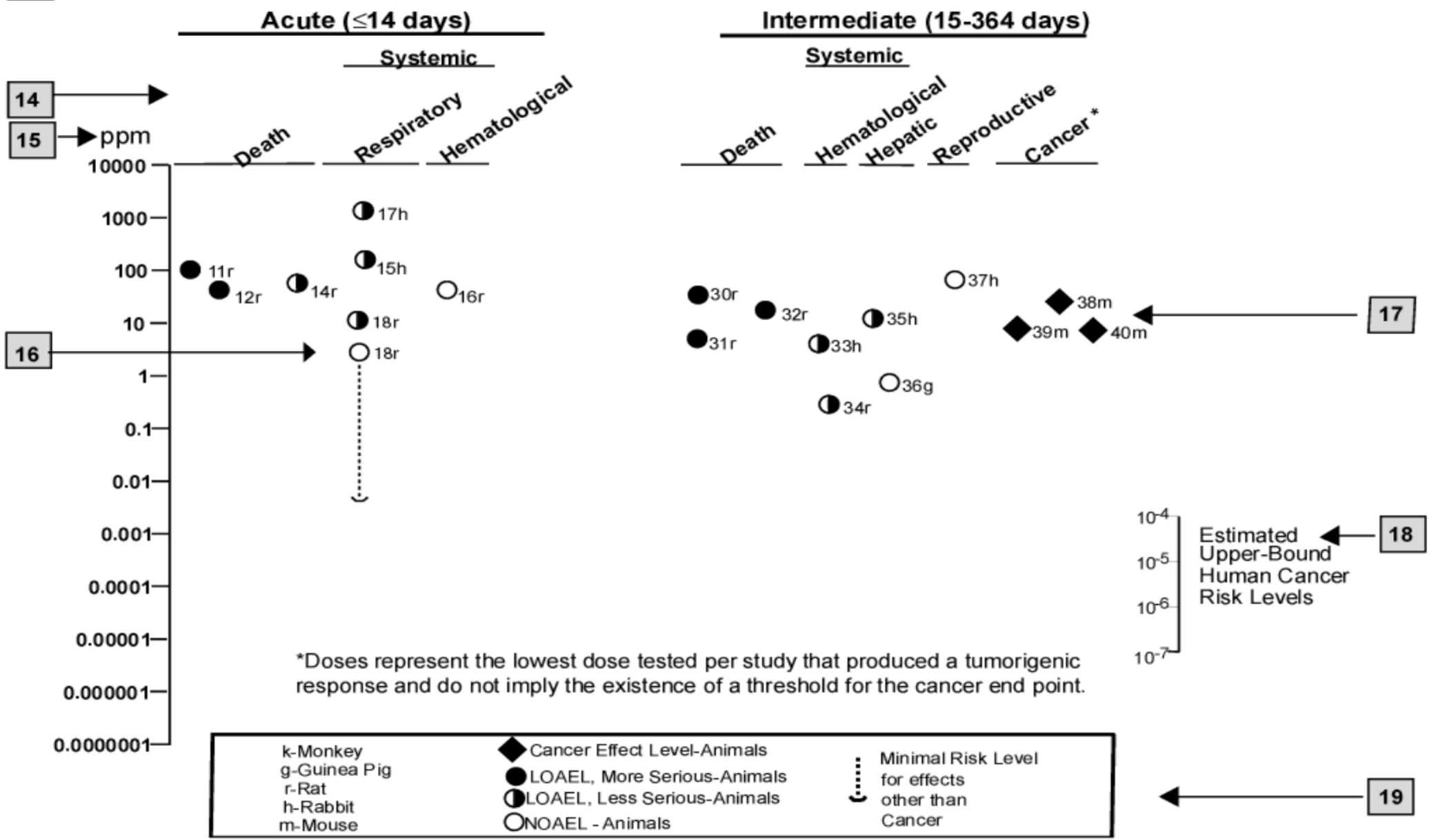
APPENDIX B

This page is intentionally blank. 


\section{APPENDIX C. ACRONYMS, ABBREVIATIONS, AND SYMBOLS}

\begin{tabular}{|c|c|}
\hline ACGIH & American Conference of Governmental Industrial Hygienists \\
\hline ACOEM & American College of Occupational and Environmental Medicine \\
\hline ADI & acceptable daily intake \\
\hline ADME & absorption, distribution, metabolism, and excretion \\
\hline AED & atomic emission detection \\
\hline AFID & alkali flame ionization detector \\
\hline AFOSH & Air Force Office of Safety and Health \\
\hline ALT & alanine aminotransferase \\
\hline AML & acute myeloid leukemia \\
\hline $\mathrm{AOAC}$ & Association of Official Analytical Chemists \\
\hline AOEC & Association of Occupational and Environmental Clinics \\
\hline AP & alkaline phosphatase \\
\hline APHA & American Public Health Association \\
\hline AST & aspartate aminotransferase \\
\hline atm & atmosphere \\
\hline ATSDR & Agency for Toxic Substances and Disease Registry \\
\hline AWQC & Ambient Water Quality Criteria \\
\hline BAT & best available technology \\
\hline $\mathrm{BCF}$ & bioconcentration factor \\
\hline BEI & Biological Exposure Index \\
\hline BMD & benchmark dose \\
\hline BMR & benchmark response \\
\hline $\mathrm{BSC}$ & Board of Scientific Counselors \\
\hline $\mathrm{C}$ & centigrade \\
\hline CAA & Clean Air Act \\
\hline CAG & Cancer Assessment Group of the U.S. Environmental Protection Agency \\
\hline CAS & Chemical Abstract Services \\
\hline $\mathrm{CDC}$ & Centers for Disease Control and Prevention \\
\hline CEL & cancer effect level \\
\hline CELDS & Computer-Environmental Legislative Data System \\
\hline CERCLA & Comprehensive Environmental Response, Compensation, and Liability Act \\
\hline CFR & Code of Federal Regulations \\
\hline $\mathrm{Ci}$ & curie \\
\hline $\mathrm{CI}$ & confidence interval \\
\hline $\mathrm{CL}$ & ceiling limit value \\
\hline CLP & Contract Laboratory Program \\
\hline $\mathrm{cm}$ & centimeter \\
\hline CML & chronic myeloid leukemia \\
\hline CPSC & Consumer Products Safety Commission \\
\hline CWA & Clean Water Act \\
\hline DHEW & Department of Health, Education, and Welfare \\
\hline DHHS & Department of Health and Human Services \\
\hline DNA & deoxyribonucleic acid \\
\hline DOD & Department of Defense \\
\hline DOE & Department of Energy \\
\hline DOL & Department of Labor \\
\hline DOT & Department of Transportation \\
\hline $\mathrm{DOT} / \mathrm{UN} /$ & Department of Transportation/United Nations/ \\
\hline NA/IMCO & North America/Intergovernmental Maritime Dangerous Goods Code \\
\hline
\end{tabular}




\begin{tabular}{|c|c|}
\hline DWEL & drinking water exposure level \\
\hline ECD & electron capture detection \\
\hline ECG/EKG & electrocardiogram \\
\hline EEG & electroencephalogram \\
\hline EEGL & Emergency Exposure Guidance Level \\
\hline EPA & Environmental Protection Agency \\
\hline $\mathrm{F}$ & Fahrenheit \\
\hline $\mathrm{F}_{1}$ & first-filial generation \\
\hline FAO & Food and Agricultural Organization of the United Nations \\
\hline FDA & Food and Drug Administration \\
\hline FEMA & Federal Emergency Management Agency \\
\hline FIFRA & Federal Insecticide, Fungicide, and Rodenticide Act \\
\hline FPD & flame photometric detection \\
\hline fpm & feet per minute \\
\hline FR & Federal Register \\
\hline FSH & follicle stimulating hormone \\
\hline $\mathrm{g}$ & gram \\
\hline GC & gas chromatography \\
\hline gd & gestational day \\
\hline GLC & gas liquid chromatography \\
\hline GPC & gel permeation chromatography \\
\hline HPLC & high-performance liquid chromatography \\
\hline HRGC & high resolution gas chromatography \\
\hline HSDB & Hazardous Substance Data Bank \\
\hline IARC & International Agency for Research on Cancer \\
\hline IDLH & immediately dangerous to life and health \\
\hline ILO & International Labor Organization \\
\hline IRIS & Integrated Risk Information System \\
\hline $\mathrm{Kd}$ & adsorption ratio \\
\hline $\mathrm{kg}$ & kilogram \\
\hline $\mathrm{kkg}$ & metric ton \\
\hline $\mathrm{K}_{\mathrm{oc}}$ & organic carbon partition coefficient \\
\hline $\mathrm{K}_{\mathrm{ow}}$ & octanol-water partition coefficient \\
\hline $\mathrm{L}$ & liter \\
\hline $\mathrm{LC}$ & liquid chromatography \\
\hline $\mathrm{LC}_{50}$ & lethal concentration, $50 \%$ kill \\
\hline $\mathrm{LC}_{\mathrm{Lo}}$ & lethal concentration, low \\
\hline $\mathrm{LD}_{50}$ & lethal dose, $50 \%$ kill \\
\hline $\mathrm{LD}_{\mathrm{Lo}}$ & lethal dose, low \\
\hline LDH & lactic dehydrogenase \\
\hline LH & luteinizing hormone \\
\hline LOAEL & lowest-observed-adverse-effect level \\
\hline LSE & Levels of Significant Exposure \\
\hline $\mathrm{LT}_{50}$ & lethal time, $50 \%$ kill \\
\hline $\mathrm{m}$ & meter \\
\hline MA & trans,trans-muconic acid \\
\hline MAL & maximum allowable level \\
\hline $\mathrm{mCi}$ & millicurie \\
\hline MCL & maximum contaminant level \\
\hline MCLG & maximum contaminant level goal \\
\hline MF & modifying factor \\
\hline
\end{tabular}




\begin{tabular}{|c|c|}
\hline MFO & mixed function oxidase \\
\hline $\mathrm{mg}$ & milligram \\
\hline $\mathrm{mL}$ & milliliter \\
\hline $\mathrm{mm}$ & millimeter \\
\hline $\mathrm{mmHg}$ & millimeters of mercury \\
\hline mmol & millimole \\
\hline mppcf & millions of particles per cubic foot \\
\hline MRL & Minimal Risk Level \\
\hline MS & mass spectrometry \\
\hline NAAQS & National Ambient Air Quality Standard \\
\hline NAS & National Academy of Science \\
\hline NATICH & National Air Toxics Information Clearinghouse \\
\hline NATO & North Atlantic Treaty Organization \\
\hline NCE & normochromatic erythrocytes \\
\hline NCEH & National Center for Environmental Health \\
\hline NCI & National Cancer Institute \\
\hline ND & not detected \\
\hline NFPA & National Fire Protection Association \\
\hline ng & nanogram \\
\hline NHANES & National Health and Nutrition Examination Survey \\
\hline NIEHS & National Institute of Environmental Health Sciences \\
\hline $\mathrm{NIOSH}$ & National Institute for Occupational Safety and Health \\
\hline NIOSHTIC & NIOSH's Computerized Information Retrieval System \\
\hline NLM & National Library of Medicine \\
\hline $\mathrm{nm}$ & nanometer \\
\hline nmol & nanomole \\
\hline NOAEL & no-observed-adverse-effect level \\
\hline NOES & National Occupational Exposure Survey \\
\hline NOHS & National Occupational Hazard Survey \\
\hline NPD & nitrogen phosphorus detection \\
\hline NPDES & National Pollutant Discharge Elimination System \\
\hline NPL & National Priorities List \\
\hline NR & not reported \\
\hline NRC & National Research Council \\
\hline NS & not specified \\
\hline NSPS & New Source Performance Standards \\
\hline NTIS & National Technical Information Service \\
\hline NTP & National Toxicology Program \\
\hline ODW & Office of Drinking Water, EPA \\
\hline OERR & Office of Emergency and Remedial Response, EPA \\
\hline OHM/TADS & Oil and Hazardous Materials/Technical Assistance Data System \\
\hline OPP & Office of Pesticide Programs, EPA \\
\hline OPPT & Office of Pollution Prevention and Toxics, EPA \\
\hline OPPTS & Office of Prevention, Pesticides and Toxic Substances, EPA \\
\hline OR & odds ratio \\
\hline OSHA & Occupational Safety and Health Administration \\
\hline OSW & Office of Solid Waste, EPA \\
\hline OTS & Office of Toxic Substances \\
\hline OW & Office of Water \\
\hline OWRS & Office of Water Regulations and Standards, EPA \\
\hline PAH & polycyclic aromatic hydrocarbon \\
\hline
\end{tabular}




$\begin{array}{ll}\text { PBPD } & \text { physiologically based pharmacodynamic } \\ \text { PBPK } & \text { physiologically based pharmacokinetic } \\ \text { PCE } & \text { polychromatic erythrocytes } \\ \text { PEL } & \text { permissible exposure limit } \\ \text { pg } & \text { picogram } \\ \text { PHS } & \text { Public Health Service } \\ \text { PID } & \text { photo ionization detector } \\ \text { pmol } & \text { picomole } \\ \text { PMR } & \text { proportionate mortality ratio } \\ \text { ppb } & \text { parts per billion } \\ \text { ppm } & \text { parts per million } \\ \text { ppt } & \text { parts per trillion } \\ \text { PSNS } & \text { pretreatment standards for new sources } \\ \text { RBC } & \text { red blood cell } \\ \text { REL } & \text { recommended exposure level/limit } \\ \text { RfC } & \text { reference concentration } \\ \text { RfD } & \text { reference dose } \\ \text { RNA } & \text { ribonucleic acid } \\ \text { RQ } & \text { reportable quantity } \\ \text { RTECS } & \text { Registry of Toxic Effects of Chemical Substances } \\ \text { SARA } & \text { Superfund Amendments and Reauthorization Act } \\ \text { SCE } & \text { sister chromatid exchange } \\ \text { SGOT } & \text { serum glutamic oxaloacetic transaminase } \\ \text { SGPT } & \text { serum glutamic pyruvic transaminase } \\ \text { SIC } & \text { standard industrial classification } \\ \text { SIM } & \text { selected ion monitoring } \\ \text { SMCL } & \text { secondary maximum contaminant level } \\ \text { SMR } & \text { standardized mortality ratio } \\ \text { SNARL } & \text { suggested no adverse response level } \\ \text { SPEGL } & \text { Short-Term Public Emergency Guidance Level } \\ \text { STEL } & \text { short term exposure limit } \\ \text { STORET } & \text { Storage and Retrieval } \\ \text { TD } & \text { toxic dose, 50\% specific toxic effect } \\ \text { TLV } & \text { threshold limit value } \\ \text { TOC } & \text { total organic carbon } \\ \text { TPQ } & \text { threshold planning quantity } \\ \text { TRI } & \text { Toxics Release Inventory } \\ \text { TSCA } & \text { Toxic Substances Control Act } \\ \text { TWA } & \text { time-weighted average } \\ \text { UF } & \text { uncertainty factor } \\ \text { U.S. } & \text { United States } \\ \text { USDA } & \text { United States Department of Agriculture } \\ \text { USGS } & \text { United States Geological Survey } \\ \text { VOC } & \text { volatile organic compound } \\ \text { WBC } & \text { white blood cell } \\ \text { WHO } & \text { World Health Organization } \\ & \end{array}$




$\begin{array}{ll}> & \text { greater than } \\ \geq & \text { greater than or equal to } \\ = & \text { equal to } \\ < & \text { less than } \\ \leq & \text { less than or equal to } \\ \% & \text { percent } \\ \alpha & \text { alpha } \\ \beta & \text { beta } \\ \gamma & \text { gamma } \\ \delta & \text { delta } \\ \mu \mathrm{m} & \text { micrometer } \\ \mu \mathrm{g} & \text { microgram } \\ \mathrm{q}^{*} & \text { cancer slope factor } \\ - & \text { negative } \\ + & \text { positive } \\ (+) & \text { weakly positive result } \\ (-) & \text { weakly negative result }\end{array}$


APPENDIX C

This page is intentionally blank. 


\section{APPENDIX D. INDEX}

absorbed dose 122

acetylcholine 124

adrenal gland $14,67,75,131$

adsorption. $156,170,176$

aerobic $9,157,158,159$

alanine aminotransferase (see ALT) 10,36

ALT (see alanine aminotransferase)

$10,36,37,65,82,131$

ambient air

$149,159,164,165,170$

anaerobic.....

$9,158,159$

aspartate aminotransferase (see AST) 35,36

AST (see aspartate aminotransferase) $10,36,37,65,82,131$

bioaccumulation 156,170

bioavailability 170

bioconcentration factor 156

biodegradation

biomarker $10,149,155,156,158$

blood cell count $12,36,64$

body weight effects $39,68,85$

breast milk. $5,10,137,166$

cancer $4,22,44,75,86,87,118,121,185$ carcinogen. $86,185,188$ carcinogenic $14,21,75,86,111$ carcinogenicity $106,111,121,122,123,124,134,135,172,173,181$ carcinoma 86,87 cardiac arrhythmia 6, 129 cardiovascular cardiovascular effects 134, 136 catechol (112, chromosomal aberrations $87,91,92$

clearance 95,100

consumer products $1,9,164,165,166,167,171$

death $4,11,18,21,22,23,35,43,44,61,70,75,76,77,85,120,125,127,188$ deoxyribonucleic acid (see DNA) $87,88,90$ dermal effects $38,39,67,83$

disinfectant $1,5,67,70,119,143$

DNA (see deoxyribonucleic acid) $87,88,89,90,91,92,93,99,114,122$

dopamine. 20,71

elimination half-time. .99

endocrine. $67,115,116$

endocrine effects 67

erythema $11,63,77,83,125$

estrogen receptor 117

estrogenic 117

fetal tissue .98

fetus. .117

gastrointestinal effects general population. genotoxic. genotoxicity.

$34,35,63,81$ $10,22,35,44,122,134,165,188$ $21,91,112,132$ $87,129,132$ 
germinal cell. glucuronide $100,101,103,104,114,121,136,137,173$

groundwater $100,101,103,104,114,121,136,137,173$

growth retardation $2,9,149,155,156,158,160,161,163,164,171$

half-life $9,94,95,107,122,149,155,157,158$ hematological effects $35,64,81$ hematopoietic $36,65,82,124$ hepatic effects $43,173,174,176$

hydrolysis $111,112,115,124$ hydroquinone $149,155,157$ hydroxyl radical immune system 40 immunological immunological effects. $12,13,19,21,35,40,41,69,70,85,129,130,131$

$\mathrm{K}_{\text {ow }}$ $19,69,130$

$\mathrm{LD}_{50}$ 141

leukemia $45,61,76,77,99$

lymphoreticular. $14,75,131$ metabolic effects $13,20,41,69,70,85$

micronuclei .40

milk $5,121,137$

musculoskeletal effects $36,65,81,82$ neurobehavioral 116 neurochemical $12,19,130$ norepinephrine 20,71

nuclear. 82,114 ocular effects $68,84,130$

odds ratio..... .44

pharmacodynamic 108 pharmacokinetic placenta $108,109,110,118,121$

placental barrier 121,137

rate constant 94,157

renal effects $38,66,82$

retention $93,94,95,157$

salivation 18,85

solubility 155

spermatogonia 91

sulfate $93,97,101,103,104,113,115,120,121,124,135,136,137,173,179,180$ thyroid 67,99 toxicokinetic $12,16,21,127,135$ tremors tumors volatility $77,86,87,124$ volatilization 158 155,170 
